\title{
Geostrophic Vortex Dynamics
}

by

\author{
Lorenzo M. Polvani \\ B.Sc. (1981) McGill University \\ M.Sc. (1982) McGill University \\ SUBMITTED IN PARTIAL FULFILLMENT \\ OF THE REQUIREMENTS FOR THE DEGREE \\ OF DOCTOR OF PHILOSOPHY \\ at the \\ MASSACHUSETTS INSTITUTE OF TECHNOLOGY \\ and the \\ WOODS HOLE OCEANOGRAPHIC INSTITUTION
}

10 August, 1988

(C) Lorenzo M. Polvani, 1988

The author hereby grants MIT and WHOI the permission to reproduce and distribute copies of this thesis in whole or in part.

Signature of the Author M.I.T.W.H.O.I. Joint Program in Physical Oçeano faphy

Certified by

Glenn R. Flier

Thesis Co-Supepisor

(Nфman J) Zabusky

Mesis Co-Supenisor

Accepted by

CarlWunsch

Chairman, Joint Committee for Physical Oceanography 


\section{Abstract}

By generalizing the method of contour dynamics to the quasigeostrophic two layer model, we have proposed and solved a number of fundamental problems in the dynamics of rotating and stratified vorticity fields. A variety of rotating and translating potential vorticity equilibria ( $V$-states) in one and two layers have been obtained, shedding new light on potential vorticity dynamics in the geostrophic context. In particular, the equivalent barotropic model is shown to be a singular limit of the two-layer model for scales large compared to the radius of deformation.

The question of coalescence of two vortices in the same layer (merger) and in different layers (alignment) is studied in detail. Critical initial separation distances for coalescence are numerically established as functions of the radius of deformation and the relative thickness of the layers at rest. The connection between coalescence and the existence of stable rotating doubly-connected Vstates is shown to be an illuminating generalization of the Euler results.

The question of filamentation of two-dimensional vorticity interfaces is addressed from a new geometrical perspective. The analysis of the topology of the streamfunction in a frame of reference rotating with the instantaneous angular velocity of the vorticity distribution (the corotating frame) is shown to yield new powerful insights on the nonlinear evolution of the vorticity field. In particular, the presence of hyperbolic (critical) points of the corotating streamfunction that come in contact with the vorticity interface is found to be directly responsible for the generation of filaments.

The importance of the position of the critical points of the comoving streamfunction is found to generalize to the two-layer quasigeostrophic context. They are shown to play the crucial role in determining the limits, in parameter space, on the existence of a number of two-layer rotating and translating potential vorticity equilibria. 
बू

से 


\section{Acknowledgments}

Quo quisquam optimus sit, pessimus, si hoc ipsum, quod optimus sit, adscribat sibit

\section{St. Bernard of Clairvaux}

The composition of a doctoral dissertation is an accomplishment of such magnitude that few authors can truthfully claim to be entirely creditable for its completion. In the present case, it is truly a great pleasure, as well as an honor, for me to acknowledge here the contributions of the many people who have made this thesis possible.

Among the many teachers I have had the opportunity to learn from over the past few years, three deserve to be mentioned in particular: Dr. Joe Pedlosky must be credited for teaching me how to "fly with my own wings", Dr. Glenn Flierl for teaching me most of the GFD I know and Dr. Norman Zabusky for teaching me how to think in a "nonlinear" way.

I must also acknowledge the contribution of Dr. Roberta Young, who introduced me to the mysteries of Internet Protocols, Cray JCL and many other abstruse matters dealing with computers. Dr. Steve Meacham has been a valuable help over the past two years, and I wish to thank him for many enlightening conversations and for his patience in answering my innumerable questions.

My fellow students at MIT and in the Joint Program have provided the friendly atmosphere which has allowed me to endure the difficulties of graduate student life. Among them, I wish to mention in particular Mr. Joe Chang, who shared my office for five whole years without complaining once that my music was too loud, Ms. Luanne Thompson, for her maternal support, which included bringing me lemon squares when I had to spend long weekends at work to complete this manuscript, and one friend, who asked to remain anonymous, whose charm and wit made the completion of this work more bearable.

I must acknowledge the generosity of Dr. Bob Weller and Ms. Nancy Pennington who allowed me to use their facilities when, according to the best predictions of Murphy's Law, my Macintosh suffered a hardware failure two weeks before this thesis was due.

Many other friends and colleagues would undoubtedly deserve to be mentioned here, even if their names do not explicitly appear. I wish them to know, however, that their support has been invaluable, and that they should consider this thesis to be as much theirs as it is mine.

Last, and not only not léast, but most certainly greatest, has been the moral support of my family, who encouraged me from the beginning and who have never ceased to be for me a source of strength and inspiration.

† A man becomes worst from being best, if he ascribes his best to his own efforts. 


\section{Table of Contents}

Abstract

Acknowledgments

Table of Contents

1. Prolegomena $\quad 8$

1.1. The Two-Layer Model 9

1.2. A concise review of Euler results 12

1.3. Contour Dynamics 18

1.4. Synopsis 22

1.5. Algorithms 24

2. Two-Layer Dynamics with $\Pi_{2}=0$

2.1. Introduction $\quad 40$

2.2. Upper-layer V-states 44

2.3. Merger of Vorticity in the Same Layer 50

2.4. Doubly-Connected Rotating One-Layer V-states 56

3. Two-Layer Dynamics with G.P.V. in both Layers 79

3.1. The Alignment Problem in the 2-Layer Model 80

3.2. Doubly-Connected Rotating Two-Layer V-States 85

3.3. Two-Layer Dipoles 94 
4. Critical Points and Filamentation

4.1. The Linearly Perturbed Rankine Vortex 139

4.2. The Filamentation of Kirchhoff Ellipses 144

4.3. Critical Points in the Equivalent Barotropic Model $(\gamma>>1)$

5. Conclusion

6. Addenda 176

A. Generalized Kirchhoff Vortices 176

B. The 2-Level Rankine Vortex: Critical Points \& Stability 181

C. Tripolar Coherent Euler Vortices , 186

7. Appendices 201

A. High Order Schemes for Moment Evaluation 201

B. Explicit Formuals for V-State Algorithms 206

C. Desingularized Two-Layer Contour Dynamics 210

D. Numerical Determination of the Critical Points 212

8. References 214 
Guardando nel suo Figlio con l'Amore che l'uno e l'altro etternalmente spira, lo primo ed ineffabile Valore, quanto per mente e per loco si gira con tant'ordine fe', ch'esser non puote sanza gustar di lui chi ciò rimira.

Leva dunque, lettore, allalte ruote meco la vista, dritto a quella parte dove l'un moto e l'altro si percuote;

e lí comincia a vagheggiar nell'arte di quel maestro che dentro a sé l'ama, tanto che mai da lei l'occhio non parte.

Dante Alighieri

Paradiso, Canto $X$ 
NON SOLUM IN MEMORIAM SED IN INTENTIONEM

GiovanNi Polvani
$(1892-1970)$ 


\section{Prolegomena}

Strong, isolated and coherent vortical structures, among which Gulf Stream rings, "blocking patterns" in the atmosphere and the archetypical Jupiter Great Red Spot are familiar examples, are prominent features of many geophysical flows and consequently the understanding of their dynamics is a matter of great importance. At the same time, in recent years, great advances have been made in the study of the two-dimensional incompressible vorticity fields. Not only have new and powerful techniques been developed, but a whole new series of problems have been proposed and successfully solved, leading to many illuminating new insights.

On the basis of these two premises, the main objective of this thesis is the following: to extend the methods as well as the approaches that have proven so valuable to the study of two-dimensional rotational fluids to investigate the vorticity dynamics of a simple physical system (Phillips' two-layer model) that exhibits many of the fundamental properties of large scale geophysical flows. Before discussing our results it is however necessary to review a certain number of points, and that is the purpose of this introductory chapter.

More specifically, we proceed first to present the two-layer model in some detail and to make explicit the properties that make it a good candidate for the investigation of the vorticity dynamics of large scale geophysical flows; we will also expose its limitations (Section 1.1). The following section is dedicated to a brief review of coherent vortex structures in two-dimensional incompressible flows; the two-dimensional results have largely served us as paradigms for the investigation of the two-layer model (Section 1.2). We next explain the motivations behind the contour dynamical approach that we have almost exclusively adopted here (Section 1.3), and then present a synopsis of the work contained in this thesis (Section 1.4). The last part of this prolusory chapter is dedicated to a somewhat detailed exposition of the numerical algorithms that we have used throughout the thesis (Section 1.5). 


\subsection{The Two-Layer Model}

Our life is frittered away by details...Simplify,simplify!

Thoreau

The complexity of most geophysical flows is such that, if one is to make any progress towards the understanding of their dynamics, a specific strategy is needed to reduce the problems to tractable dimensions. We have, in this thesis, espoused the following point of view: rather than directing our attention immediately to any concrete geophysical situation (say, a Gulf Stream ring or the Great Red Spot) we have elected to investigate the dynamics of a separate and hopefully much simpler physical system which possesses several characteristics proper of the geophysical situations of interest to us.

With this strategy it is hoped that, having understood the physics of the simpler system, meaningful statements can be made with regard to the more complex geophysical context. The task of "exporting" the ideas learnt from the simpler system and determining if and to what extent they are relevent to any actual geophysical situation is a difficult and laborious one, and could easily make the object of another thesis. We have here limited ourselves to selecting a simple system whose properties make it very germane to large scale geophysical flows and to investigating its vortex dynamics in some depth.

The system we have chosen is the familiar quasi-geostrophic two-layer model on the f-plane, also referred to as Phillips' model. Although derived originally as a two-level model (Phillips 1954), we have adopted here the twolayer version first formulated by Pedlosky (1970). Since this model has been extensively used in the oceanographic and meteorological literature, it will suffice here to recall its principal characteristics (for a full derivation see Pedlosky 1979).

As shown in Figure 1.1, the physical system under consideration is composed of two stably stratified horizontal fluid layers of density $\rho$ and $\rho+\Delta \rho$, with $\Delta \rho<<\rho$. The thickness $\mathrm{H}_{1}$ (and $\mathrm{H}_{2}$ ) of the upper (and lower) layer is considered to be much smaller than the horizontal scale $L$ of the system; the fluid is thus always in hydrostatic equilibrium. The system rotates with an angular velocity $\omega$ large compared to the relative vorticity $U / L$ ( $U$ being the velocity scale), i.e. the Rossby number $\mathrm{Ro}=\mathrm{U} /(2 \omega \mathrm{L})$, is small compared to 1 ; this ensures that, at lowest order in Ro, the fluid is in geostrophic balance. 
Under these conditions, the motion in each layer is strictly two-dimensional (ct. the Taylor-Proudman theorem) and can be described by a streamfunction $\psi_{i}$ ( $\mathrm{i}=1$ and 2 for the upper and lower layer, respectively). The dynamics of the system is dictated by the statement of conservation of geostrophic potential vorticity $\Pi_{i}$ in each layer. After nondimensionalization, the two-layer model equations take the simple form:

$$
\left[\partial_{t}+J\left(\psi_{i}, \cdot\right)\right] \Pi_{i}=0
$$

where

$$
\begin{aligned}
& \Pi_{1}=\nabla^{2} \psi_{1}+\gamma^{2}\left(\psi_{2}-\psi_{1}\right) \\
& \Pi_{2}=\nabla^{2} \psi_{2}+\delta \gamma^{2}\left(\psi_{1}-\psi_{2}\right)
\end{aligned}
$$

and

$$
J(A, B)=\partial_{x} A \partial_{y} B-\partial_{y} A \partial_{x} B
$$

and $\nabla^{2}$ is the two-dimensional Laplacian operator. Note that only two nondimensional parameters appear in this formulation: the first one $\delta=\mathrm{H}_{1} / \mathrm{H}_{2}$ is the ratio of the upper to lower layer thickness (when the fluid is at rest) and the second one $\gamma=L / L_{R}$ is ratio of the length scale to the radius of deformation $L_{R}$ defined by:

$$
\mathrm{L}_{\mathrm{R}}=(2 \omega)^{-1}\left(\frac{\mathrm{g} \Delta \rho \mathrm{H}_{1}}{\rho}\right)^{1 / 2}
$$

The parameter $\gamma$ can be intuitively understood as the rigidity of the interface between the two layers. In particular, when $\gamma=0$ (which corresponds to a perfectly rigid interface) the two layers become uncoupled and behave as two independent two-dimensional fluids obeying the two-dimensional Euler equations (Lamb 1945, Landau and Lifschitz 1959):

$$
\left[\partial_{\mathrm{t}}+\mathrm{J}(\psi, \cdot)\right] \zeta=0 \quad \text { with } \quad \zeta=\nabla^{2} \psi
$$


This model represents the stratification as two coupled layers in which the advection is two-dimensional, and it is able to retain a linear relationship between the streamfunction and the geostrophic potential vorticity, as can be seen from (1.1b). Thus, given the vorticity fields $\Pi_{i}$, the streamfunctions can be obtained by solving the linear system (1.1b), and are given by:

$$
\psi_{i}(x, y)=\frac{1}{2 \pi} \sum_{j=1}^{2} \iint \Pi_{i}(\xi, \eta) G_{i j}(r) d \xi d \eta
$$

where

$$
r=\left[(x-\xi)^{2}+(y-\eta)^{2}\right]^{1 / 2}
$$

and $G_{i j}$ is the Greens' function for the effect in layer $i$ of a point vortex in layer $j$. The four Greens' functions needed can be derived in a straightforward way from (1.1b) and are found to be:

$$
\begin{aligned}
& \mathrm{G}_{11}=\frac{\delta}{1+\delta} \log r-\frac{1}{1+\delta} \mathrm{K}_{0}(\Gamma r) \\
& \mathrm{G}_{21}=\frac{\delta}{1+\delta} \log r+\frac{\delta}{1+\delta} \mathrm{K}_{0}(\Gamma r) \\
& \mathrm{G}_{12}=\frac{1}{1+\delta} \log r+\frac{1}{1+\delta} \mathrm{K}_{0}(\Gamma r) \\
& \mathrm{G}_{22}=\frac{1}{1+\delta} \log r-\frac{\delta}{1+\delta} \mathrm{K}_{0}(\Gamma r)
\end{aligned}
$$

where

$$
\Gamma=\gamma(1+\delta)^{1 / 2}
$$

and $\mathrm{K}_{0}$ is the modified Bessel function of order zero.

The system described by (1.1) is the simplest that incorporates both geostrophic and hydrostatic balances as well as baroclinicity, some of the most important characteristics of large scale geophysical flows. The principal limitation of this model rests in the fact that the layer thicknesses are only allowed to change by $O(R o)$ amounts with respect to their values when the fluid is at rest. Thus a phenomenon such as the formation of fronts cannot take place in such a system. 
The other major simplifications are the absence of a planetary gradient of potential vorticity (the $\beta$ effect) and of dissipation. These were motivated by the following two reasons: in the first place, as will be shown in chapters 2 and 3 , the inviscid f-plane dynamics is rich enough that it deserves a thorough investigation by itself. More fundamentally, however, the techniques that we intend to use are not well suited to handle such effects. How and why this is the case will become evident after the discussion of section 1.3.

As we have already pointed out, the quasi-geostrophic two-layer model consists of two coupled two-dimensional layers, and for which the inverse of the deformation radius (squared) plays the role of the coupling constant. Since the primary objective of this thesis has been to consider how two-dimensional vortex dynamics is modified by the presence of stratification, it will be wise, before proceeding to the study of the coupled system, to recall the principal results that are known about the vortex dynamics of two-dimensional fluids.

\subsection{A concise review of Euler results}

Qien mira lo pasado, lo porvenir advierte.

Lope de Vega

Although the equations describing two-dimensional incompressible flows the Euler equations for the inviscid case and the Navier-Stokes equations for the viscous one (the difference being a $v \nabla^{2} \psi$ term to account for dissipation) - have been known for several hundred years, progress towards the understanding of the nonlinear dynamics they generate has been quite slow until the advent of computers and the availability of high resolution numerical simulations.

These have not only provided access to complicated new solutions but, more importantly, have paved the way by helping to formulate the essential questions of two-dimensional hydrodynamics. This synergetic interaction has already been the subject of a number of reviews to which the reader is referred for a detailed description (Zabusky 1986, 1985, 1984, 1981 and references therein).

Perhaps the clearest way of illustrating this is to briefly recall the by now classic - although quite recent - numerical experiments of McWilliams (1984). He

To observe the past is to take warning for the future. 
simulated the decay of a two-dimensional velocity field using a 1282 dealiased pseudospectral code (with $\nabla^{6} \psi$ hyperviscosity to confine the damping to the smallest scales in the flow). The initial condition was taken to be a broad-banded spectrum peaked near wavenumber 6 with a random Gaussian initialization for each Fourier component.

The evolution of the vorticity field is shown in Figure 1.2 (reprinted from McWilliams 1984). Although the analysis of the energy spectrum reproduces the familiar cascades of 2D turbulence, i.e. enstrophy to small scales and energy to large scales - for a thorough review see Kraichnan and Montgomery (1980) - it is unable to capture the remarkable development of intermittency in the vorticity field that corresponds to the emergence of isolated coherent vortex structures (an analysis of the fourth moment - the kurtosis - is necessary to detect the highly non-Gaussian distribution of the vorticity field) $t$.

As McWilliams describes, the essential mechanism for the condensation of vorticity into isolated entities is the merger of two structures of equal signed vorticity into a single larger one; this process is repeated until the number of vortices is so low that encounters become extremely rare. If they have opposite sign vorticity the encounter can lead to binding, whereby a dipolar structure is created. McWilliams also noted that after a long enough time the vortices tend to relax into an axisymmetric configuration through a process termed "filamentation".

More generally, the fundamental questions of $2 \mathrm{D}$ vortex dynamics that have emerged from this and similar studies can be concisely formulated as follows:

a. What are the stationary configurations of vorticity and which of these are stable?

b. What are the mechanisms by which two like-signed (oppositesigned) regions of vorticity merge (bind)?

c. How does the axisymmetrization process occur, and what exactly is the role of filamentation?

† That the energy spectrum, by discarding the phase information, is a particularly inadequate tool for detecting such phenomena is by now a generally recognized fact. Hank Stommel, with his customary wit, has remarked that "a power spectrum of a Beethoven symphony would be rather an oversimplification too; maybe just enough to decipher the key"! (quoted in Armi and Flament 1985) 
(It is useful to note that, in the jargon of two-dimensional turbulence, merger corresponds to the well known reverse cascade of energy to the large scales, while the filamentation process corresponds to the forward cascade of enstrophy to high wavenumbers). It is probably fair to say that all the above matters have been investigated with success over the past decade, although many questions still need to be resolved. A short summary of the main results follows.

a. Because of the symmetry of the Laplacian operator any function $\psi=\psi(r)-r$ being the polar distance from the origin - is an exact solution of the Euler equations in two-dimensions. However, fully axisymmetric fields are not very interesting since the Jacobian term in (1.2) vanishes identically; thus, in some sense, such fields are in fact little more than very special linear solutions that, due to the symmetry of the system, happen to satisfy the fully nonlinear equations. Moreover, the study of the stability for any specific radial function is a complicated problem and only a few cases have been explored (see e.g. Legras and Carton, 1988).

The simplest weak (i.e. discontinuous in the second derivative of $\psi$ ) analog of the smooth axisymmetric solutions is known as the Rankine vortex (Lamb 1945): it is a circular patch of uniform vorticity embedded in an irrotational flow. Since the work of Lord Kelvin (1880) it is known to be stable to infinitesimal perturbations of all wavelengths.

Its nonlinear stability has only recently been investigated by Wan and Pulvirenti(1985) and by Dritschel (1988). Recall that nonlinear stability is only meaningful with respect to a specified norm, so that a configuration could be stable in one norm but not in another. For the Rankine vortex, Wan and Pulvirenti (1985) have shown that the Rankine vortex is nonlinearly stable in a norm that measures the area of the rotational fluid beyond the equilibrium circular boundary, whereas Dritschel (1988) has provided computational evidence of its instability with respect to a norm measuring arc-length. Taken together these results suggest that a perturbed Rankine vortex remains circular in a coarse-grained sense, but with a boundary that becomes increasingly convoluted and ill-defined. 
Among weak solutions, the Kirchhoff ellipse (Lamb 1945) is the simplest one to exhibit a noncircular symmetry: it consists of an elliptic patch of constant vorticity rotating without change in shape in an irrotational background with a constant angular velocity $\Omega_{\mathrm{k}}$ given by the expression:

$$
\Omega_{\mathrm{k}}=\mathrm{q} \frac{\lambda}{[1+\lambda]^{2}}
$$

where $q$ is the value of the vorticity inside the ellipse and $\lambda$ is the aspect ratio (the ratio of the minor to the major axis, note that $0 \leq \lambda \leq 1$ ). The Kirchhoff ellipse is known to be linearly unstable to infinitesimal perturbations when the aspect ratio is less than $1 / 3$ (Love 1893).

Looking at the Kirchhoff ellipse as the finite amplitude extension of an $m=2$ ( $m$ being the azimuthal wavenumber) perturbation on a Rankine vortex, one is led to consider the existence of finite amplitude solutions for values of $m>2$. These were found numerically by Deem and Zabusky (1978a and b), and were given the name "V-states". Their linear stability was studied by Burbea (1980) and Burbea and Landau (1982). More generally, the term V-state describes any exact nonlinear weak solution of the inviscid Euler equations consisting of any number of finite area regions of piecewise constant vorticity (or potential vorticity, in the case of the two-layer model equations).

Dipolar vortices consisting of two patches of constant equal and opposite vorticity (also termed "translating V-states" or "modons") were numerically found by Deem and Zabusky (1978a and b) and independently by Pierrehumbert (1980); the delicate question of the existence and shape of the limiting V-states was settled by Wu et al (1984). In addition, the so called "Batchelor couples" (Lamb 1945, Batchelor 1967) are analytical dipolar exact solutions of the Euler equations in which the vorticity is linearly related to the streamfunction within a certain radius (the radius of the couple) $\dagger$. They are, however, discontinuous in the first derivarive of the vorticity.

Finally, among a number of other $V$-state solutions that have been discovered in recent years we mention the $\mathrm{N}$-fold multiply connected rotating $\mathrm{V}$ states calculated by Dritschel $(1985,1986)$ who also studied their linear stability and their nonlinear evolution (the $\mathrm{N}=2$ states had been previously reported by

† Although the first reference to these solutions appears in Lamb, the name of "Batchelor couples" is found most frequently in the literature. 
Saffman and Szeto 1980, and Zabusky 1981b), the vortex street of finite-core vortices (Saffman and Schatzman 1982, Kida 1982, Meiron et al. 1984) and the asymmetric doubly-connected rotating V-states (Zabusky 1981b).

It should be kept in mind that point vortices (and combinations thereof) are also solutions of the Euler equations. The idea of singularizing the continuum is originally due to Kirchhoff, and the singularized Euler equation can be shown to be a Hamiltonian system with as many degrees of freedom as there are point vortices and can exhibit both regular and stochastic motion (for an extensive review of this subject the reader should consult Aref 1983).

b. Of the two elementary interactions, merger and binding, it is the merger process that has received the largest attention, and this can be attributed mostly to the fact that it plays the truly essential role in the emergence of single-signed coherent vortices (binding is, on the contrary, a much more infrequent event and this is reflected in the relatively small number of dipoles formed compared to monopoles).

Recall that two point vortices simply rotate about their common centroid (i.e. the center of vorticity) while their distance remains constant. Finite area vortex regions (not necessarily of constant vorticity) on the other hand exhibit a much richer behavior (this is a typical example in which desingularization is necessary to reveal qualitatively new dynamics). The pioneering work, using a vortex-in-cell method, is due to Roberts and Christiansen (1972) and Christiansen and Zabusky (1973) while later studies (Deem and Zabusky 1978a and b, Zabusky et al. 1979, Overman and Zabusky 1982a, Jacobs and Pullin 1985) used a contour dynamical algorithm.

When two circular regions of uniform vorticity and radius $\mathrm{R}$ are placed such that their centers are separated by a distance $D$, one of two things occurs depending of the value of the (nondimensional) ratio $d_{c}=D / R$ : if $d_{c}<3.3$ (approximately) the two vortices approach and wrap around each other forming a single vortex, i.e. they merge. If, on the other hand, $d_{c}>3.3$ the two vortices simply rotate around one another undergoing regular pulsations in their shape but without ever coalescing into a single vortex. A classic illustration of these two processes is shown in Figures 1.3a and $b$ (from Roberts and Christiansen, 1972).

The crucial question here is, of course, to understand how the critical value of 3.3 comes about and to try to predict it without the full numerical simulations. Saffman and Szeto (1980), using extremely simple arguments, were able to predict 
a value of 3.0 and, with more sophisticated methods, a value of 3.16 . Their approach is based on the consideration of the properties of the $\mathrm{N}=2$ corotating $\mathrm{V}$ states. We have generalized their approach considerably and have shown that, in fact, there is a very close connection between the existence of V-states and the merger criterion (we will review their work in detail in section (2.2.3).

More recently Melander, Zabusky and Styczek (1984 and 1986) have introduced an elliptically desingularized model for the two-dimensional Euler equations (the "moment model") that they have applied to the symmetric merger problem with success. With the moment model, the merger process can be reduced to a four dimensional integrable Hamiltonian system that can be solved analytically, yielding a value of 3.2 for the critical merger distance. The success of the moment model is, perhaps, one of the most beautiful examples of the creativity generated by the synergetic interaction between theoretical and computational research, and it represents, in the spirit of von Neumann, a significant analytical penetration into the two-dimensional turbulence problem.

Finally, of the several other types of interactions that have been investigated in recent years, we recall the asymmetric merger problem (Melander at al. 1987), the merger arising from unstable perturbed V-states (Overman and Zabusky $1982 \mathrm{~b}$ ) and the scattering of Batchlelor couples (McWilliams and Zabusky 1982, Couder and Basdevant 1986).

c. The so called "axisymmetrization principle" was first formulated by Melander and coworkers (1987a) who studied the evolution of one isolated elliptical vortex in a weakly dissipative two-dimensional fluid using a high resolution dealiased pseudospectral code. They showed computationally that a spatially smooth vortex relaxes inviscidly towards axisymmetry on a circulation time scale - an example is shown in Figure 1.4 (reprinted from Melander et al. 1987a). This investigation has provided an understanding of the already mentioned discovery of McWilliams (1984) that most monopolar vortices emerging spontaneously from a turbulent vorticity field are very nearly axisymmetric.

It is important to recall that a top hat elliptical vortex (i.e. a Kirchhoff) ellipse is an exact solution of the Euler equations and, unless perturbed, maintains its initial eccentricity. The fact that axisymmetrization is a truly inviscid process has been confirmed by the contour dynamical computations of Dritschel (1988) who has observed the axisymmetrization of a set of nested elliptical regions of constant vorticity. 
The studies of Melander, Zabusky and McWilliams (1987 a and b, 1988) have.also brought to light the powerful insights that can be obtained from the consideration of the topology of the co-moving streamfunction, i.e. the streamfunction as seen from a frame rotating or translating with the coherent vortex structure. In particular, the location of centers, saddle points and separatrices with respect to the vorticity distribution was shown to be crucial in determining the evolution of the flow, and in predicting the occurence of strongly nonlinear events such as merger and filamentation.

\subsection{Contour Dynamics}

Et vera incessu patuit dea. ${ }^{\dagger}$

Virgil

Having chosen the quasigeostrophic two-layer model as the physical system that will be the object of our study in this thesis, and having been exposed to a great wealth of techniques and results for the vortex dynamics of the Euler equations in two dimensions, we are now confronted with the question of solving the equations (1.1). At the risk of offending the reader, we point out that, in spite of the linearity of (1.1b), system (1.1) is extremely complicated: in addition to a nonlinear advection similar to the one of the Euler equations, it involves the nonlinear coupling of the two layers. In order to motivate the method that we have opted to use, we start by recalling two approaches that have proven useful in the past.

One possible avenue of analytical progress is to find (by unspecified means) simple exact solutions of (1.1) and to study their stability. Such an approach was taken, for instance, by Pedlosky (1985) and more recently by Flierl (1988). It suffers, however, from two severe limitations: first, only a small set of exact analytical solutions can be found (simple jets, vorticity fronts or circular vortices). Secondly, and more importantly, only the linear - or at most (with some courage and a lot of patience!) the weakly nonlinear - stability of these exact solutions can be studied analytically. This approach will be of limited applicability

$\dagger$ And in her walk it showed, she was in truth a goddess. 
here since the phenomena of interest to us are fundamentally nonlinear, i.e. nonlinear at $O(1)$.

In order to address such problems an alternative approach to the solution of (1.1) has been the direct numerical simulation using finite difference or, more commonly nowadays, pseudospectral schemes. Although this second strategy does allow one to tackle the full nonlinearity, it presents the inconvenience of requiring large amounts of computer memory and CPU time (typically, a CRAY would be needed to perform runs at any reasonable resolution). Moreover the simulation of the full system often obscures some of the simpler aspects of the dynamics.

What is needed is a technique to reduce the complexity of the system while retaining the fundamental nonlinearity. We believe that contour dynamics (Zabusky, Hughes and Roberts 1979) provides such a via media, in as much as it allows one to simplify the dynamics and at the same time tackle problems that are nonlinear at $O(1)$. The central idea of contour dynamics is the following: simplify the system not by linearizing, but by reducing the complexity of the vorticity field through a discretization of the vorticity levels.

The cleverness of this approach becomes apparent once the following crucial observation is made: in a two-dimensional fluid (or a multilayer one such as the two-layer model) the gradients of vorticity (or potential vorticity) are the locations where action takes place. If one then approximates a smooth (i.e. differentiable) vorticity field with a set of discrete nested regions of constant vorticity, one confines the gradients of vorticity to the contours delimiting these regions. By this simple device one has in fact reduced a two-dimensional problem to a one- dimensional one, since the field is then totally described by the location of the contours.

It is important to stress that, from a mathematical point of view, such an approach yields exact solutions to (1.1), and not approximations to the exact solutions which would be obtained, say, with a Galerkin scheme. By adopting the contour dynamical approach one effectively restricts one's attention to solutions that, although exact, are discontinuous in the second derivatives of the streamfunction.

In practice eventually, one has to implement a numerical algorithm to determine these weak solutions, and at that stage approximations are necessary to carry out the integration numerically. However the numerical solutions obtained via contour dynamics approximate the much simpler weak exact solutions and not 
the more complicated infinitely differentiable solutions that one tries to approximate, for instance, with a spectral code. Contour dynamics is therefore much more than simply "another numerical scheme"; it reduces the complexity of the dynamics by limiting one's scope to vorticity fields that are intrinsically simple (although discontinuous) ${ }^{\dagger}$.

From a physical standpoint, however, it is useful to consider the contour dynamical approach as an approximation to the continuous problem whereby a smooth vorticity gradient is represented by a series of vorticity jumps. The lowest order of approximation replaces the smooth vorticity gradient with a single vorticity step, the next order with two steps, and so on. Thus the continuous solutions can be approximated to any degree of accuracy by including a sufficient number of vorticity levels. In practice, however, the computations become quite cumbersome when more than a few levels are considered, and, in fact, the strength of the contour dynamical approach rests effectively in its ability to reproduce the dynamics of the continuous system with a very small number of levels.

As a matter of fact, all of the computations in this thesis were carried out with a single vorticity step, but there is little doubt that, for the initial value problems, the main qualitative conclusions would have emerged had more levels (or even the continuous system) been considered. We base this assertion on several comparative studies that were carried out with the Euler equations which we have reviewed in the previous section.

As far as the $\mathrm{V}$-states are concerned, their connection to smooth analogs is not always as clear, mostly because smooth analogs are usually not known. In most cases it is reasonable to suspect that smooth analogs exist, especially when they are found to emerge spontaneously in spectral simulations. One such example is provided by the tripolar vortex structures which will be examined in detail in Chapter 6 . It is however important to keep in mind that there exist "singular" situations in which the results of a single step contour dynamical calculation are not representative of the continuous system (the Kirchhoff ellipse vs the axisymmetrization principle is a good example of this).

A further reason that contributes to making contour dynamics especially appealing as a tool for studying the kinds of problems we have mentioned is the fact that it is, to our knowledge, the only technique that allows one to investigate

T We are referring here to low to moderate resolution calculations such as the ones we have performed in this thesis. At very high resolution the vorticity fields become extremely complicated as was shown by Dritschel (1988a). 
the dynamics of truly isolated structures, since it particularly well suited to hadle situations in which the fluid is unbounded in all directions. Most spectral methods are designed for periodic domains, and finite-difference or finite-element methods also need periodic or finite domains. It should be noted that contour dynamics too can deal with finite or periodic domains, provided the Greens' functions are chosen to satisfy the appropriate boundary conditions (see e.g. Stern and Pratt 1985).

Before describing in detail the numerical algorithms that we have used, we wish to conclude this general discussion of contour dynamics by pointing out the limitations from which it suffers. As is often the case, it turns out that its greatest strength is at the same time its greatest weakness. Because the idea of confining the vorticity gradients to contours is at the heart of its power of simplification, contour dynamics is inherently incapable of dealing with situations where the vorticity gradient is nonzero over a two-dimensional surface. Such is the case, for instance, for many geophysical situations which necessitate the presence of a planetary vorticity gradient. Note that "mean flows" are not totally excluded; provided their shear is linear (so that their vorticity gradient vanishes) they can be handled easily. However it is crucial, for the method to be applicable, that all vorticity gradients be confined along contours.

The fact that contour dynamics is not well suited to deal with dissipative systems is also a handicap. However, the kind of phenomena that we will discuss in this thesis are essentially inviscid ones - e.g. the merger problem - and the comparison with viscous spectral calculations show that, when the viscosity is small enough, contour dynamics is a very appropriate tool to use. Moreover, although to the best of our knowledge no one has attempted to do this yet, there may be ways of incorporating some form of dissipation by allowing the areas enclosed by the contours to increase and the vorticity to change with time.

This handicap, however, can become a most valuable advantage if one is interested in reproducing the truly inviscid dynamics of a system. In that case contour dynamics offer a power of resolution that is unparalleled by any other technique. Thus, for instance, the recent work of Dritschel (1988a, b) has revealed details of structures in inviscid two-dimensional flows, whose complexity was hitherto unsuspected and beyond the grasp of even the highest resolution spectral simulations.

In the final analysis we believe that the special appeal of the contour dynamical method resides in its being particularly appropriate in addressing the 
fundamental questions of vortex dynamics that have been successfully investigated for two-dimensional incompressible flows and that we have considered in the context of the quasi-geostrophic two-layer model.

\subsection{Synopsis}

\section{Muß es sein? Es muß sein. ${ }^{\dagger}$}

Beethoven

After these perhaps protracted but necessary remarks, we hope the readers will find themselves in a position of being able to more fully appreciate the relevance of the problems we have decided to address in this thesis. In our attempt to understand the vortex dynamics of the two-layer model, we have decided to increase the complexity of the system in a gradual manner from the purely two-dimensional case. We have thus first limited our attention to the case in which geostrophic potential vorticity is non-zero in the upper layer alone. This was done in order to assess to what extent the presence of an active lower layer of finite depth and constant potential vorticity influences the vortex dynamics in the upper layer. The results of this investigation are presented in Chapter 2.

We have then considered situations where the geostrophic potential vorticity is non-zero in both layers, and Chapter 3 is devoted to the study of some of the simplest possible configurations. In general, we have given the greatest attention to the merger problem and its two-layer counterpart, the "alignment" problem. We have also determined a number of one and two-layer V-states which have provided a valuable means of quantifying some of the qualitative results obtained from the initial value calculations.

An important finding, that emerged during the study of the two-layer geostrophic problems just mentioned, is the importance of the location of critical (hyperbolic) points of the streamfunction in a frame of reference rotating (or translating) with the velocity of the coherent vortex structures. These points are found to play a major role in determining the regions in parameter space where stationary solutions (V-states) exist.

† Must it be? It must be. 
Reverting to the simpler two-dimensional context, we show, in Chapter 4, how the critical points of the corotating streamfunction are also deeply related to the process of filamentation. For the simple case of a perturbed Kirchhoff ellipse we derive, from a geometrical analysis of the corotating fields, an expression for the onset time of filamentation that compares well with fully nonlinear numerical simulations.

The Addenda contain a number of smaller problems that should also be considered further advances in the understanding of more complicated vorticity configurations, but where the complexity is added by increasing the number of contours while retaining the two-dimensional assumption. In Addendum A, we present the first (and to our knowledge) only known analytic time-dependent nonaxisymmetric family of multi-contour solutions to the two-dimensional Euler equations. Addendum B illustrates an example where the linear stability of a 2contour vortex can be shown to be connected with the location of the critical points of the associated corotating streamfunction. Finally, in Addendum C, we report on a new set of $\mathrm{V}$-states for the two-dimensional Euler equations: they are the finitearea constant-vorticity analogs of the tripolar coherent vortex structures that have recently been observed in very high resolution numerical simulations of twodimensional turbulence. 


\subsection{Algorithmst}

$\circ \beta \operatorname{\beta i} \beta \rho \alpha \chi v \varsigma, \eta \delta \varepsilon \tau \varepsilon \chi v \eta \mu \alpha \chi \rho \eta$.

Hippocrates

We describe in this section the two contour dynamics algorithms that we have used repeatedly, with a number of variations, throughout this thesis: the first one, which may rather deserve the name of "contour statics", has served to determine a variety of $\mathrm{V}$-state solutions; the second one was used to compute inital value problems. They share the way in which the velocity field is calculated from the location of the contours delimiting the closed regions of constant vorticity. It is this technique that we proceed to decribe next.

For the sake of generality, consider a vorticity field made up of $N_{j}$ regions $D_{j}(n)$, where $j=1,2$ refers to the upper and lower layer respectively and $n=1, \ldots, N_{j}$. Let $\partial D_{j}{ }^{(n)}$ designate the contour enclosing $D_{j}(n)$ and let $\left\{\Pi_{j}(n)\right\}$ be the jump in potential vorticity across $\partial D_{j}(n)$, namely $\left\{\Pi_{j}(n)\right\}=\Pi_{j}$ (outside $\left.D_{j}(n)\right)-\Pi_{j}$ (inside $\left.D_{j}(n)\right)$. Since $\Pi_{j}$ is constant inside each $D_{j}(n)$ it can be taken outside the integral of (1.3) which then simplifies to :

$$
\psi_{i}(x, y)=\frac{1}{2 \pi} \sum_{j=1}^{2} \sum_{n=1}^{N_{j}}\left\{\Pi_{j}(n)\right\} \iint_{D_{j}(n)} G_{i j}(r) d \xi d \eta
$$

Expressions for the velocity in layer $i$ as contour integrals over the boundaries $\partial \mathrm{D}_{j}(n)$ are obtained by differentiating (1.6) and applying Greens' theorem to yield:

$$
\left[u_{i}, v_{i}\right]=\left[-\partial_{y} \psi_{i}, \partial_{x} \psi_{i}\right]=-\frac{1}{2 \pi} \sum_{j=1}^{2} \sum_{n=1}^{N_{j}}\left\{\Pi_{j}(n)\right\} \int_{\partial D_{j}(n)} G_{i j}(r)[d \xi, d \eta]
$$

In practice the contour integration in (1.7) has to be carried out numerically, and this is done by substituting the integral with a summation. Each contour is represented by a set of $M_{j}^{(n)}$ nodes $\left\{x_{j}^{(n)}(m), y_{j}^{(n)}(m)\right\}$ - with $m=1, \ldots, M_{j}(n)$. Moreover the nodes are connected by straight line segments (more sophisticated versions of contour dynamics use various sorts of polynomial interpolations to represent

† Since this section is of a very technical nature, we encourage the reader who may be rather uninterested in the computational aspects of this work, to proceed directly to Chapter 2.

$¥$ The life so short, the craft so long to learn. 
contours, e.g. Dritschel 1988). The discrete form of (1.7) that gives the velocity at any point $(x, y)$ in layer $i$ becomes:

$$
\left[u_{i}, v_{i}\right](x, y)=\frac{1}{2 \pi} \sum_{j=1}^{2} \sum_{n=1}^{N j}\left\{\prod_{j}(n)\right\} \sum_{m=1}^{M_{j}(n)} G_{i j}\left(r{ }_{j}^{(n)}(m)\right)\left[\delta \xi_{j}^{(n)}(m), \delta \eta_{j}^{(n)}(m)\right]
$$

where

$$
\begin{gathered}
\delta \xi_{j}^{(n)}(m)=x_{j}^{(n)}(m+1)-x_{j}^{(n)}(m) \quad \delta \eta_{j}^{(n)}(m)=y_{j}^{(n)}(m+1)-y_{j}^{(n)}(m) \\
r_{j}^{(n)}(m)=\left[\left(x_{j}^{(n)}\left(m+\frac{1}{2}\right)-x\right)^{2}+\left(y_{j}^{(n)}\left(m+\frac{1}{2}\right)-y\right)^{2}\right]^{1 / 2}
\end{gathered}
$$

and

$$
x{ }_{j}^{(n)}\left(m+\frac{1}{2}\right)=\frac{1}{2}\left[x_{j}^{(n)}(m+1)+x_{j}^{(n)}(m)\right] \quad y{ }_{j}^{(n)}\left(m+\frac{1}{2}\right)=\frac{1}{2}\left[y_{j}^{(n)}(m+1)+y_{j}^{(n)}(m)\right]
$$

We have used formula (1.8) without exception throughout this thesis. Earlier versions of contour dynamics algorithms (Zabusky, Hughes and Roberts 1979, Stern and Pratt 1985, 1986, Jacobs and Pullin 1985) used a more complex expression that was intended to analytically desingularize the logarithmic divergences of the Greens' functions by evaluating separately the contributions from the two nearest segments on the contour.

[It turns out that there is a much simpler way than Pratt and Stern's to desingularize the evaluation of the velocities from (1.7) and it is presented in detail in Appendix C. We have tested it against (1.8) for both for the static and dynamic calculations we have performed, but have found extremely small differences at the somewhat low resolutions we have used in this thesis.]

\subsubsection{Iterative Scheme for V-States}

We now describe the second order iterative algorithm that we have used in the determination of several types of one and two-layer $\mathrm{V}$-states. We have essentially generalized the method of Wu et al. (1984) to make it applicable to different geometries and to more than one layer. We will give here the general outline and point out the specifics for each type of V-state in the correpsonding sections and appendices. 
Usually, because of symmetry considerations, only a portion of the V-state needs to be calculated and the rest can be obtained by rotation and/or translation. Thus, for example, to find symmetric translating dipolar V-states, only one half of one vortex of the modon has to be computed; this is the dotted portion of the contour shown in Figure 1.5. This segment is discretized into $\mathrm{N}$ intervals. The endpoints - designated by crosses - are given, and are part of the free parameters of the problem. The unknowns of the problem are the the locations of the $\mathrm{N}-1$ nodes between the endpoints and $\mathrm{V}$, the linear velocity with which the $\mathrm{V}$ state translates (for rotating $V$-states, an angular velocity $\Omega$ has to be determined).

To simplify matters, the $\mathrm{N}-1$ nodes are placed at given and fixed (not necessarily equal) angular intervals between the endpoints so that only their radial distances from the center of the contour - designated $R_{i}(i=2, N-1)$ - need to be found. The number of unknowns is then reduced to $N$ : the linear or angular velocity plus the $\mathrm{N}-1$ values of the $R_{i}$ 's. The $N$ equations needed are obtained by enforcing that, on the boundaries, the normal velocity of the fluid be equal to the normal velocity of the vortex boundaries themselves. For the translating states this is expressed by:

$$
(v-V) \frac{d x}{d \theta}-u \frac{d y}{d \theta}=0
$$

and for the rotating states one has:

$$
v \frac{d x}{d \theta}-u \frac{d y}{d \theta}+\frac{1}{2} \Omega \frac{d R^{2}}{d \theta}=0
$$

where $\theta$ is the polar angle measured with respect to the center of the contour. In discretized form these become:

$$
\left(v_{i+1 / 2}-V\right)\left(x_{i+1}-x_{i}\right)-u_{i+1 / 2}\left(y_{i+1}-y_{i}\right)=0 \quad \text { for } \quad 1 \leq i \leq N
$$

while for the rotating states it becomes:

$$
v_{i+1 / 2}\left(x_{i+1}-x_{i}\right)-u_{i+1 / 2}\left(y_{i+1}-y_{i}\right)+\frac{1}{2} \Omega\left(R_{i+1}^{2}-R_{i}^{2}\right)=0 \quad \text { for } \quad 1 \leq i \leq N
$$

where $i+1 / 2$ designates the midpoint between node $i$ and node $i+1$ and the index $i$ goes from 1 to $\mathrm{N}$. The velocities at the midpoints are calculated using the sums given in (1.8). If the $N$ equations (1.9) are added, it is possible to solve for $V$ or $\Omega$ 
as a function of the locations of the nodes alone (since the velocities are also functions of the node positions); however this expression depends on the particular geometry of the V-state and the precise form of (1.9), and cannot be given in general. For example, for symmetric translating $V$-states, one has:

$$
V=\frac{1}{\left(x_{A}-x_{B}\right)} \sum_{i=1}^{N}\left[v_{i+1 / 2}\left(x_{i+1}-x_{i}\right)-u_{i+1 / 2}\left(y_{i+1}-y_{i}\right)\right]
$$

where $x_{A}$ and $x_{B}$ are the $x$-coordinates of the endpoints. For each type of $V$-state a formula analogous to $(1.10)$ allows one to calculate the linear or angular velocity from the positions of the nodes alone.

The $\mathrm{N}$ equations (1.9) can, moreover, be expressed as a tridiagonal system for the unknown $R_{i}$ 's by sustituting the expressions for $x_{i}$ and $y_{i}$ in terms of the polar coordinates $R_{i}$ and $\theta_{i}$. This substitution, after rearrangement, leads to $N$ equations of the form:

$$
R_{i}=F_{i+1 / 2} R_{i+1} \quad \text { for } \quad 1 \leq i \leq N
$$

and, alternatively:

$$
R_{i}=\frac{1}{F_{i-1 / 2}} R_{i-1} \quad \text { for } \quad 2 \leq i \leq N+1
$$

where again the F's are different for each type of V-state. They depend on the specific geometry and are functions of the the velocities and $V($ or $\Omega$ ); e.g. for the symmetric translating $\mathrm{V}$-states are given by:

$$
F_{i+1 / 2}=\frac{u_{i+1 / 2} \sin \theta_{i+1}-\left(v_{i+1 / 2}-v\right) \cos \theta_{i+1}}{u_{i+1 / 2} \sin \theta_{i}-\left(v_{i+1 / 2}-v\right) \cos \theta_{i}}
$$

Recall that the values $\theta_{i}$ of the angular positions of the nodes are fixed throughout the calculation. Combining (1.11a) and (1.11b) we build a tridiagonal system for the $\mathrm{N}-1$ unknown radial distances $R_{i}$ (recall that $R_{1}$ and $R_{N+1}$ correspond to the endpoints which have been chosen at the outset):

$$
\frac{1}{2} \frac{1}{F_{i-1 / 2}} R_{i-1}-R_{i}+\frac{1}{2} F_{i+1 / 2} R_{i+1}=0 \quad 2 \leq i \leq N
$$


After calculating the velocities with (1.8) and $V$ with the appropriate form of (1.10) using one set of values for the $R_{i}$ 's, the solutiont of (1.13) yields new values for the $R_{i}$ 's from which a new $V$ (or $\Omega$ ) and new velocities can calculated. In practice, as was found by Wu et al. (1984), to insure convergence one must use a relaxation procedure after each iteration, i.e. the new $R_{k}$ 's are "mixed in" with a certain proportion of the old ones before the next iteration is performed.

In summary, the iterative procedure goes as follows:

i. Start with an initial guess of the boundary shape (the $R_{k}$ 's).

ii. Calculate the velocities at the midpoints using (1.8).

iii. Evaluate $V($ or $\Omega$ ) from (1.10).

iv. Obtain the F's from (1.12).

v. Solve the tridiagonal system (1.13) for the new $R_{k}$ 's.

vi. Repeat steps ii to $v$ until some convergence criterion is satisfied.

The convergence criterion we have chosen is basically a test of whether the $L_{1}$-norm of the difference between two successive iterations is smaller that some given threshold. More specifically we terminate a run when the condition:

$$
\sum_{i=1}^{N+1}\left|R_{i}^{(n+1)}-R_{i}^{(n)}\right|<\varepsilon
$$

is satisfied. In most cases we have found that, with $O(100)$ points per contour convergence is achieved in less than $O(100)$ iterations for $\varepsilon=O\left(10^{-5}\right)$.

It is important to remark that the curvature of the solution that is being found has a great deal of impact on the convergence rate of the algorithm. Regions of high curvature are difficult to obtain unless a sufficient number of nodes is used to resolve them accurately. As is the case for the $\mathrm{V}$-states that have already been found, regions of high curvature seem to arise as the parameters approach some limiting values beyond which no solution exists. The analysis of these limiting cases may require a higher order algorithm (see Wu et al. 1984).

As we will have the opportunity to present in detail in Chapters 2 and 3 , an important new result of our work has been the discovery that the existence and geometry of the limiting V-states is dictated, in some cases, by the presence, in the co-moving streamfunction, of critical points that become very close to the contours and are responsible for the formation of cusps. It turns out that one is often

T The tridiagonal system (1.3) is solved using a Crout reduction (ct. for instance Carnahan, Luther and Wilkes 1969) which is both simple to encode an relatively fast. 
interested in just such limiting cases, because one is trying to determine the critical value of some parameter beyond which no $\mathrm{V}$-state exists.

The strategy we have adopted in this thesis has been to improve the convergence not by resorting to a higher order algorithm but simply by locating the nodes in a stategic way so as to condense them in the regions were the curvature is high and to reduce their density in the low curvature regions. It should, however, be noted that this can only be done to a certain extent because when the node density varies too much from one region of the contour to another the loss of accuracy due to the errors introduced in the contour integration in (1.7) adversely affects the convergence in a significant way.

Once the algorithm has converged to a set of values for the positions of the nodes and $V$ (or $\Omega$ ), one would like to find ways of independently verifying that one is in possession of a authentic solution of the Euler equations. We have used in this thesis a number of different approaches to consolidate our belief in the validity of our results. The most direct way has been to compare our results with known ones in parameter limits where the $\mathrm{V}$-state solutions have already been found. For instance, as we will show explicitly later on, the two-layer model solutions often reduce to the Euler ones as the radius of deformation become zero or infinite.

A second way of testing our results has been to measure the linear velocity $V$ (or the angular velocity $\Omega$ ) directly from an initial value problem for which an unperturbed $V$-state is taken to be the initial condition, and to insure that the measured value of $V$ compares well with the value obtained via the relaxation algorithm described above. In practice however, because of the inherent difficulty in measuring $V$ (or $\Omega$ ) with more that two significant figures of accuracy, this method must be considered only as a somewhat gross validation of our solutions.

A last way way of confirming our results is related to the so called "desingularization conjecture" (Zabusky 1981b) which states that if a stationary (i.e. rotating or translating) array of point vortices exists then it should be possible to find an array of $\mathrm{V}$-states of identical circulation with a similar geometry. Conversely, we have tested our algorithm by comparing its solutions, as the areas of the non-zero vorticity regions become very small, with the analytically known point vortex solution of identical circulation. 


\subsubsection{Time-dependent Contour Dynamics}

For studying time dependent processes - e.g. the merger problem, the stability of V-states, and so on - one needs an algorithm that, given a set of contours and the values of the vorticity gradients across them, will allow the calculation of the subsequent evolution of the flow.

The first contour dynamics algorithm was proposed about ten years ago by Zabusky, Hughes and Roberts (1979) as a generalization of the "water-bag" model used to study the Vlasov equations of plasma dynamics (Berk and Roberts 1970, Potter 1976). The main innovation of this technique, as we have already pointed out, is that the velocity at any point in the fluid can be calculated by performing a contour integral around regions of constant vorticity as given by (1.7).

In particular the velocity on the contours themselves can be evaluated in such a way, the contours having been discretized with a finite set of $M_{j}(n)$ nodes located at $\left\{x_{j}^{(n)}(m), y_{j}^{(n)}(m)\right\}$ - the coordinates of the mth node on the $n$th contour in the jth layer. The nodes move according to the equations:

$$
\begin{aligned}
& \frac{d}{d x} x_{j}^{(n)}(m)=u_{i}\left(x_{j}^{(n)}(m), y_{j}^{(n)}(m)\right) \\
& \frac{d}{d x} y_{j}^{(n)}(m)=v_{i}\left(x_{j}^{(n)}(m), y_{j}^{(n)}(m)\right)
\end{aligned}
$$

where the velocities are calculated using the sums in (1.8).

In this thesis we have used three different schemes to perform the discrete time steps necessary to advance the nodes. For maximum accuracy we have relied on a fourth order Runge-Kutta method; this, however, necessitates four "pseudo time steps" to be calculated for each actual time step. When the number of nodes surpasses a few dozens such a calculation becomes prohibitively long for most small computers.

The most time consuming part of the calculation is the evaluation of the velocity at each node, and we have thus given preference to schemes that necessitate the minimal number of velocity evaluations per time step. Of the second order schemes we have used, the fastest is an Adams-Bashforth explicit multi-step method (originally used by Helfrich and Send, 1988) which requires a single evaluation of the velocities at each time step. We have also used a second 
order Runge-Kutta scheme that requires two calculations of the velocities for each time step and is thus quite a bit slower.

Expressions (1.15) reveal clearly the Lagrangian character of a contour dynamical calculation. By this we mean that the nodes themselves behave as tracer particles and are advected by the flow. Of particular interest is the motion of the nodes along the contour which they are supposed to discretize. Originally they are placed at chosen regular intervals, but, as time evolves and contours deform, the nodes tend to condense on one side of the regions of high curvature while the regions of low curvature become depleted. As the nodes move around the contours, the regions of high curvature behave as obstacles that the nodes have great difficulty overtaking (this is connected with the presence of a stagnation point in the comoving streamfunction as will be shown in detail in Chapter 4).

The consequence of this is that, unless some action is taken, as soon as the contours start to develop filaments the calculation has to be stopped because the unevenness in node distribution along the contour produces large errors in the evaluation of the velocities via (1.8). Node redistribution becomes imperative if one wants to be able to continue the computation beyond the very early times. We have used in this thesis two quite different algorithms for node adjustment that we briefly describe next. They are both, however, quite involved and since a thorough discussion is beyond the scope of this thesis, we will limit ourselves here a brief description.

Most node redistribution schemes used until now have followed the one of Overman and Zabusky (1982a and b), the main idea being that node adjustment must be subjected to both local and global constraints. Locally the nodes should be placed so that their density is proportional to some power of the curvature; at the same time the distance between any two nodes must be bounded above and below by a minimum and a maximum value that are independent of curvature. $t$

We have used, in some of our calculations, an algorithm developed by Overman which essentially implements the above two criteria. Every few time steps, the contour is fitted with cubic splines and the nodes are placed such that their density is proportional to the curvature raised to the $1 / 3$ power. This value has been shown to be optimal for the Kirchhoff ellipse (Zou et al. 1987), although other studies have used a $2 / 3$ power density (Dritschel 1988) and have found it to be optimal.

† It is necessary, for instance, to avoid leaving large gaps in regions of low curvature. 
We have also used, for faster though less accurate runs, a crude node insertion and deletion scheme (Meacham et al. 1988). Instead of actually relocating all the nodes along the contour to make their density proportional to some power of curvature, this scheme simply contents itself with measuring the distance between each pair of adjacent nodes and a) if this distance is below some threshold a node is removed or b) if the internodal distance is above some threshold a new node in inserted. In practice the scheme is somewhat more sophisticated because, in order to determine whether nodes need to be added or removed, it makes use of a quantity involving both the distance and some power of the local curvature, so that the criterion for inserting or deleting nodes incorporates curvature information as well and allows greater density of nodes where the curvarure is higher.

However accurate, the node adjustment technique one uses takes nothing away from the fact that enstrophy cascade towards smaller scales is an intrinsic property of two-dimensional vortex dynamics. This implies that, although the area enclosed by the contours remains constant (to within computational errors), the perimeter tends to increase drastically as vorticity is sheared into ever thinner and longer filaments. Thus, if the initial accuracy is to be maintained, the number of nodes must constantly increase, and this simple fact imposes a practical constraint on how far in time the computation can be extended.

The ubiquitous generation of small-scale features with extremely high curvatures and ever increasing perimeter is the principal reason why contour dynamics (even with a good node redistribution scheme) is unable to proceed beyond the first stages in the nonlinear development of unsteady flows. To acceed to the next stage of evolution Dritschel recently proposed a new algorithm called "contour surgery" (Dritschel 1988). The main innovation of contour surgery resides in its ability to perform automatic topological reconnections of the contours. Thus, for instance, a filament can actually be "pinched off" a larger region of vorticity, in which case from a unique contour one obtains two disconnected contours.

Although contour surgery unquestionably represents the state-of-the-art algorithm, its necessity is less than obvious for the kind of questions that we have been interested in addressing in this thesis. The merger problem is a good example: if all one wants to determine is whether two regions of vorticity merge or don't, a short time calculation is sufficient to provide the answer, and the extremely complicated evolution that follows the merger will not shed any further light on the question. 
Sometimes surgery is not necessary to infer the intermediate or even long time behavior of the contours. Thus, for instance, the work of Helfrich and Send (1988) shows that an unstable perturbation of mode $\mathrm{m}$ on a circular baroclinic vortex will lead to the breakup of the vortex into $m$ dipoles that will propagate away from each other. Although they halted the computation before the dipoles had actually completely separated, there was no doubt left as to the long time evolution of the vorticity. The point we wish to make is that, often, a short time calculation is sufficient to understand the overall behavior of the larger structures at long times.

Moreover, at this time, it is totally unknown what types of errrors are introduced by the topological reconnections of contour surgery. As far as the deletion of very small features of vorticity is concerned, one might suppose that its effect may not be dissimilar to a kind of dissipation. However the technique is so new that no in depth quantitative studies have yet been made. Finally, the contour surgery algorithm is cumbersome enough that a supercomputer is absolutely necessary to perform runs in any reasonable amount of time. Thus, in some sense, it destroys the purpose of using contour dynamics as a means of treating fully nonlinear problems with modest computational means.

In general throughout this thesis we have only performed surgery where absolutely necessary. In Chapter 2 we have performed manual (as opposed to automatic) surgery on certain runs, a single time per run, and have repeated the runs, performing surgery at different times, to insure that it did not affect the evolution in any significant way. In Chapter 3 we have used a very simple contour surgery code of Meacham et al. (1988) that has automatic surgery. However, only minimal amounts of surgery were performed before the runs were stopped.

After this long discussion on node redistribution and topological reconnection the reader may have been left with somewhat mixed feelings as to the reliability of the numerical results that we are going to present. However we would like to provide some reassurance by pointing out that, no matter how sophisticated (or unsophisticated!) the algorithm one employs is, the validity of the numerical simulation can be established by a careful monitoring of the conserved quantities of the system.

In particular, the area enclosed by the contours should be conserved exactly since it represents the circulation that, in inviscid and unforced situations such as those we are considering, can be neither created nor destroyed. In all of the computations presented here, with maybe one or two exceptions, the circulation is conserved to within one or two percent in the worst cases 
when surgery has been used. Most of the time, when surgery is not employed, the loss in circulation is of the order of parts per thousand or per ten thousand.

The higher moments of vorticity are also conserved and we have usually monitored the first three (the area and the two first-order moments). Since the changes in the values of the moments that we are interested in detecting are most often extremely small, we have had to design high order algorithms to calculate the moments. We have found the usual second order algorithms to be of insufficient accuracy and have written fourth order ones, which are described in detail in Appendix A.

By a careful use of these diagnostic quantities we have been able to gain much confidence in the reliability of our numerical results. We note that this has proven especially useful in situations, as for instance the runs in Chapter 4, where the qualitative conclusions depend crucially on accurate numerical results.

In conclusion we can say that, for the type of problems of interest in this thesis, only short to intermediate time calculations are necessary and, because we have used a minimal amount of surgery and very careful diagnostics we believe our calculations to be reliable. The questions concerning the accuracy, dependability and interpretation of long time calculations with large amounts of surgery (such as the ones performed by Dritschel, 1988, 1988a and 1988b) are not really relevant to the results presented here. 


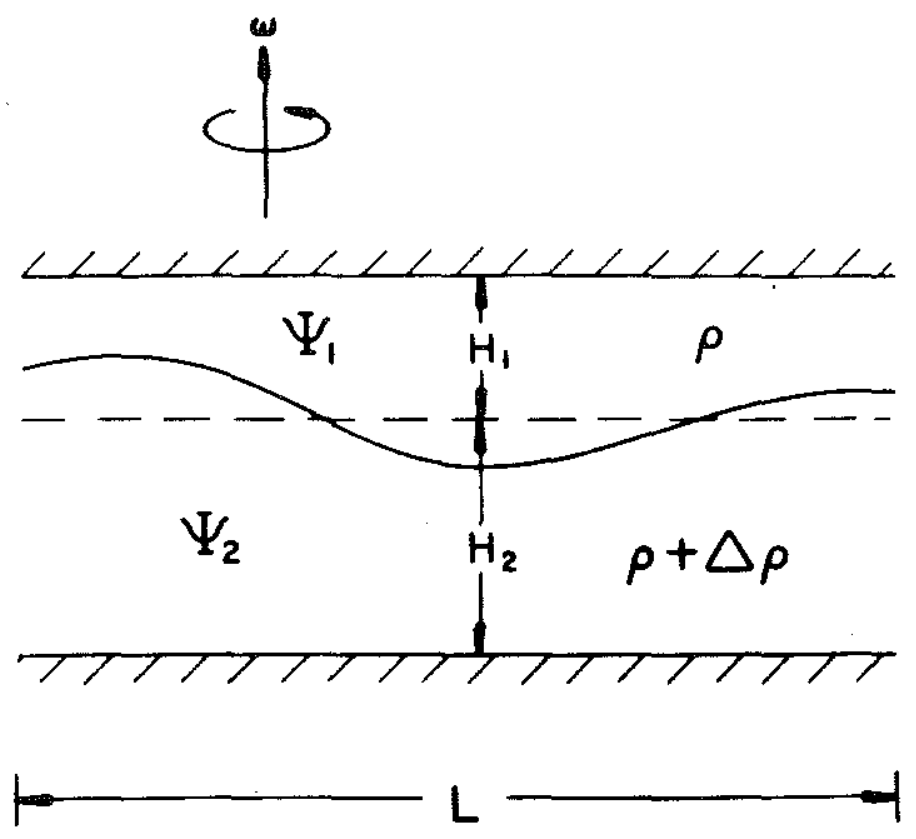

FIGURE 1.1. A schematic drawing of the quasigeostrophic two-layer model. 
(a) 1.0
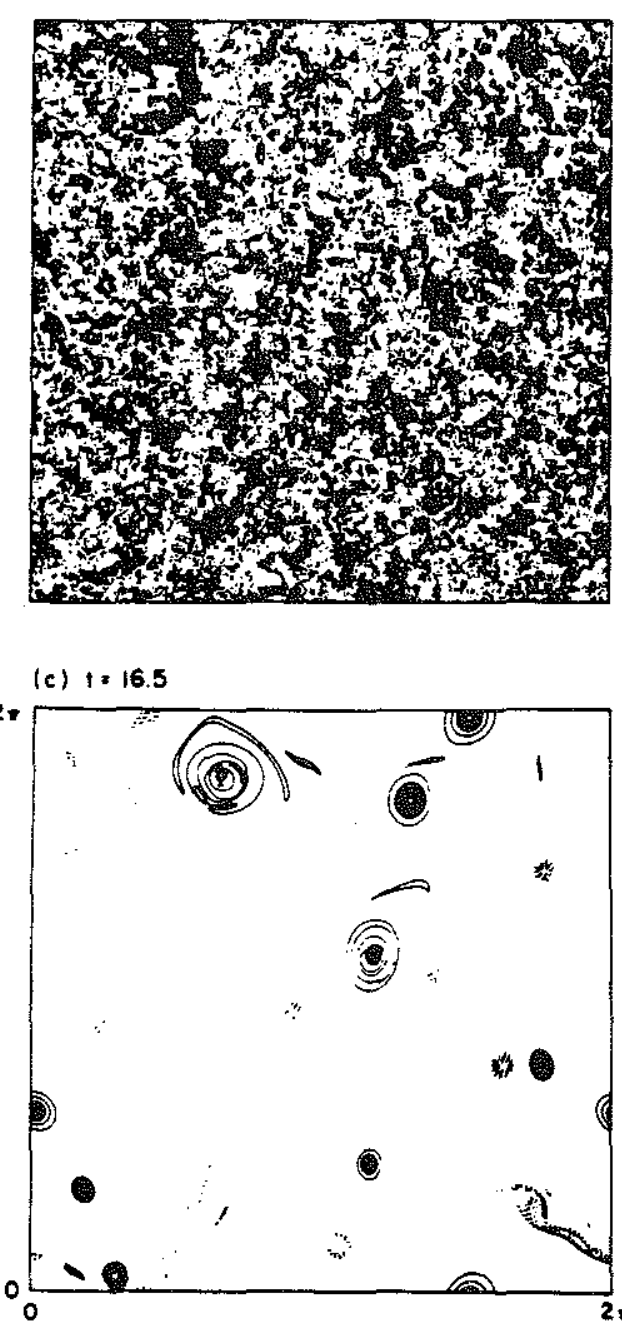

(b) $+=2.5$

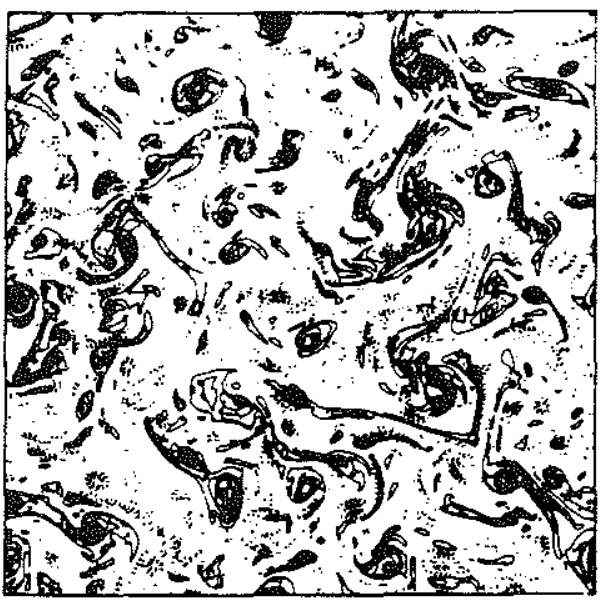

(d) $1 \cdot 37.0$

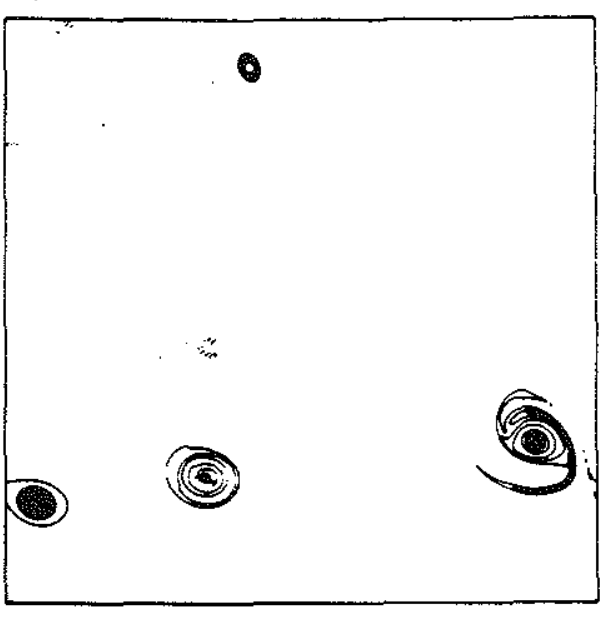

FIGURE 1.2. Emergence of isolated vortical structures from a $k^{-3}$ energy spectrum and random phases at $t=0$. Note that at $t=0$ the vorticity appears to be distributed uniformly, i.e. with no apparent coherent center of vorticity. By $t=2.5$, vorticity regions have begun to condense into vortex centers that can be individually tracked. (From McWilliams 1984). 

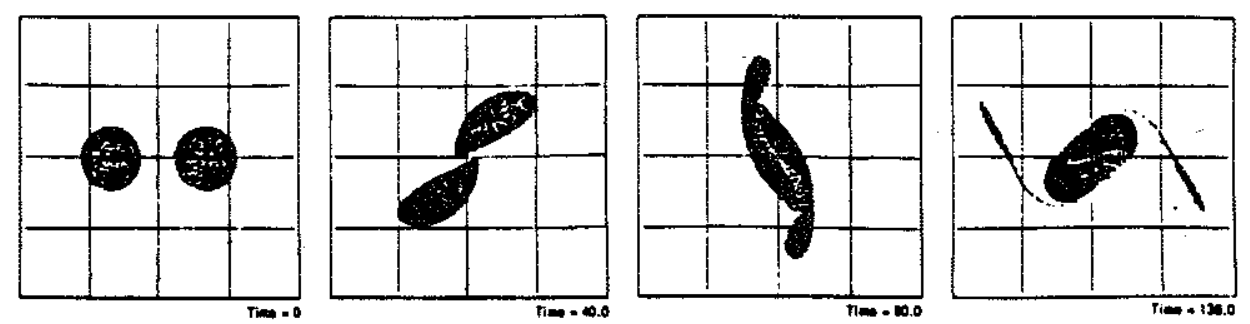

(a)

Two vortices coolescing bocemsen of enticient initial proximity
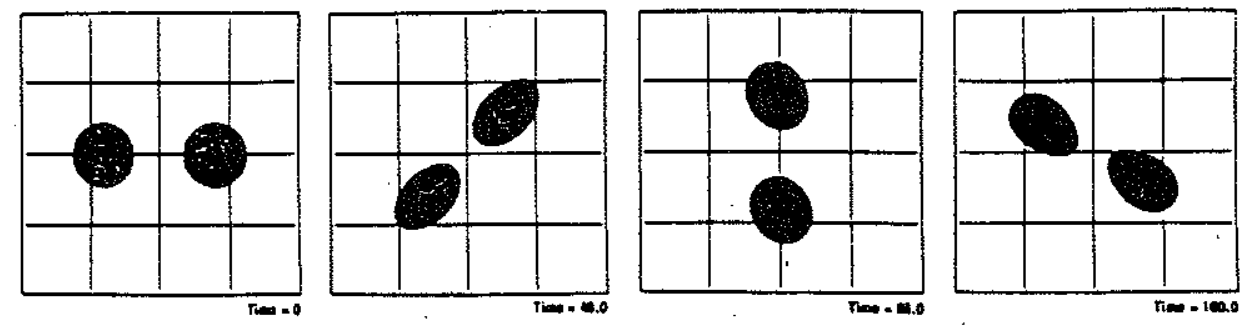

(b)

Two vortices preceasing around esch other. Lerge omplitwo oscilletions on thaif surfectes

FIGURE 1.3. The phenomenology of the merger problem. (From Roberts and Christiansen 1972). 


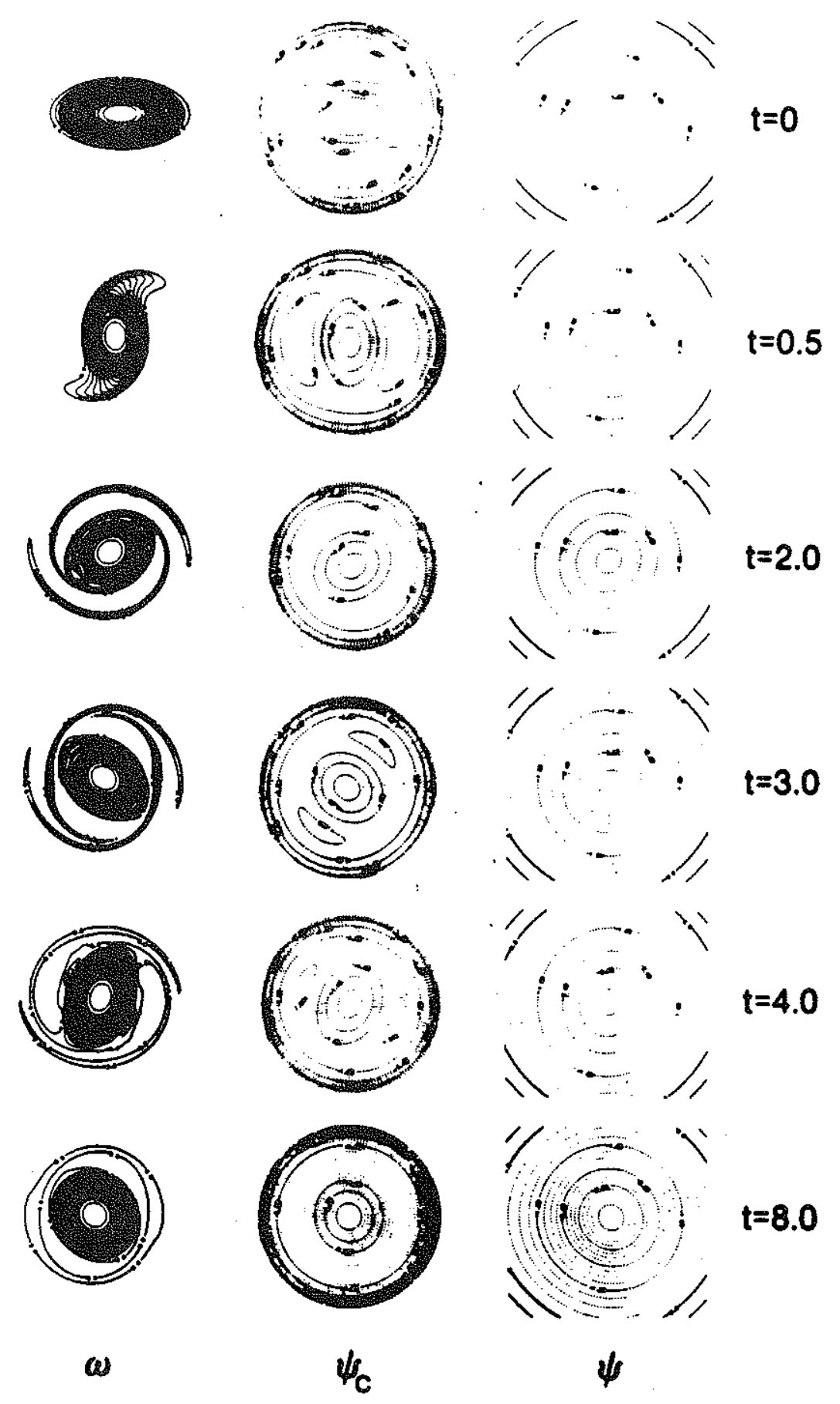

FIGURE 1.4. The evolution of an initially elliptical vortex of aspect ratio $2 / 1$. The first column shows the vorticity $\omega$, the second the instantaneous corotating streamfunction $\psi_{\mathrm{c}}$ and the third the streamfunction $\psi$ in the inertial frame. (From Melander et al. 1987a). 


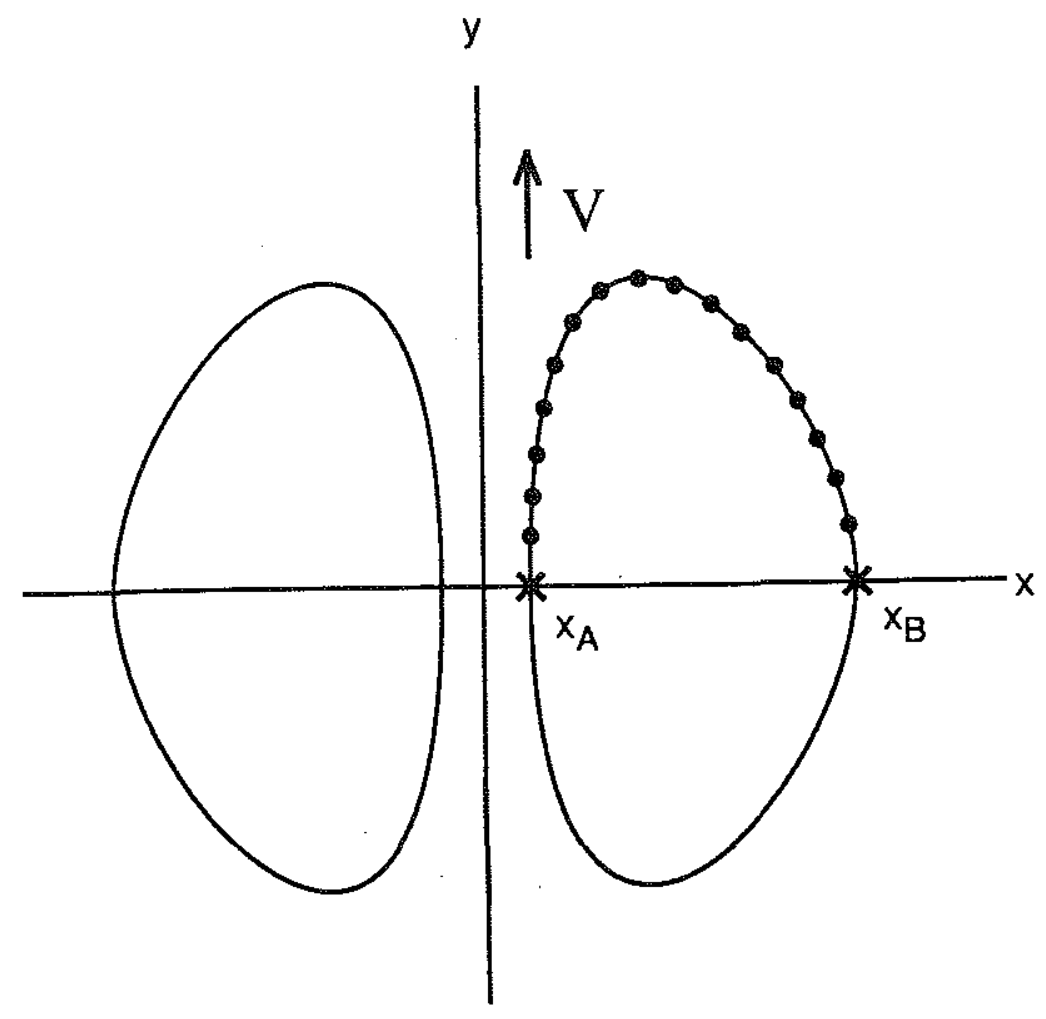

FIGURE 1.5. Schematic drawing of a translating $V$-state. The dots indicate the location of the nodes. The crosses designate the endpoints $x_{A}$ and $x_{B}$, which are fixed during the entire iteration. By symmetry, only the upper right-hand quadrant needs to be computed. 


\section{Two-Layer Dynamics with $\Pi_{2}=0$}

There is no subject so old that

something new cannot be said about it.

Dostoevski

\subsection{Introduction}

As we have already pointed out, one of the objectives of this thesis has been to investigate the vortex dynamics of the two-layer model using our knowledge of two-dimensional vortex dynamics as a starting point, and progressively increasing the complexity of the system towards a stratified quasi-geostrophic configuration. We have taken the first step away from exact two-dimensionality by considering the effect of the presence of a finite layer of zero geostrophic potential vorticity that is coupled to a two-dimensional fluid.

We thus limit our attention in this chapter to situations in which the lower layer has no geostrophic potential vorticity, i.e. such that $\Pi_{2}(x, y)=0 t$. It is important to keep in mind, however, that although the geostrophic potential vorticity vanishes exactly neither the velocity nor the relative vorticity fields are zero in that layer. In other words, the lower layer participates actively in the dynamics of the upper layer, even if $\Pi_{2}$ vanishes.

For purposes of solving the two-layer model equations (1.1) to study the upper-layer dynamics it is, however, unnecessary to calculate the lower-layer fields when a contour dynamical approach is used. Indeed, as can be seen from (1.6), the evolution of the fields (in both layers) is determined entirely from the positions of the contours delimiting the regions of constant geostrophic potential vorticity in the upper layer alone. In all the calculations of this chapter we have thus confined our attention to the upper layer, where the geostrophic potential vorticity is located.

$\dagger$ To dispel any possible confusion, we remind the reader that $\Pi$ is not the full potential vorticity but only its quasigeostrophic component. Thus $\Pi=0$ does not imply $\zeta=-f$. 
The simplification introduced by setting $\Pi_{2}=0$ implies that, of the four Greens' functions defined in (1.4), only one remains dynamically important, namely $G_{11}$ which represents the effect on the upper layer of vorticity on that layer, and is given by:

$$
G_{11}(r)=\frac{\delta}{1+\delta} \log r-\frac{1}{1+\delta} K_{0}(\Gamma r)
$$

where

$$
\Gamma=\gamma(1+\delta)^{1 / 2}
$$

and where, we recall, $\delta$ is the ratio of the layer thicknesses at rest and $\gamma$, the inverse of the radius of deformation, quantifies the degree of coupling of the two layers. Therefore, as far as the upper layer is concerned, the effect of the presence of an active lower layer with $\Pi_{2}=0$ manifests itself through the fact that the purely logarithmic Greens' function of the 2D Euler equations is replaced by $G_{11}$.

Note, from the form of $\mathrm{G}_{11}$, that the vorticity can interact in two distinct ways. The logarithmic term corresponds to the barotropic mode of interaction of the Euler equations, with the difference that, in (2.1), it is reduced by a factor of $\delta /(1+\delta)$. As the thickness of the lower layer vanishes, i.e. $\delta \rightarrow \infty$, the dynamics of the upper layer becomes dominated by this logarithmic term and reduces identically to twodimensional Euler dynamics.

At the other extreme, as $\delta \rightarrow 0$, i.e. as the lower layer becomes infinitely deep, we recover the Greens' function $-K_{0}(\gamma r)$ of the so called "equivalent barotropic" modelt. This modified Bessel component of $G_{11}$ represents the baroclinic mode of interaction of the vorticity in the upper layer, and, of course, it tends to vanish as the lower layer thickness becomes very small. It is this component of the Greens' function that is responsible for the new dynamics introduced by the presence of an active lower layer.

In order to investigate this new dynamics we have, in analogy with what has been done for the Euler equation, concentrated our efforts on two particular questions: the stationary configurations of vorticity (the V-states) and the merger problem. Usually, in addition to $\gamma$ and $\delta$, there is at least one other parameter that can be chosen so that, in general, the parameter spaces that need to be investigated are, at least, three dimensional. To restrict the amount of computations to tractable dimensions while, at the same time, capturing all of the principal

† Sometimes also referred to as the "reduced gravity" model. 
qualitative behaviors that are found in the different regions of the parameter space, we have proceded as follows in our selection of the values of $\delta$ and $\gamma$.

We have decided to limit ourselves to three interesting and representative values of $\delta$ : the equivalent barotropic model $(\delta=0)$, the case of two equal layers $(\delta=1)$ and, as an intermediate case, the value $\delta=.2$, which roughly corresponds to the ratio of the depth of the oceanic thermocline to the full depth of the ocean. Computational evidence will be presented in this chapter that at large values of $\delta$ i.e. $\delta>1 !-$ the vortex dynamics in the upper layer are essentially identical to twodimensional Euler dynamics.

The parameter $\gamma$ represents the strength of the coupling between the two layers, and the approach that we have adopted throughout this thesis has been to investigate how the dynamics of the two layer model changes as this coupling between the layers is progressively increased from zero to infinity.

For the simplified situation of interest in this chapter, i.e. $\Pi_{2}=0$, in the weak coupling limit it is easy to show that, due to the logarithmic behavior of the $\mathrm{K}_{0}$ function at small values of the arguments,

$$
\lim _{\gamma \rightarrow 0} G_{11}(r)=\log r
$$

for all values of $\delta$. This simply states that, as the coupling is reduced to zero, the dynamics in the upper layer becomes exactly two-dimensional. The more interesting limit is the one for large values of $\gamma$, when the coupling between the layers becomes very strong.

For that case, using the asymptotic approximation for the modified Bessel function at large arguments, we can write

$$
G_{11}(r) \approx \frac{\delta}{1+\delta} \log r-\frac{1}{1+\delta}\left[\frac{\pi}{2 \Gamma r}\right]^{1 / 2} e^{-\Gamma r} \text { for } \quad \gamma>>1
$$

The case of interest, as we have already mentioned, is when $\delta$ is small, and here great care must be used in taking the limit because of the competition of two small terms in (2.3). As long as $\delta$ is different from zero, the exponential term will eventually become smaller than the logarithmic one, no matter how small $\delta$ is.

\footnotetext{
† We must point out that the arguments presented next would lose their validity were the fields at each point determined mostly by the contributions from the vorticity lying at $r<1$, in which case (2.3) would be invalid even in the limit $\Gamma \gg 1$. It turns out that such is not the case, and that the arguments derived here are strongly confirmed by the numerical results of the following sections.
} 
Therefore we can write:

$$
\lim _{\gamma \rightarrow \infty} G_{11}(r)=\frac{\delta}{1+\delta} \log r \quad \text { for } \quad \delta \neq 0
$$

If, however, we chose $\delta=0$ in (2.1), i.e. we restrict our attention to the equivalent barotropic model from the start, then the coefficient of the logarithmic term in (2.3) is identically zero, and, in the strong coupling limit, the Greens' function behaves as a decaying exponential, namely

$$
\lim _{\gamma \rightarrow \infty} G_{11}(r)=\left[\frac{\pi}{2 \gamma r}\right]^{1 / 2} e^{-\gamma r} \quad \text { for } \quad \delta=0
$$

From this it is easy to conclude that at large $\gamma$, i.e. at scales that are large compared to the deformation radius, the equivalent barotropic model can be expected to behave in a drastically different way from the Euler equations. The Greens' function (2.5) is characteristic of shielded vortices, whose interaction is extremely local and is therefore fundamentally different from the very long-range logarithmic interaction characteristic of two-dimensional Euler dynamics.

The equivalent barotropic model is often used as a simple shortcut to a full baroclinic calculation, whereby one retains the simplicity of single-layer dynamics and, at the same time, tries to capture the effect of an underlying lower layer. The implication of (2.3) is that, when the radius of deformation becomes small, the equivalent barotropic model simulates a two-layer calculation for which $\delta<\delta_{\mathrm{eb}}$, where $\delta_{\mathrm{eb}}$ is approximately given by:

$$
\delta_{e b}=O\left(\gamma^{-1 / 2} e^{-\gamma}\right)
$$

It turns out that even at relatively innocuous values of $\gamma$ the value of $\delta_{e b}$ is extremely small due to its exponential dependence on $\gamma$. For instance, at scales only three times larger than the radius of deformation $(\gamma=3)$ the ratio of upper to lower layer depths simulated by the equivalent barotropic model is approximately $1 / 35$, and at $\gamma=5$ (not what one would call an asymptotic regime!) the value of $\delta_{\mathrm{eb}}$ is of the order of $1 / 330$.

In summary, the point to be retained is the following: for motions on scales a few times the radius of deformation or larger, the equivalent barotropic model will generate dynamics that are extremely different from those a model with two finite layers. Therefore, on those scales, a full two-layer calculation is necessary and the 
equivalent barotropic shortcut should be avoided. This conclusion, which will be confirmed by the variety of numerical results presented in this chapter, is probably one of the most important results to emerge from this study and suggests that the results previously obtained with equivalent barotropic models may have to be reexamined.

\subsection{Upper-layer V-states}

After having obtained, from a careful consideration of the asymptotic forms of the Greens' function, a first indication of the behavior that is to expected in the various limiting cases, we now proceed to present our results for the stationary vorticity configurations in the upper layer. As was reviewed in the previous chapter, a great number of $\mathrm{V}$-states have been determined for the two-dimensional Euler equations, but it would certainly take more than a single thesis to find all of their two-layer analogs.

We have thus decided to confine our results to the three simplest and most interesting types of $V$-states: the $m=2$ rotating $V$-states (generalizations of the Kirchhoff ellipse to the $\mathrm{G}_{11}$ Greens' function), the same-layer translating V-states (dipoles with both positive and negative vorticity in the same layer) and the doubly connected corotating $V$-states (first determined for the Euler equations by Saffman and Szeto 1980). The latter are intimately connected with the merger problem and we have therefore delayed their discussion to the last section of this chapter (section 2.4) after the merger problem will have been discussed in depth.

\subsubsection{Rotating $(m=2) \quad$ V-States ${ }^{\dagger}$}

We start with the simplest possible vorticity configuration in a two-layer context (illustrated schematically in Figure 2.1): a patch of constant vorticity in the upper layer rotating without change in shape at constant angular velocity $\Omega$. Because of symmetry, it is sufficient to determine only one quarter of the shape for any value of the two endpoints, designated by $R_{A}$ and $R_{A}$. Of these two lengths only

The value of $m$ represents the wavenumber of the perturbation on a circle of which these solutions are the finite amplitude extensions. Solutions up to $m=6$ have been determined by Wu et al. (1984) for the Euler equations. 
one has to be varied and, without loss of generality, we have chosen the aspect ratio $\lambda \equiv R_{A} / R_{B}$ as the parameter of interest. The numerical details of the algorithm are given in Appendix B1.

Since for each value of the parameters $\lambda, \delta$ and $\gamma$ a V-state can be determined; the parameter space is three dimensional and, as explained in the previous section, we have carried out its investigation by using only a small but representative set of parameter values. We have selected three values of the aspect ratio $\lambda$ : a slightly elliptical shape $\lambda=3 / 2$, an intermediate value $\lambda=5 / 2$ and a very elongated one $\lambda=7 / 2$. For each of these we have calculated solutions at the three values $\delta=0,0.2$ and 1 as explained above.

To observe the variation in shape as the coupling constant $\gamma$ is increased from zero, we have for each fixed value of $\lambda$ and $\delta$ obtained the $V$-states for 21 values of $\gamma$ that span, in approximately equal intervals (on a logarithmic scale), the three orders of magnitude $10^{-2} \leq \gamma \leq 10^{2}$. The reason for extending our investigation to such high and low values of $\gamma$ is that we want to insure that we cover the asymptotic results known for the limits $\gamma<<1$ and $\gamma>1$.

Recall that, for a purely logarithmic Greens' function, the shape of the boundary is an exact ellipse. Because of the asymptotic behavior of $G_{11}$, we expect, for $\delta \neq 0$, to recover exactly elliptical shapes in both the $\gamma<<1$ and $\gamma>1$ limits. The real questions can then be restated in the following simple form: as $\gamma$ is varied between zero and infinity, how do the $m=2$ rotating $V$-states differ from Kirchhoff ellipses? How does this difference depend on $\delta$ and $\lambda$ ? What happens at $\gamma>1$ for the equivalent barotropic case $\delta=0$ ? The answer to these questions is presented in Figures $2.2,2.3$ and 2.4 for $\lambda=3 / 2,5 / 2$ and $7 / 2$ respectively.

Consider first the case $\lambda=3 / 2$ for which the solutions are shown in Figure 2.2 (for $\delta=0,0.2$ and 1). Only the upper right quadrant is plotted and, for clarity, only the three $V$-states corresponding to $\gamma=0.01,1$ and 10 are shown (the solid lines represent the Kirchhoff) ellipse. It is quite remarkable to observe that, quite independently of $\delta$, the deviations from the Kirchhoff ellipse are extremely small, at this aspect ratio, over the entire range in $\gamma$.

To make a quantitative estimate of the difference between the upper-layer $V$-states and the Kirchhoff ellipse, we have calculated the quantity $\delta A$ defined by:

$$
\delta A=\frac{A_{K}-A}{A_{K}}
$$


where $A_{K}$ and $A$ are the areas of the Kirchhoff ellipse and of the $V$-state respectively. This quantity, which represents the fractional change in area, is shown in Figure 2.2 as a function of $\gamma$ for the three values of $\delta$ for which solutions were computedt.

An inspection of this quantity immediately reveals the qualitatively different behavior of the equivalent barotropic model $(\delta=0)$ at large $\gamma$ that we have argued from an analysis of the Greens' function. We note incidentally, that the $\delta=0$ large- $\gamma$ limit presents computational problems as well. Since the Greens' function decays exponentially, most of the vorticity interaction is extremely local and this affects considerably the convergence of our algorithm. The velocity is essentially zero everywhere in that limit, except for strong jets located around the position of the contours. This strong inhomogeneity in the velocity fields is probably responsible for the increased difficulty in achieving convergence in that limit.

Note, moreover, how small the fractional change in area is when both layers are finite. Even for a lower layer five times as deep as the upper layer, the maximum change in area is of the order of one percent. This number changes to a few percent for the slightly larger aspect ratio $\lambda=5 / 2$, for which the solutions are presented in Figure 2.3. To keep the figures legible, we have chosen to present only a selected number of $V$-states for each value of $\delta$ (the ones for $\gamma=0.01$ and $\gamma=10$ are always shown). The poorer convergence of our algorithm at high $\gamma$ for $\delta=0$ may also be due, in part, to the emergence of negative curvature sections at higher values of the aspect ratio.

This is even more apparent at $\lambda=7 / 2$, as can be seen from Figure 2.4 . The equivalent barotropic solutions at large $\gamma$ bulge away from the Kirchhoff ellipse, and give rise to "peanut" shaped vortices. This effect is undoubtedly the consequence of the exponentially decaying character of the Greens' function, and it is considerably weaker when both layers are finite. In that case, over the entire $\gamma$ range, the solutions are remarkably close to Kirchhoff ellipses, even at this high aspect ratio.

In Figure 2.5 we have plotted the angular velocity $\Omega$ as a function of $\gamma$ for the various values of $\delta$ and $\lambda$ for which the solutions were determined. For non-zero $\delta$, both the large and small $\gamma$ limit are finite and, from (2.2) and (2.4), it is easy to see that they are simply given by:

T The jaggedness of the curves is due to the fact that $V$-states were calculated at discrete values of $\gamma$. 


$$
\lim _{\gamma \rightarrow 0} \Omega=\Omega_{\mathrm{K}} \quad \text { and } \quad \lim _{\gamma \rightarrow \infty} \Omega=\frac{\delta}{1+\delta} \Omega_{\mathrm{K}} \quad \text { for } \delta \neq 0
$$

where

$$
\Omega_{\mathrm{K}}=\frac{\lambda}{(1+\lambda)^{2}}
$$

is the angular velocity of a Kirchhoff ellipse of aspect ratio $\lambda$. Note however that, in the equivalent barotropic case $\delta=0$, the angular velocity $\Omega$ goes to zero in the limit of large $\gamma$. Why this is so can be easily understood by recalling that, in that limit, the Greens' function is an exponential that decays extremely fast, so that, each portion of the vortex is influenced almost uniquely by the closest surrounding vorticity.

We have fitted the $\log$-log curves of $\Omega$ vs $\gamma$ for $\delta=0$ at large values of $\gamma$ with straight lines, and have found the slopes to be equal to $2.94,2.93$ and 2.89 for $\lambda=3 / 2,5 / 2$ and $7 / 2$ respectively. This numerical result is in good agreement with the results of Pratt \& Stern (1986), who, in developing an asymptotic theory for the evolution of a perturbation on a vorticity front in the equivalent barotropic model at large $\gamma$, found that a dominant balance in the vorticity equation could be achieved only when time was scaled by $\gamma^{-3}$.

Their derivation, however, is convoluted enough to obscure the fundamental balance that leads to this scaling, and we have formulated a very simple argument that shows how it arises. Let $R(\theta)$ be the radial distance of the contour from the origin. The dimensional streamfunction $\Psi$ must satisfy the condition:

$$
\frac{\partial}{\partial t} R \approx \frac{1}{R} \frac{\partial}{\partial \theta} \Psi
$$

for all angles $\theta$ on the boundary. The small parameter of interest is $\gamma^{-1}$, and we are considering the case $\gamma>>1$ which corresponds to motions on scales large compared to the radius of deformation (recall that $\gamma$ is defined as the ratio $L / L_{R}$ ). At leading order in $\gamma^{-1}, \Psi$ is independent of $\theta$ and it is therefore the $O\left(\gamma^{-1}\right)$ correction to $\Psi$ which allows the above boundary condition to be satisfied in time. In order to determine the time scale define:

$$
R=L R^{*} \quad \Psi=\left[q L_{R}^{2}\right] \gamma^{-1} \Psi^{*} \quad t=\gamma^{n} q^{-1} t^{*}
$$

where the starred quantities are nondimensional and $O(1)$, and $q$ is the vorticity scale. Note how $\Psi$ has been scaled one order smaller than the leading order 
geostrophic streamfunction. Substituting these scalings in the above boundary condition and requiring that both sides be of the same order yields the value $n=3$. We note that this scale seems to be fundamental to the dynamics of the equivalent barotropic model in the large $\gamma$ regime, since it arises naturally in a number of different contexts. For instance, one can show analyticallyt that the angular velocity of an infinitesimal perturbation on the boundary of a Rankine vortex in the equivalent barotropic model is proportional to $\gamma^{-3}$ when $\gamma>1$.

\subsubsection{Translating V-States (Dipoles) ${ }^{\ddagger}$}

All of the qualitative conclusions that have emerged from the study of the rotating $\mathrm{V}$-states are confirmed by the investigation of the other most important type of coherent vortex structure: the dipole. We consider here a vortex composed of two regions of opposite-signed geostrophic potential vorticity of the same strength in the upper layer. The vortices induce motion on each other in such a way that the compound vortex propagates without change in shape at constant velocity $\mathrm{V}$ along the $y$-axis, as is illustrated schematically in Figure 2.6. For simplicity we limit our attention to vortices in which the two regions are perfectly symmetric upon reflection about the $y$-axis.

Each dipole is characterized by its inner and outer radius, $x_{A}$ and $x_{B}$ respectively (cf. Figure 2.6). Without loss of generality we set $x_{B}=1$ and define $\mu \equiv \mathrm{X}_{\mathrm{A}} / \mathrm{x}_{\mathrm{B}}$ to be the parameter to be varied. As for the rotating $\mathrm{V}$-states we have determined the shapes for a few values of $\mu$ and $\delta$, and have concentrated our attention on the variations as the coupling constant $\gamma$ is increased from zero to very large values. One difference with the rotating $V$-states is that the corresponding Euler solution is not known analytically. The translating $V$-states for the Euler equations, first obtained by Deem and Zabusky (1978a and b) are numerical solutions. The exception to this, as one would have suspected by now, is the case $\delta=0$ (equivalent barotropic model) at $\gamma>>1$. In that limit the Greens' function becomes a very rapidly decaying exponential, and the solution corresponds to two exact circles of opposite vorticity that are stationary (i.e. $V=0$ ).

The major difference between the rotating and translating $\mathrm{V}$-states is that in the latter case, depending on the value of the parameter $\mu$, there exist two

\footnotetext{
$\dagger$ We will do this in Chapter 4.

\# See Appendix B2 for details concerning the algorithm used in this section.
} 
qualitatively different behaviors of the solutions as $\gamma$ is varied (in the previous section we have seen that as $\gamma$ is increased from zero the solutions always "bulge out" from the Kirchhoff ellipse for all aspect ratios $\lambda$ ). We illustrate this by considering two values of $\mu$ in the different regimes.

In Figure 2.7 the results are shown for $\mu=0.2$ (we have used the same values of $\delta$ and $\gamma$ as for the rotating $V$-states). For $\delta \neq 0$ the vortices always have a smaller aspect ratio, at finite values of $\gamma$, than the Deem and Zabusky dipoles. Note that, for $\gamma<<1$ and $\gamma>1$, the Euler solution is recovered, in agreement with (2.2) and (2.4) respectively (i.e with the Euler value of $V$ scaled by a factor $\delta /(1+\delta)$ in the limit $p>1$, according to 2.4). The equivalent barotropic solutions behave differently, and the shapes progressively relax to circles as $\gamma \rightarrow \infty$. To quantify the results we have plotted the ratio $A / A_{C}$ versus $\gamma$, where $A$ is the area of one of the vortices of the $V$ state and $A_{C}$ the area of the circle of radius $\left(x_{B}-x_{A}\right) / 2$.

When the ratio $\mu$ of the inner to the outer radius becomes less than a critical value $\tilde{\mu}$ (we have found $\tilde{\mu} \approx 0.09$ ), the behavior, as $\gamma$ is varied, become qualitatively different. As $\gamma$ is increased from zero the $V$-states become more elongated than the Euler ones. We present the results for $\mu=0.06$ in Figure 2.8. Note that for the equivalent barotropic case the maximum deformation, which occurs for $\gamma \approx 7$, is very large (the area essentially doubles), while for the cases in which $\delta \neq 0$ the change in area compared to the Euler shapes is of only a few percent, as was the case for the rotating solutions.

The velocity of propagation of the dipole is plotted as a function in $\gamma$ in Figure 2.9. At $\gamma=0$ the Euler value is recovered, as well as in the limit $\gamma>1$ scaled by the factor $\delta /(1+\delta)$ in accordance with (2.4). For the equivalent barotropic case the velocity vanishes as $\gamma \rightarrow \infty$, but we have been unable to fit the $\log -\log$ plot of $V$ vs $\gamma$ with a straight line.

The two main conclusions that had been drawn from the analysis of the rotating $\mathrm{V}$-states have been strengthened by the investigation of the translating ones. To summarize, we have found, on one side, a great insensitivity of the dynamics in the upper layer to the presence of the lower layer for $\delta \neq 0$ (and its similarity to Euler dynamics) and, on the other side, the anomalous behavior of the equivalent barotropic model $(\delta=0)$ when the coupling of the two layers becomes very strong $(\gamma>1)$. These conclusions, obtained by the study of static vorticity configurations, will now be tested with time-varying vorticity fields as we proceed to consider the question of merger. 


\subsection{Merger of Vorticity in the Same Layer}

Since the early numerical studies of two-dimensional vortex dynamics, the problem of merger of two structures of equal-signed vorticity has occupied a favorite place (Roberts and Christiansen, 1972 and Christiansen and Zabusky, 1973). As we pointed out in the previous chapter, the importance of this fundamental process resides in its being the principal mechanism through which the energy cascade to large scales is enacted. The most important result for initially-circular constant-vorticity two-dimensional vortices is that merger will occur if the ratio of the centroid distance to the radius at the initial time is smaller than (approximately) 3.3.

It is important to keep in mind that the merger boundary is somewhat "fuzzy", at the level of accuracy to which we have decided to confine ourselves in this chapter, and the decision as to whether merger did or did not occur can be a subjective one. Near the critical initial ratio one often observes one or more filaments of vorticity being exchanged between the two vortices which, however, retain their identity and do not coalesce into a single structure. Thus the transition from no-merger to merger is a smooth one. Although one can define an objective criterion for deciding whether merger has occurred (as we have done in the next chapter), we are in this section mostly interested in a zeroth order investigation of the merger question in the two-layer model.

The presence of two layers of fluid immediately presents an additional degree of freedom to the merger problem, in as much as we can consider the two vortices in the same or in different layers. We have distinguished between these two processes by assigning to them different names. In the context of a quasigeostrophic system composed of two (or perhaps several) layers we designate by merger the process by which two vortices in the same layer coalesce into a single one. The interaction of regions of equal-signed vorticity residing in different layers will be referred to as the alignment problem.

Since this chapter is dedicated to the study of situations in which all of the vorticity resides in the upper layer, we have deferred the study of the alignment problem to the next chapter. The question to be addressed here can then be posed in the following very concise form: how much does the Euler value 3.3 change when the logarithmic Greens' function is replaced by $\mathrm{G}_{11}$ ? how far from 3.3 does 
the critical merger distance deviate as a function of $\delta$ and $\gamma$, the two parameters of the two-layer model?

\subsubsection{Review of Recent Laboratory Experiments}

In a recent series of papers Griffiths and Hopfinger $(1986,1987)$ have addressed this very question by performing laboratory experiments with two fluid layers of equal depth (i.e. at $\delta=1$, in our notation). They specifically constructed their laboratory setup in such a way as to be able to simulate a two-layer system obeying the quasigeostrophic equations (1.1).

In order to confirm the quasigeostrophic nature of the finite-area vortices that they were able to generate in their experiments, Griffiths and Hopfinger very carefully compared the velocity field of their circular vortices as a function of the radial distance from the center against the theoretical results obtained from the solution of $(1.1 \mathrm{~b})$ for a circular distribution of vorticity. They found the agreement between the experimental data and the quasigeostrophic theory to be good, and presented evidence to consolidate their belief that their laboratory setup was indeed obeying two-layer geostrophic dynamics.

Their results for the the dependence of the critical merger distance on the inverse radius of deformation $\gamma$, that we have reproduced in Figure 2.10 (adapting the data of Figure 10 from Griffiths and Hopfinger 1987 to our notation) is however surprising since, although the authors seem to have been unaware of the fact, it is at odds with what one would expect to observe for a system that obeys the quasigeostrophic two-layer dynamics described by (1.1). Even more disturbing is the fact that this discrepancy occurs in the weak coupling regime (i.e. $\gamma<<1$ ), in which, as we have already shownt, the dynamics in the upper layer should reduce to simple Euler dynamics.

More specifically, concerning Figure 2.10, it is difficult to understand how, in the limit of weak coupling $\left(\gamma^{-1}>1\right)$ the critical merger distance could assume a value so different from the Euler value of 3.3. Notice that in the more delicate limit of strong coupling, which corresponds to $\gamma^{-1}<<1$, the Euler value is recovered quite accurately, in agreement with the result of (2.4). A number of effects could be mentioned as possibly responsible for the remakable difference between the

T See equation (2.2) and the discussion thereabout. 
results of Griffiths and Hopfinger and the predictions of quasigeostrophic therory, but, mostly because we have had no access to how the experiment was performed beyond what has appeared in print, we have been unable to arrive at a convincing argument that would allow one of them to emerge as the certain cause.

We believe nonetheless that excessive friction can be excluded as a possible cause for the discrepancy because, had it been present without the knowledge of the experimenters, it would have affected the results at $\gamma^{-1} \ll 1$ even more dramatically then at $\gamma^{-1}>>1$, since the times for merger are a lot longer in the large $\gamma$ regime (typically one order of magnitude, as reported by Griffiths and Hopfinger 1987). Moreover it is quite implausible that the discrepancy betwen these experiments and the predictions of quasigeostrophic theory be due to friction since both merging vortices are placed in the upper layer and therefore dissipation has to act via the density interface. Finally, Griffiths and Hopfinger (1987) report that they measured the dissipation time scale in their experiment to be of the order of more than 20 rotation periods $T_{\Omega}$, while most mergers for $\gamma^{-1}>2$ took place in less than $5 \mathrm{~T}_{\Omega}$.

What we suggest might have been playing a role unsuspected by the experimenters is the presence of a particularly strong Eddington-Sweet circulation in the $\gamma^{-1} \gg 1$ range. It is well known that a stratified fluid cannot sustain exact solidbody rotation, and that, after spin-up, a residual radial circulation is produced by the density gradient, and the strenght of the circulation increases proportionally to this gradient. Because the $\gamma^{-1}>>1$ regime corresponds to $L_{R}>>1$, which in turn corresponds to $\Delta \rho / \rho>>1$, it is not impossible that an Eddington-Sweet circulation considerable enough to affect the results might have been present in the experiments of Griffiths and Hopfinger (1987).

In the final analysis, however, we recognize that, not having access to the experimental apparatus used and not being familiar with the way in which the experiment was conducted, we can only speculate as to why Griffith and Hopfinger's results are what they are. There is however little doubt that they are incompatible with vortex dynamics in the nearly inviscid quasi-geostrophic finitearea constant potential vorticity approximation. Further evidence in support of this assertion will be provided by the results presented in this and the next section. 


\subsubsection{Numerical Results for the Merger Problem}

We have conducted three sets of initial value numerical experiments to explore the dependence of the critical merger distance on the two parameters of the quasi-geostrophic two-layer model. As for the $\mathrm{V}$-states we have limited our attention to the three "canonical" values of $\delta(0,0.2$ and 1) and for each of these we have covered the whole range of interest in $\gamma$. The initial condition is taken to be the following: two circular regions of constant potential vorticity and radius 1 located (in the upper layer) on the $x$-axis and separated by a distance $d_{c}$.

The first set of results, for layers of equal depths, is shown in Figure 2.10 where we have drawn a cross at the values of $d_{c}$ and $\gamma$ where merger occurred and a circle otherwise. After having seen the results for the V-states it is not surprising to observe the great insensitivity of the results to changes in the coupling constant $\gamma$. We have drawn in by hand, in Figure 2.10, a curve labelled $d_{\text {critical }}$ whose only purpose is to separate the crosses from the circles to enhance the clarity of the results. From the fact that we have drawn a flat curve it should not be concluded that $d_{\text {critical }}$ is independent of $\gamma$, but rather that no dependence could be detected at the resolution, in the $\left(\mathrm{d}_{\mathrm{c}}, \gamma\right)$ plane, with which these experiments were conductedt .

In Figure 2.11 we show the results for the case $\delta=0.2$. As the lower layer is made deeper, its influence on the dynamics of the upper layer becomes more noticeable. In particular we note that the qualitative shape of the curve (with the exception of the perplexing $\gamma^{-1}>1$ range) is in accordance with the results of Griffiths and Hopfinger. Specifically, we refer to the fact that, as the presence of the active lower layer is felt by the vorticity in the upper layer, the merger distance decreases, i.e. two vortices need to be placed closer to each other before they can merge. This is, of course, the result of the baroclinic mode of interaction ( via the $\mathrm{K}_{0}$ Greens' function) that plays a detectable role at $\gamma \approx 1$. As expected, the 3.3 value in both the large and small $\gamma$ limit is recovered.

A final set of runs was made for the equivalent barotropic model $(\delta=0)$, and the results are shown in Figure 2.12. Again the $\gg>1$ limit exhibits singular behavior and the Euler value 3.3 for the critical merger distance is not recovered as $\gamma^{-1} \rightarrow 0$. This behavior is easily understood from the consideration of (2.5): at $r>1$

\footnotetext{
† Computational evidence will be given, in Section 2.4 , that indicates a very weak depence on $\gamma$ at $\delta=1$.
} 
the shielding due to the asymptotically exponential behavior of the $\mathrm{K}_{0}$ function is so strong that two vortices have to be placed extremely close before they can merge.

\subsubsection{Non-axisymmetrization in the equivalent barotropic model at $\gg>1$}

In the previous section we have merely been interested in determining whether or not merger will occur for a particular initial condition characterized by the value of the two parameters $d_{c}$ and $\gamma$. In this section wish to take a closer look at the merger events themselves, and try establish how they differ from Euler mergers due to the presence of the active lower layer. When no merger takes place the vortices merely rotate around each other undergoing a kind of pulsation in their shape. The interesting dynamics takes place when the two vortices actually merge.

We present here a number of runs that were conducted at much higher resolution than the runs of the previous section (the need for doing this will become apparent in a moment). They all have the identical initial condition $d_{c}=2.2$, and differ in their values of $\gamma$ and $\delta$.

The first one, which establishes a reference point, is essentially an Euler merger since it occurs at $\delta=0$ and $\gamma=10^{-3}$ (see Figure 2.13). The distinguishing feature of the Euler merger is the shedding of very thin and elongated vorticity filaments as the vorticity field proceeds to settle into an axisymmetric state (cf. the third frame of the first column in Figure 1.4, which, it is valuable to recall, is a spectral simulation). As was shown by Melander et al. (1988) the shedding of these vorticity filaments is an integral part of the axisymmetrization process.

Consider next the run shown in Figure 2.14, for which $\delta=0$ and $\gamma=1$. The main qualitative behavior is not dissimilar, with the exception that the vorticity filaments seem to exhibit a tendency to "roll up" into pools of vorticity. The final configuration resembles more to a three-vortex state, as opposed to a single vortex surrounded by filaments. We note that a qualitatively similar result has recently been obtained by Williams and Wilson (1988) in their finite-difference simulation of Jovian vortices (cf. in particular their Figure 7). Although their system is not quasigeostrophic (they solved the full shallow water equations with one layer), their parameter ranges are such that geostrophy is the prevailing balance in the flow. 
Keeping the value of $\delta$ fixed, we show in Figure 2.15, an equivalent barotropic merger at $\gamma=3$. It is truly remarkable how drastically the behavior changes at this higher value of $\gamma$. Note that here the Greens' function is identically equal to $-K_{0}(\gamma r)$ since the logarithmic component is completely absent from the equivalent barotropic case. The generation of filaments is suppressed and axisymmetrization does not take placet.

A similar result is shown for the even larger value of $\gamma=5$ in Figure 2.16. It is interesting to see how local the interaction is in these cases. Consider for instance the $T=24$ frame of Figure 2.16. It is obvious that only the side of each vortex that is closest to the other vortex is affected, while the rest of the vortex is essentially not moving. Moreover, the rotation rate of the two vortices about their common centroid is much slower than the merging process (presumably by a factor $\gamma^{-3}$ ). The final vorticity distribution can then be described as an essentially elliptical vortex over which large amplitude nonlinear disturbances propagate without breaking.

Finally, consider a merger at the same "large" value of $\gamma$ but in the presence of a finite (though deep) lower layer, such as the one shown in Figure 2.17 for $\delta=0.2$. Although reduced by the small value of $\delta$, the presence of the logarithmic term in the Greens' function is clearly felt at long distances over which the $\mathrm{K}_{0}$ component has no effect. The shedding of vorticity takes place and, similarly to the results of Figure 2.14, leads to the formation of two smaller vortices that are simply advected by the flow of one large vortex.

The important conclusion to be retained is that, in the equivalent barotropic model at large $\gamma$, the filamentation process is greatly suppressed and, therefore, axisymmetrization cannot take place. As we will show in detail in Chapter 4 , this can be described by the fact that the stagnation points in the

\footnotetext{
T Only a single surgery has been "manually" performed in this and the next run, to remove a common boundary between the two vortices. This step is necessary in order to carry the integration out to the times of $O\left(\gamma^{3}\right)$. Dritschel (priv. comm.) has repeated some of these runs at even higher resolution and shown that extremely thin and convoluted filaments are generated in the very high curvature region where one vortex "wraps around" the other. However these are so small that several magnifications are necessary to see them. Moreover their presence increases so much the computational burden that he was unable to carry to integration out beyond the very early times. The surgery we have performed eliminates these events on very small scales and allows us to compute to times over which the large scale features evolve significantly. The conclusions we draw in this section are therefore not valid for the extremely small scales. Whether such very small scale processes are physically relevant, in view of the inevitable presence of dissipation in any realistic system, is not entirely clear. it would be of great interest to repeat some of these calculations with a continuous model, e.g. by doing high resolution spectral simulations, to establish whether the nonaxisymmetrizing behavior persists in the case where the vorticity fields are smooth.
} 
corotating streamfunction never succeed in penetrating the vorticity boundary and extracting filaments.

\subsection{Doubly-Connected Rotating One-Layer V-states}

\subsubsection{A Review of the Saffman-Szeto Approach}

Before proceeding to the study of situations in which the geostrophic potential vorticity is present in both layers, we conclude this chapter by explicitly showing, qualitatively and quantitatively, how the shapes of the curves in Figures $2.10,11$ and 12 for the critical merger distance as a function of $\gamma$ and $\delta$ can be understood in connection with the existence of corotating V-states. What we have done is to generalize to the two-layer system, the study that Saffman and Szeto (1980) conducted for Euler equations.

As we have already mentioned in the review of Section 1.2, $\mathrm{N}$ vortices of equal and constant vorticity can be arranged in a symmetric configuration in such a way that they are stationary in some rotating frame. The simplest case is the one where two vortices simply rotate around each other without change in shape; this combination has been referred to as the doubly-connected rotating $V$-state. From intuitive geometrical considerations, it shouldn't be surprising that the existence and properties of these $V$-states are intimately connected with the occurrence of merger.

The basic idea is the following: the initial condition that is usually chosen to study the merger problem, namely two exact circles of equal vorticity, can be considered to represent a large amplitude perturbation of a doubly-connected $\mathrm{V}$ state, if one exists for that value of $d_{c}$, the distance between the centroids scaled by the radius of each vortex. This ratio (centroid distance over radius) is the relevant parameter which characterizes the initial condition of the merger problem. Of course the doubly-connected $V$-states are not circular, but the quantity $d_{c}$ can be calculated by defining the equivalent radius as the square root of the area over $\pi$. If a V-state does not exist at that value of $d_{c}$, or if it is unstable, it is reasonable to expect the initial condition to evolve towards merger of the two vortices. Otherwise the system will evolve by "oscillating" (both in physical and in phase space) around this stable stationary state. 
Let us consider first the question of existence. When the vortices are far apart, it is certainly possible to find a $\mathrm{V}$-state and one can easily guess that the shapes will be not be very different from circulart; its value of $d_{c}$ is large since the centroid separation is much bigger than the radius. As the vortices are brought closer together they become more elliptical, in order to resist each other's shear, and the value of $d_{c}$ decreases. It is obvious that the vortices can be brought no closer then when they are actually touching at one point. That last - "limiting" - Vstate has a specific, presumably $O(1)$, value of $d_{c}$, let's call it $d_{L}$, and no $V$-state exists for $d_{c}<d_{L}$.

This is the essence of the idea of Saffman and Szeto. Merger will occur for any value of $d_{C}<d_{L}$ because no $V$-state that exists to play the role of a stationary point to oscillate around in phase space. It turns out that, for the Euler equations, one doesn't even need to calculate the exact $V$-states. By approximating them with ellipses (which is equivalent to doing a second order moment model) and solving a single very simple nonlinear algebraic equation Saffman and Szeto found $d_{L} \approx 3.0$. They also numerically solved the full integro-differential system to determine the shapes of the $V$-states and found $d_{L} \approx 3.16$.

Before showing our own results for the two-layer model, we need to say a word about stability. Since even when a V-state exists it need not be stable, $d_{L}$ will in fact represent only a lower bound to the critical merger distance. However it so happens that, with the exception of the very elongated and "cuspy" states close to the limiting one, most doubly-connected rotating $V$-states are probably stable, so that $d_{L}$ turns out to be a very good lower bound for the critical merger distance.

More specifically, consider the schematic drawing of a doubly-connected state shown in Figure 2.18. Of the inner and outer radii, $x_{A}$ and $x_{B}$ respectively, we can, without loss of generality, chose $x_{B}=1$ and define the ratio $x_{A} / x_{B} \equiv v$ as single the parameter of interest. By a very careful numerical study of the eigenvalue problem for the linear stability, Dritschel (1985) showed that, for the Euler equations, only the $m=2$ mode ( $m$ being the discrete angular wave number of a perturbation on the contour shape) can be linearly unstable, and that this occurs when $v<0.083$, i.e. when the ratio of the inner to the outer radius is less than 1:10.

This is, of course, only a linear result, but it certainly provides a first step towards an understanding of why the value of $d_{L}$ is so close to the critical merger distance. Alternatively, and perhaps more interestingly, it might not be erroneous to

T This was proven analytically by Dritschel (1985) for the Euler equations. 
claim that the proximity of $d_{L}$ to the critical merger distance is, rather, an indication of the nonlinear stability of most doubly-connected rotating V-states. What we are going to show next is that these same considerations can be applied, with only quantitative differences, to the merger problem for the quasigeostrophic two-layer model.

\subsubsection{Two-Layer Model Results}

Since we are only interested in the properties of the upper-layer doublyconnected rotating $\mathrm{V}$-states in as much as they lead us, by considering the limiting cases (i.e. the ones for which the two vortices are touching at the origin) to a better understanding of the variation of the critical merger distance as a function of the two parameters of the two-layer model $-\gamma$ and $\delta$, we have only briefly considered the cases for which $v \neq 0$.

In general, both qualitatively and quantitatively, the resultst are perfectly analogous to the ones obtained for the other types of upper-layer V-states presented in section 2.2 namely that, for fixed $v$ and $\delta$, the difference from the Euler shapes is very small and is maximal around $\gamma=O(1)$ for $\delta \neq 0$, with the equivalent barotropic model not reducing to the Euler limit at $\gamma>1$.

As an example we show, in Figure 2.19 a set of $V$-states at $\gamma=1$ and $\delta=1$ (equal layer depths) as we decrease the value of the ratio $v$ of the inner to the outer radius from 1 to 0 . Some of their properties are given in Table 2.1, including the angular velocity $(\Omega)$, the area, the equivalent radius $(R)$, the centroid of each vortex $(\bar{x})$ and the ratio of the centroid distance to the equivalent radius $\left(d_{c}\right)$. $A$ second example, for the equivalent barotropic model at large $\gamma$, is shown in Figure 2.20 , where the $V$-states for $\gamma=10$ and $\delta=0$ are drawn for the same values of $v$ (see Table 2.2 for their properties).

Since our aim is to compare the the values of $d_{c}$ with the results of the merger problem for which the circles in the initial condition have area equal to $\pi$ (while the area of the $V$-states is in general different from $\pi$ ), a rescaling is necessary to make the comparison meaningful. The way to proceed is the following: for any given $\gamma, \delta$ and $v$, we find a $V$-state of area $A$ for which the equivalent radius is $R$ and the intercentroid distance $d_{c}$. If we want rescale lengths

t Details of the algorithm, and, particularly, the method with which we have dealt with the infinite curvatures of the limiting states that are of primary interest here, are contained in Appendix B3. 
by an amount $\alpha$ so that the area of each vortex is equal to $\pi$, we must chose $\alpha=(A / \pi)^{1 / 2}$. The value of $\gamma$ too then needs to be rescaled so that the $V$-state of area $\pi$ will occur at $\gamma^{*}=\alpha \gamma$. The value of $\gamma^{*}$ is also given in Tables 2.1 and 2.2 for each $V$ state.

Let's now finally concentrate exclusively on the limiting $V$-states. i.e. the ones for which $v=0+$.For the three "canonical" values of $\delta(0,0.2$ and 1$)$ we have plotted the values of $d_{c}$ versus $\left(\gamma^{*}\right)^{-1}$ in Figure 2.21. It is remarkable how closely these curves compare with ones for the critical merger distance obtained in Section 2.3.2. In particular, for the case $\delta=1$ the value of $d_{c}$ is contained between 3.11 and 3.19 over the whole $\gamma$ range, and that is the reason why we were unable to detect a dependence of the critical merger curve on $\gamma$ at $\delta=1$ with a grid spacing of 0.2 in $d_{c}$ (cf. Figure 2.10).

For $\delta=0.2$ we find $3.0<\mathrm{d}_{\mathrm{c}}<3.2$, which agrees well with the variations of the critical merger distance with $\gamma$ shown in Figure 2.11. Moreover the location of the minimum in $d_{c}$, which occurs around $\gamma \approx 0.6$, can be reconciled with the coarse results of the initial value calculations. Notice that the values of $d_{c}$ are always below 3.3, indicating the probable instability of the cuspy $V$-states near $v=0$. Finally, for the equivalent barotropic case, comparison on Figures 2.12 and 2.21 shows good agreement, though we have been unable to extend the curve to very high $\gamma$ because of convergence problems in that limit, as we have already mentioned.

In conclusion, we would like to restate in a different, and perhaps more illuminating, way what we believe to be the fundamental reason for the existence of a critical distance for the merger problem: when two regions of equal vorticity are sufficiently separated they fail to merge because of the presence "in their vicinity" (in some phase space with some measure) of a stationary and stable configuration that acts as an attractor for the vorticity field, preventing the collapse of the two vortices into a single one. The collapse inevitably occurs where the initial condition is "far" from any stable stationary state. We think it is reasonable to conjecture that this understanding of the fundamental underlying dynamics of the merger problem can be generalized to make it applicable to continuous distributions fo vorticity as well as to continously stratified quasigeostrophic flows.

\footnotetext{
$\dagger$ Numerically, of course, we cannot set $v \equiv 0$. We have determined the solution at $v=0.001$, which, as can be seen from Tables 2.1 and 2.2 is more than adequate for our present purposes.
} 


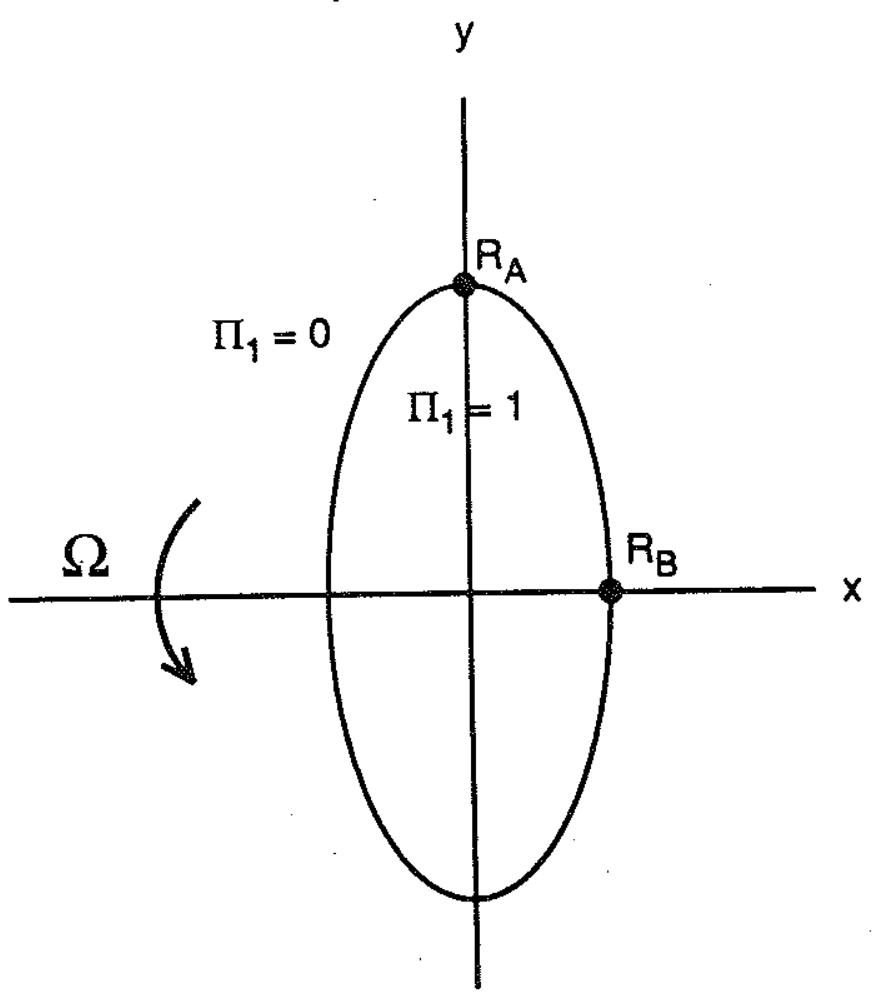

FIGURE 2.1. Schematic drawing of an upper-layer rotating V-state. Only the upper layer is shown here (recall that $\Pi_{2}=0$ ). 

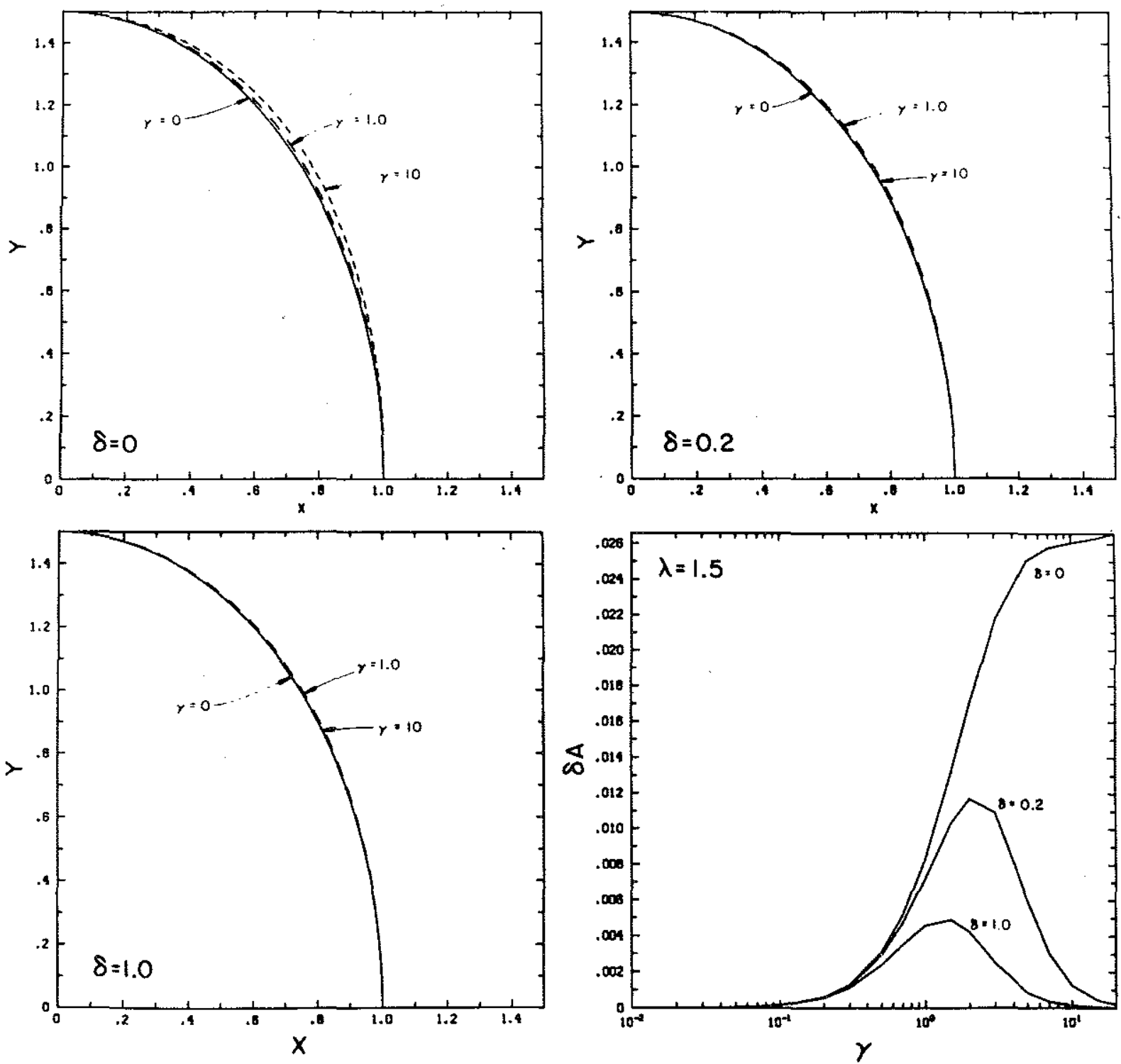

FIGURE 2.2. The upper-layer rotating $V$-states for $\lambda=1.5$, and $\delta=0,0.2$ and 1 . The values of $\gamma$ are indicated for each vortex. The lower right figure is a plot of the relative change in area versus $\gamma$, as explained in the text. 

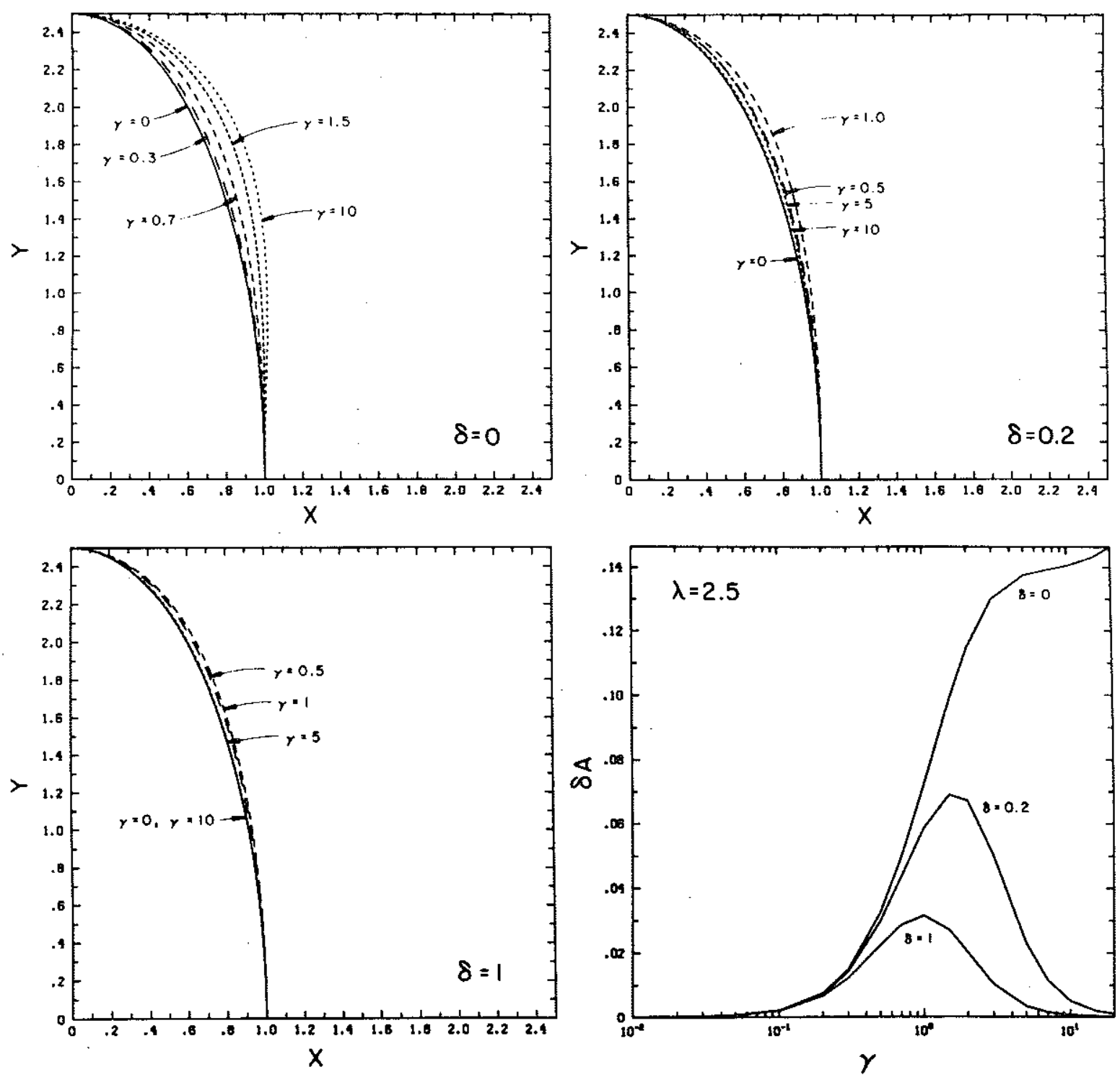

FIGURE 2.3. The upper-layer rotating $V$-states for $\lambda=2.5$, and $\delta=0,0.2$ and 1 . The values of $\gamma$ are indicated for each vortex. The lower right figure is a plot of the relative change in area versus $\gamma$, as explained in the text. 

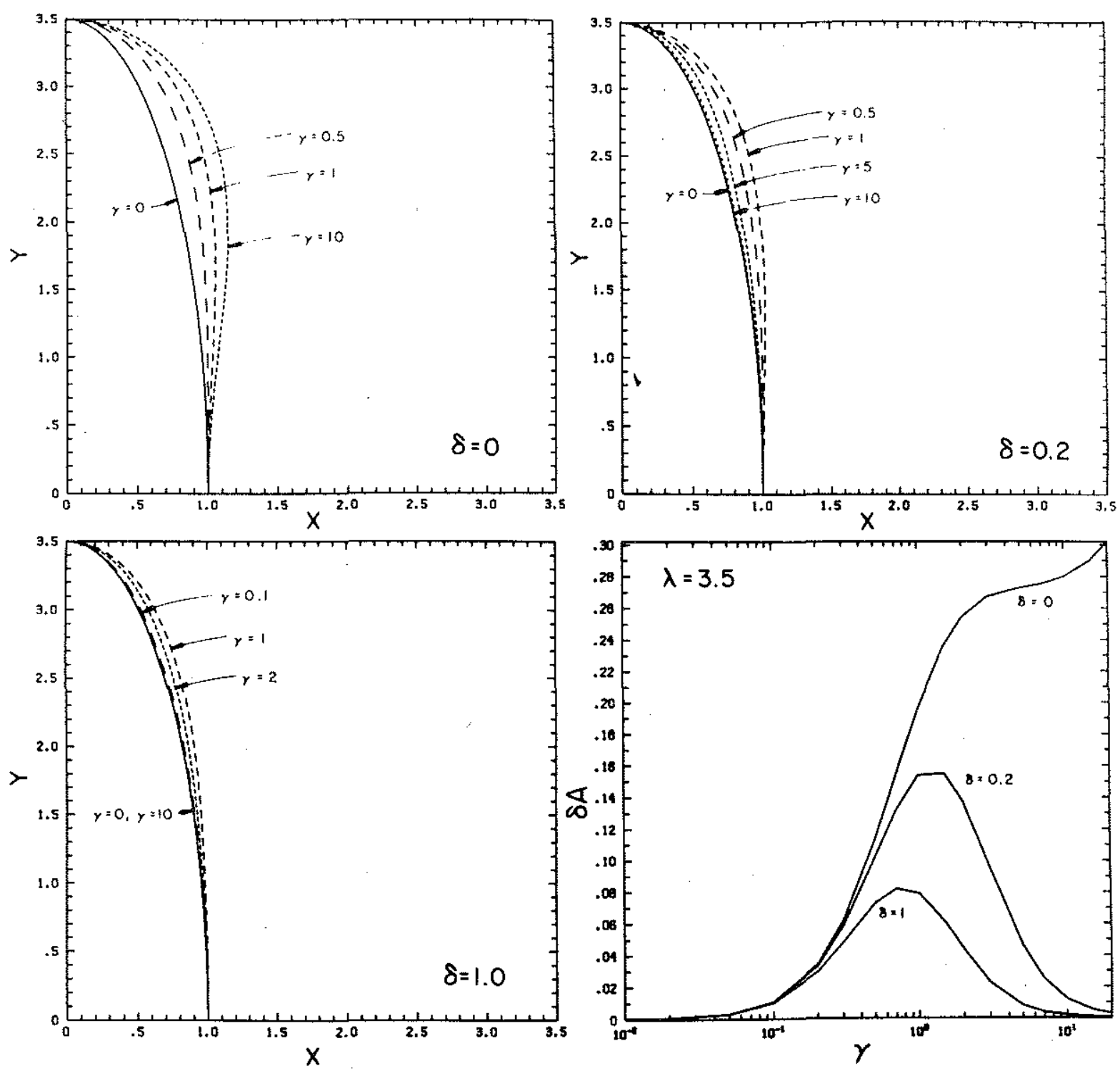

FIGURE 2.4. The upper-layer rotating $V$-states for $\lambda=3.5$, and $\delta=0,0.2$ and 1. The values of $\gamma$ are indicated for each vortex. The lower right figure is a plot of the relative change in area versus $\gamma$, as explained in the text. 

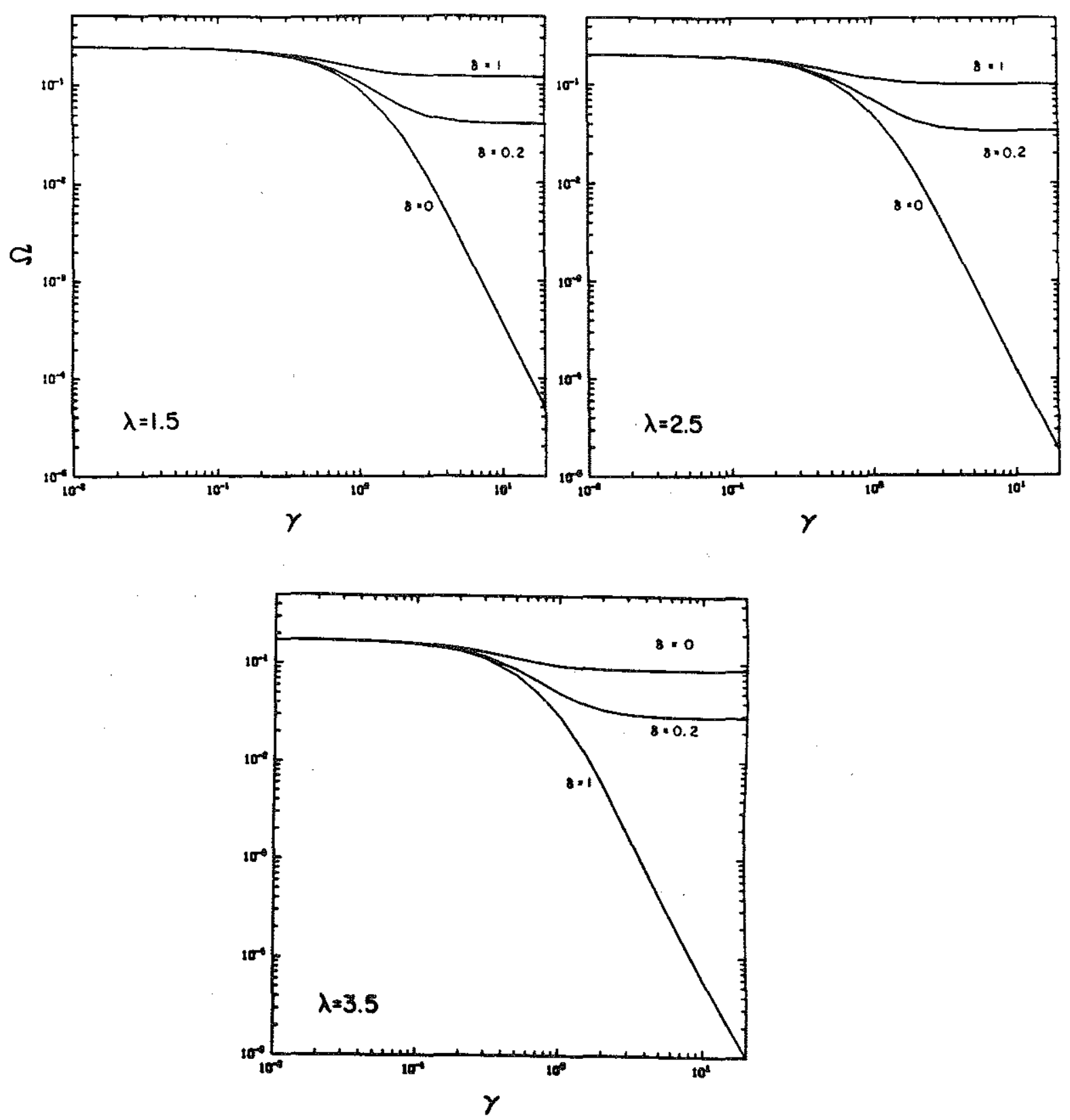

FIGURE 2.5. The angular velocity $\Omega$ of the upper-layer rotating $V$-states vs. $\gamma$ for $\delta=0,0.2$ and 1 , and for $\lambda=1.5,2.5$ and 3.5 for the $V$-states shown in Figs. 2.2-2.4 


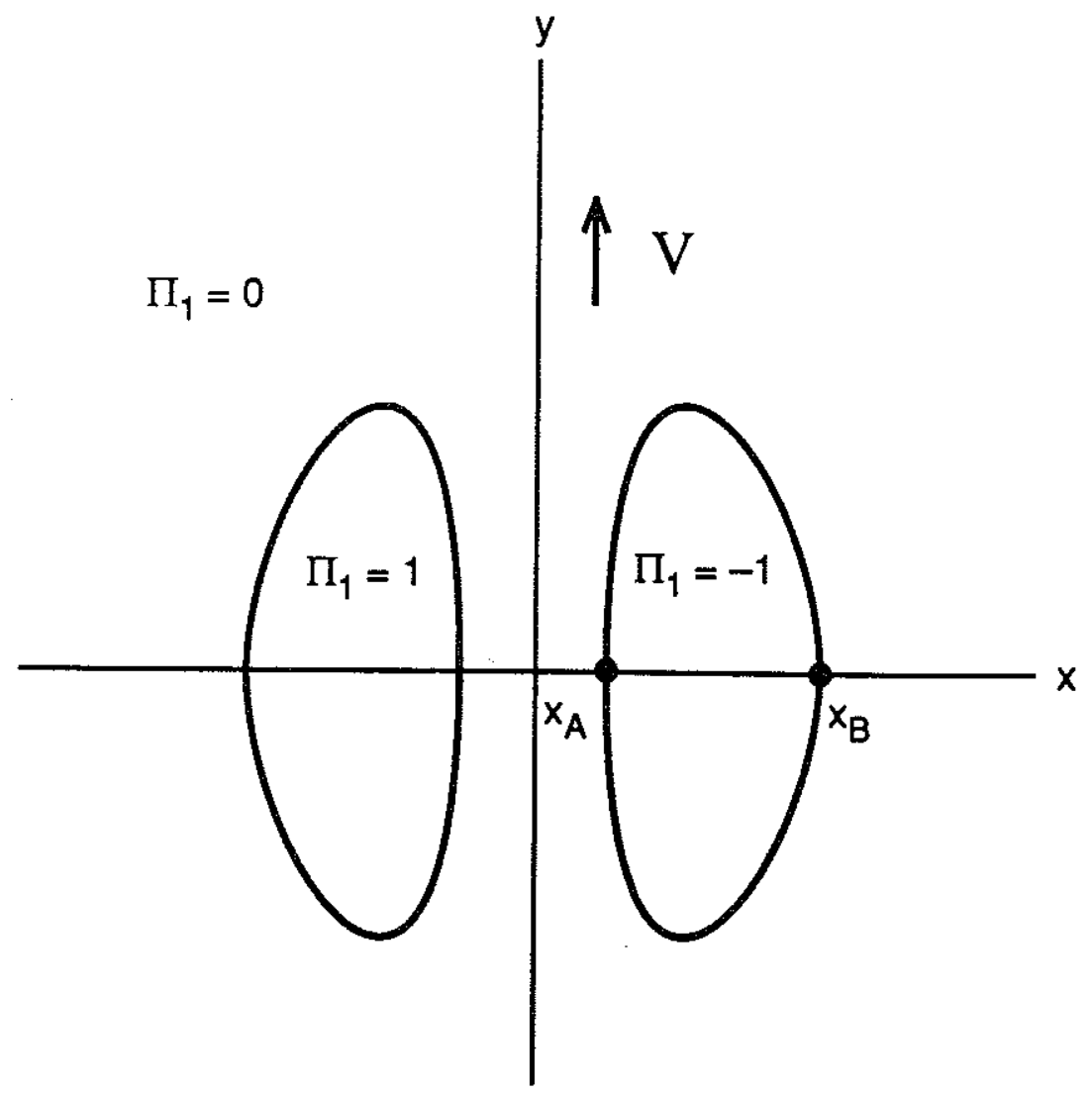

FIGURE 2.6. Schematic drawing of an upper-layer translating dipolar V-state. 

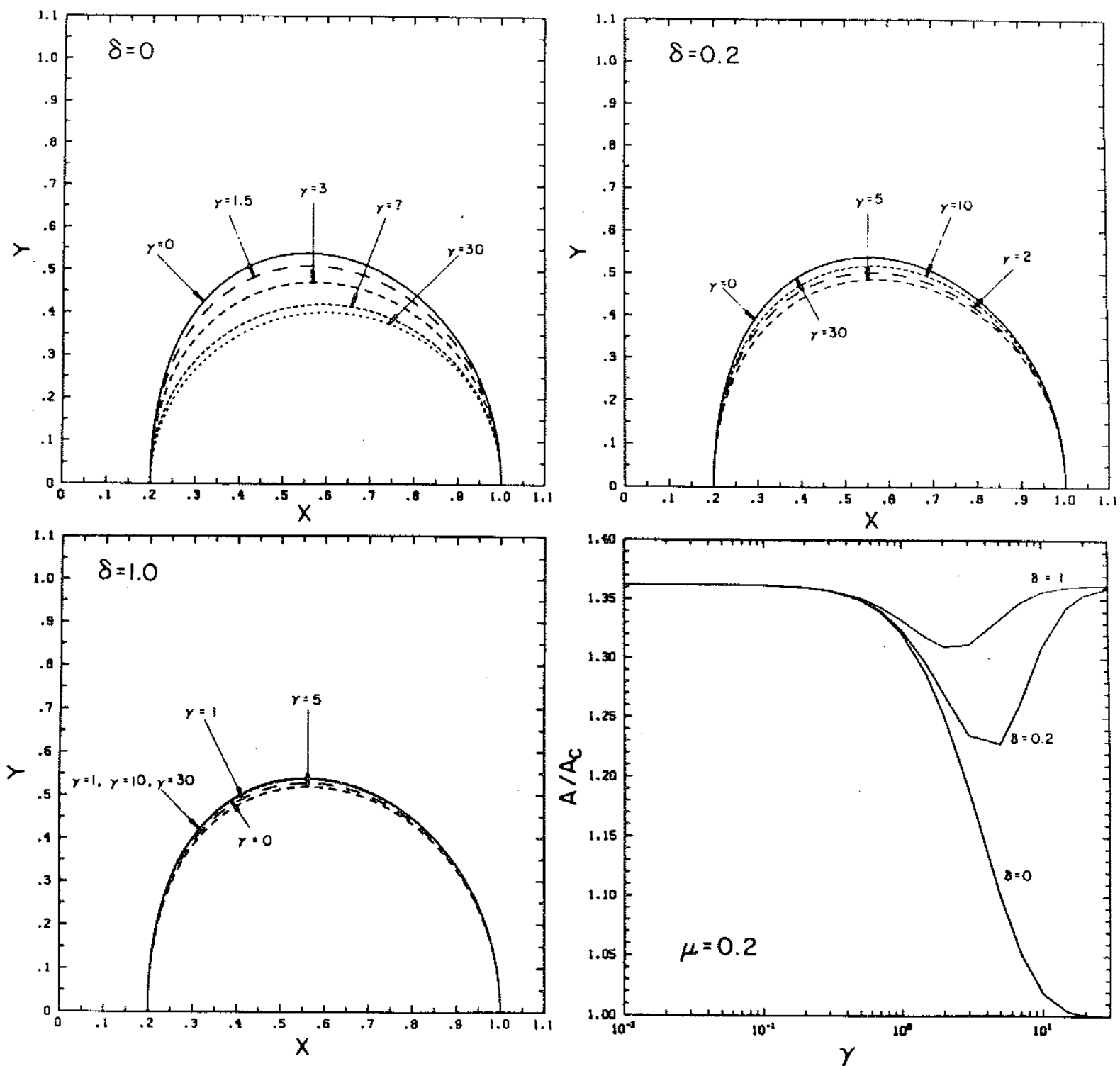

FIGURE 2.7. The upper-layer dipolar $V$-states for $\mu=0.2$, and $\delta=0,0.2$ and 1 . The values of $\gamma$ are indicated for each vortex. The lower right figure is a plot of the relative change in area versus $\gamma$, as explained in the text. 

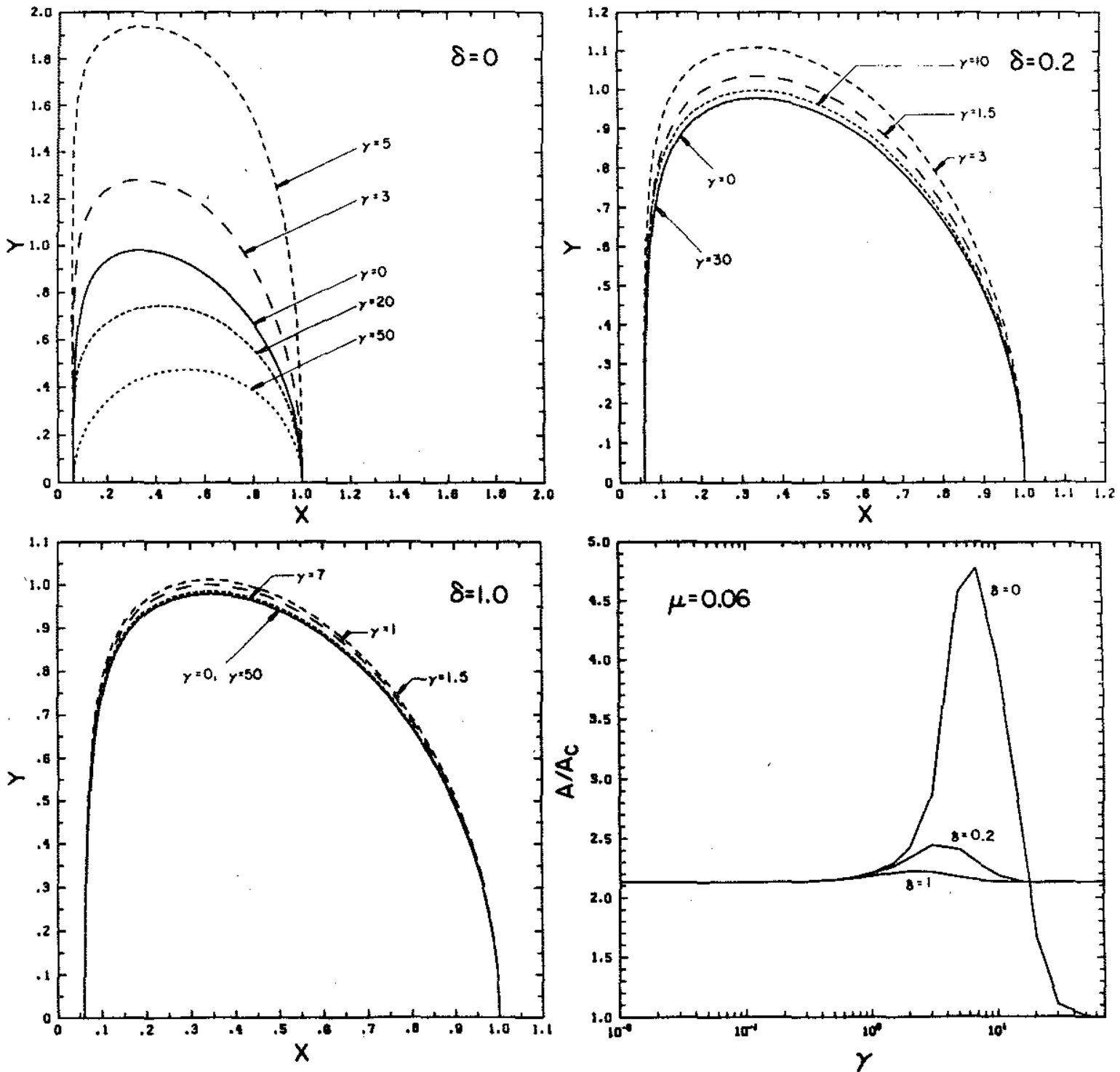

FIGURE 2.8. The upper-layer dipolar $V$-states for $\mu=0.06$, and $\delta=0,0.2$ and 1. The values of $\gamma$ are indicated for each vortex. The lower right figure is a plot of the relative change in area versus $\gamma$, as explained in the text. 

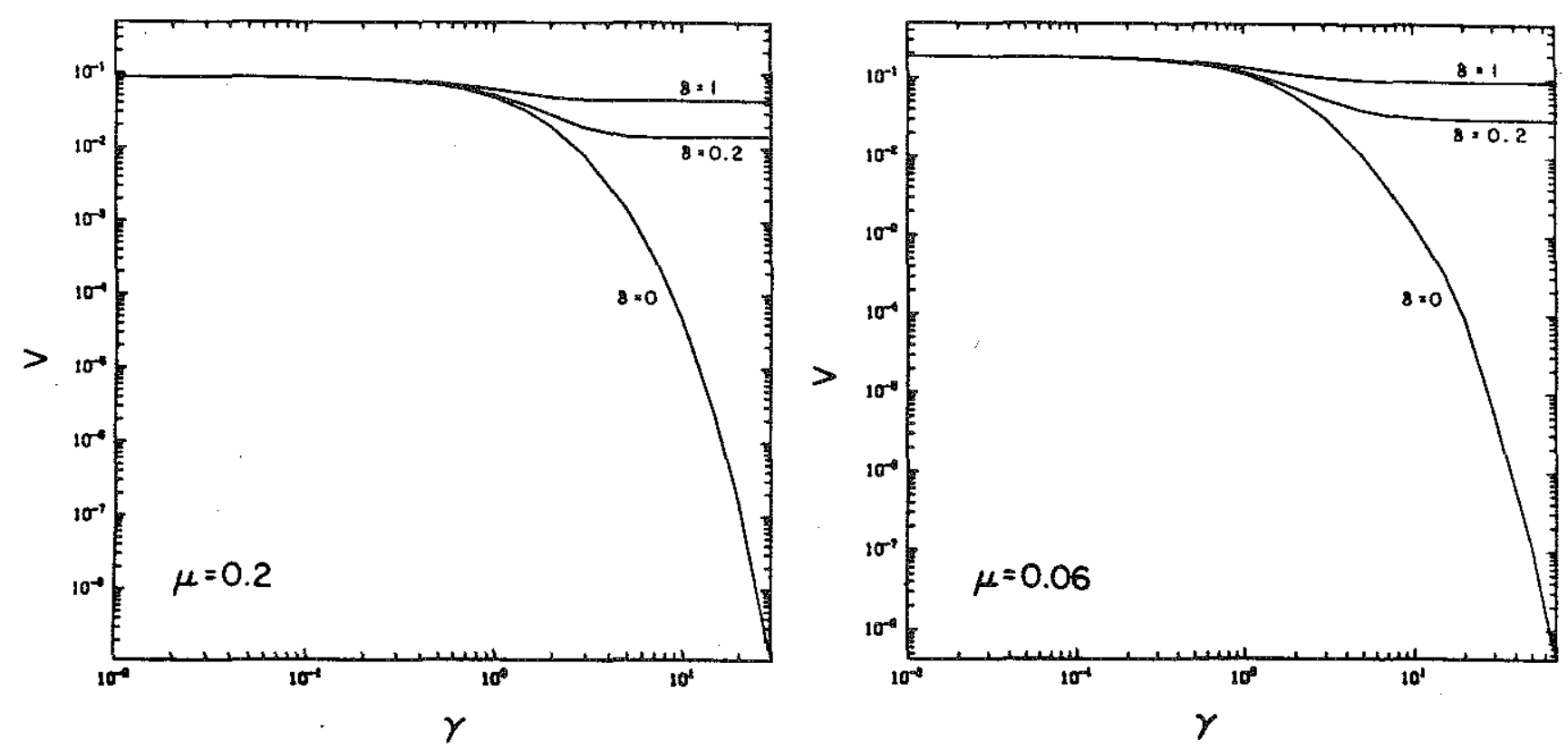

FIGURE 2.9. The linear velocity $V$ of translation of the upper-layer dipolar $V$-states vs. $\gamma$ for $\delta=0,0.2$ and 1 , and for $\mu=0.2$ and 0.06 for the $V$-states shown in Figs. 2.7 and 2.8 . 


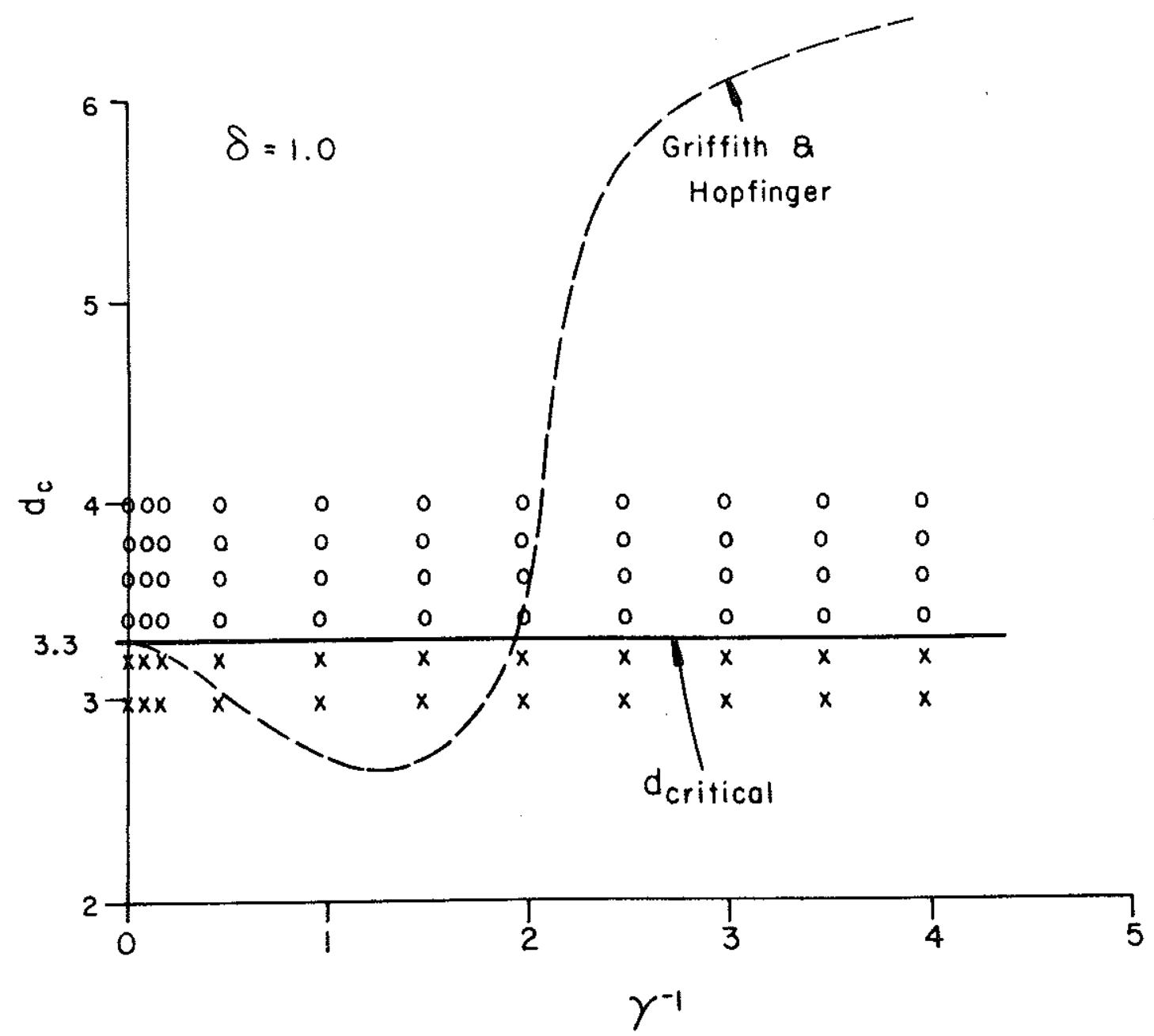

FIGURE 2.10. Merger results for the case of two equal layers, $\delta=1$, in the $\left(d_{c}, \gamma\right)$ parameter space. $A \times$ indicates that merger occurred at the correspoding values of $d_{c}$ and $\gamma$, while $a$ o indicates that no merger was observed. The solid line for $d_{c r i t i c a l}$ was drawn in by hand to help visualize the regions of merger and no-merger. 


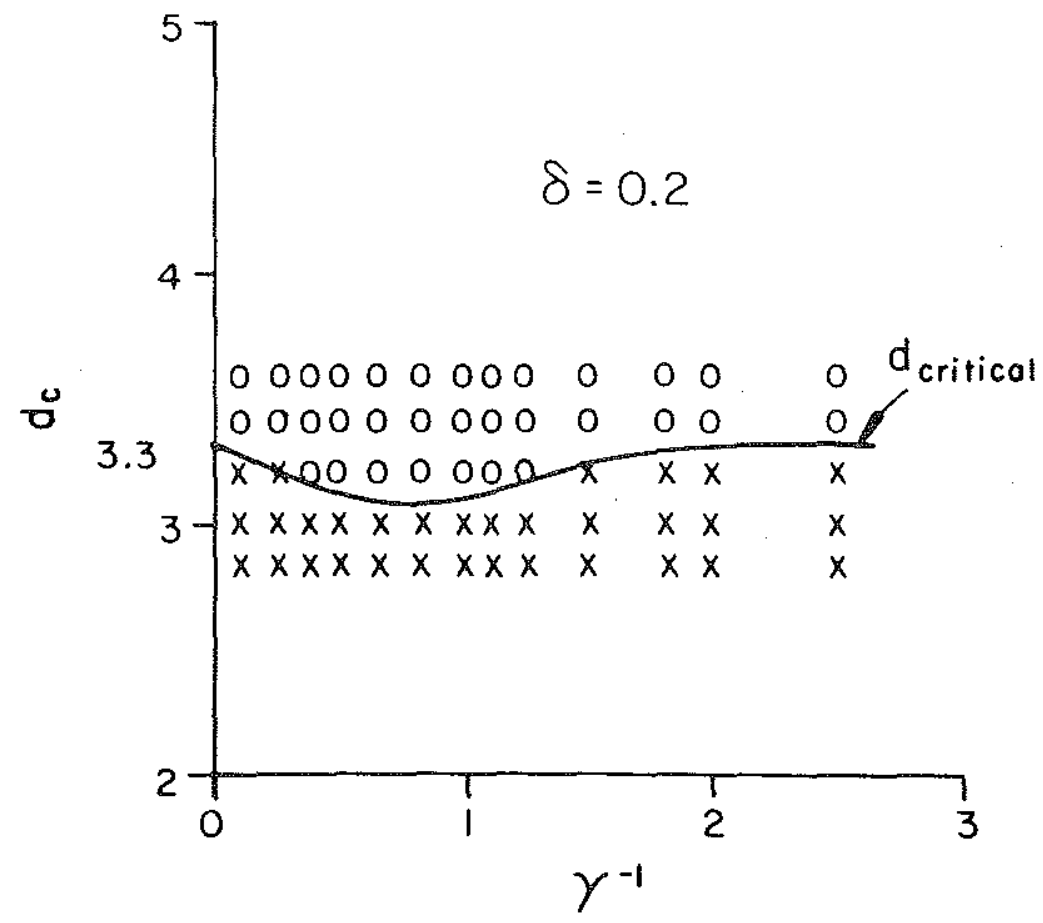

FIGURE 2.11. Same as Figure 2.10 but for $\delta=0.2$.

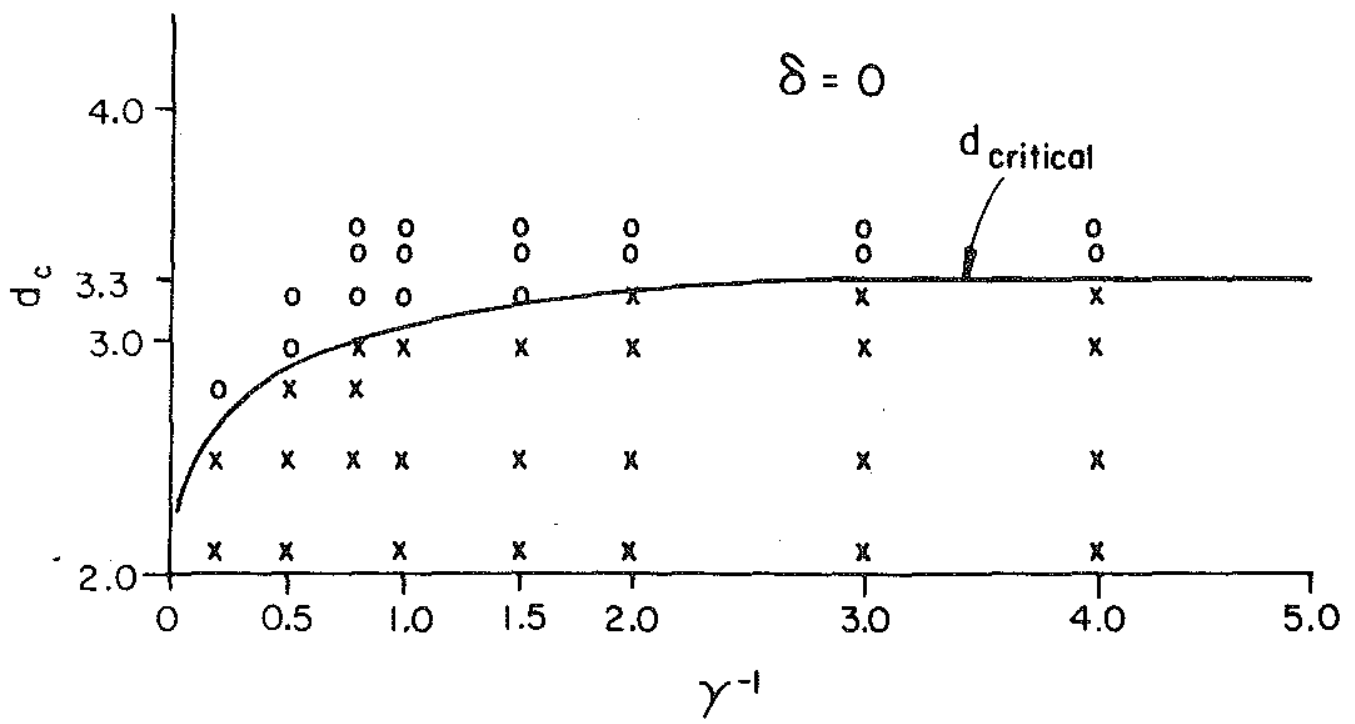

FIGURE 2.12. Same as Figure 2.10 but for $\delta=0$. 
$T=0$
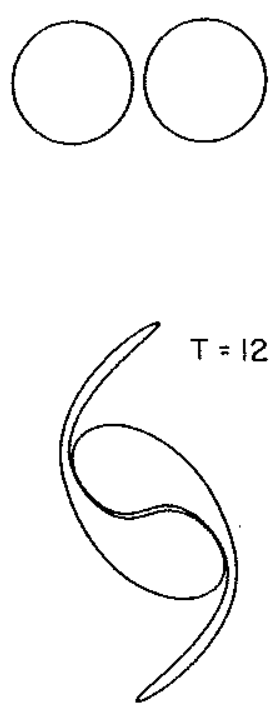

$T=3$
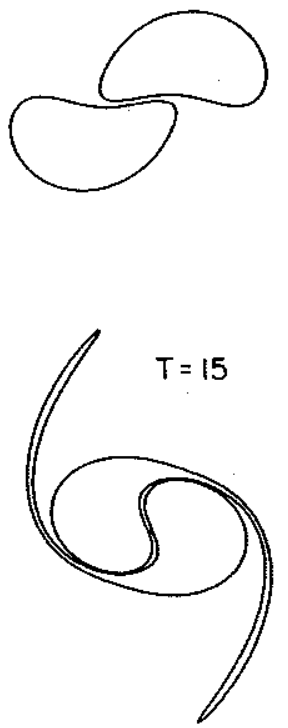
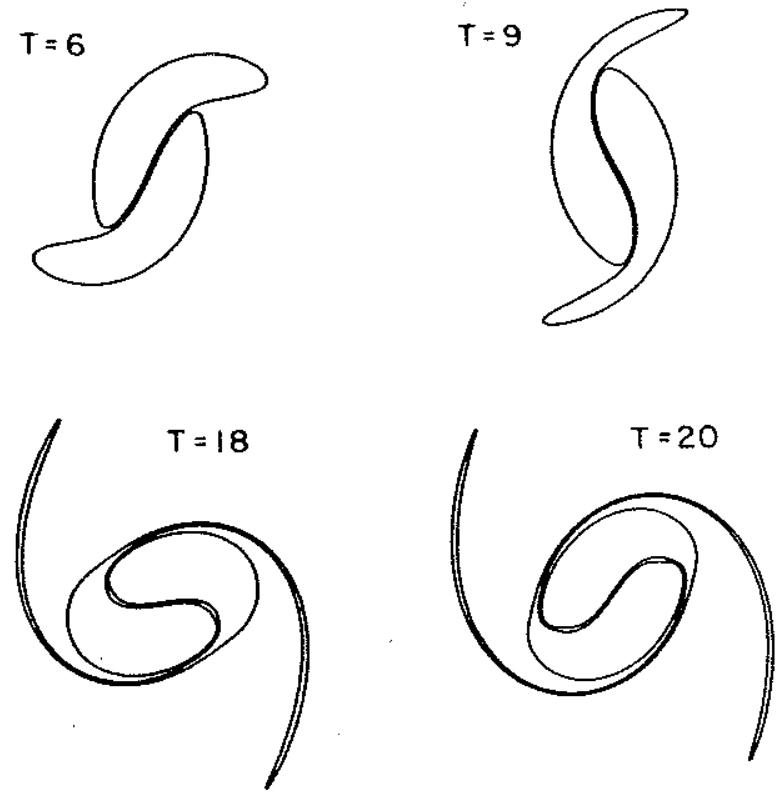

FIGURE 2.13. A merger at $\delta=0$ and $\gamma=10^{-3}$ for $d_{c}=2$.2. Notice the formation of thin filaments and the axisymmetrization of the core, typical of the Euler equations. 

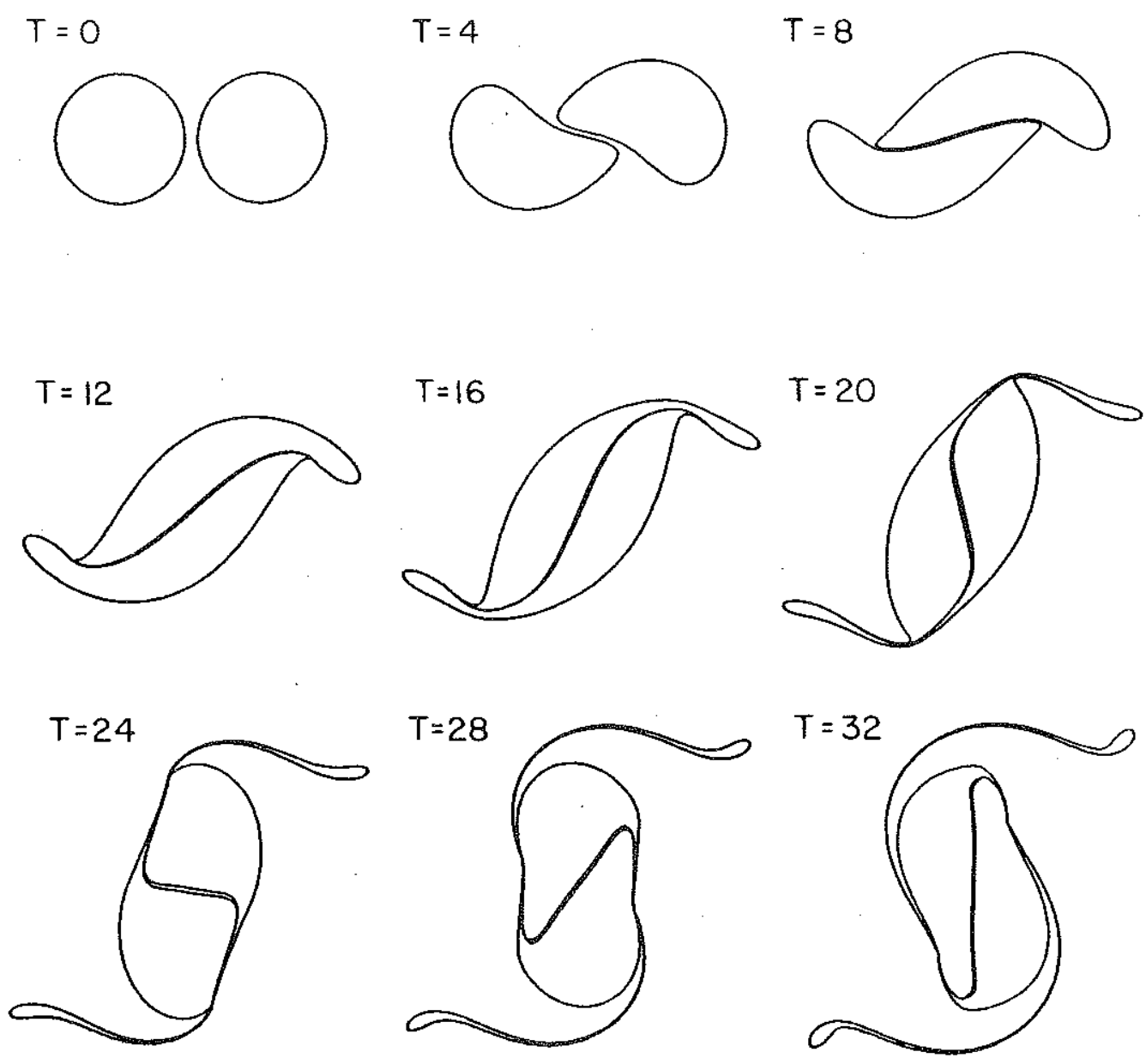

FIGURE 2.14. A merger at $\delta=0$ and $\gamma=1$ for $d_{c}=2.2$. Notice the condensation ("rollup") of vorticity in the filaments, similar the one observed by Williams and Wilson (1988). 

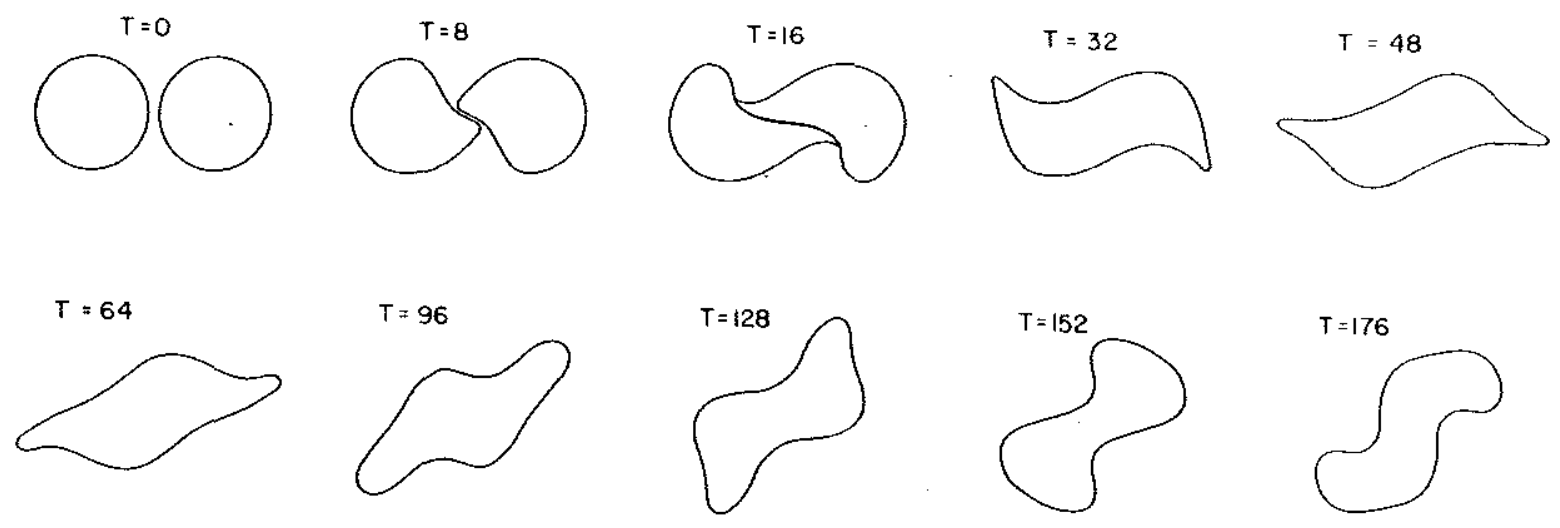

FIGURE 2.15. An equivalent barotropic "large $\gamma$ " merger $\left[\delta=0, \gamma=3\right.$ and $\left.d_{c}=2.2\right]$. Notice the absence of filamentation and the non-axisymmetrization of the vorticity.
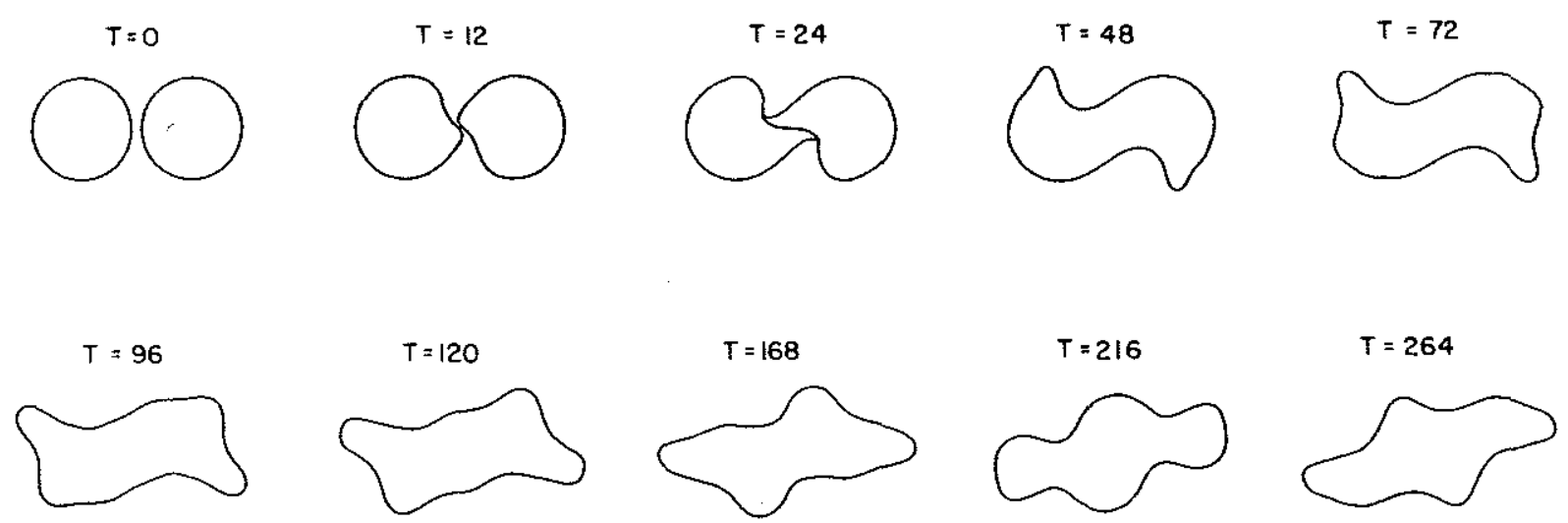

FIGURE 2.16. Same as Figure 2.15 but for $\gamma=5$. 

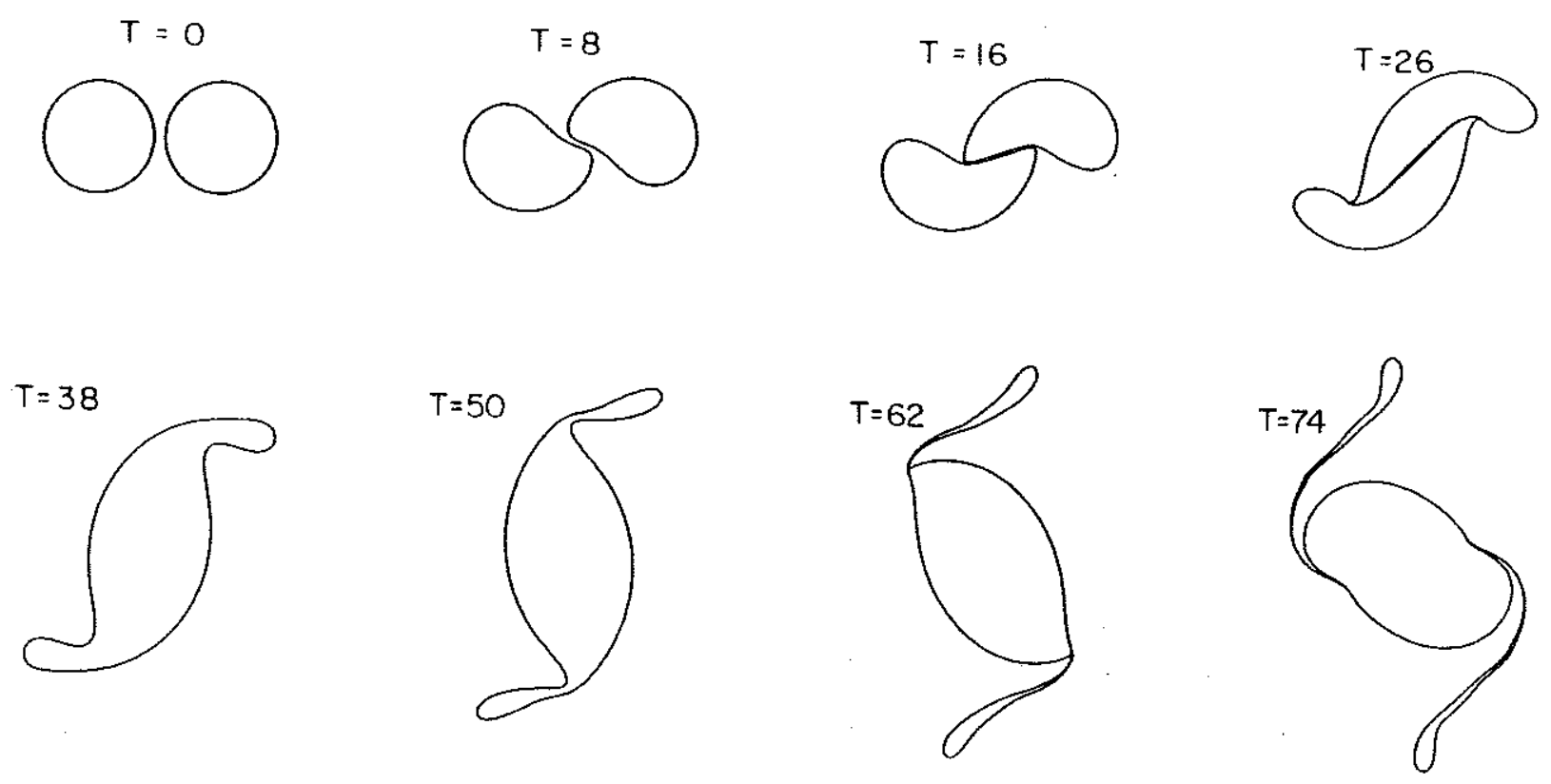

FIGURE 2.17. A merger at large $\gamma$ but finite $\delta$ [ here $\delta=0.2, \gamma=5$ and $d_{C}=2.2$ ]. 


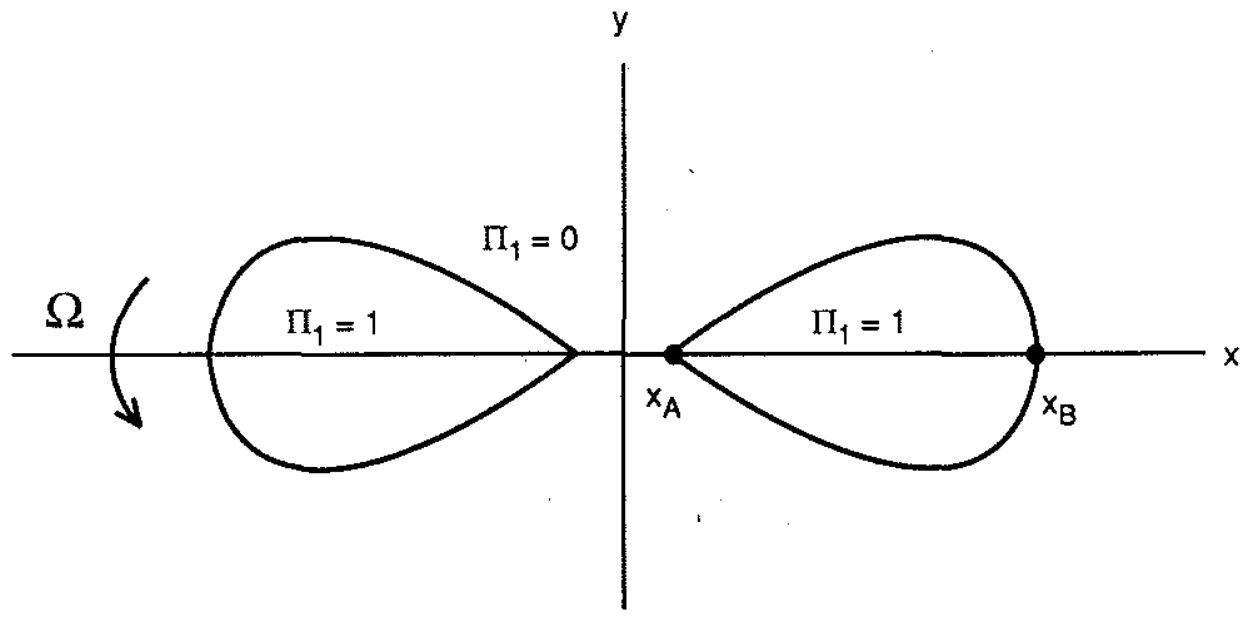

FIGURE 2.18. Schematic drawing of a doubly-connected rotating $V$-state in the upper layer. Recall that $\Pi_{2}=0$ here. 


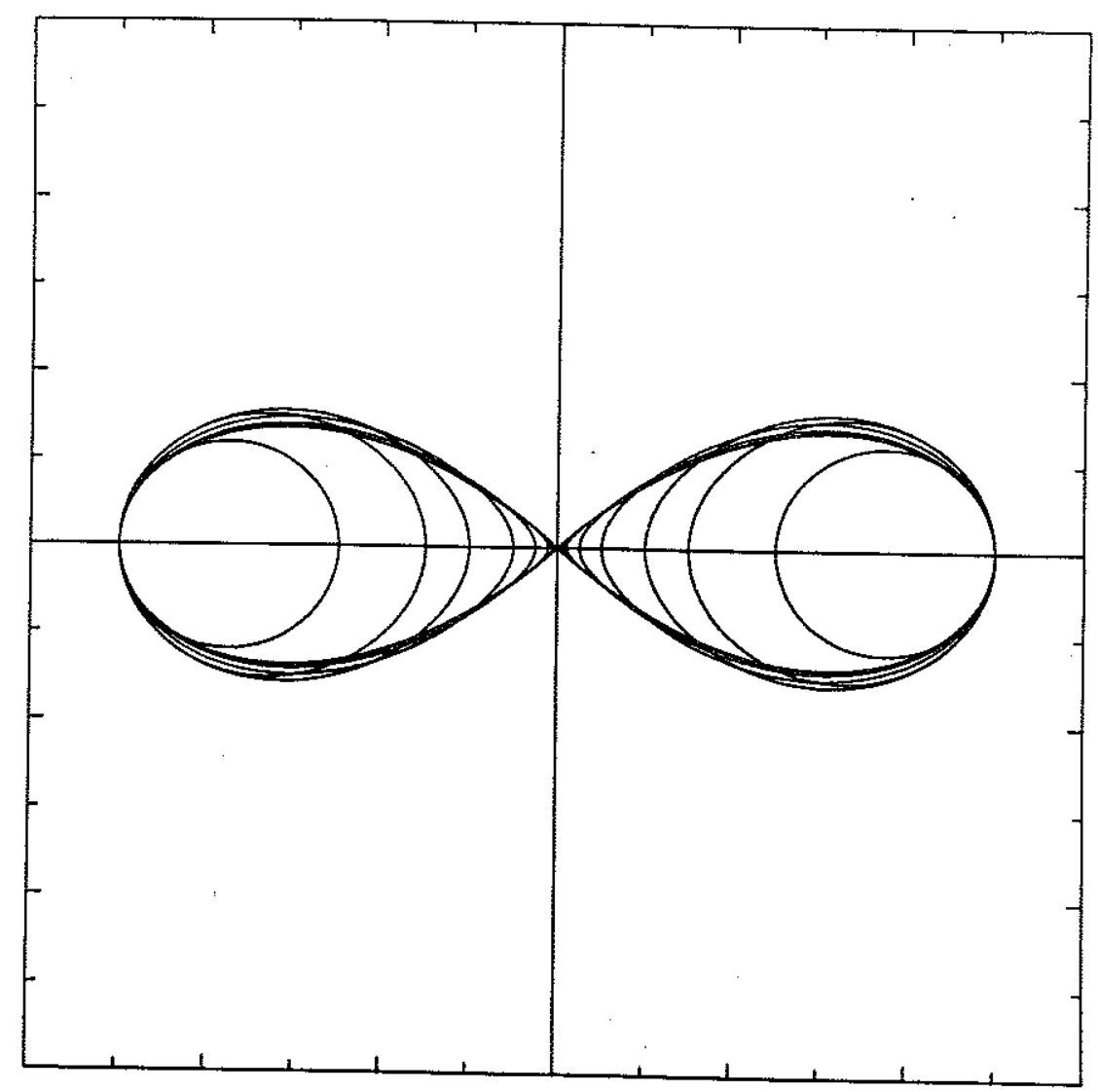

FIGURE 2.19. Doubly-connected rotating upper-layer $V$-states for $\delta=1$ and $\gamma=1$.

TABLE 2.1

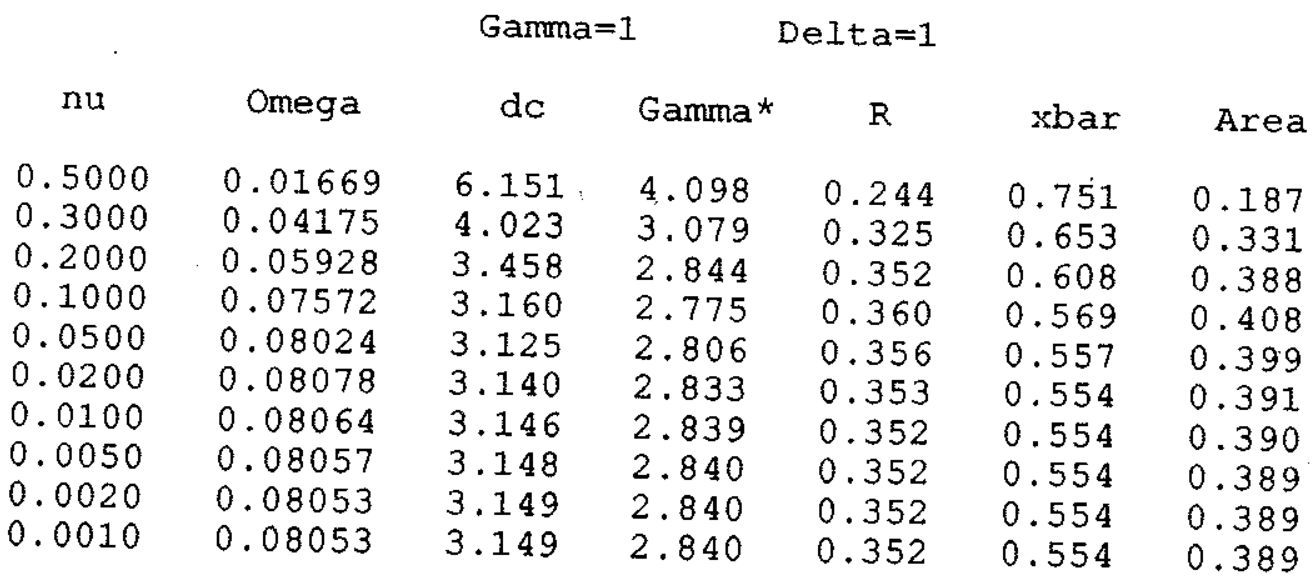




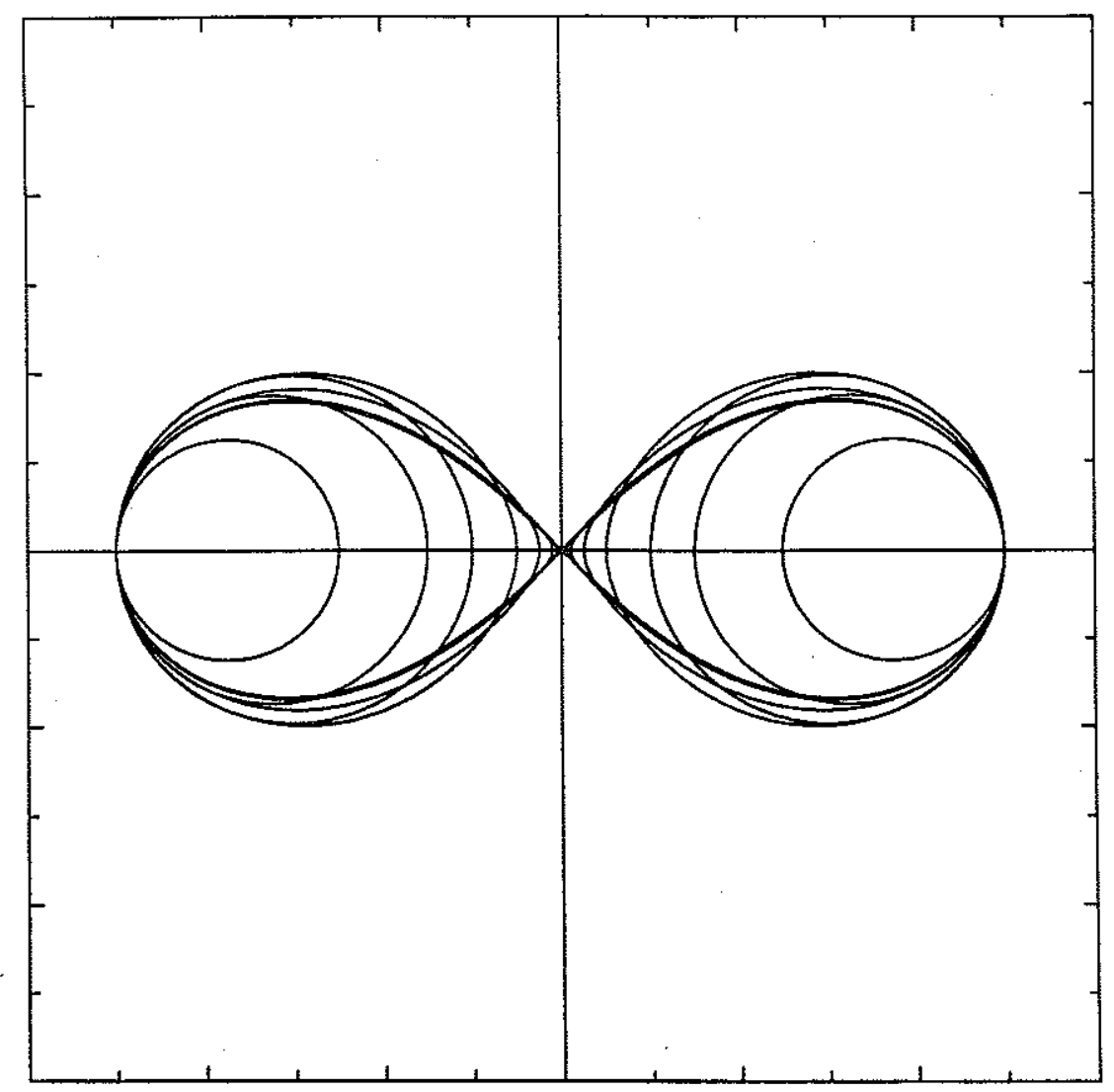

FIGURE 2.20. Doubly-connected rotating upper-layer $V$-states for $\delta=0$ and $\gamma=10$.

TABLE 2.2

\begin{tabular}{|c|c|c|c|c|c|c|}
\hline \multirow[b]{2}{*}{ nu } & \multicolumn{3}{|c|}{ Gamma $=10$} & \multicolumn{2}{|c|}{$\operatorname{Delt} \mathrm{a}=0$} & \multirow[b]{2}{*}{ Area } \\
\hline & Omega & $d c$ & Gamma * & $\mathrm{R}$ & xbar & \\
\hline 0.5000 & 0.00000 & 6.005 & 0.400 & 0.250 & 0.750 & 0.196 \\
\hline 0.3000 & 0.00001 & 3.721 & 0.286 & 0.349 & 0.650 & 0.383 \\
\hline .2000 & 0.00007 & 3.034 & 0.252 & 0.396 & 0.601 & 0.493 \\
\hline 0.1000 & 0.00046 & 2.666 & 0.238 & 0.420 & 0.560 & 0.555 \\
\hline 0.0500 & 0.00092 & 2.713 & 0.246 & 0.406 & 0.551 & 0.519 \\
\hline 0.0200 & 0.00117 & 2.819 & 0.255 & 0.392 & 0.553 & 0.484 \\
\hline 0.0100 & 0.00120 & 2.848 & 0.257 & 0.389 & 0.555 & 0.477 \\
\hline 0.0050 & 0.00121 & 2.856 & 0.257 & 0.389 & 0.555 & 0.475 \\
\hline 0.0020 & 0.00120 & 2.857 & 0.257 & 0.389 & 0.555 & 0.475 \\
\hline 0.0010 & 0.00120 & 2.857 & 0.257 & 0.389 & 0.555 & 0.475 \\
\hline
\end{tabular}



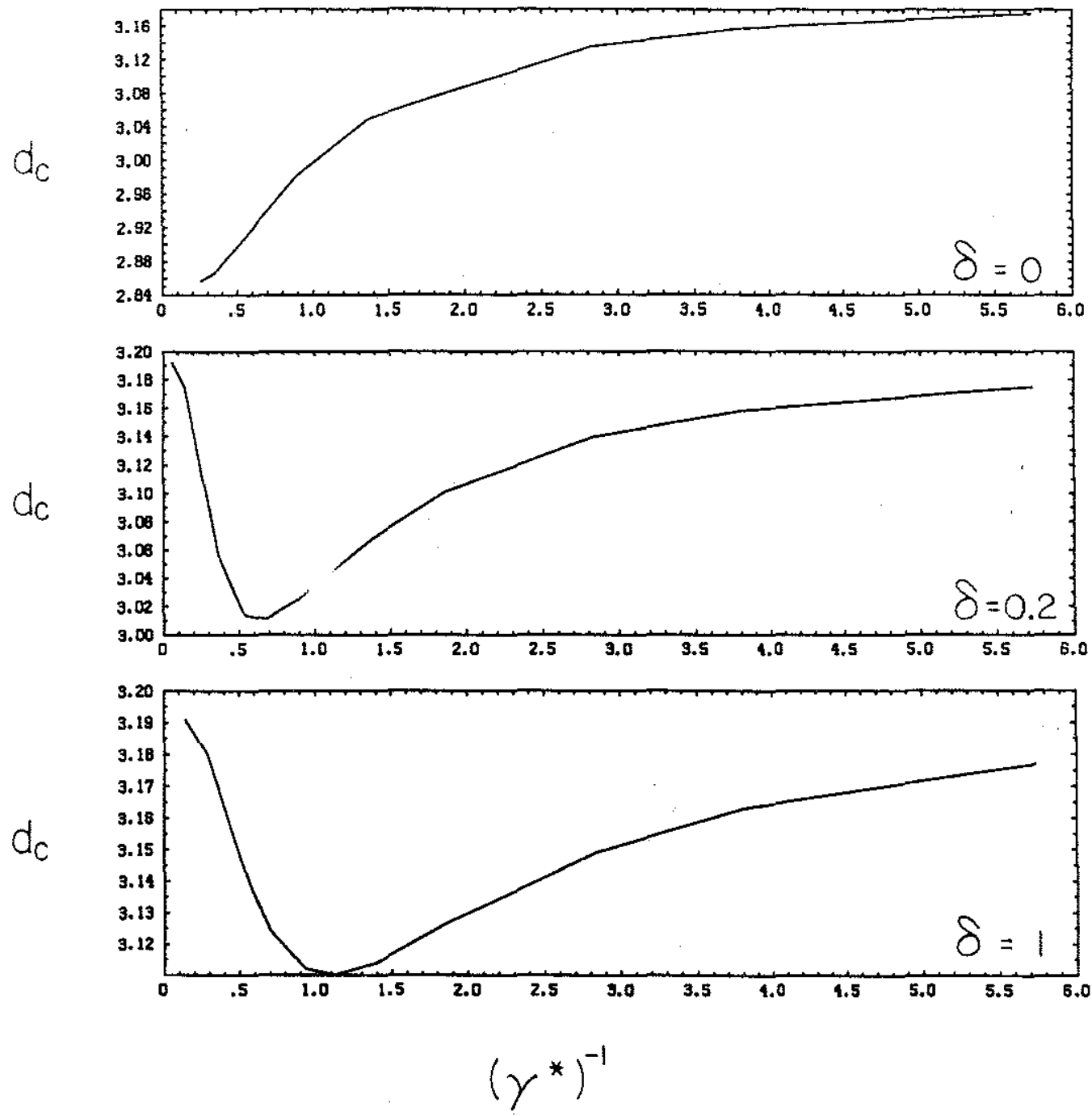

FIGURE 2.21. Ratio of the intercentroid distance to the equivalent radius for the limiting doubly-connected rotating upper-layer $V$-states as a function of $\gamma^{*}$ for $\delta=0,0.2$ and 1 . 


\section{Two-Layer Dynamics with Geostrophic Potential Vorticity in Both Layers}

Roll on, thou deep and dark blue ocean - roll!

Byron

We now lift the constraint $\Pi_{2}=0$, and turn our attention to problems where the geostrophic potential vorticity is simultaneously present in both layers. In this case the number of interesting configurations that deserve to be studied becomes very large. Rather than to concern ourselves superficially with a large number of situations, we have opted to investigate in depth a small number of fundamental problems. Moreover, we eliminate one dimension of the parameter space at the outset by limiting our investigations throughout this chapter to the case of two layers of equal depth $(\delta=1)$.

It should therefore be apparent that, even in the present simplest context of finite-area f-plane inviscid two-layer dynamics, many important questions still need to be addressed, and that the problems that we have tackled and solved in this thesis represent, in some sense, only a first taste of the very rich and fascinating dynamics of strongly interacting highly-nonlinear potential vorticity structures in a stratified quasi-geostrophic fluid.

Two recent studies have attracted our attention in particular, and have played a dominant role in directing our efforts in two specific directions. The first is a set of very high resolution numerical simulations of turbulence decay in a stratified quasigeostrophic system (McWilliams 1988) which have pointed to the question of alignment as potentially one of the most important type of vortex interaction within a quasigeostrophic stratified fluid. We have thus been led to study the alignment problem in the simple two-layer quasigeostrophic model, and the results of that investigation are presented in the next section (Section 3.1).

We have already alluded to the fact that, in analogy to the one-layer merger, the phenomenology of the alignment problem is strongly connected with existence of stationary configurations of vorticity. This connection is made explicit in Section 
3.2, where the new and rich phenomenology introduced by the presence of vorticity at different densities is presented. Above all, we will show that much insight can be gained from a new geometrical approach to the understanding of the dynamics of vorticity structures.

The last portion of this chapter (Section 3.3) was motivated by a number of recent studies dealing with the formation and the evolution of translating pairs (dipoles) of vorticity in a quasigeostrophic two-layer model. We have determined the stationary shapes of finite-area constant-vorticity dipoles with one vortex in each layer. Again we are going to show that a geometrical interpretation of the results can be quite valuable in understanding of the presence of boundaries that delimit the existence of these solutions to certain regions of parameter space.

\subsection{The Alignment Problem in the 2-Layer Model}

High-resolution numerical simulations of two-dimensional turbulence (Fornberg 1977, Basdevant et al. 1981, McWilliams 1984, Babiano et al. 1986) have shown how the enstrophy spectra in the inertial range appear to be considerably steeper than the the $\mathrm{k}^{-1}$ slope predicted by the self-similar Kolmogorov theory of Kraichnan (1967). The discrepancy is due to the failure of that theory to take into account the existence of the energy invariant and the localness of the flow dynamics in physical space.

These are known to lead to the condensation of vorticity into strong coherent isolated and long-lived vortex structures, superimposed on a weak vorticity continuum that behaves like a passive scalar (see Babiano et al. 1986, for more details). The fundamental mechanism through which this coalescence of vorticity takes place is the merger of vorticity regions of the same sign. It is through merger that the reverse energy cascade to the small wavenumbers is understood to occur.

The status of our knowledge of stratified geostrophic turbulence is not as advanced, mostly due to a lack of sufficiently high-resolution numerical simulations that are just now beginning to appear. The original observation of Charney (1971) that the geostrophic expression of the potential vorticity is isomorphic to the twodimensional (relative) vorticity $\nabla^{2} \psi$ thereby implying a $k^{-3}$ spectral lawt, reverse

t Here $k$ is the total three dimensional wavenumber, with the vertical coordinate rescaled by the ratio $N / f$ of the Brunt-Väissälä frequency to the Coriolis parameter. 
energy and forward enstrophy cascades and equipartition between potential and kinetic energy has been generally confirmed by the study of Herring (1980), who used a statistical closure model and, more recently, by the intermediate resolution numerical simulations of Hua and Haidvogel (1986).

A number of other investigations have instead concentrated on models with low vertical resolution, and in particular the two-layer model (Rhines 1979, Salmon 1980 and Holloway 1986). The important results to emerge from those studies are that interactions of barotropic and baroclinic modes lead to energy cascades in opposite directions - a barotropic "red" cascade to the large scales (analogous to the two-dimensional case) and a baroclinic cascade to the smaller scales (a manifestation of baroclinic instability). Moreover intermodal interactions tend to transfer energy from the baroclinic to the barotropic modes, and occur preferentially at scales close to the radius of deformation.

The first suggestion of the emergence of isolated and coherent vortices in stratified quasigeostrophic turbulence was reported by Hua and Haidvogel (1986) and a clear indication of their presence is found in the very recent numerical simulation of McWilliams (1988) at an unprecedently high resolution. In particular, McWilliams reports that the vortices of stratified geostrophic turbulence seem to be mostly elongated in the vertical direction and horizontally axisymmetric.

We identify the mechanism responsible for the the formation of stratified geostrophic vortices with the alignment process defined in Chapter 2. In the same way as merger is responsible for the reverse energy cascade of the barotropic modes towards the larger scales, alignment is the fundamenatal mechanism that mediates the conversion of energy from baroclinic to barotropic modes.

The alignment process necessitates the existence of stratification, and the simplest possible context in which it can be studied is the one we have chosen for this thesis: the quasigeostrophic two-layer model. Since this is new phenomenon (we are unaware of previous direct studies of vortex alignment) we will start first by defining it in an unambiguous way, and then proceed to a qualitative exploration of its phenomenology.

We consider the interaction of two circular regions of equal vorticity located one in the upper and one in the lower layer, and whose centroids are separated at $t=0$ by a horizontal distance $d_{c}$ (scaled by the radius of the vortices). If, for $t>0$, the centroid separation collapses to a lower value within a time of the order of the period $t_{c}$ of rotation of the vortices about their common centroid, we say that the two 
vortices have "aligned". When the vortices do not align, the centroid separation undergoes a periodic (or quasiperiodic) oscillation but, on average, does not decrease with timet.

The parameter space for the alignment problem, as we have defined it, is two-dimensional (recall that we are considering here only the simplest case $\delta=1$ of equal depth layers), since both the initial intercentroid distance $d_{c}$ and $\gamma$, the ratio of the radius of the vortices to the radius of deformation, can be specified independently. We start by presenting a number of different cases that give a first taste of the rich phenomenology of the alignment problem.

A first example of alignment is shown in Figure 3.1 for the case $d_{c}=2.2$ and $\gamma=1.25$, where the dashed (solid) line represents the lower (upper) -layer vortex. Notice how different the evolution of the vorticity field is from a merger event, in a much as the two vortices do not wrap around one another but simply come to a quasi-equilibrium state (in terms of not shedding further large vorticity filaments) as they rest on top of each other. The baroclinic configuration at $t=0$ has thus transformed itself into a essentially barotropic one. In Figure 3.1a we plot the intercentroid distance as a function of time; it can be seen to fall essentially from the initial value of 2.2 to about $0.6 \ddagger$.

As the coupling between the two-layers is reduced, it is reasonable to expect that, at some sufficiently small value of $\gamma$, alignment will cease to occur. In Figure 3.2 we illustrate this with a run at the same initial distance $d_{c}=2.2$ but at the smaller value of $\gamma=1.0$; notice how the intercentroid distance (Figure 3.2a) undergoes a quasiperiodic oscillation, and the originally non-overlapping vortices pulsate as if oscillating around a stationary vorticity configuration (a V-state). We will show in the next section that such $V$-states do indeed exist, and the result of Figure 3.2 seems to indicate that at least some of them are also stable.

Because the vortices are in different layers, they can actually be started off with a nonzero overlap. If the value of $\gamma$ is now held constant, one would probably expect that as the initial intercentroid distance is reduced alignment takes place. We show in 3.3 one such example at the same value of $\gamma=1$ and at the smaller value of

† The question of possible exact recurrence in the case of non-merger (or non-alignment) has not been investigated in detail even for the Euler equations. Suffice it to say that, in the present context, we are only concerned in the short times of the order $t_{c}$ and possibly secular behavior is not of interest here since, in actual simulations of geostrophic turbulence, the encounters usually last less than $O\left(t_{c}\right)$.

F The discontinuity in the value of the intercentroid distance at $t \approx 36$ is due to the fact that, after the filaments are disconnected by the automatic surgery, the intercentroid distance is calculated between the two central vortices alone. 
$d_{c}=1.4$ (the intercentroid distance as a function of time is shown in Figure 3.3a). After shedding a relatively thin filament, the vortices seem again to oscillate around a stationary state, as evidenced by the nearly periodic pulsation in the value of the intercentroid distance after $t \approx 40$.

In view of our experience with the merger problem, we would probably expect that if we start the vortices at an even shorter distance for the same value of $\gamma$ alignment should again occur. It is therefore surprising to discover that this need not be the case, as evidenced by run shown in Figure 3.4, for which the vortices do not align at the smaller initial intercentroid distance of $d_{c}=1.0$ for the same value of $\gamma=1$. Again we interpret this as being due to the existence "near" the initial condition of a V-state that is probably stable in some non-linear sense. It is interesting to observe how no alignment takes place in spite of the large amount of overlap between the two vortices.

The results of our exploration of the $\left(\mathrm{d}_{c}, \gamma\right)$ parameter space to delimit the region where alignment occurs is illustrated in Figure 3.5, where we have actually plotted the results as function of $\gamma^{-1}$ to make them comparable with the merger curves of Chapter 2. As in that case, we have drawn a cross where we have observed alignment to occur and a circle where no alignment takes place (we have, of course, been able to ascertain this only partially, in the sense that one cannot wait indefinitely to convince oneself that alignment does not occur). The solid curve that separates the crosses from the dots was drawn in by hand, and it only serves the purpose of approximately indicating the location of a transition in behavior.

Before showing how the shape of this curve is intimately connected with the existence of two-layer corotating V-states, a few comments should be made to propose the plausibility of some of its characteristics. In the first place, it must be noted that it is not surprising to have found that at $\gamma<\gamma_{\min }$ alignment never occurs; this simply reflects the fact that as the layers become sufficiently uncoupled the vortices cannot interact strongly enough to undergo an "irreversible" (more precisely "non-recurrent") evolution. Perhaps more surprising is that the value of $\gamma_{\text {min }}$ is extremely close to 1 .

The upper branch of the curve, say for $d_{c}>2$, can be understood easily in terms of arguments similar to the ones that were used to rationalize the merger curves. Thus as $\gamma \rightarrow \infty$ we expect the curve to tend towards the Euler $d_{c}$ value of 3.3, since in that limit the Green's functions of (1.4) become identically logarithmic (with the exception of an irrelevant time scale factor of $1 / 2$ for the case $\delta=1$ of interest 
here). In the large $\gamma$ limit then, when the vortices are not initially overlapping, the vorticity in each layer affects the flow as if it were present in both layers at the same time. A beautiful example of this interesting behavior is shown in Figure 3.6 where alignment is shown to occur at $\gamma=5$ and $d_{c}=2.2$. Note how small the overlap region between the two vortices is, in spite of the fact that they are actually located in different layers (the evolution could actually be mistaken for an Euler merger!). What is preventing the vortices from overlapping is the coupling between the layers that is so strong that it makes the flow in each layer behave as if both vortices were present in that layer.

The lower branch of the curve is also not entirely surprising. In a recent study on the linear stability of quasigeostrophic vortices in a two-layer model, Flierl (1988) has shown that barotropic circular vortices of constant potential vorticity are stable to non-axisymmetric baroclinic perturbations of mode $m=1$. At small $d_{c}$ it is not unreasonable to consider the initial condition we have used for the alignment problem as an $m=1$ baroclinic perturbation on a barotropic vortex, and thus we expect the alignment curve never to intersect the $d_{c}=0$ axis. What the results of figure 3.5 seem to indicate is that barotropic circular vortices are in fact nonlinearly unstable to quasigeostrophic baroclinic $m=1$ perturbations provided the amplitude is large enough and the radius of deformation is small.

We conclude this section with a last example of alignment that illustrates the variety of behavior that can occur when vorticity is present in both layers. In Figure 3.7 we show the evolution of two vortices with initial intercentroid distance $d_{c}=3.0$ at $\gamma=2$. The vortices are essentially split in half by their counterpart in the opposite layer and thereby lead to a final state composed of four vortices. This kind of complex final state can emerge because of the many degrees of freedom that are possible when vorticity is present in both layers, and it suggests that four-vortex stationary states exist and are probably stable. The two-vortex states are presented next. 


\subsection{Doubly-Connected Rotating Two-Layer V-States $†$}

The purpose of this section is to illustrate how the shape of the critical alignment distance curve (cf. Figure 3.5) can also be understood from a generalization of the arguments presented in Section 2.4. The basic idea is very simple: we wish to prove that alignment takes place in the region of the $\left(d_{c}, \gamma\right)$ plane where no stable doubly-connected rotating two-layer V-state exists.

Again we need to distinguish between the existence and the stability questions. We are going to show in what follows that doubly-connected rotating two-layer $\mathrm{V}$-states do not exist in a region coinciding to a large extent with the region where alignment occurs, and that the discrepancy between these two regions must be attributed to the probable instability of the $V$-states with large curvatures.

Because the critical alignment curve is not a single-valued function of $\gamma^{-1}$, the method of Section 2.4 has been modified as follows. Consider the schematic drawing of a two-layer doubly connected V-state is given in Figure 3.8. It consists of two regions of equal geostrophic potential vorticity located one in the upper and one in the lower layer, rotating around their common centroid (the origin) with an angular velocity $\Omega$. As for the one-layer doubly-connected states, the only geometrical parameter to be chosen is the ratio of inner to outer radius that we define as $v=x_{A} / x_{B}$; the novelty here is that the vortices, being in different layers, can overlap in which case $v$ becomes negative. Note that the interesting parameter range for $v$ is $-1<v<1$.

Since we have confined the problem to two equal layers $(\delta=1)$, we retain the two-fold symmetry of the vortices (about the $x$-axis) and the parameter space to be investigated is only two-dimensional. The question we wish to address here can then be reformulated as follows: for what values of $v$ and $\gamma$ does a V-state of the type shown in Figure 3.8 exist? Recall that when the vortices are in the same layer (cf. Section 2.4) solutions can be found for all $v>0$ and $\gamma$. In that case, as we have shown, the interesting states are the ones were the vortices are actually touching at one point (the "limiting" states).

T The details of the algorithm used to determine these V-states are found in Appendix B3. 
When the vortices are in different layers the meaning of "limiting" state needs to be understood in the original sense of Wu et al. (1984). Namely, suppose we are varying some parameter $\alpha$ (which identifies the state) and, having found a state at $\alpha=\alpha_{0}$, we can find states for increasing values of $\alpha$ up to $\alpha=\alpha_{\mathrm{e}}$. If no state exists for $\alpha=\alpha_{\theta}+\varepsilon$, no matter how small $\varepsilon$ is, we designate the state at $\alpha=\alpha_{\theta}$ by the term "limiting" state. For the Euler equations the parameter that indicates the existence of a limiting state is usually a geometrical one. Thus, for example, the limiting state for doubly-connected rotating Euler V-states occurs at $v=0$.

The reason for subjecting the reader to this somewhat formal definition resides in that we want to show next that, in the quasigeostrophic two-layer model, there exist limiting $V$-states in $\gamma$. More specifically, for a fixed ratio of inner to outer radius $v$, we will show that when the two-vortices ovelap $(v<0)$ the limiting state can occur at a finite value of $\gamma$. A limiting state exists even when $v>0$ (no overlap) but it is only approached asymptotically as $\gamma \rightarrow \infty$. The reason for proceeding by increasing $\gamma$ at fixed $v$ in the investigation of the $(v, \gamma)$ plane is obviously suggested by the single-valuedness of the critical alignment distance curve (cf. Figure 3.5) when $d_{c}$ is taken to be fixed instead of $\gamma$.

Ideally, one would like, with reference to Figure 3.5 , to fix $d_{c}$ and calculate $V$ states for increasing values of $\gamma$, showing that $V$-states cease to exist for $\gamma>\gamma_{e}$. Recall, however, that $d_{c}$ is, for a $V$-state, the ratio of the intercentroid distance to the equivalent radius, and the algorithm we have used to determine the $V$-states does not allow us to to prescribe these values a priori. We have therefore proceeded by finding states as $\gamma$ is increased from zero at fixed $v$, until, for $\gamma=\gamma_{c}$, our algorithm fails to converget. We then compute $d_{c}$ for the $V$-state at $\gamma=\gamma_{c}$.

These values are shown in Table 3.1, where the values of $\gamma_{c}$ have been rescaled by $(\text { Area } / \pi)^{1 / 2}$ (cf. Section 2.4 for details) to make the numbers comparable with the alignment problem of the previous section, where the vortices had been chosen to be circles of radius 1 . We have also plotted these values as crosses in the $\left(d_{c}, \gamma\right)$ plane, together with the critical alignment curve derived from the initial value problems presented earlier (see Figure 3.9).

The agreement is not as good as for the one-layer case, but undoubtedly the location where the V-states cease to exist is a good zeroth order predictor for

† Whether the value $\gamma_{c}$ at which the algorithm stops converging corresponds to the value $\gamma_{e}$ at which the Vstates stop existing is a very delicate question that we postpone to the latter part of this chapter. For most of the cases we have computational evidence that the two are identical, while for some other cases the question remains open. 
the region where alignment can be expected to occur. The hashed sector is the location where $\mathrm{V}$-states do exist but are probably unstable (in some nonlinear sense). As we are going to show next, this seems to be the case especially for those $\mathrm{V}$-states whose shapes include regions of very high curvature.

We cannot avoid commenting on the lower part of the diagram in Figure 3.9, where the results seem to indicate the existence of a small region in which V-states do not exist but alignment doesn't seem to take place. Among a number of explanations we suspect that the initial value problems were carried out at insufficiently high resolution to detect the formation of thin filaments. Another possibility may be offered by the existence of another kind of $\mathrm{V}$-states in that region, that we are unable to capture with the present algorithm.

It remains to be shown what causes the $V$-states to stop existing at a finite value of $\gamma$, and we proceed now to a careful examination of the $V$-states themselves in order to elucidate this matters. We have determined the solutions by increasing $\gamma$ from zero at fixed $v$. For $v>0$ the situation is quite simple because the vortices do not overlap. At $\gamma=0$ the shapes are exact circles and $\Omega=0$. As $\gamma$ is increased, i.e. as the coupling between the two layers is made stronger, the vortices become elongated, in a way similar to the Euler case, because, in order to remain stationary, they must resist the shear induced by the vortex in the opposite layer. When $\gamma$ becomes very large the solution asymptotes to the Euler doubly-connected rotating states, with the value of $\Omega$ scaled by a factor of $1 / 2$. In that limit, as we have already mentioned, the vorticity behaves as if it were present in both layer at once, and exact Euler behavior is recovered provided no vorticity is present in the upper and lower layer at the same horizontal position.

As an illustration of this, a set of V-states for $v=0.1$ is shown in Figure 3.10 (the vortices are in different layers, although we have drawn both of them with solid lines). Some of their properties are given in Table $3.2 a$, including $d_{c}$, the area of each vortex, its centroid $\bar{x}$, its equivalent radius $R$ and its aspect ratio $\lambda$ (obtained by fitting an ellipse to the values of the second moments of vorticity, cf. Appendix A). The interesting behavior, however, occurs as $v$ becomes negative, in which case the vortices have a nonzero overlap.

In Figure 3.11 the solutions for $v=-0.1$ are presented, and their properties are given in Table $3.2 \mathrm{~b}$. It is here that we first see the existence of a critical $\gamma$ 
beyond which no V-states exist; in particular for $v=-0.1$ we find $\gamma_{c}=11.90^{\dagger}$. Note that as $\gamma_{c}$ is approached the states become more elongated and more pointed. We are now going to show that the large curvatures, as $\gamma$ approaches $\gamma_{c}$ are the consequence the presence of a critical point in the corotating streamfunction, and that the critical point approaches the contour as $\gamma \rightarrow \gamma_{c}$.

We start with some definitions. For each $V$-state we can define a corotating frame: it is the frame that rotates with constant angular velocity $\Omega$ - the rotation rate of the $\mathrm{V}$-state in the inertial frame. Thefore, in the corotating frame, the $V$-state is stationary with respect to the coordinate axes. The streamfunction $\psi_{\text {cor }}$ in the corotating frame is related to the streamfunction $\psi$ in the inertial frame by:

$$
\psi_{\mathrm{cor}}(\mathrm{x}, \mathrm{y})=\psi(\mathrm{x}, \mathrm{y})-\frac{1}{2} \Omega\left(\mathrm{x}^{2}+\mathrm{y}^{2}\right)
$$

By equilibrium points we refer to the points in the fluid were the velocity is identically zero (so that if, for instance, a tracer particle were put at such a point, it would not move away from it). The are two quite different types of equilibrium points: centers and saddles $\ddagger$ (they are sometimes referred to as elliptic and hyperbolic points). We will try to show in the rest of this thesis, and in particular in next chapter, that the latter (often called stagnation points), which from here on we will designate by the term critical points, play a very important role in the existence, stability and evolution of isolated vortex structures. Finally we will refer to the streamlines that connect the critical points as the separatrices.

Armed with these definitions, let us try consider now the geometry of the doubly-connected rotating two-layer $V$-states. Consider first the streamfunction, in the inertial frame, of a V-state far from the limiting state. We show in Figure 3.12a, for the case $v=-0.1$ and $\gamma=2.0$, the velocity vectors on a grid large enough to encompass the whole V-state\# . Because of the symmetry of the system, we need only show one of the two layers; the other one can be obtained by reflection about the $y$-axis. Note that the velocity field is not tangent to the contour of the V-state in

\footnotetext{
IIt goes without saying that one can determine the critical value of $\gamma$ to any accuracy, provided one is willing to invest the computational resources necessary. We have decided to content ourselves with determining $\gamma_{c}$ to within 0.05 , which is of the order of a few percent for the $O(1) \gamma$ 's of interest, especially in view of our somewhat crude knowledge of a more precise location of the critical alignment distance curve.

\# This nomenclature is borrowed from the theory of analytic functions.

\# We have found this technique to be by far the easiest way to grasp the geometry of the streamfunction, since the velocities are very easily calculated from the contour positions, while the streamfunctiom connot be reduced to a simple contour integral for the case of a $\mathrm{K}_{0}$ Greens' function. Recall that the streamfunction is tangent to the velocity field at every point in the fluid.
} 
the inertial frame since the vortices are rotating at constant velocity $\Omega$ in that frame. Most importantly, however, it should be observed that the velocity field possesses only one center (located somewhere inside the contour of the V-state), and that the geometry of the streamfunction in the inertial frame consists simply of nested closed streamlines.

By contrast, as can be seen from Figure $3.12 b$, the velocity field in the corotating frame has a considerably more complicated topology. Not only two new critical points appear but two more centers are also present in that frame, for a total of five equilibrium points. In Figure 3.13 we have drawn a schematic rendition of the geometry of the corotating streamfunction that helps to bring out the salient features. The two new centers (designated $C_{1}$ and $C_{2}$ in Fig.3.13) are related to the presence of two new sets of closed streamlines that correspond to what Melander et al. (1987a) have called ghost vortices. They are a purely kinematic effect produced by the change of frame. Their presence, however, necessitates the introduction of two new critical points $\left(S_{1}\right.$ and $S_{2}$ in Fig. 3.13). It should be noted that, at this value of $\gamma$, both critical points are located at $O(1)$ distances from the contour of the $\mathrm{V}$-state.

For $\gamma=0$, the shape of the $V$-state is an exact circle and the geometry of Figure 3.13 becomes degenerate. In that case the area enclosed by the separatrices (the ghost vortices) vanishes as they coalesce into a circle on which $S_{1}$ and $S_{2}$ are also located (we will give more details about this in Chapter 4). As soon as $\gamma$ is made nonzero, keeping $v$ constant, the geometry of the corotating streamfunction becomes similar to the one shown in Figure 3.13. As $\gamma$ is increased the critical point $S_{1}$ at first approaches the contour but is eventually actually pushed away from the contour until, at a value $\gamma=\gamma_{s}$, the geometry of the corotating streamfunction changes in a dramatic fashion.

What happens is that as $\gamma$ exceeds the threshold $\gamma_{S}$ ( for the case $v=-0.1$ we find $\gamma_{\mathrm{s}} \approx 4.5$ ) the influence in each layer of the vorticity in the other layer is so strong (because the coupling constant $\gamma$ is large) that it induces a new set of closed streamlines immediately above (or below) in the other layer, and a new center and, by necessity, a new critical point are also created. In Figure 3.14 the velocity field for the limiting state $v=-0.1$ and $\gamma=\gamma_{c} \approx 11.90$ is shown, while a schematic drawing of the geometry of the corotating streamfunction for the states at $\gamma \gamma_{s}$ can be found in Figure 3.15.

The existence of a limiting $V$-state is a direct consequence of the new critical point $S_{3}$ that appears as $\gamma$ exceeds $\gamma_{s}$. Indeed, as $\gamma$ is increased further beyond $\gamma_{s}$ 
the vortex centered in $\mathrm{C}_{3}$ becomes larger and larger (because of the increased coupling between the two-layers) and pushes the critical point $S_{3}$ closer and closer to the contour of the $\mathrm{V}$-state, until, at the finite value $\gamma_{c}$, the limiting state is reached where the critical point is located on the contour itself.

To corroborate this interpretation, we have plotted in Figure 3.16 the distances $d_{i}(i=1,2,3)$, along the $x$-axis, between the critical points $S_{i}$ and the contour of the V-state as function of $\gamma$ (the numerical algorithm that was used to determine the location of the critical points is described in Appendix D). Note that $d_{3}$, which appears only for $\gamma 4.5$, vanishes at finite $\gamma \approx 11.90$, while the other two critical points remain at $O(1)$ distances from the contour of the $V$-state. In order to dispel any possible doubts as to whether $d_{3}$ actually vanishes at finite $\gamma$ or simply aymptotes to zero as $\gamma \rightarrow \infty$, we have plotted, in Figure 3.16a the values of $d_{3}$ versus $\gamma$ for the interval $11.0<\gamma<11.9$ for the $V$-states that we have determined. It is clear from that figure that $d_{3}$ is equal to zero for a finite value of $\gamma$, which can actually be obtained by extrapolating the straight line to the axis, and is found to be very close to 11.95 , the first value of $\gamma$ for which our algorithm fails converge.

The above scenario for the existence of a limiting V-state at finite $\gamma$ applies, however, only to the cases for which the amount of overlap between the two vortices (qualitatively measured by the magnitude of $v$ ) is relatively small, say $v<\tilde{v}$. The reason for this is quite simple: when the overlap becomes more considerable, the vortex in the lower (respectively upper) layer doesn't succeed in generating the ghost vortex $S_{3}$ in the upper (lower) layer because of the presence of the upper (lower) layer vortex at that same location. It turns out that for $v$ sufficiently large the geometry of the corotating streamfunction is always qualitatively similar to the one of Figure 3.13, namely with only two critical points present.

To illustrate this behavior we show, in Figure 3.17, a set of states at $v=-0.7$ for which $\gamma_{c} \approx 3.55$ (the properties are found in Table 3.2c). The limiting state comes about because the critical point $S_{2}$ (cf. Figure 3.13) touches the contour of the $V$ state at $\gamma=\gamma_{c}$. It is interesting to point out that, at this larger value of $v$, both critical points approach the contour monotonically as $\gamma$ is increased from zero, and, as we have said, there is no transition in behavior at some $\gamma=\gamma_{\mathrm{s}}$.

Consider the corotating velocity field for the limiting state for $v=-0.7$ at $\gamma_{c} \approx 3.55$ shown in Figure 3.18. Notice that the critical point $S_{2}$ is almost coincident with the contour and that the amount of overlap between the two states is very large (cf. Figure 3.18 for this). To see how the critical points move as $\gamma$ is varied, we have plotted the distances $d_{1}$ and $d_{2}$ between the critical points $\left(S_{1}\right.$ and $S_{2}$ respectively) 
and the contour of the $\mathrm{V}$-state as a function of $\gamma$, in Figure 3.19. Note the monotonic character of these functions, in contrast with the richer behavior at smaller values of $v$ (cf. Figure 3.16). In Figure 3.19a the values of $d_{2} v s \gamma$ for the last six V-states are shown to fall on a straight line, leaving no doubt as to the fact that $d_{2}$ vanishes for a finite value of $\gamma$.

It is perhaps remarkable that, at high $\gamma^{\dagger}$, the shapes for two vortices that are almost overlapping are so different from circular. Why this is so can be understood, in qualitative sense, from the fact that as the coupling between the two layers increases each vortex tends to become elongated in order to resist the shear induced by the other vortex and this, as we will have the opportunity to show in detail in the next chapter, brings the critical points of the corotating streamfunction closer to the contours so that, at the finite value of $\gamma=\gamma_{c}$, the critical points coincide with the contour and for $\gamma \gamma_{c}$ no solution exists $¥$.

We conclude this section by turning to the difficult question of determining whether the value $\gamma_{c}$ at which convergence ceases corresponds to the value $\gamma_{e}$ at which the solutions stop existing. Clearly when we can show that the distance between the contour of the $\mathrm{V}$-state and a critical point vanishes for a finite value of $\gamma$ (as for instance in Figure 3.16a or 3.19a) were are left with little doubt that the failure of the algorithm to converge beyond $\gamma_{c}$ is truly associated with the fact that states simply do not exist beyond that value of $\gamma$.

It however turns out that, for some values of $v$ not far from $\tilde{v}$, where the transition in behavior occurs\#, the cessation of convergence at $\gamma_{c}$ cannot be associated with a critical point becoming extremely close to the contour. One such example is given in Figure 3.20, where the $V$-states for $v=-0.20$ are shown (the properties are given in Table 3.2d). Notice how the last very elongated states near

\footnotetext{
t We would like to point out that, from a quantitative perspective, "large $\gamma$ " usually means between $1<\gamma<5$. This is a consequence of the exponentially asymptotic behavior of the modified Bessel function of order zero.

₹ The linear theory of Flierl (1988 and priv. comm.) seems to indicate that finite amplitude $m=1$ perturbations to a barotropic circular vortex equilibrate at all values of $\gamma$ with the exception of one at which a resonance seems to occur. It is therefore quite probable that, at a much larger value of $\gamma, V$-states of this sort do exist. They would however have quite different shape from the one presented here, in particular they would be elongated in the opposite direction. We have been unable, numerically, to determine their existence; however their relevance to the alignment problem at the values of $\gamma$ we have investigated with initial value calculations is probably very weak, and their stability would still have to be ascertained even if they were found to exist.

\# Our knowledge of the critical value $\nabla$, at which the behavior changes from the one indicated of Figure 3.11 to the one of Figure 3.17, is somewhat crude because we have determined the solutions for only 9 values of $v$ (cf. Table 3.1). Our results indicate that $-0.1>\nabla>-0.15$.
} 
$\gamma_{c}$ show little evidence of a cusp developing. An analysis of the critical points shows in fact that even near $\gamma_{c}$ their distance to the contour is of $O(1)$.

The interesting question to be resolved here is whether it is possible to find a limiting $\mathrm{V}$-state (according to the definition given above) whose contour does not contain a singularity. This would be a novelty since all known examples of singlecontour V-states (for the Euler equations) are known to possess limiting states that are associated with the development of infinite curvatures on the contours (associated with a critical point) $\dagger$. Faced with the failure of our algorithm to converge for $\gamma \gamma_{c}$ without the presence of a critical point "very near" the contour, we are thus confronted with two possibilities: either $\gamma_{c}=\gamma_{e}$, i.e. the state at $\gamma_{c}$ is truly a limiting state although it is smooth, or else our algorithm is simply not powerful enough to be able to converge for the states beyond $\gamma_{c}$ (which however exist).

We have first considered the latter eventuality, and have thus implemented a number of simple strategies to improve our power of convergence without major changes to the algorithm. As is described in more detail in appendices these have included:

a) increasing the number of points and distributing them in greater amounts in the regions of high curvature (cf. Appendix B).

b) decreasing the increment in $\gamma$ between two successive states to avoid the possibility that convergence fails due to the choice of an initial guess for the iteration that is to "far" from the actual solution.

c) using a logarithmically desingularized algorithm (cf. Appendix C) Although these improvements have allowed us to find $V$-states a very small amount beyond $\gamma_{c}$, the qualitative result remains that convergence fails when the contours show no evidence of points of infinite curvature developing. We thus propose that, if indeed the failure of the algorithm to converge must be attributed to its inadequacy (as opposed to the non-existence of solutions), a first step could be taken by increasing its order, as, for instance, Wu et al. (1984) have done to accurately resolve the cuspy limiting Euler V-states. We note, however, that the present algorithm is able to converge quite easily into very cuspy states (cf. for instance Figure 3.17), and we suspect that the difficulty here (if indeed there is one) is due to the "high" values of $\gamma$ that induce the function $K_{0}$ to behave very differently from a logarithm.

† Dritschel (1988c) has recently presented Euler V-states with nested contours (corresponding to different vorticity levels) that also appear to exhibit the existence of "smooth" limiting states. 
The other hypothesis that we have investigated is that the failure to converge must be attributed to the factual non-existence of solutions beyond $\gamma_{c}$. If that were the case, one ought to be able to detect in the behavior of the solutions at $\gamma<\gamma_{c}$ some indication that the states will cease to exist for $\gamma \gamma_{c}$. In the previous examples known of limiting states the shape itself of the solutions, by becoming nondifferentiable, signals the approach of a region of parameter space were the solutions fail to exist.

As we have said (cf. Figure 3.20), the shape of the contours for some twolayer doubly-connected rotating $\mathrm{V}$-states shows no sign of an approching limiting state near $\gamma_{c}$. One possibility we have explored $\$$ is that at $\gamma=\gamma_{c}$ we may be in the presence of a fold in the solution as illustrated by Figure 3.21, where $A$ designates some quantity (amplitude) that represents a V-state (say its area, or aspect ratio, etc.). If the solutions in the $(A, \gamma)$ plane happened to fall on a curve that had an infinite slope (when $\gamma$ were taken to be the independent variable) at $\gamma_{c}$, one would of course be unable to find solutions for $\gamma \gamma_{c}$. However, since the solution curve does not terminate at $\gamma_{c}$, but simply "folds back", no catastrophe occurs at $\gamma_{c}$ and if A were made the independent variable one would easily be able to find the solutions above $A_{c}$ by marching along the solution curve by incrementing $A$ instead of $\gamma$.

With our algorithm we cannot afford to fix quantities like $A$ throughout the iteration but, if we were in the presence of the above scenario, we ought to be able to detect a incipient infinite slope near $\gamma_{c}$ as these quantities are plotted against $\gamma$ for the solutions obtained at $\gamma<\gamma_{c}$. Unfortunately, we have been unable to do so for any of the quantities in Table 3.2d, which represent, at least, the few most obvious properties of the solution. The examination of these quantities versus $\gamma$ shows no indication of a fold in the solution of the type shown in Figure 3.21.

In conclusion we confess that, in spite of our efforts, we have at present been unable to settle this issue, and we suspect that a considerable amount of work is going to be necessary to decide whether the smooth $V$-states at $\gamma_{c}$ are true limiting states or simply location in parameter space where convergence becomes a lot more difficult to achieve. The same considerations apply to some two-layer dipolar V-states that we present next.

$\mp$ We owe this suggestion to Prof. Joe Keller. 


\subsection{Two-Layer Dipoles}

We turn now the study of dipolar coherent vortex structures within the twolayer quasigeostrophic model. These have been the subject of a number of investigations in recent years that it will prove useful to review before proceeding to present our own results.

Two main approaches must be distinguished. On one side, the analytical approach has yielded a variety of exact, though discontinuous, solutions for pairs of vortices in a baroclinic fluid usually referred to as modons (Flierl et al. 1980). These are essentially the extension of the Batchelor solution for the Euler equation (Batchelor 1967, pp.534-535) to the quasi-geostrophic two-layer model. These solutions are $\mathrm{C}^{(2)}$ in the streamfunctiont, i.e. discontinous in the first derivative of the vorticity; and can be extended to include the effects of a planetary gradient of vorticity (the $\beta$-plane) provided their propagation is such that no Rossby radiation is induced by their motion through the fluid.

The state of our knowledge as to the stability of these solutions is not very advanced. However numerical simulations (McWiliams et al. 1981) seem to suggest that barotropic modons may be unstable to perturbations of moderate amplitude. There is also some evidence (Flierl, priv. comm.) that baroclinic modons may be unstable in a similar way.

On the other side, we must recall the point vortex work of Hogg and Stommel (1985a and b) and Young (1985). They have studied in some detail, within the context of two-layer quasigeostrophy, the interactions of propagating pairs of point vortices of opposite sign, one vortex in each layer, to which they assigned the name hetons $\ddagger$. Because of their simplicity, these point vortex solutions exhibit extremely aesthetically pleasing behavior of great variety as they interact. The solutions we are going to present next can be considered to be the desingularized extension of these point vortex pairs (we are unfavourable to the term "finite area hetons").

It should be recalled that quasigeostrophic two-layer finite-area constantvorticity dipoles have already been observed to emerge naturally as the end

$\dagger$ Recall that the finite-area constant vorticity solution obtained via contour dynamics are only $C^{(1)}$, i.e. only the velocity field is continuous throughout the fluid.

$\mp$ We note, incidentally, that point vortex dipolar solutions have also been found on the $\beta$-plane (Zabusky and McWilliams 1982) and in a continuously stratified quasigeostrophic situation (Flierl 1987). 
product of the decay of a perturbed baroclinic vortex (Helfrich and Send 1988). This problem was originally proposed by Pedlosky (1985), who considered the instability of a circular vortex in the upper layer, located directly above a circular vortex of equal but opposite vorticity in the lower layer; he studied both the linear and weakly non-linear problem (the same questions were more recently revisited by Flierl 1988).

Helfrich and Send (1988) performed inital value contour dynamical simulations to determine the large amplitude behavior of the perturbed vortex, and confirmed that, following the simple arguments of Pedlosky (1985), an unstable perturbation of mode $m$ (the quantized angular wavenumber) will lead to the breakup of the vortex into $m$ two-layer dipoles. To illustrate this point, we have reproduced in Figure 3.22 (from Helfrich and Send), a beautiful example of a perturbed baroclinic vortex breaking into 3 two-layer dipoles.

We are now ready to present the result we have obtained for the stationary translating vorticity configurations in two-layers, of which a sketch is shown in Figure 3.23. Note that the vorticity has opposite sign in the different layers (this is necessary for propagation). Since we have confined ourselves to the case of two equal layers $(\delta=1)$ only two parameters are left to be chosen: $\gamma$ and the ratio $\mu=x_{A} / x_{B}$ of the inner to outer radius. The question we are addressing is essentially identical to the one we asked in the preceding section: for what values of $\gamma$ and $\mu$ can we find dipolar V-states?

As we have done throughout, we have investigated the matter by increasing $\gamma$ gradually from zero for a fixed value of $\mu$. In contrast with the one-layer dipoles, and in analogy with the two-layer doubly connected rotating $V$-states, the interesting results in this problem are found for negative values of $\mu$, i.e. when the two vortices have a nonzero overlap. For completeness' sake, however, we start by consider the simple $\mu>0$ case (details of the algorithm are given in Appendix B2).

In Figure 3.24 we show a set of solutions for $\mu=0.05$; their properties are given in Table 3.3a. In particular we have tabulated the velocity and its ratio to the velocity of an equivalent point vortex hetont, the area of each vortex and its ratio to

t We must warn the reader that this comparison is unfortunately of little value unless $\gamma$ is small. From (1.4) it is easy to see that, for a $V$-state of area $A$ and intercentroid distance $d_{c}$, the most reasonable choice for the velocity of an equivalent point vortex heton is:

$$
V_{p v}=\frac{s}{4 \pi}\left[\frac{1}{d_{c}}-\sqrt{2} \gamma K_{1}\left(\sqrt{2} \gamma d_{c}\right)\right]
$$

with the strength $s=A$ (recall that, without loss of generality, we have chosen the vorticity identically equal to 1 ). What needs to be kept in mind is that the choice $s=A$ is not a necessary one. Indeed, while for an Euler circular 
the circular solution at $\gamma=0$, the intercentroid distance, the aspect ratio $\lambda$ of each vortex (here $0<\lambda<1$, and $\lambda=1$ corresponds to a circle) and its equivalent radius $R$. In perfect analogy to the $V$-states of the precending section, as $\gamma$ is increased from zero the circular shapes become more elongated and as $\gamma \rightarrow \infty$ the Euler dipolar solutions of Deem and Zabusky (1978a and b) are recovered, with the velocity reduced by a factor of 2 , as can be seen directly from (1.4).

The interesting behavior starts to appear as $\mu$ is made negative. The case $\mu=-0.1$ is shown in Figure 3.25 (and Table 3.3b exhibit the properties of these states). In a way not dissimilar with what we have shown occurs for the rotating twolayer $\mathrm{V}$-states, our algorithm fails to converge beyond some critical value of $\gamma$. For $\mu=-0.1$ we find $\gamma_{c} \approx 2.95$ (we have limited our knowledge of $\gamma_{c}$ to only \pm 0.05 ). Here also we have attempted a series of simple improvements to the algorithm in order to improve the convergence, as was explained in the previous section, but without much success.

A consideration of the cotranslating streamfunction, for which a sketch is provided in Figure 3.26, suggests that the failure to converge may be due to the presence of the critical points $S_{1}$ and $S_{2}$ that approach the contour as $\gamma$ is increased, which is the reason for the presence of a limiting state for the dipolar $V$ states of the Euler equation (cf. Wu et al. 1984, for details). However, from the smoothness of the contours at $\gamma_{c}$, it is difficult to imagine that would be the case.

We illustrate this point in detail for the case $\mu=-0.3$, for which the $V$-states are shown in Figure 3.27 (cf. also Table 3.3c) and where $\gamma_{c} \approx 2.20$. The minimum distance $d_{\min }$ from the critical point $S_{1}$ to the contour is plotted versus $\gamma$ in Figure 3.28. Although it decreases with increasing $\gamma$, it still has $O(1)$ values when convergence is lost (i.e. at $\gamma_{c}$ ), and no signs of an infinite slope of this quantity versus $\gamma$, which, as was explained in the previous section, may suggest the approach to a limiting state, can be detected near $\gamma_{c}$. It thus remains unclear whether the state at $\gamma_{c}$ is a smooth limiting state.

vortex of radius 1 the exterior velocity field is identical to one of a point vortex of strength $\pi$, such is not the case when the Greens ${ }^{*}$ function is of modified Bessel type. Thus, for instance, it is easy to show that the strenght $s_{e q}$ of the point vortex that produces a field identical to the exterior of an equivalent barotropic circular vortex is given by:

which, of course, reduces to $\pi$ in the limit $\gamma<1$.

$$
\mathrm{s}_{\mathrm{eq}}=\pi\left[\frac{2 \mathrm{I}_{1}(\gamma)}{\gamma}\right]
$$

$¥$ Throughout the remainder of this section we only plot, for the sake of clarity, the vortex in the upper layer. 
Similarly to the results of the previous section, we find that as the overlap becomes larger, i.e. as $\mu$ becomes more negative, the behavior changes drastically beyond the critical value $\tilde{\mu}$. A new scenario appears at $\mu=-0.6$ for which the $V$. states are shown in Figure 3.29 (and the properties given in Table 3.3d).

As $\gamma$ is increased from zero the vortices at first simply elongate in the familiar way to maintain a stationary configuration in the presence of the shear due to the other vortex, and, in the cotranslating frame, the geometry of the streamfunction is identical to the one illustrated in Figure 3.26, i.e. with two centers and two critical points. However, when $\gamma$ becomes large enough, the coupling is so strong that the vortex in each layer is capable of producing two new sets of closed streamlines, thereby adding two centers and, by necessity, one new critical point.

This can be seen by considering the velocity field, for example, of the state at $\mu=-0.6$ and $\gamma=2.90$ which is given in Figure 3.30. The two new centers and a new saddle point $\left(S_{0}\right)$ on the $x$-axis appear, as the vorticity in each layer seems to withdraw from the strong influence of the vorticity in the opposite layer and we find that, at even larger $\mu$, it is the new critical point $S_{0}$ which becomes responsible for the existence of a limiting $V$-state at finite $\gamma$.

This can be seen more clearly from the states shown in Figure 3.31 at $\mu=-0.9$ (see Table $3.3 e$ for their properties) where the algorithm happens to converge well enough to show us the beginning of the development of a cusp (point of infinite curvature) on the contour as the critical pont $S_{0}$ approaches the boundary when $\gamma \rightarrow \gamma_{c} \approx 2.50$. The distance between the critical point $S_{0}$ and the contour is plotted versus $\gamma$ in Figure 3.32 for the last few values of $\gamma$. From an extrapolation of the points, it is reasonable to believe that, for this value of $\mu$, there exist a limiting $V$-state due to the approach of critical point $S_{0}$.

We would like to point out, for the distracted reader who may not have noticed it yet, that, at large $\gamma$ and $\mu$ not too far from -1 , the centroid of each vortex has crossed the $y$-axis with respect to its location at $\gamma<<1$, so that the velocity has also changed sign. This simple statement implies that there exists a $V$-state, say at $\gamma=\gamma_{0}$, for which $V=0$, i.e that does not translate. Thus, for instance, for the case $\mu=-$ 0.9 we find $2.12<\gamma_{0}<2.15$ (cf. Table 3.3e). As can be seen from Figure 3.31, this state can be considered as the finite amplitude extension of a mode 1 plus a mode 3 perturbation on a circular baroclinic vortex whose linear stability was originally considered by Pedlosky (1985). In his recent study, Flierl (1988) using an asymptotic expansion in small amplitude, has predicted the existence of nonlinear equilibrated solutions to the perturbed baroclinic vortex (see also Helfrich and 
Send for computational evidence for the existence of this type of solutions). We believe that the $V$-states at $\gamma=\gamma_{0}$ are the first example of finite amplitude solutions that confirm the (vanishing amplitude) results of Flierl (1988).

More than the actual reversal in the direction of propagation, the shapes themselves of the states near $\gamma_{c}$ are so different from the ones at $\gamma<<1 \dagger$ that one may legitimately be concerned as to whether our particular method of solution is such that, without being aware of it, we have started out on one branch of finite amplitude solutions (at $\gamma<<1$ ) and ended up on a different branch at $\gamma \approx \gamma_{c}$. As a general rule, to increase the efficiency of the computation, we have used as a first guess for the iteration at the each value of $\gamma$ the converged $V$-state at the previous value of $\gamma$ (recall that $\mu$, the ratio of inner to outer radius, is the same for a whole set of states). For the first value of $\gamma$ we know that the solution has a nearly circular shape, and we thus use an exact circle as the seed for the iteration.

The question we are addressing is the sensitivity of the results just presented to the choice of the initial guess for the iterative scheme. In particular, one may wonder whether the somewhat peculiar looking shapes at $\gamma \approx \gamma_{c}$ aren't the result of the procedure delineated above. To corroborate our result we have calculated, as an example, the set of $V$-states for $\mu=-0.9$ using as the initial guess a perfectly circular shape for each value of $\gamma$. The results for this set of states, whose shape are indistinguishable from the ones of Figure 3.31, are presented in Table $3.4 \mathrm{~b}$ (for the reader's convenience we have retabulated the results of Table $3.3 \mathrm{e}$ in Table 3.4a, with, in addition, the number of iterations necessary to achieve convergence at each value of $\gamma$ ).

Note that the differences are only in the fifth decimal figure, which is probably unreliable due to the moderate accuracy with which the V-state were computed (100 nodes per vortex). Of course, as can be seen in Tables $3.4 a$ and $b$, when the iteration for each V-state is inititialized with a circle the number of iterations necessary to achieve convergence is considerably larger than when the $\mathrm{V}$-state at the previous value of $\gamma$ is used as the initial guess.

What we can conclude from the fact that, even with a circular initial guess at all $\gamma$, our algorithm converges to the solutions of Figure 3.31 is that if there exist, for the same value of $\mu$, a new branch of solutions, their shapes would be very

\footnotetext{
† At $\gamma<<1$ one would guess that the dominant modes are 1 and 2, while, from a simple inspection of the figures, if seems more likely that the dominant modes are 1 and 3 for $\gamma$ near $\gamma_{c}$.
} 
different from the ones presented here, although we cannot at this point exclude their existence.

We hope to have given enough evidence throughout this chapter to support the claim that a careful study of the topology of the corotating or cotransiating fields yields much insight into the question of existence of stationary states of vorticity. Extending this idea from a static to a dynamic context, we will show, in the next chapter, how the critical points of the corotating streamfunction play an even more crucial role in the evolution of unsteady voticity configurations. 
[This page has been accidentally (!) left blank] 


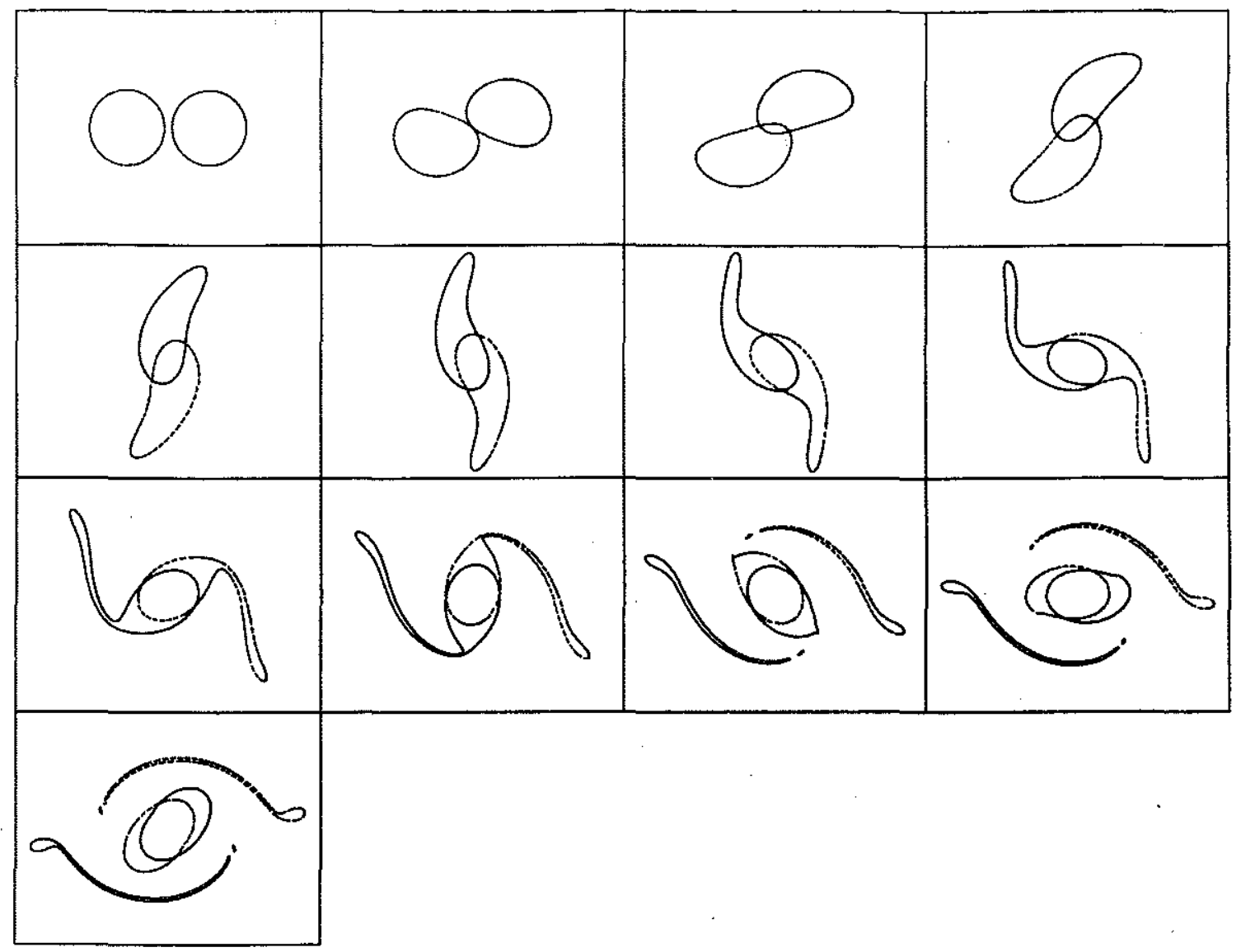

FIGURE 3.1. Alignment at $\gamma=1.25$ for $d_{c}=2.2$ at $t=0$. The solid (dashed) line represents the upper (lower) layer contour. Time increases to the left and downwards. The frames shown correspond to $t=0,4,12,16$, etc.

FIGURE 3.1a. The inter-centroid distance versus time for the above run. The discontinuity at $t \approx 36$ is an artifact of the way in which the distance is calculated (see text).

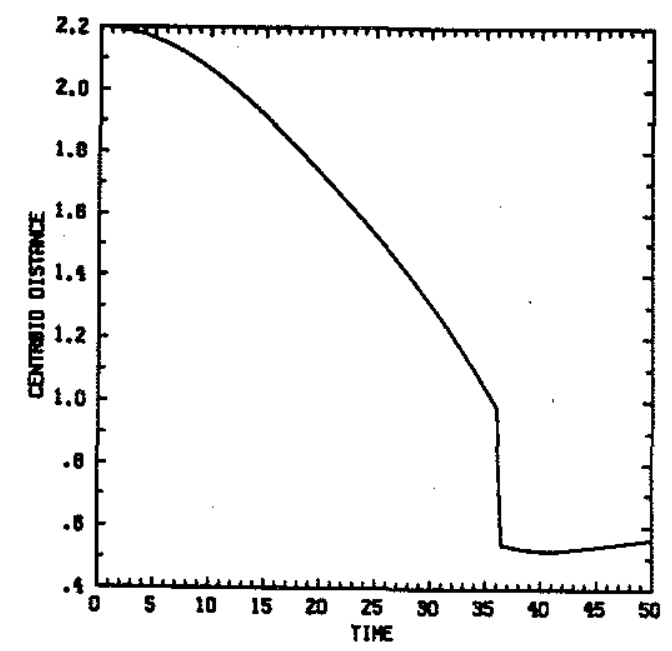




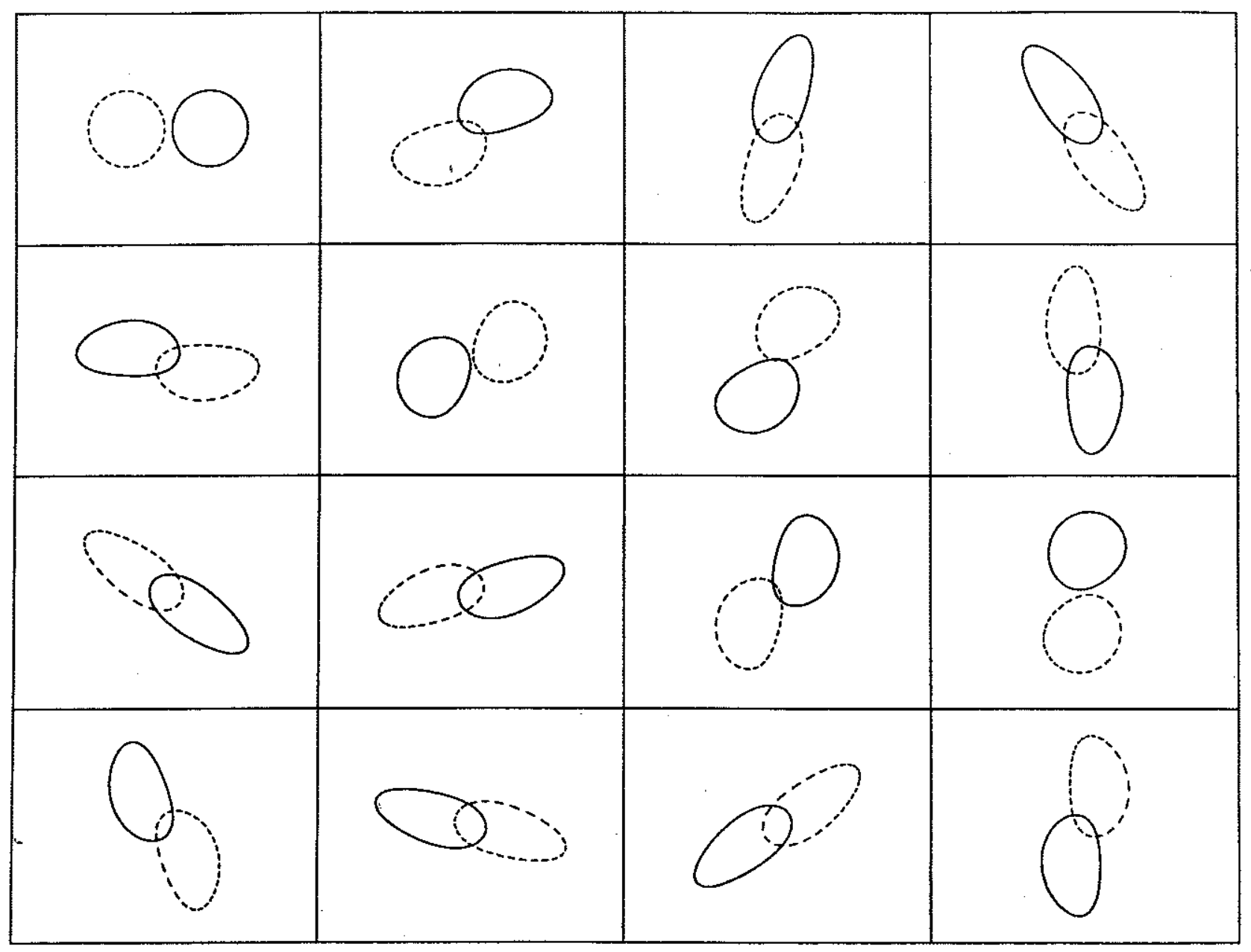

FIGURE 3.2. Alignment at $\gamma=1$ for $d_{c}=2.2$ at $t=0$. The frames are at $t=0,8,16,24$, etc.

FIGURE 3.2a. The inter-centroid distance versus time for the above run.

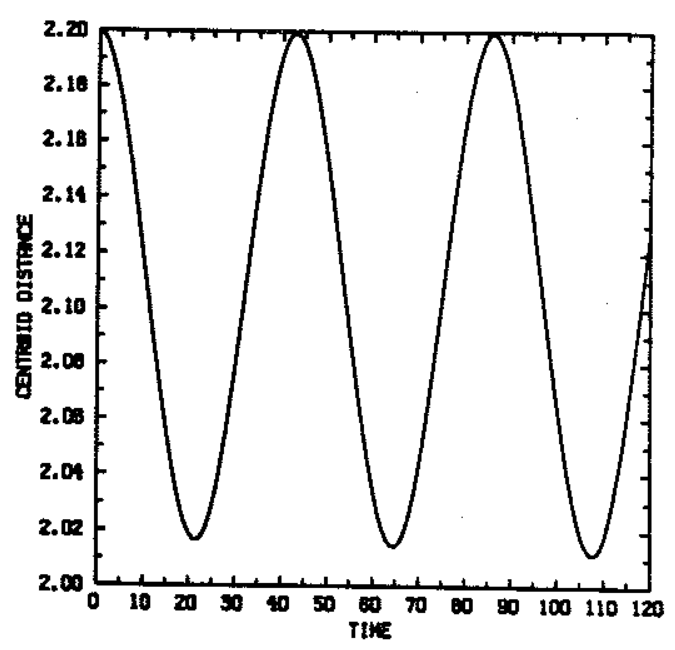




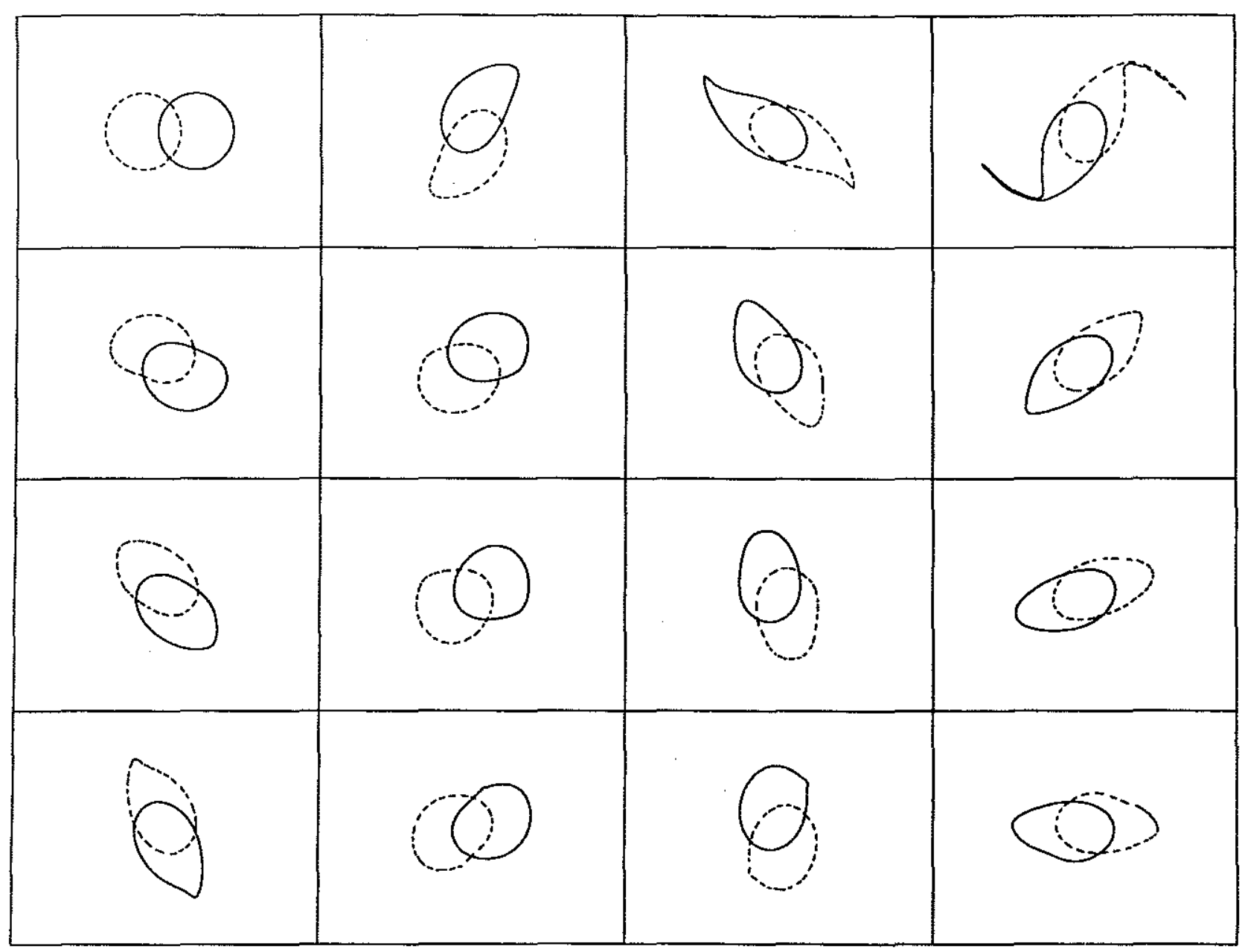

FIGURE 3.3. Alignment at $\gamma=1$ for $d_{c}=1.4$ at $t=0$. The frames are at $t=0,10,20,30$, etc.

FIGURE 3.3a. The inter-centroid distance versus time for the above run.

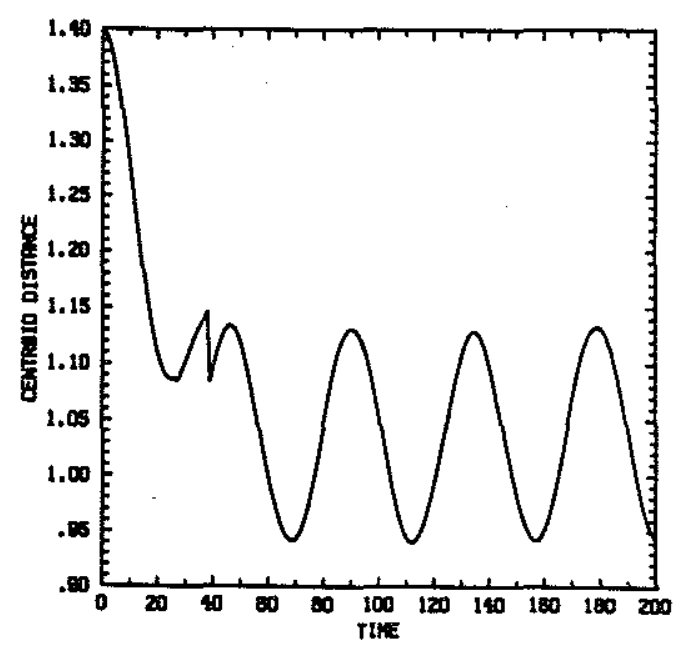




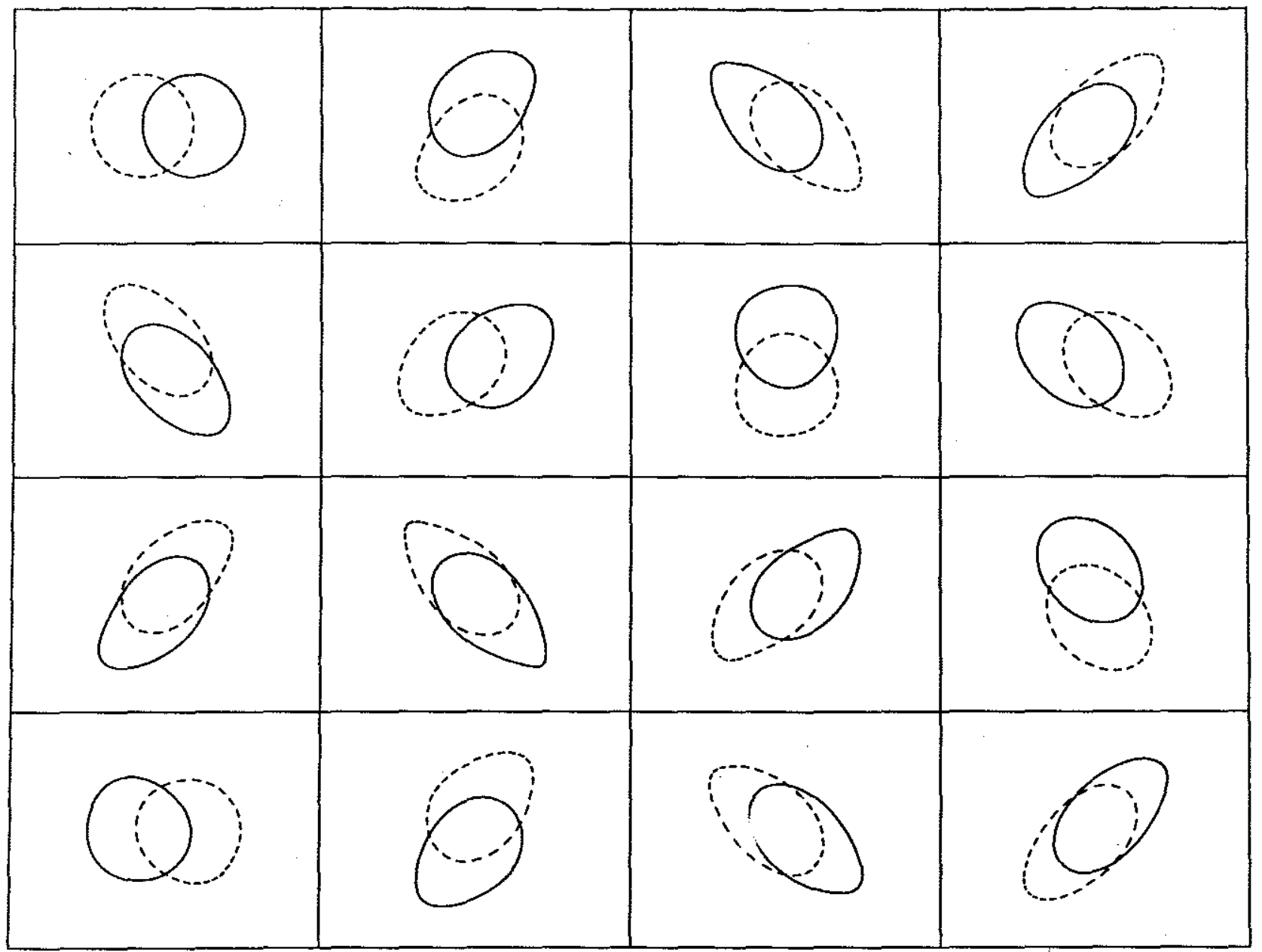

FIGURE 3.4. Alignment at $\gamma=1$ for $d_{c}=1$ at $t=0$. The frames are at $t=0,8,16,24$, etc.

FIGURE 3.4a. The inter-centroid distance versus time for the above run.

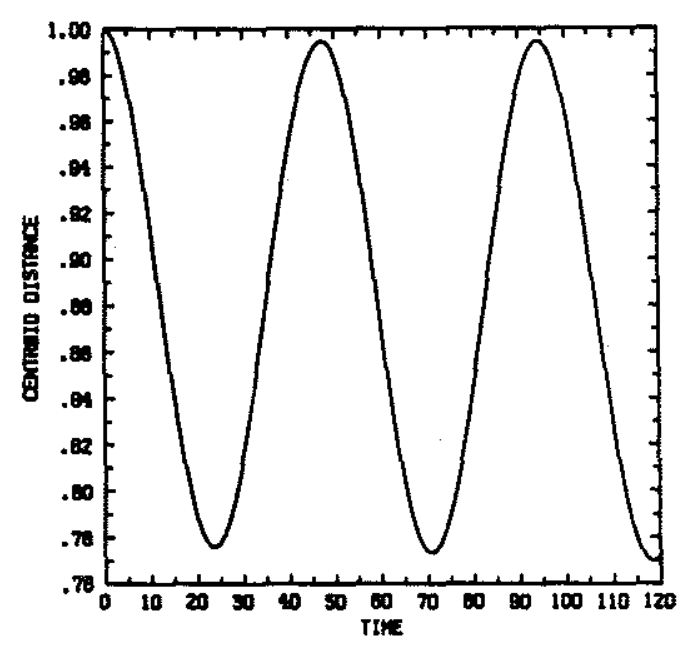




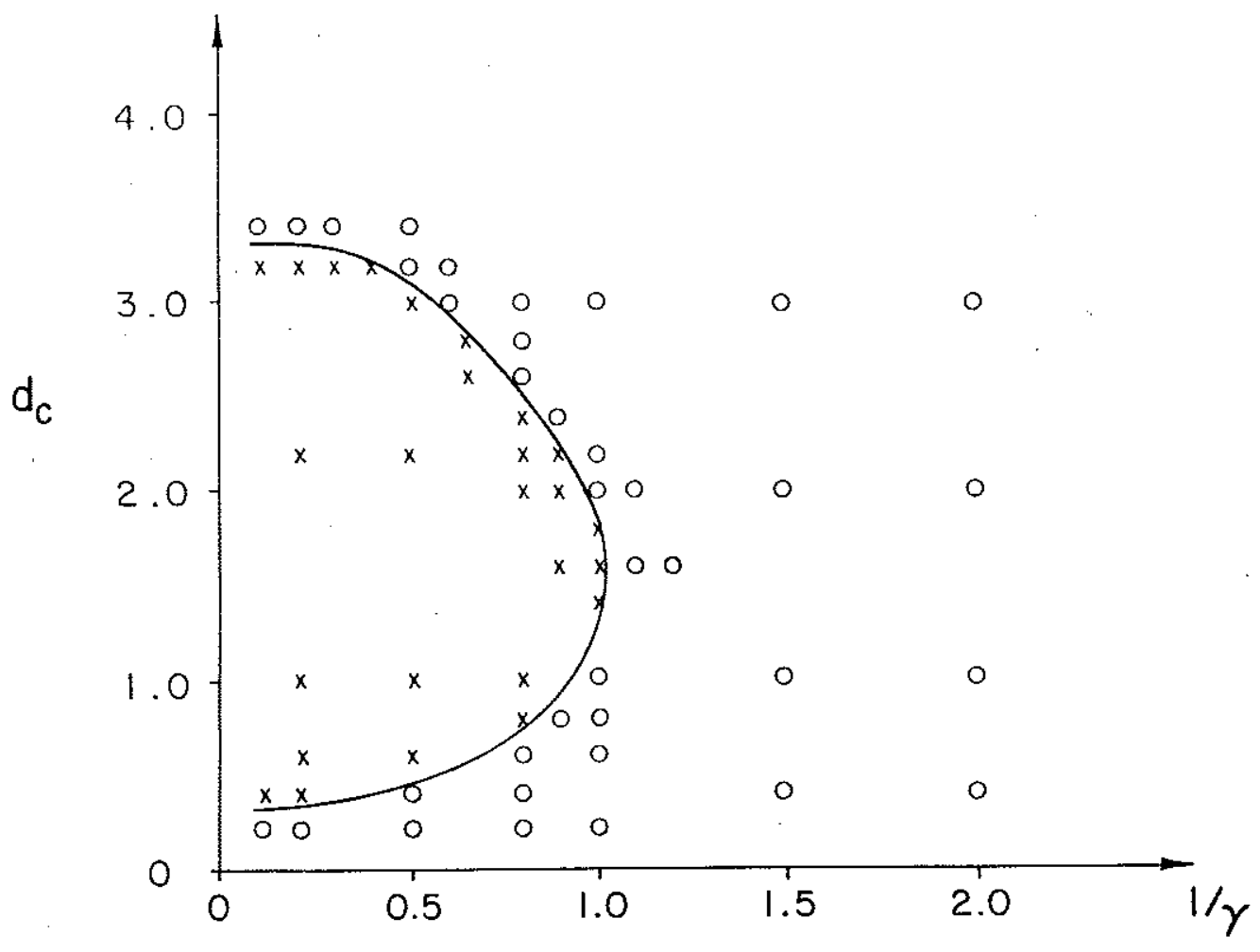

FIGURE 3.5. Phenomenology of the alignment problem in two-layer quasigeostrohy. Crosses indicate alignment, and circles no-alignment. 


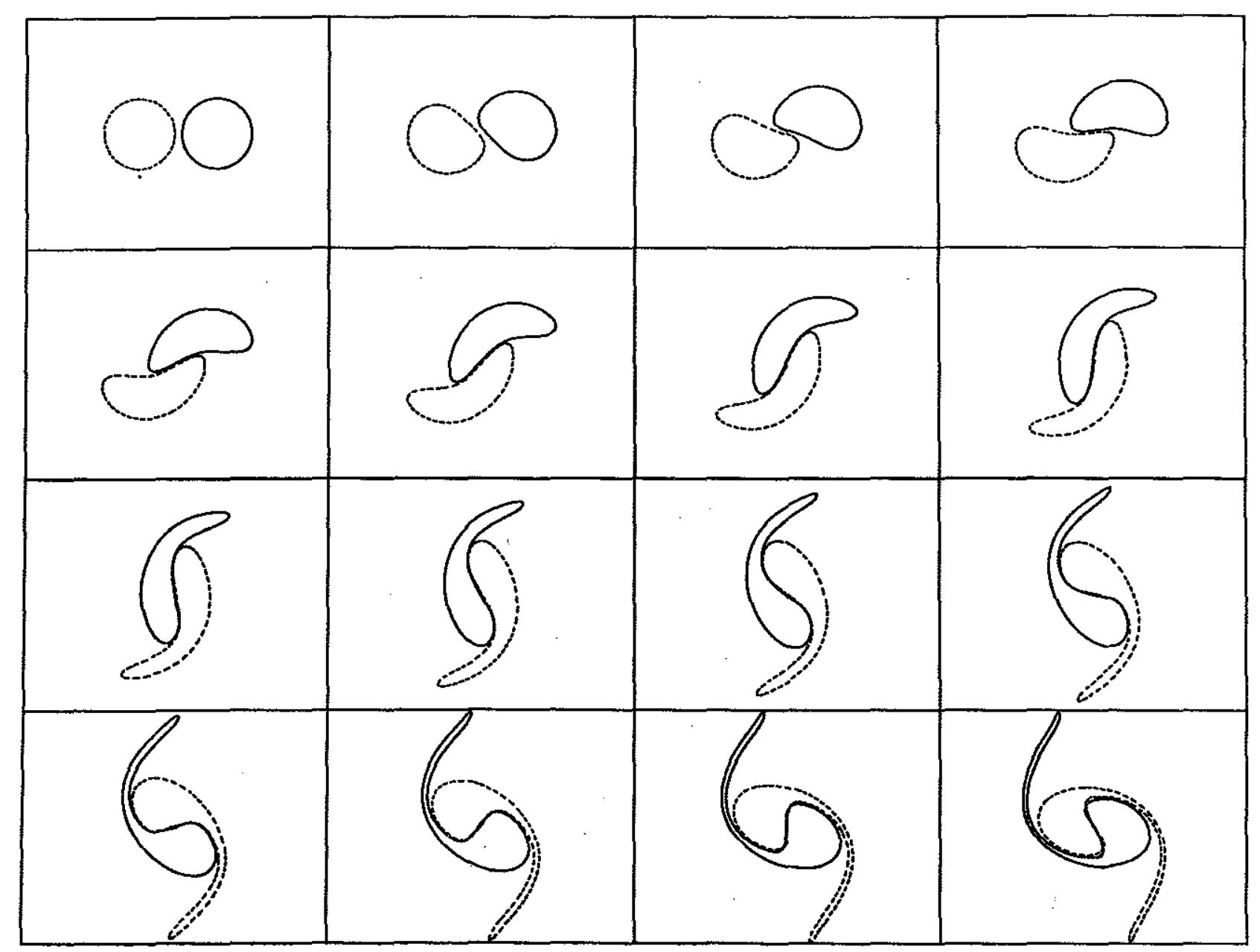

FIGURE 3.6. Alignment at $\gamma=5.0$ for $d_{c}=2.2$ at $t=0$. The frames are at $t=0,2,4,6$, etc. 


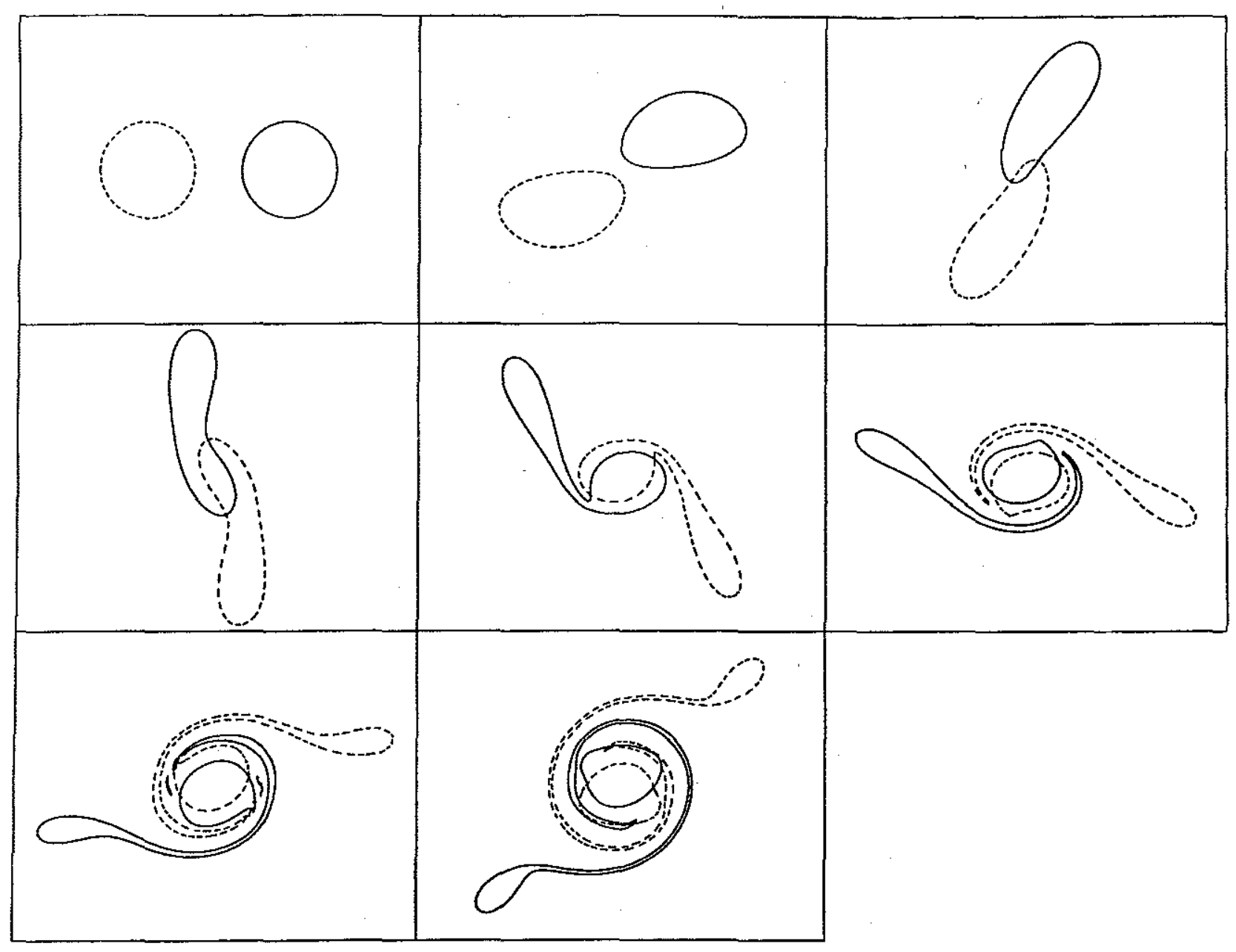

FIGURE 3.7. Alignment at $\gamma=2.0$ for $d_{c}=3.0$ at $t=0$. The frames are at $t=0,10,20$, etc. 


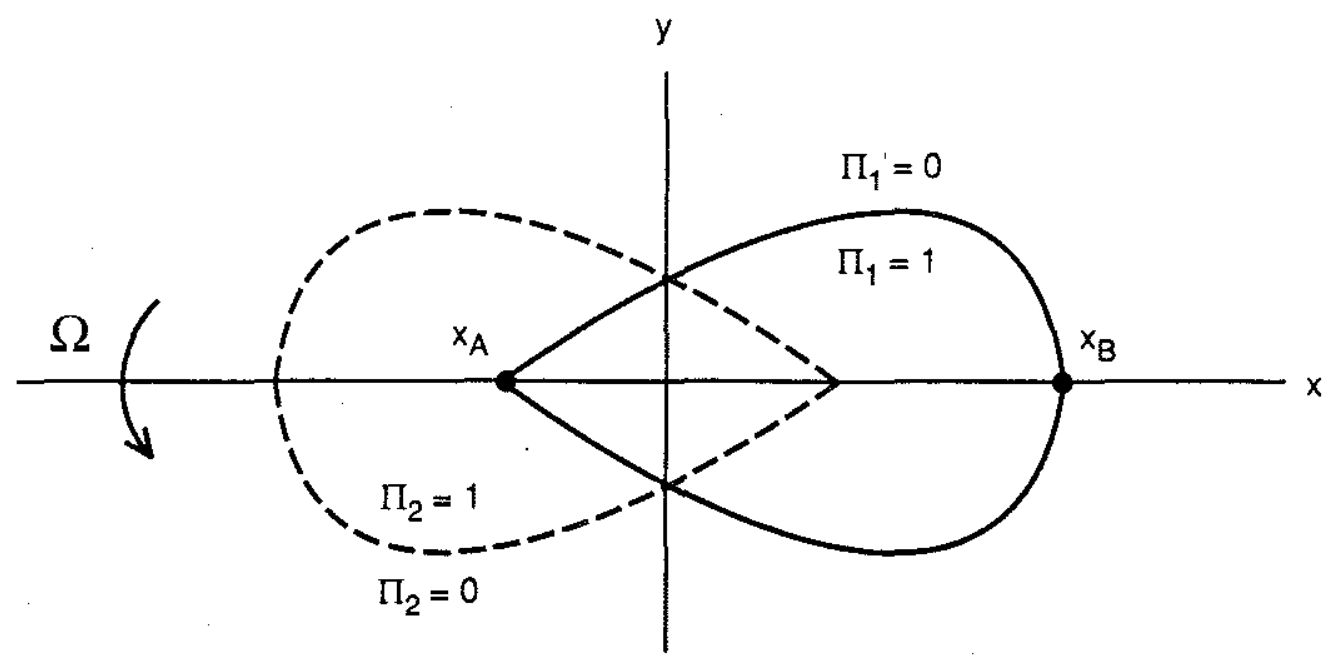

FIGURE 3.8. Schematic drawing of a two-layer doubly connected rotating V-state. The solid (dashed) vortex is in the upper (lower) layer. $\left(v \equiv x_{A} / x_{B}\right)$. 
Table 3.1

$\begin{array}{rcc}\mathrm{x}_{\mathrm{A}} & \gamma_{\mathrm{c}} & \mathrm{d}_{\mathrm{c}} \\ -0.10 & & \\ -0.15 & 4.02 & 3.23 \\ -0.20 & 1.29 & 2.00 \\ -0.30 & 1.48 & 2.50 \\ -0.40 & 1.23 & 1.34 \\ -0.50 & 1.30 & 0.95 \\ -0.60 & 1.44 & 0.66 \\ -0.70 & 1.63 & 0.46 \\ -0.80 & 1.92 & 0.29 \\ & 2.45 & 0.16\end{array}$

Chapter 3 


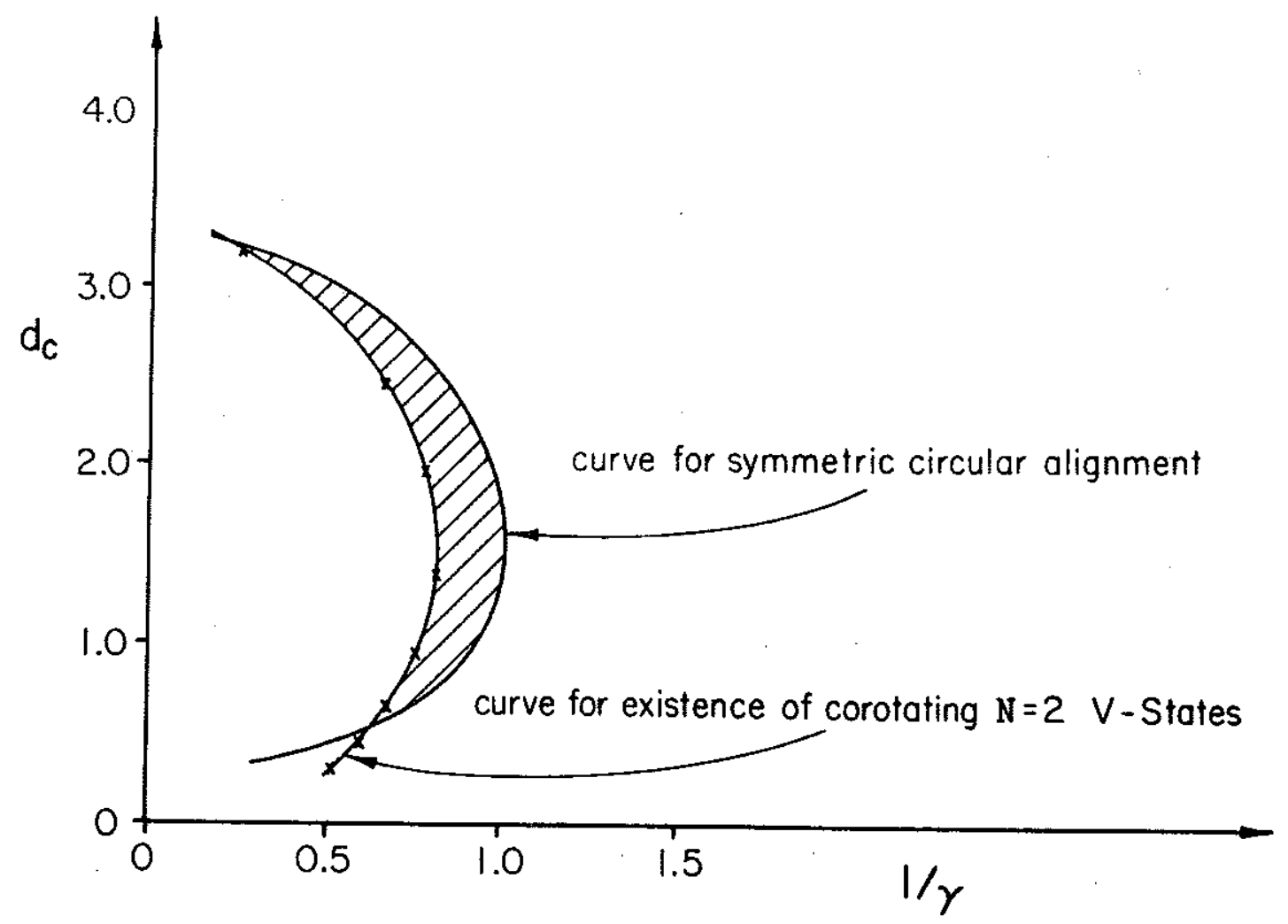

FIGURE 3.9. Alignment and the existence of doubly-connected corotating V-states. 


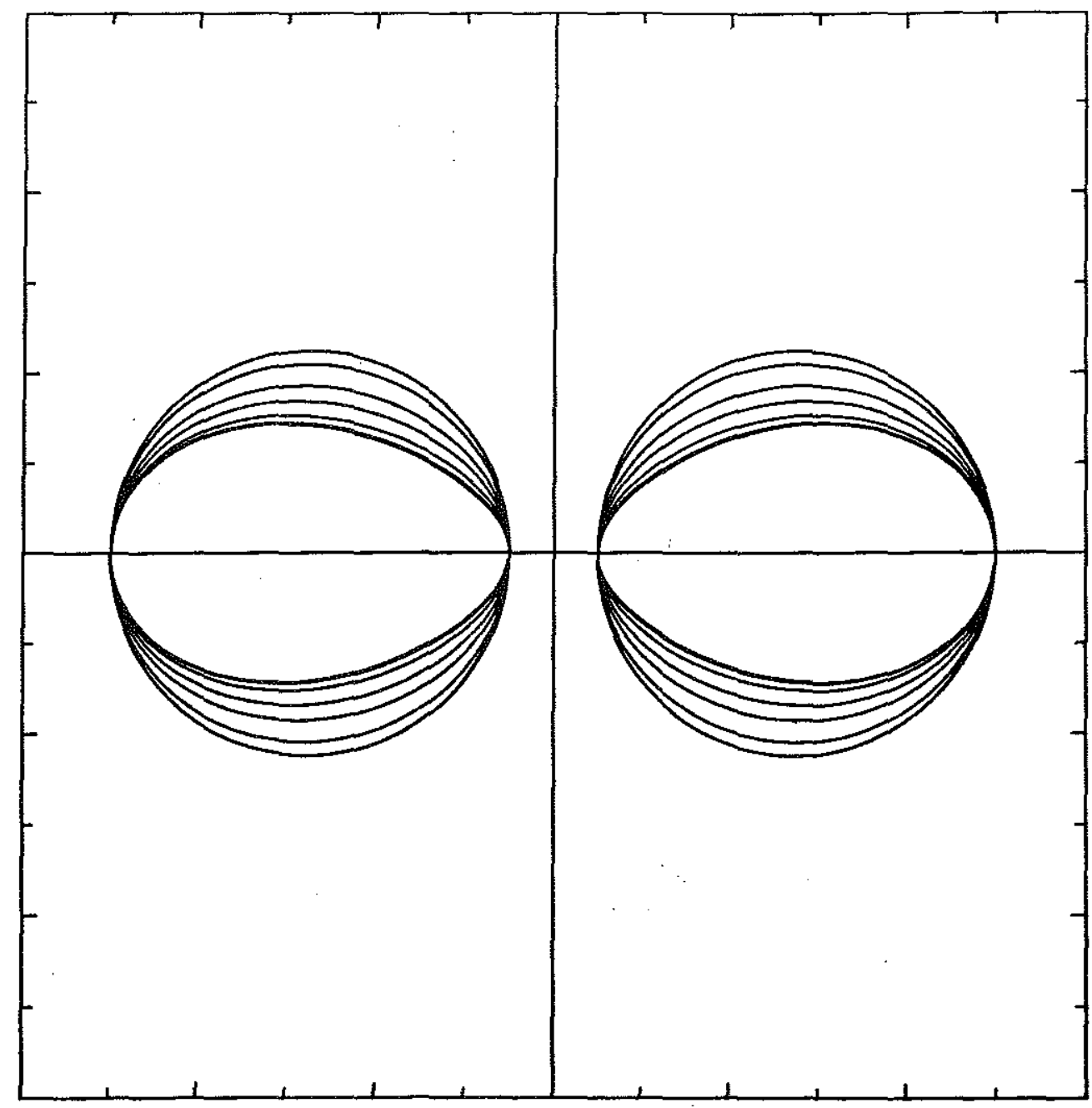

FIGURE 3.10. Doubly-connected two-layer corotating $V$-states for $v=0.1$. The values of $\gamma$ are given in the table below. As $\gamma$ increases the states become more elliptical.

Table $3.2 \mathrm{a}$

$$
\text { nu }=0.1
$$

$\begin{array}{rcccccc}\text { Gamma } & \text { Omega } & \text { dc } & \text { Lambda } & R & \text { xbar } & \text { Area } \\ 0.01 & 0.00005 & 2.44486 & 1.00000 & 0.44992 & 0.55000 & 0.63596 \\ 1.00 & 0.04371 & 2.53956 & 0.93281 & 0.43443 & 0.55163 & 0.59292 \\ 2.00 & 0.05820 & 2.71710 & 0.82603 & 0.40848 & 0.55494 & 0.52419 \\ 3.00 & 0.05893 & 2.87183 & 0.74958 & 0.38879 & 0.55827 & 0.47488 \\ 5.00 & 0.05556 & 3.05399 & 0.67711 & 0.36882 & 0.56319 & 0.42735 \\ 10.00 & 0.05225 & 3.17605 & 0.64058 & 0.35745 & 0.56764 & 0.40140 \\ 20.00 & 0.05147 & 3.20145 & 0.63560 & 0.35540 & 0.56890 & 0.39681 \\ 50.00 & 0.05133 & 3.20557 & 0.63514 & 0.35510 & 0.56915 & 0.39615\end{array}$




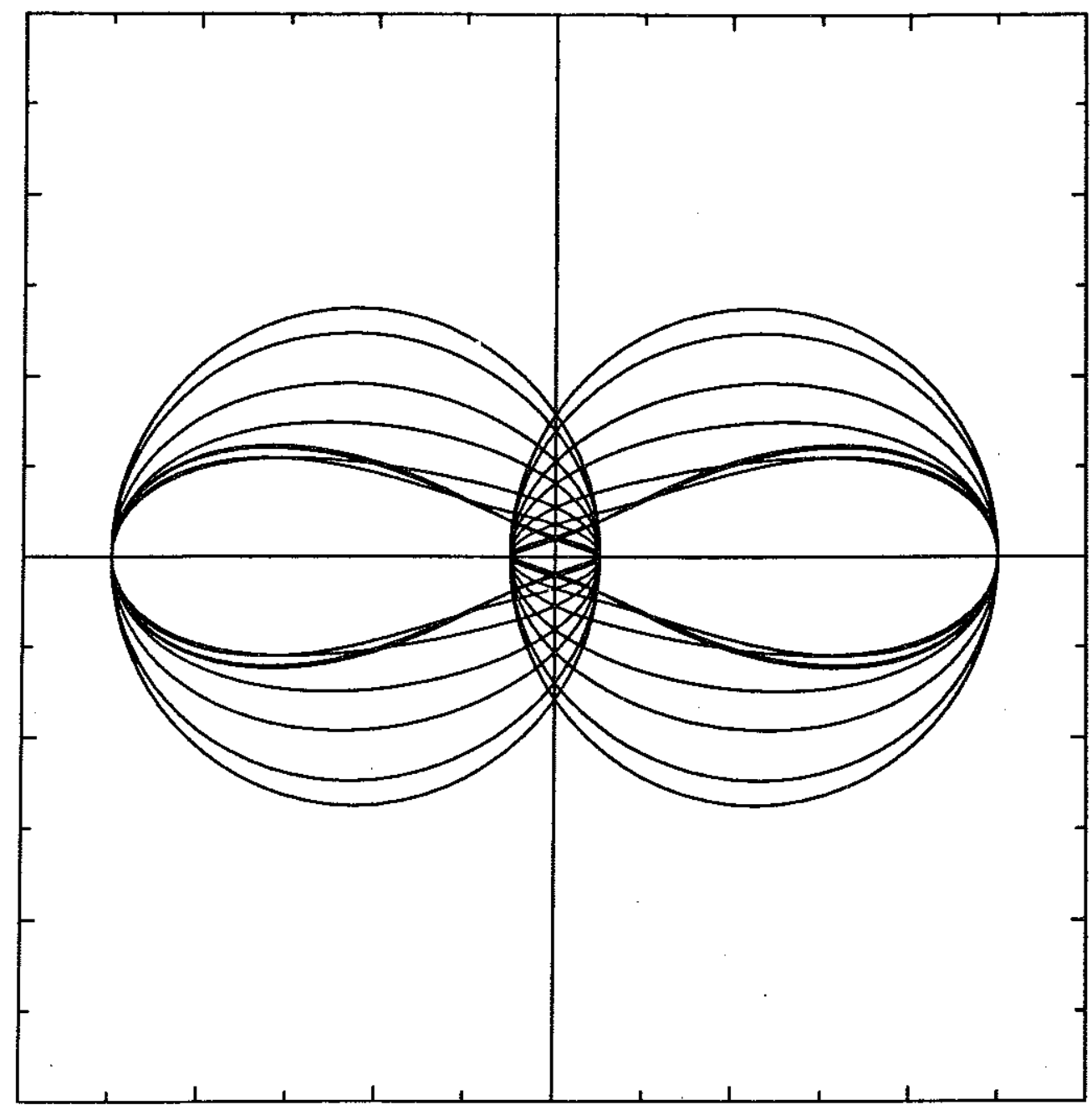

FIGURE 3.11. Doubly-connected two-layer corotating $V$-states for $v=-0.1$. The values of $\gamma$ are given below. As $\gamma$ increases the states become more elliptical.

Table $3.2 b$

$$
n u=-0.1
$$

$\begin{array}{rc}\text { Gamma } & \text { Omega } \\ 0.01 & 0.00007 \\ 1.00 & 0.07230 \\ 2.00 & 0.09336 \\ 3.00 & 0.08760 \\ 5.00 & 0.06930 \\ 8.00 & 0.05659 \\ 11.00 & 0.05364 \\ 11.50 & 0.05358 \\ 11.80 & 0.05359 \\ 11.85 & 0.05360 \\ 11.90 & 0.05360\end{array}$

dc

Lambda

$\mathbf{R}$

1.63664

1.73572

1. 98927

2.27876

2.78672

3.17126

3.24048

3.23624

3. 23226

3.23170

3.23127
1.00000

0.90004

0.69832

0.54012

0.38630

0.37273

0.43399

0.44804

0.45646

0.45767

0.45874
0.54991

0.52160

0.45972

0.40565

0.34528

0.32828

0.33481

0.33660

0.33765

0.33779

0.33791 xbar

0.45000

0.45268

0.45726

0.46219

0.48110

0.52054

0.54248

0.54466

0.54568

0.54582

0.54595
Area

0.95001

0.85473

0.66396

0.51695

0.37453

0.33857

0.35217

0.35594

0.35816

0.35846

0.35873 


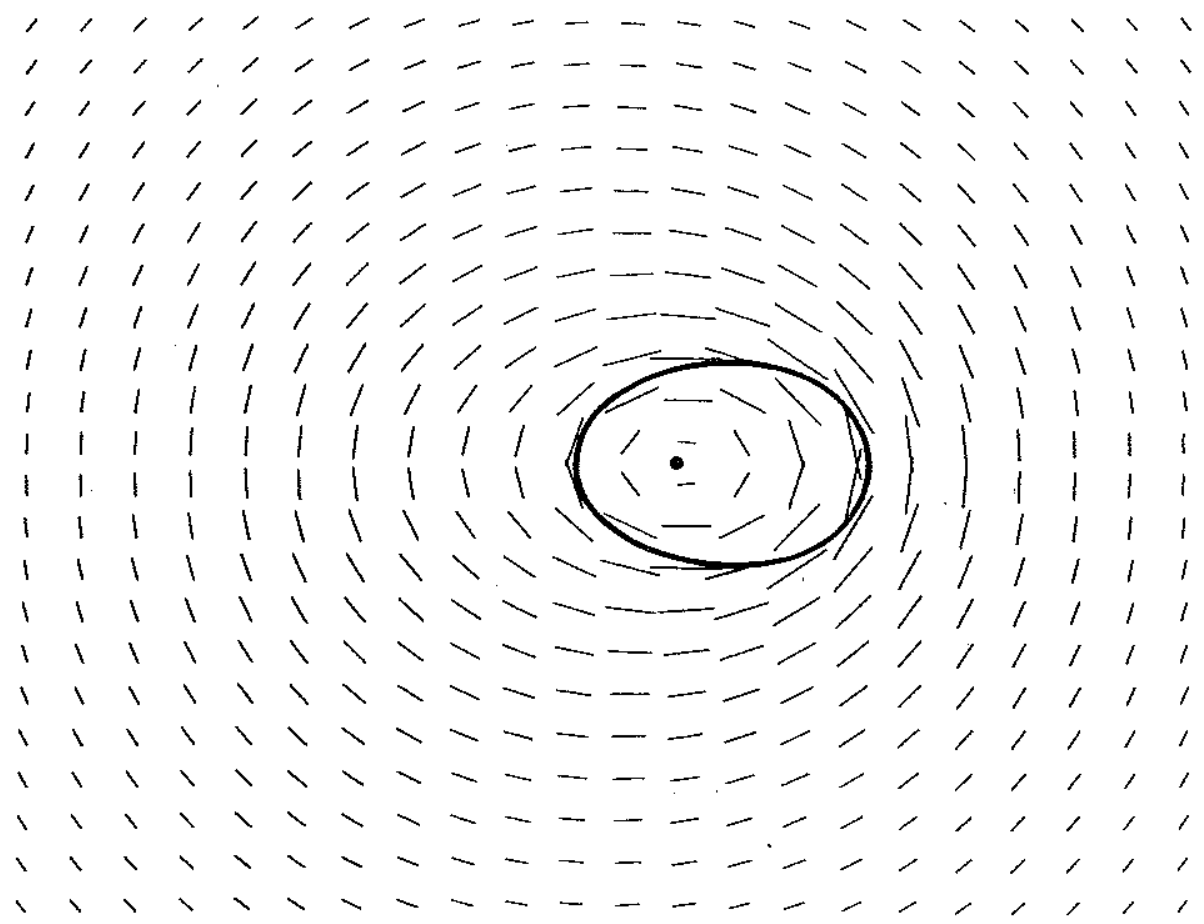

FIGURE 3.12a. The velocity field of the $V$-state $v=-0.1$ at $\gamma=2.0$ in the inertial frame. Note the presence of a single center, located somewhere inside the vortex. Note also that the velocity field is not tangent to the contour in this frame. 


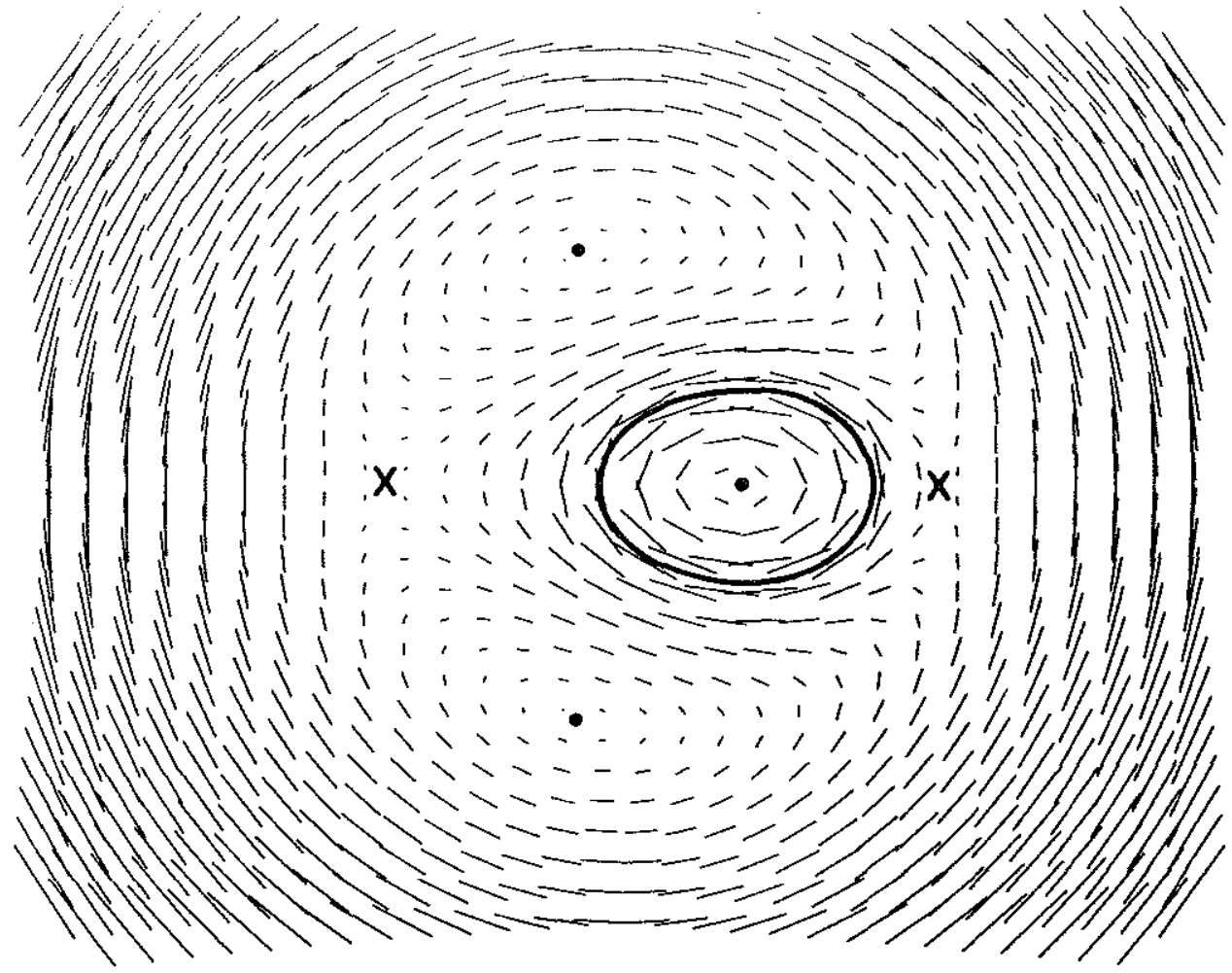

FIGURE 3.12b. The velocity field of the $V$-state $v=-0.1$ at $\gamma=2.0$ in the corotating frame. Note the presence of 3 centers (dots) and 2 saddles (crosses). 


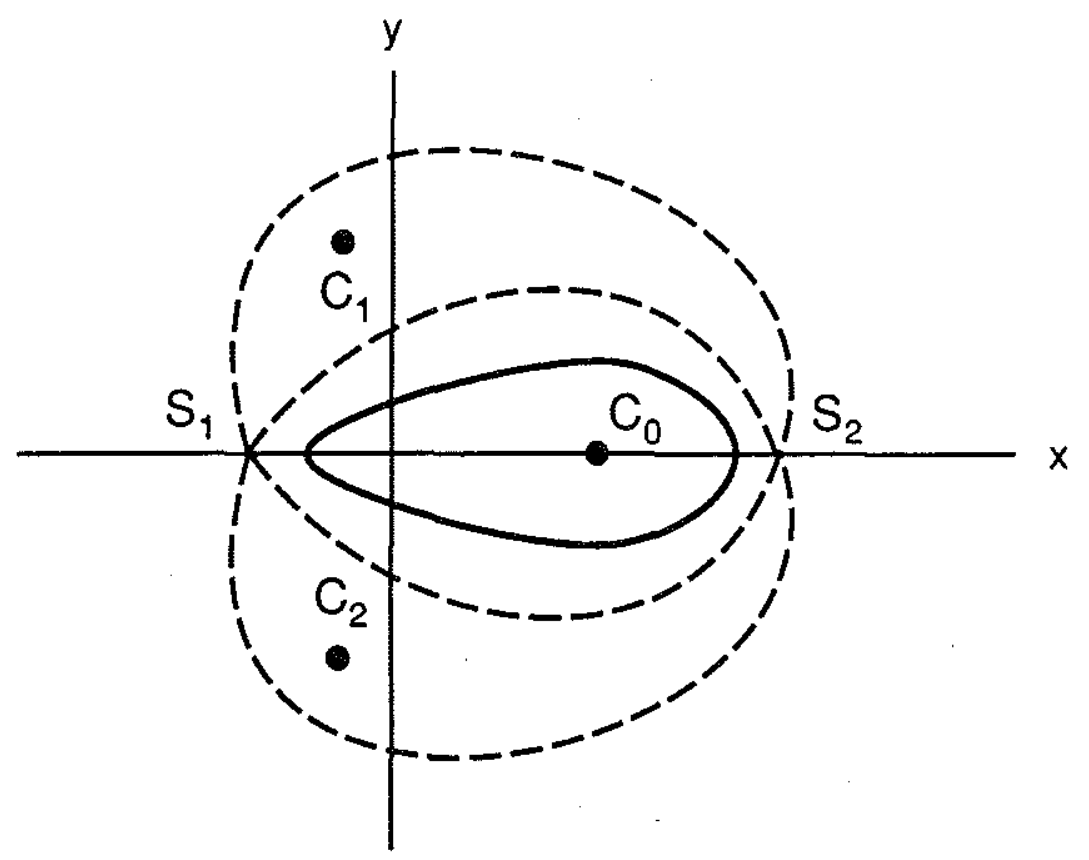

FIGURE 3.13. A sketch of the geometry of the corotating streamfunction in the upper layer for a doubly-connected corotating $V$-state, such as the one of the previous figure. In the lower layer the situation is identical but reflected about the $y$-axis. 


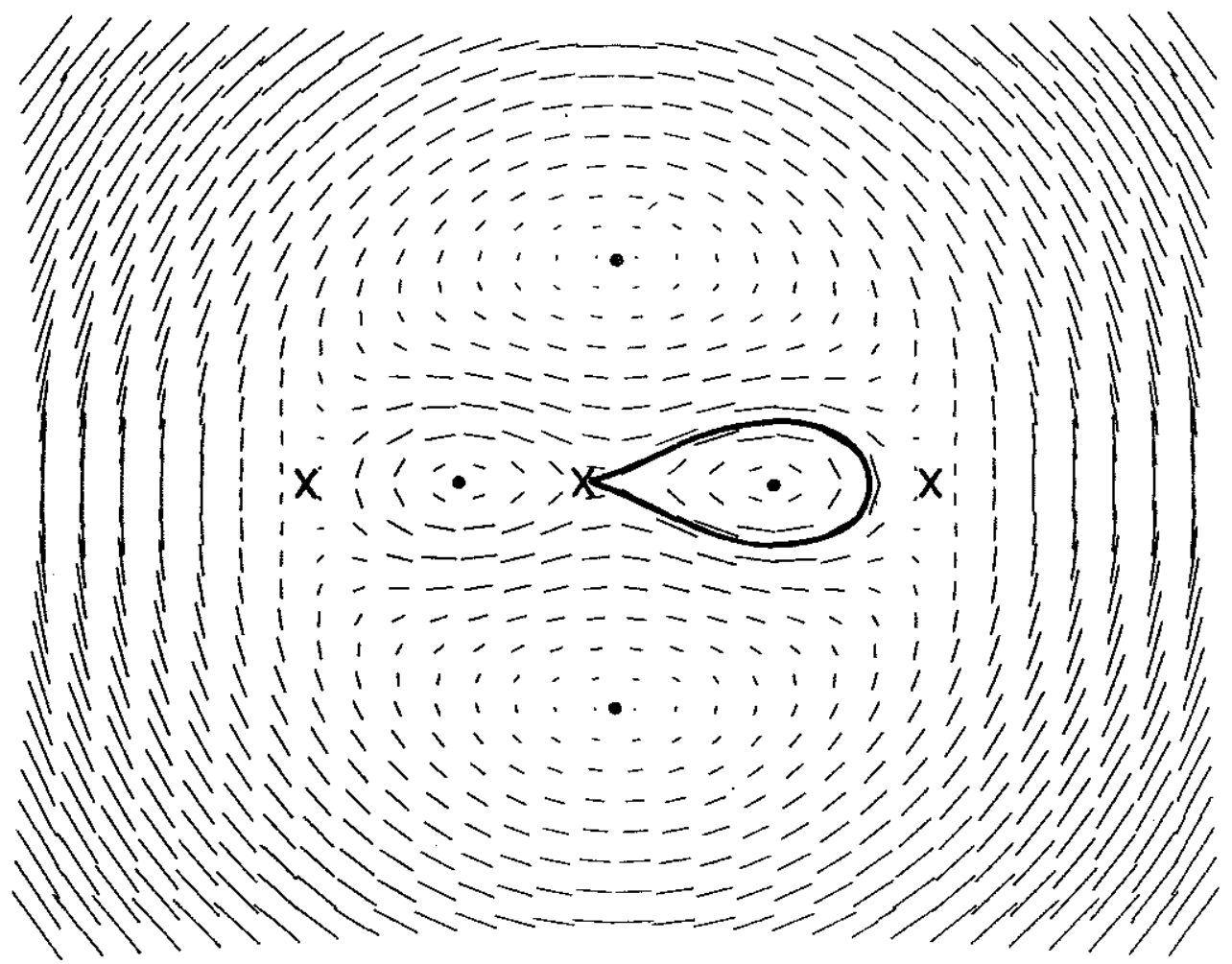

FIGURE 3.14. The velocity field of the V-state $v=-0.1$ at $\gamma=\gamma_{c} \approx 11.90$ in the corotating frame. Note the appearance of a new center and a new saddle on the $x$-axis. 


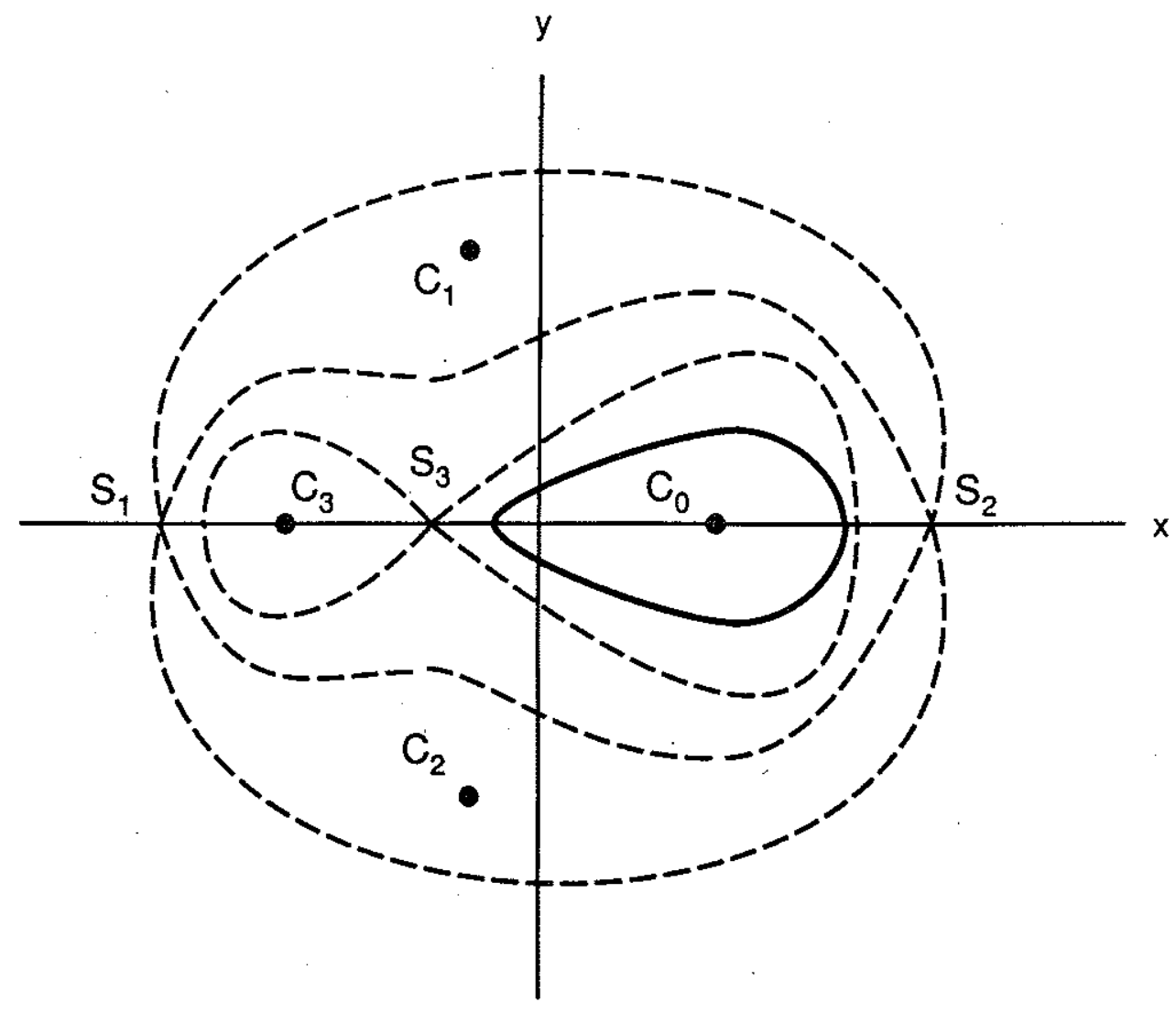

FIGURE 3.15. A sketch of the geometry of the corotating streamfunction in the upper layer for a doubly-connected corotating $V$-state, such as the one of the previous figure. 


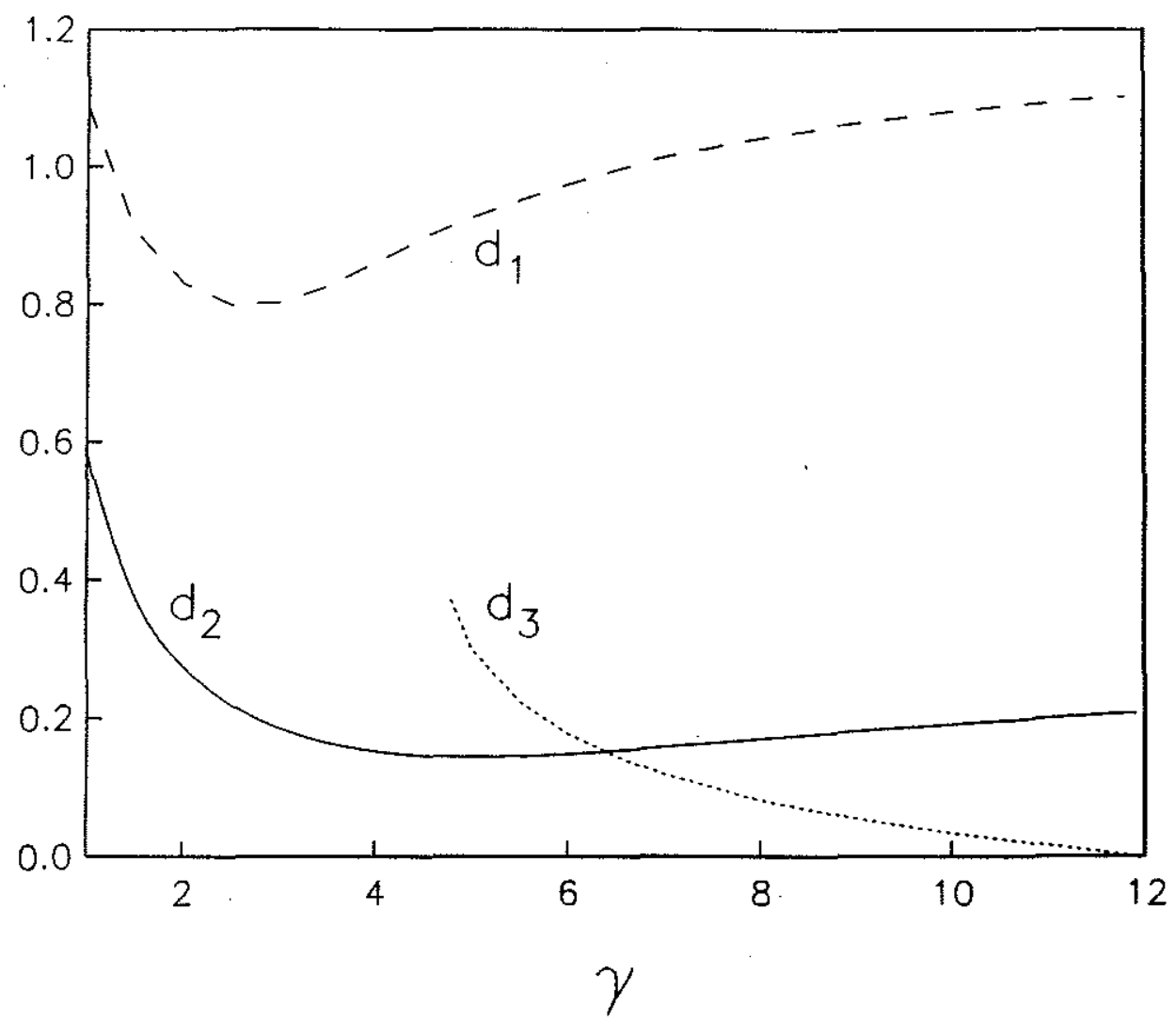

FIGURE 3.16. Plots of the distances between the critical points and the contour as a function of $\gamma$ for the doubly-connected rotating $V$-states with $v=-0.1$. 


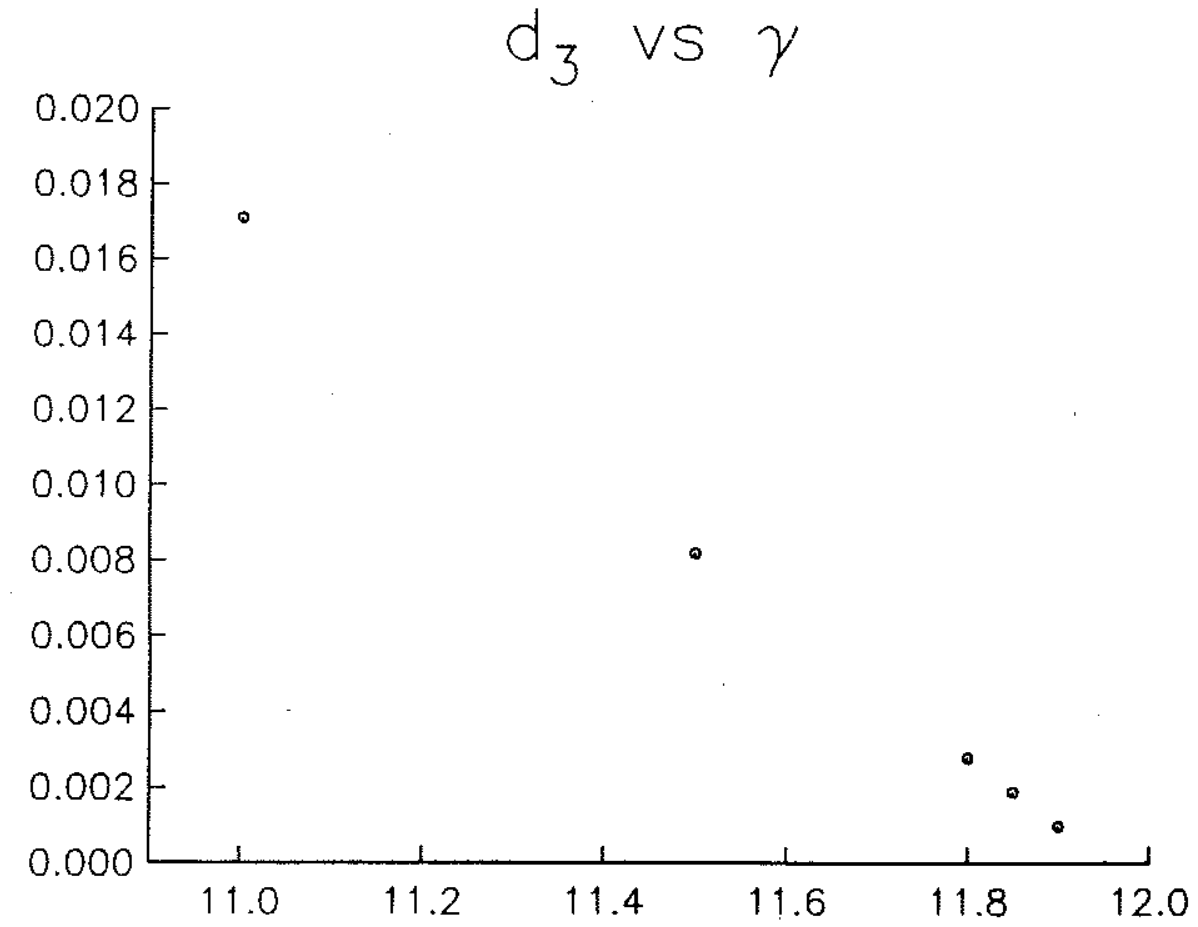

FIGURE 3.16a An enlargement of the previous figure near $\gamma_{c}$ showing the intersection of $d_{3}$ with the axis at $\gamma=11.90$. 


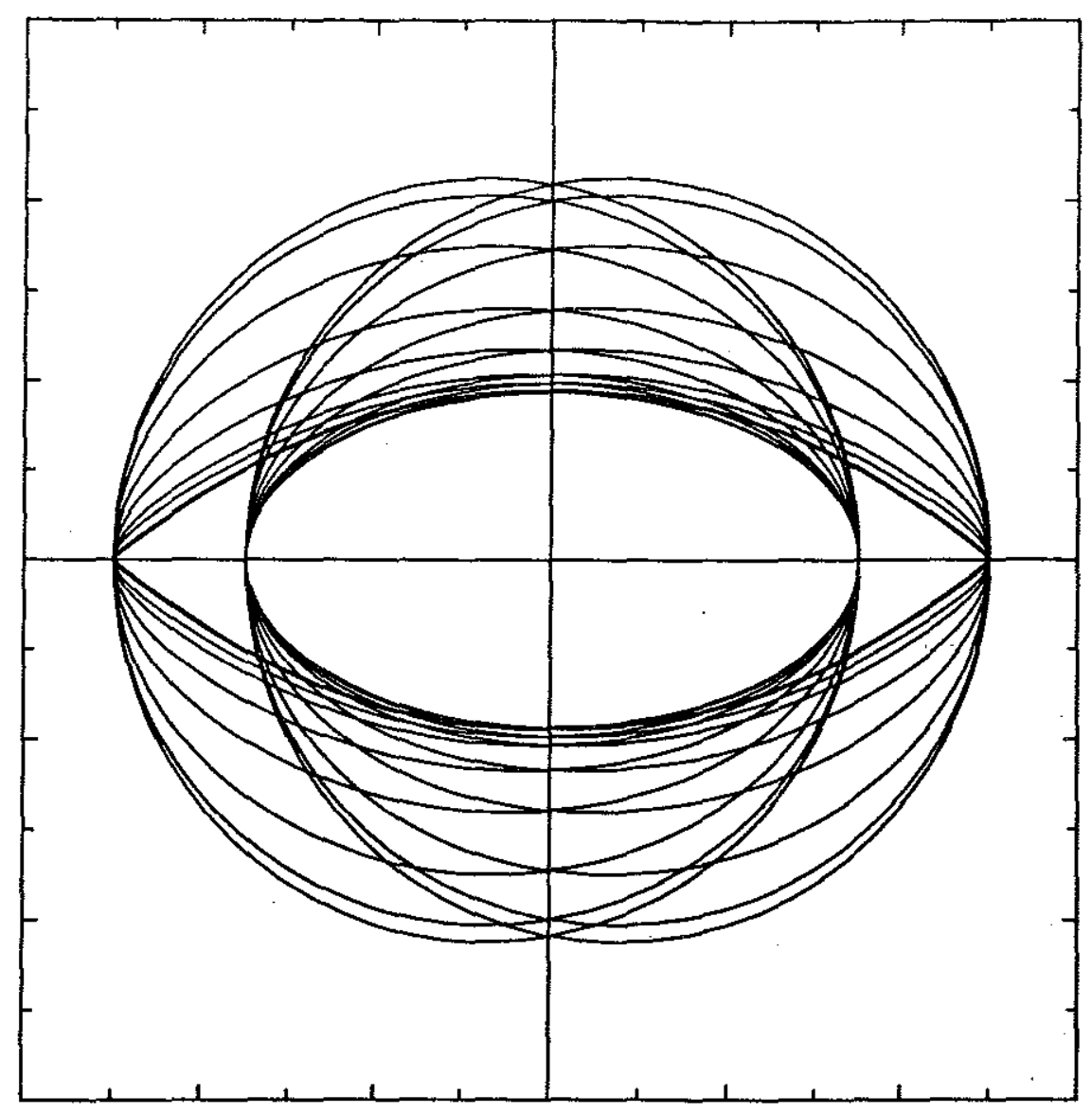

FIGURE 3.17. Doubly-connected two-layer corotating $V$-states for $v=-0.7$. The values of $\gamma$ are given in the table below. As $\gamma$ increases the states become more elliptical.

$$
\begin{gathered}
\text { Table } 3.2 \mathrm{c} \\
\mathrm{nu}=-0.7
\end{gathered}
$$

$\begin{array}{lcccccc}\text { Gamma } & \text { Omega } & \text { dc } & \text { Lambda } & \text { R } & \text { xbar } & \text { Area } \\ 0.01 & 0.00017 & 0.35300 & 1.00000 & 0.84986 & 0.15000 & 2.26904 \\ 1.00 & 0.17724 & 0.36006 & 0.95523 & 0.83076 & 0.14956 & 2.16820 \\ 1.50 & 0.21774 & 0.37663 & 0.82323 & 0.77082 & 0.14516 & 1.86661 \\ 2.00 & 0.22059 & 0.39119 & 0.65986 & 0.68809 & 0.13459 & 1.48744 \\ 2.50 & 0.21458 & 0.38658 & 0.55120 & 0.62452 & 0.12071 & 1.22530 \\ 3.00 & 0.20891 & 0.35894 & 0.48710 & 0.57933 & 0.10397 & 1.05439 \\ 3.25 & 0.20722 & 0.33486 & 0.46749 & 0.56160 & 0.09403 & 0.99084 \\ 3.50 & 0.20666 & 0.30149 & 0.45444 & 0.54517 & 0.08218 & 0.93371 \\ 3.55 & 0.20663 & 0.29419 & 0.45208 & 0.54168 & 0.07968 & 0.92180\end{array}$




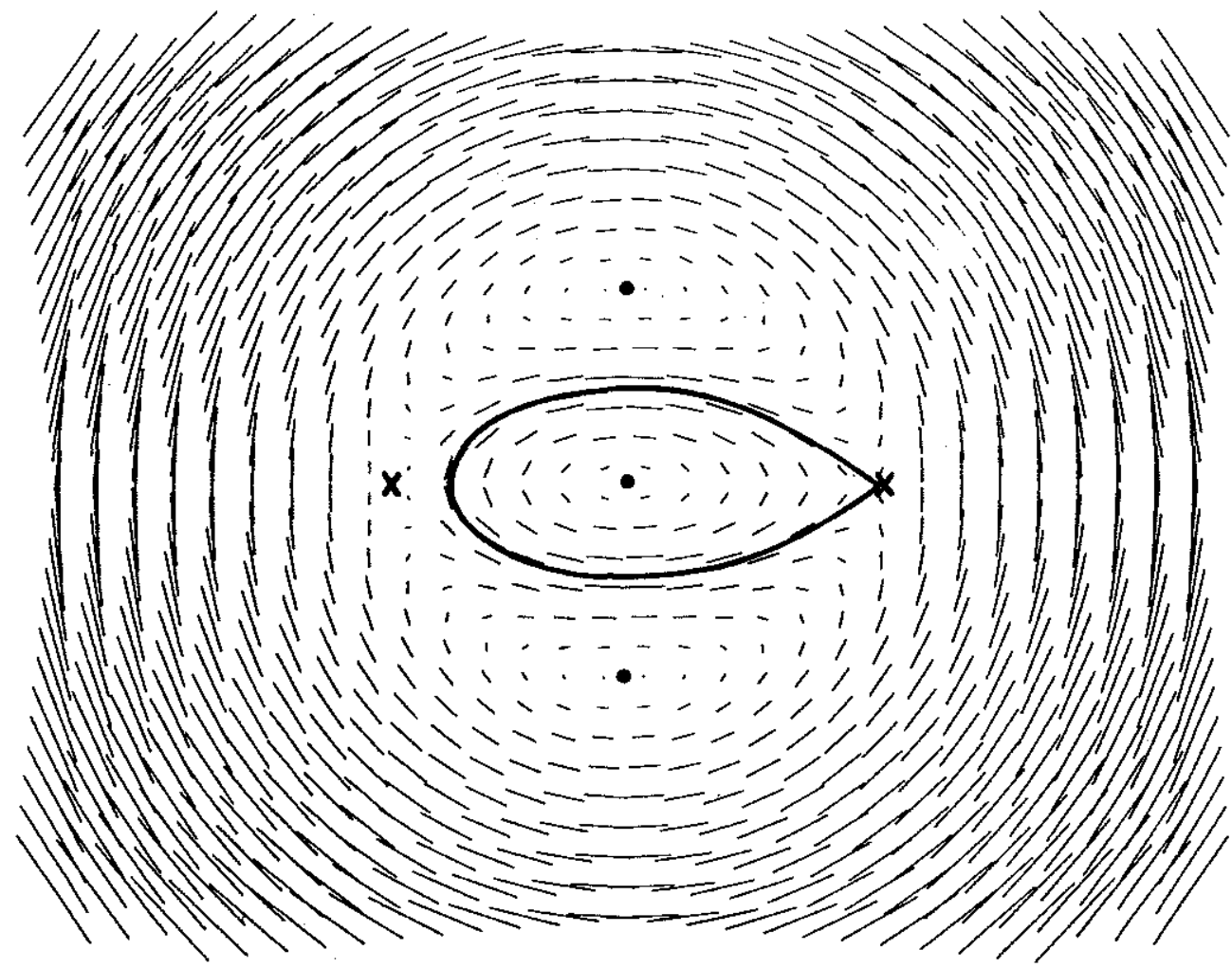

FIGURE 3.18. The velocity field of the V-state $v=-0.7$ at $\gamma=\gamma_{c}=3.55$ in the corotating frame. Note the presence of 3 centers (dots) and 2 saddles (crosses). 


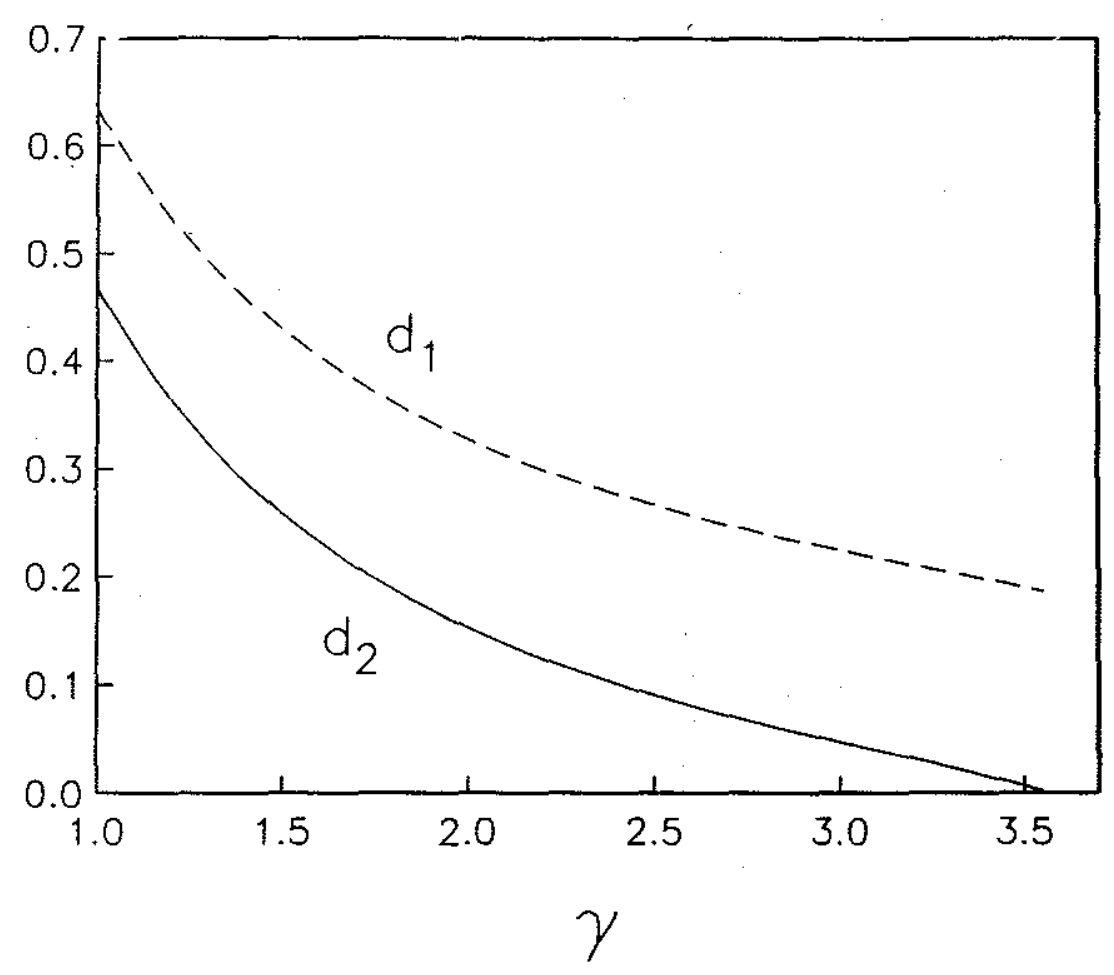

FIGURE 3.19. Plots of the distances between the critical points and the contour as a function of $\gamma$ for the doubly-connected rotating $V$-states with $v=-0.7$. 


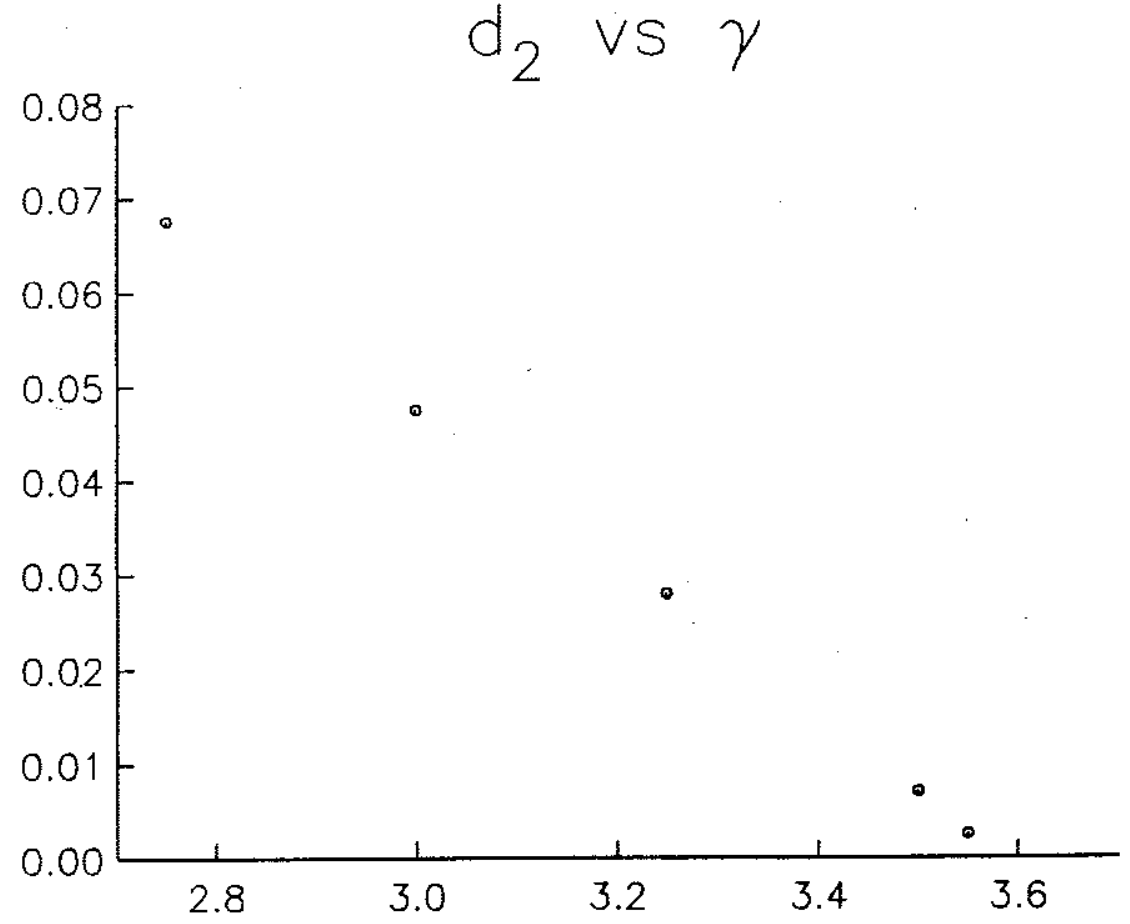

FIgURE 3.19a An enlargement of the previous figure near $\gamma_{c}$ showing the intersection of $d_{3}$ with the axis at $\gamma \approx 3.55$. 


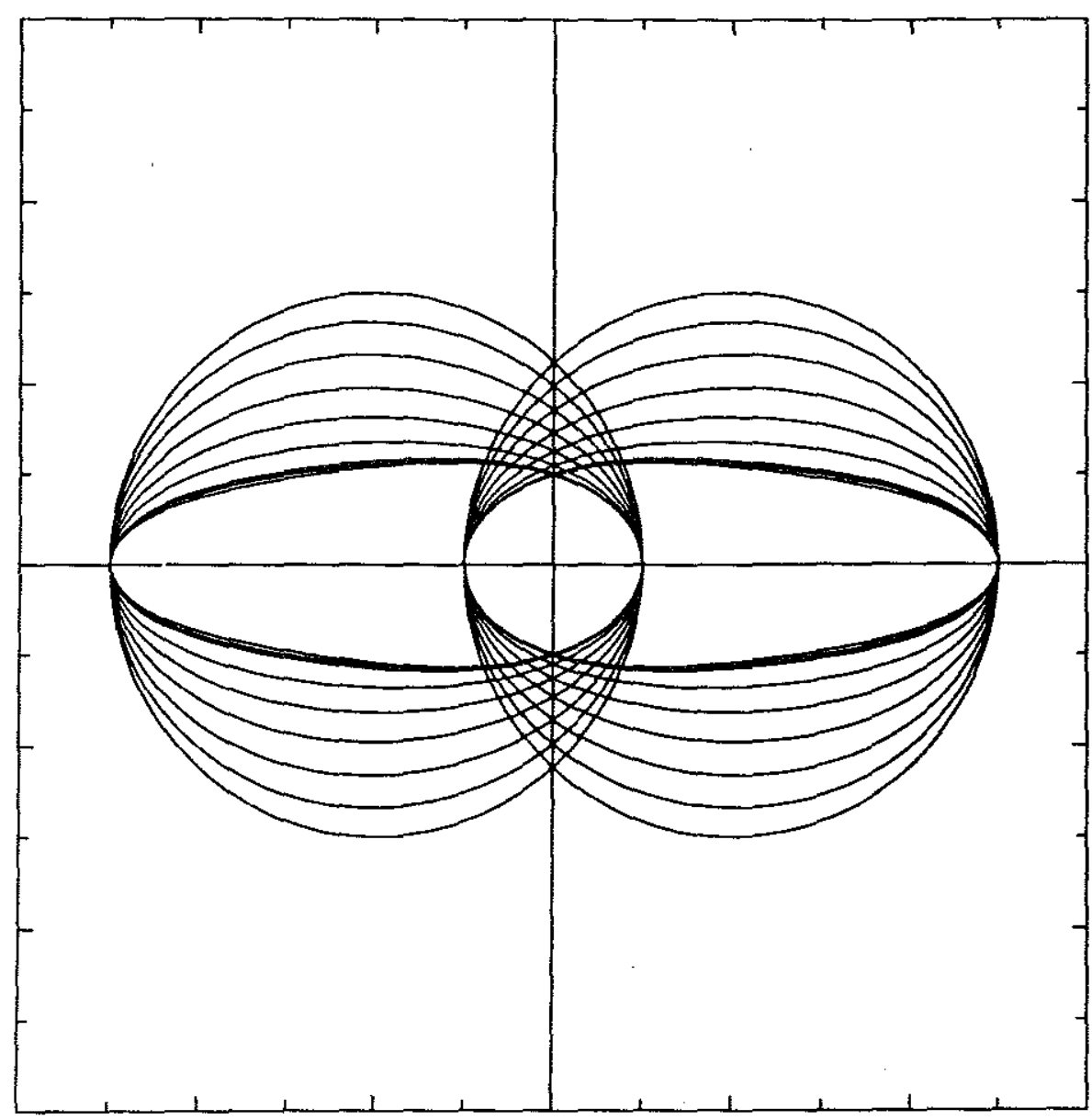

FIGURE 3.20. Doubly-connected two-layer corotating $V$-states for $v=-0.2$. The values of $\gamma$ are given in the table below. As $\gamma$ increases the states become more elliptical.

Table $3.2 \mathrm{~d}$

nu $=-0.2$

$\begin{array}{lcccccc}\text { Gamma } & \text { Omega } & \text { dc } & \text { Lambda } & R & \text { xbar } & \text { Area } \\ 0.01 & 0.00009 & 1.33356 & 1.00000 & 0.59990 & 0.40000 & 1.13059 \\ 1.00 & 0.08902 & 1.41882 & 0.89272 & 0.56695 & 0.40220 & 1.00980 \\ 1.50 & 0.10945 & 1.52563 & 0.77404 & 0.52832 & 0.40301 & 0.87690 \\ 2.00 & 0.11408 & 1.65499 & 0.65133 & 0.48560 & 0.40183 & 0.74082 \\ 2.50 & 0.11115 & 1.78839 & 0.54393 & 0.44530 & 0.39819 & 0.62296 \\ 3.00 & 0.10530 & 1.91373 & 0.45296 & 0.40832 & 0.39071 & 0.52379 \\ 3.40 & 0.10012 & 1.99216 & 0.38540 & 0.37780 & 0.37632 & 0.44842 \\ 3.45 & 0.09954 & 1.99674 & 0.37621 & 0.37314 & 0.37254 & 0.43742 \\ 3.50 & 0.09905 & 1.99648 & 0.36613 & 0.36762 & 0.36697 & 0.42456\end{array}$




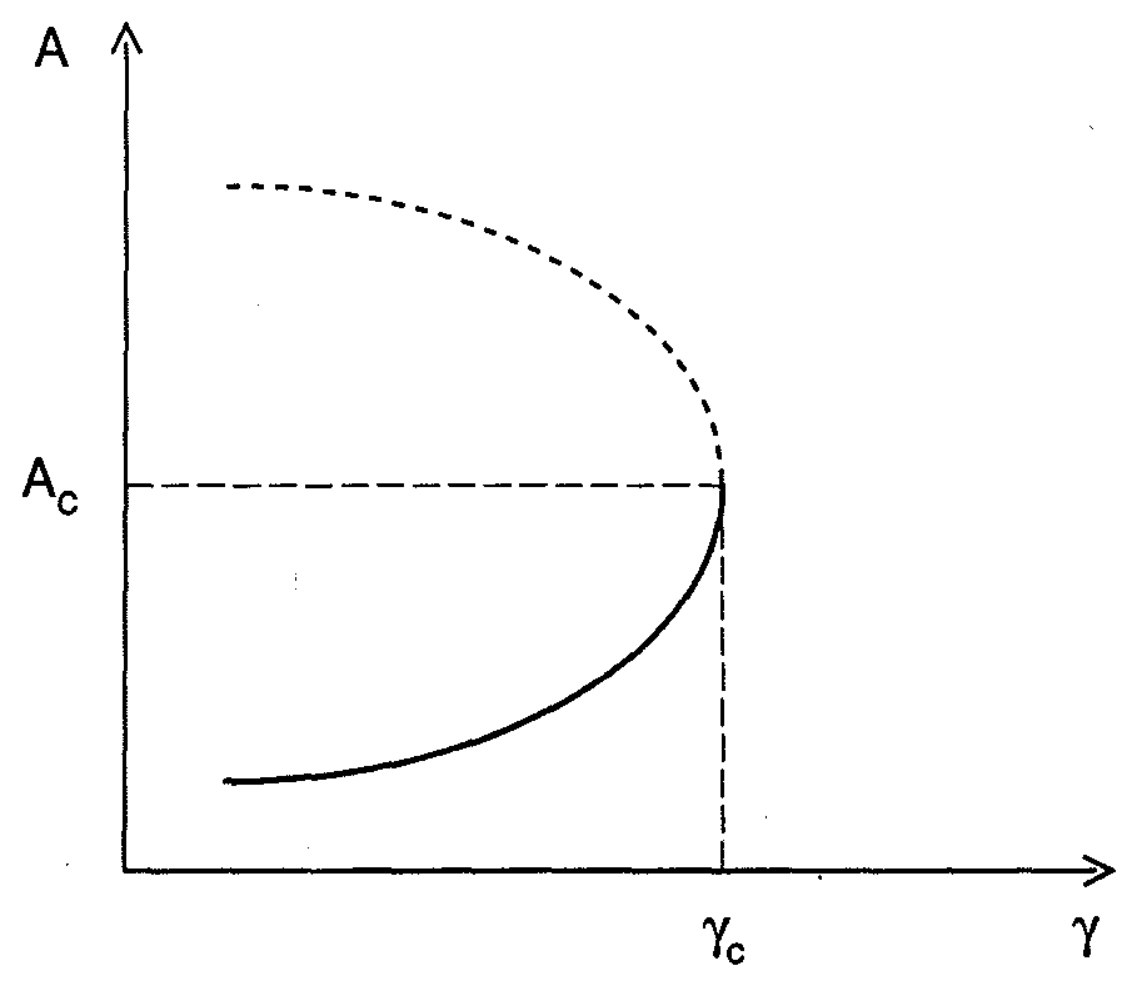

FIGURE 3.21. A schematic drawing of the solution in the presence of a fold. 


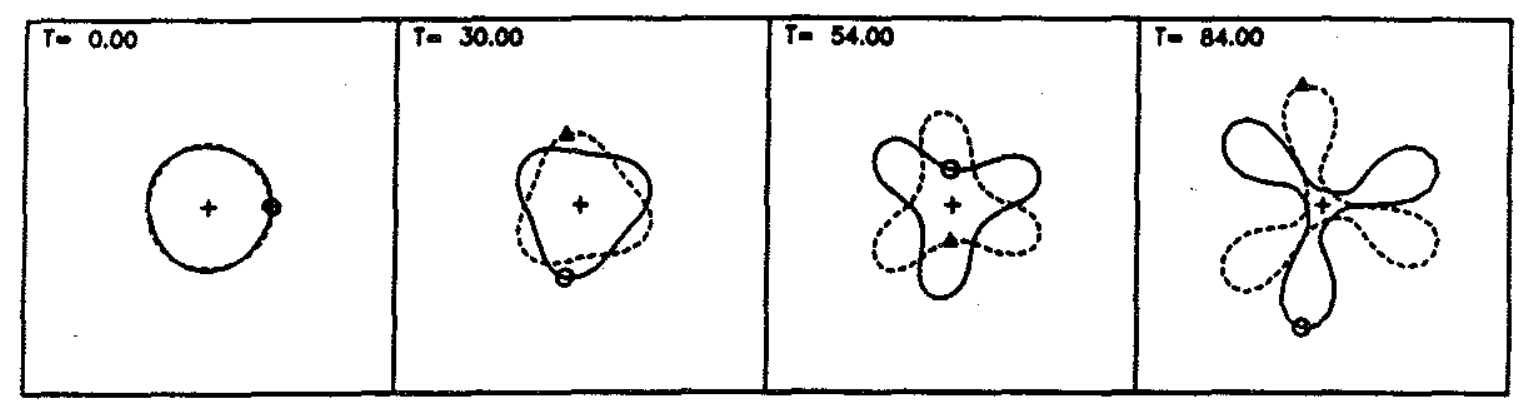

FIGURE 3.22. The evolution of an unstable circular baroclinic vortex with a $m=3$ normal mode perturbation (reprinted from Helfrich and Send, 1988). 


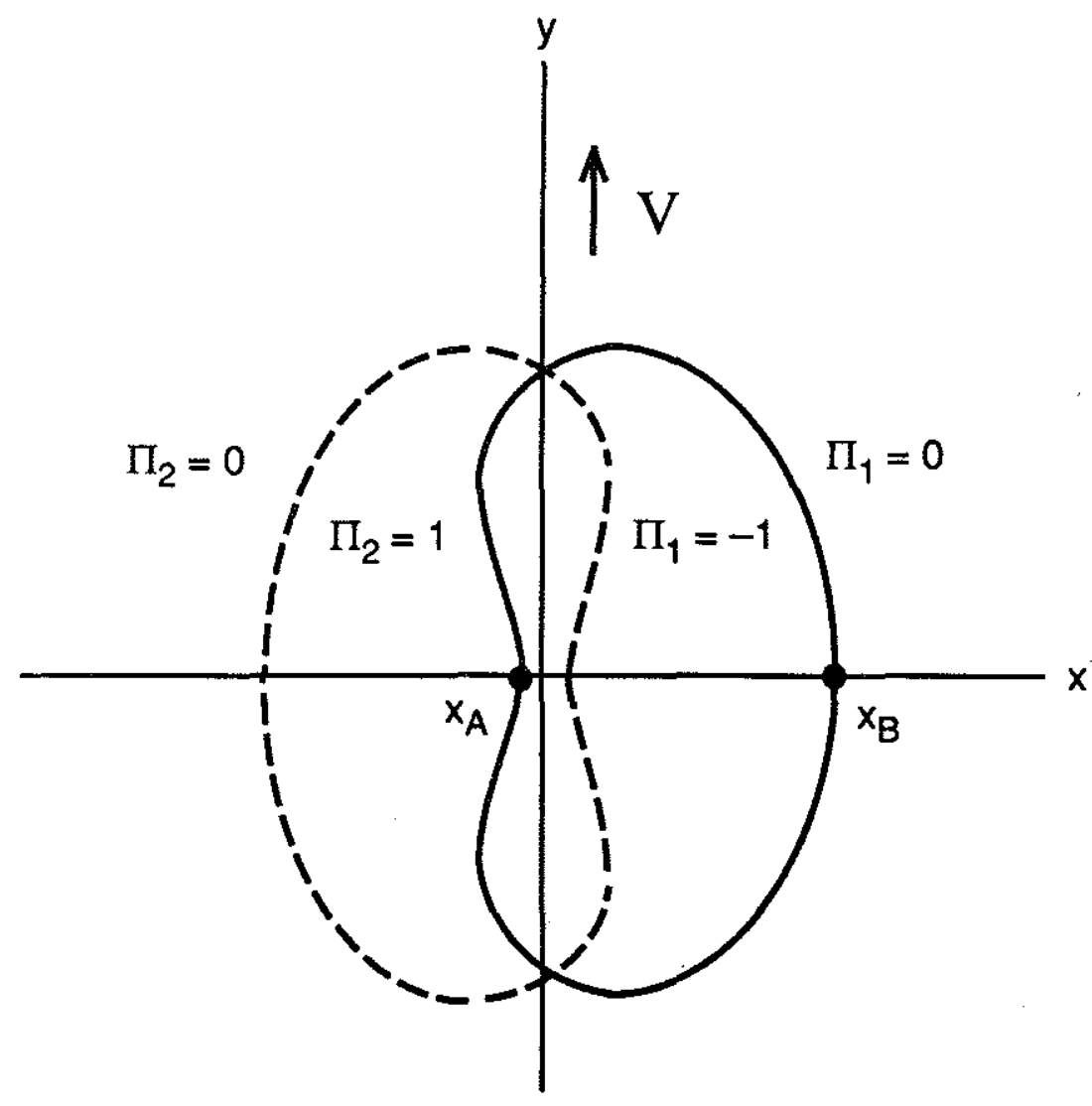

FIGURE 3.23. A schematic drawing of a two-layer dipole. The solid (dashed) contour is in the upper (lower) layer. Note that the two vortices can overlap $\left(\mu=x_{A} / x_{B}\right)$. 


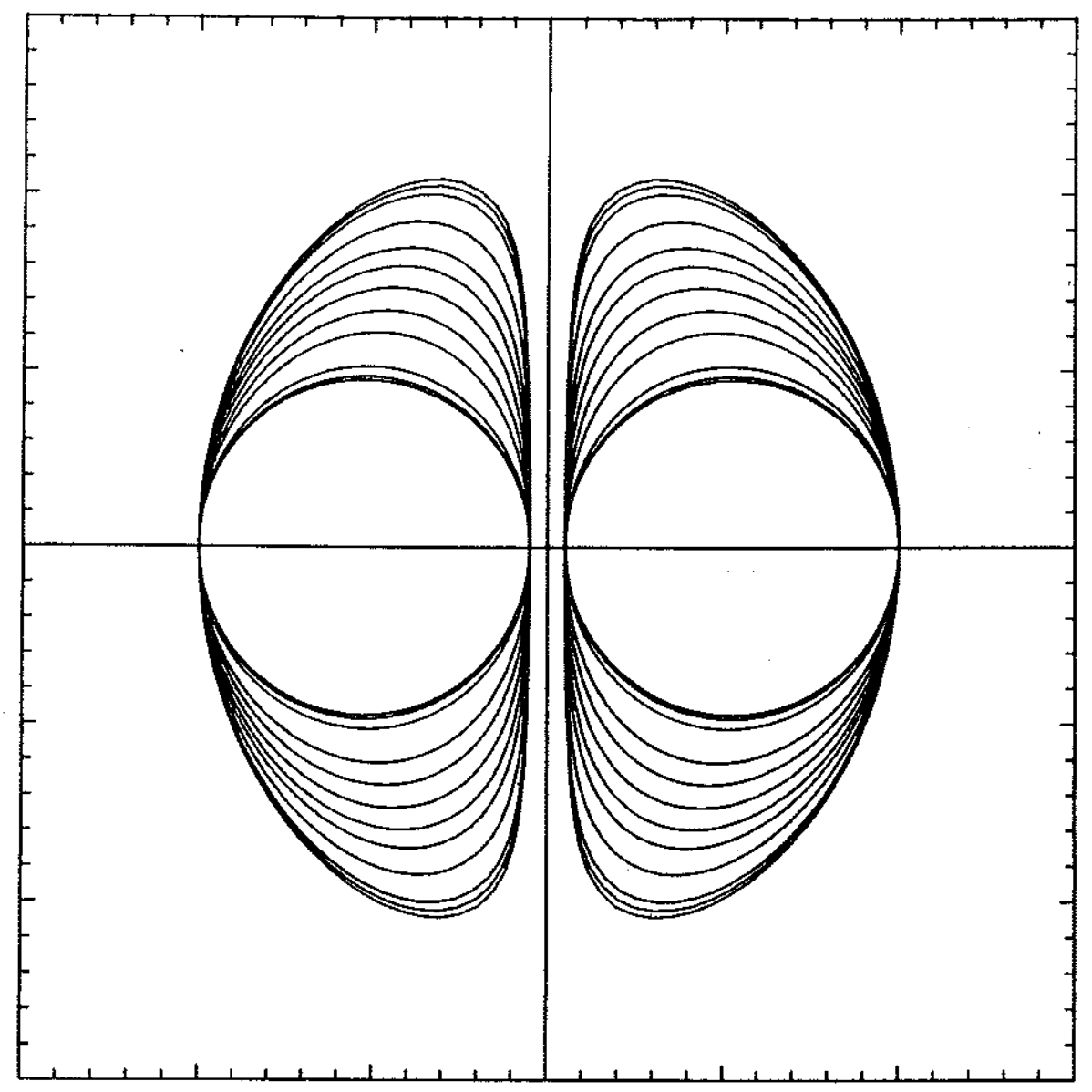

FIGURE 3.24. Two-layer dipoles for $\mu=0.05$. The values of $\gamma$ are given in the table below. As $\gamma$ increases the vortices become more elongated.

Table $3.3 a$

$\mathrm{mu}=0.05$

$\begin{array}{rccccccc}\text { Gamma } & \mathrm{V} & \mathrm{V} / \mathrm{Vpv} & \text { Area } & \text { Area/AO } & \text { dc } & \text { Lambda } & R \\ 0.010 & 0.00003 & 0.97855 & 0.70883 & 1.00001 & 1.05000 & 0.99929 & 0.47500 \\ 0.500 & 0.01446 & 0.92608 & 0.72475 & 1.02248 & 1.04882 & 0.97830 & 0.48031 \\ 1.000 & 0.03051 & 0.90447 & 0.76806 & 1.08357 & 1.04483 & 0.92410 & 0.49445 \\ 2.000 & 0.05406 & 0.87460 & 0.91814 & 1.29531 & 1.02762 & 0.77697 & 0.54061 \\ 2.500 & 0.06329 & 0.85562 & 1.01683 & 1.43454 & 1.01524 & 0.70415 & 0.56892 \\ 3.000 & 0.07125 & 0.83377 & 1.12074 & 1.58113 & 1.00205 & 0.64100 & 0.59728 \\ 3.500 & 0.07779 & 0.81232 & 1.21820 & 1.71863 & 0.98986 & 0.59093 & 0.62271 \\ 4.000 & 0.08285 & 0.79413 & 1.30058 & 1.83485 & 0.97988 & 0.55381 & 0.64342 \\ 5.000 & 0.08932 & 0.76980 & 1.41475 & 1.99592 & 0.96671 & 0.50832 & 0.67107 \\ 7.500 & 0.09535 & 0.74717 & 1.53044 & 2.15913 & 0.95419 & 0.46754 & 0.69796 \\ 10.000 & 0.09704 & 0.74073 & 1.56484 & 2.20767 & 0.95053 & 0.45624 & 0.70577 \\ 50.000 & 0.09825 & 0.73546 & 1.59085 & 2.24436 & 0.94765 & 0.44793 & 0.71161\end{array}$




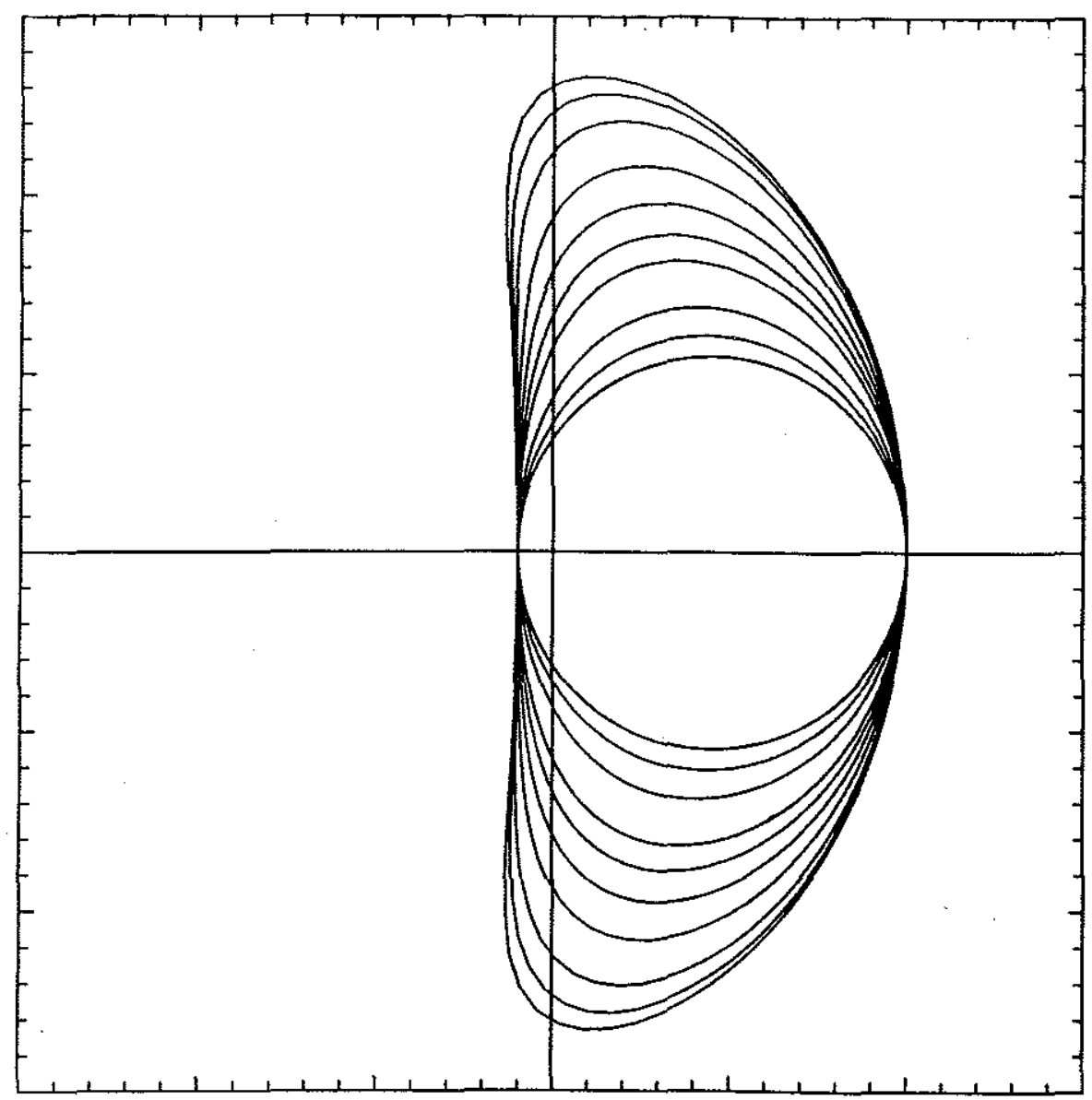

FIGURE 3.25. Two-layer dipoles for $\mu=-0.1$ (only the upper layer vortex is shown). The values of $\gamma$ are given in the table below. As $\gamma$ increases the vortices become more elongated.

Table $3.3 b$

mu $=-0.1$

$\begin{array}{cccccccc}\text { Gamma } & \mathrm{V} & \mathrm{V} / \mathrm{Vpv} & \text { Area } & \text { Area/AO } & \text { dc } & \text { Lambda } & \mathrm{R} \\ 0.010 & 0.00003 & 0.96494 & 0.95034 & 1.00000 & 0.90000 & 0.99929 & 0.55000 \\ 0.100 & 0.00170 & 0.93152 & 0.95133 & 1.00105 & 0.89994 & 0.99875 & 0.55029 \\ 1.000 & 0.03896 & 0.82575 & 1.05424 & 1.10934 & 0.89189 & 0.90266 & 0.57929 \\ 1.500 & 0.05830 & 0.78098 & 1.19816 & 1.26078 & 0.87783 & 0.79650 & 0.61757 \\ 2.000 & 0.07730 & 0.71907 & 1.43759 & 1.51272 & 0.85219 & 0.66760 & 0.67646 \\ 2.200 & 0.08513 & 0.68591 & 1.57164 & 1.65378 & 0.83743 & 0.61251 & 0.70730 \\ 2.400 & 0.09309 & 0.64691 & 1.73387 & 1.82448 & 0.81940 & 0.55697 & 0.74290 \\ 2.600 & 0.10101 & 0.60167 & 1.92856 & 2.02936 & 0.79729 & 0.50210 & 0.78351 \\ 2.800 & 0.10859 & 0.54856 & 2.16298 & 2.27602 & 0.76860 & 0.44818 & 0.82976 \\ 2.900 & 0.11205 & 0.51546 & 2.30516 & 2.42564 & 0.74801 & 0.42013 & 0.85660 \\ 2.950 & 0.11351 & 0.49237 & 2.39501 & 2.52018 & 0.73144 & 0.40364 & 0.87313\end{array}$




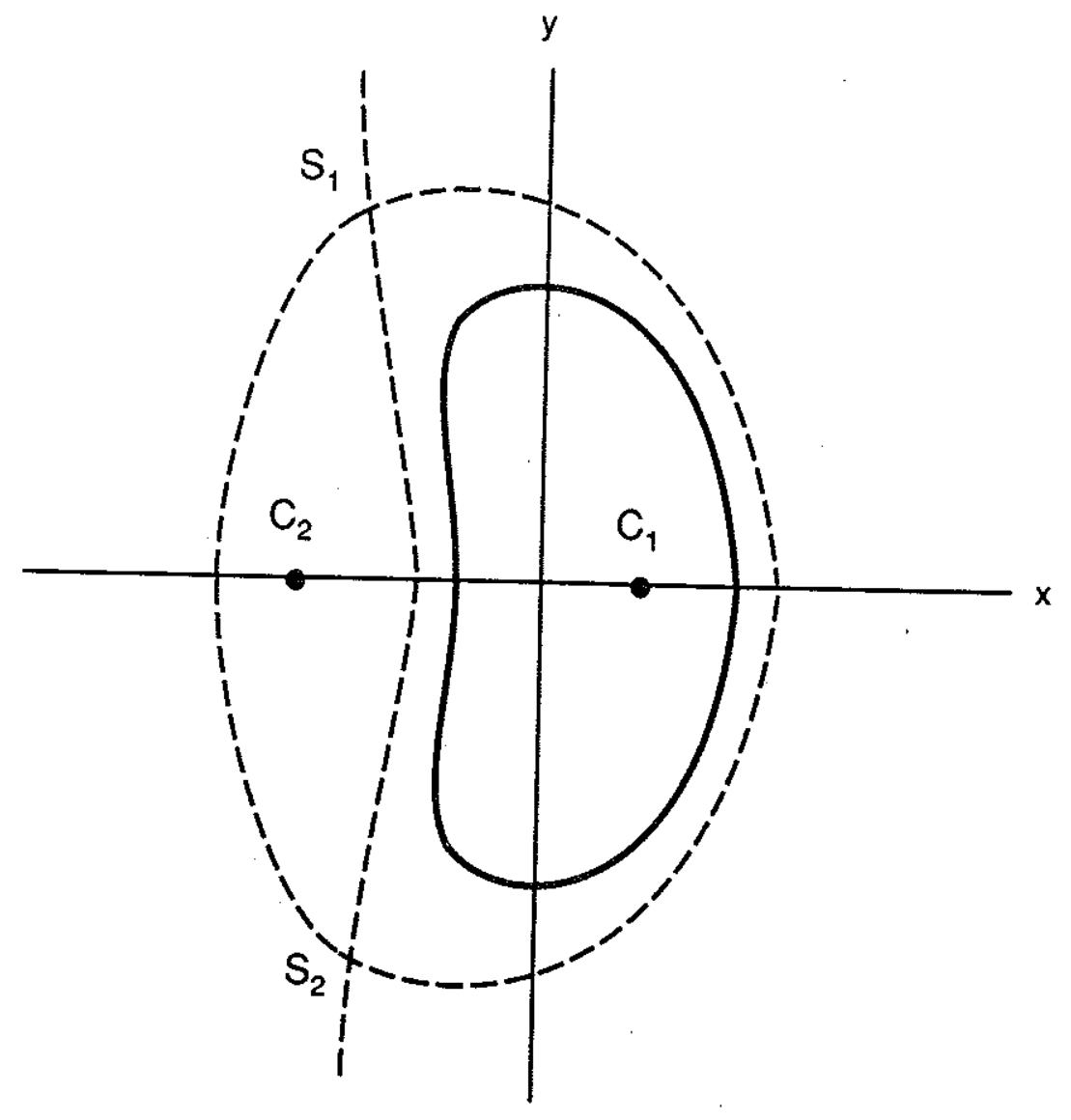

FIGURE 3.26. A sketch of the corotranslating streamfunction for the two-layer dipoles when $v$ is not too negative and $\gamma$ not too large. 


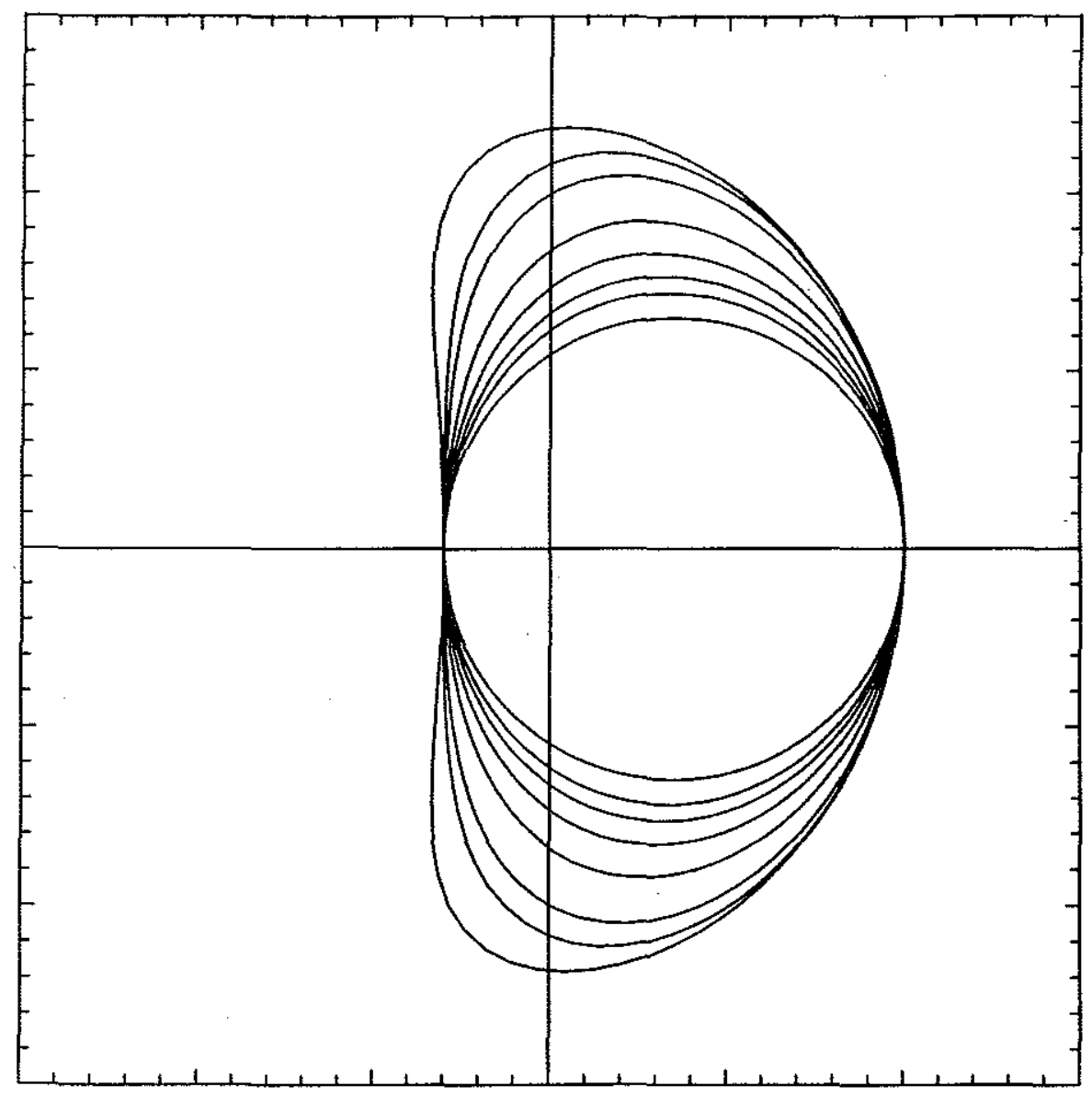

FIGURE 3.27. Two-layer dipoles for $\mu=-0.3$ (only tho upper layer vortex is shown). The values of $\gamma$ are given in the table below. As $\gamma$ increases the vortices become more elongated.

\begin{tabular}{|c|c|c|c|c|c|c|c|}
\hline \multicolumn{8}{|c|}{$m u=-0.3$} \\
\hline Gamma & V & $\mathrm{V} / \mathrm{Vpv}$ & Area & Area/A0 & $d c$ & Iambda & $\mathrm{R}$ \\
\hline 0.010 & 0.00004 & 0.92540 & 1.32733 & 1.00000 & 0.70000 & 0.99898 & 0.65000 \\
\hline 1.000 & 0.04405 & 0.66961 & 1.47023 & 1.10767 & 0.69236 & 0.90298 & 0.68410 \\
\hline 1.250 & 0.05535 & 0.62953 & 1.56799 & 1. 18131 & 0.68533 & 0.84731 & 0.70647 \\
\hline 1.500 & 0.06627 & 0.58633 & 1.70671 & 1.28583 & 0.67380 & 0.77990 & 0.73706 \\
\hline 1.750 & 0.07694 & 0.53519 & 1.90452 & 1.43486 & 0.65471 & 0.70187 & 0.77861 \\
\hline 2.000 & 0.08684 & 0.46762 & 2.19558 & 1.65414 & 0.61966 & 0.61464 & 0.83599 \\
\hline 2.100 & 0.08979 & 0.43014 & 2.35450 & 1.77387 & 0.59381 & 0.57721 & 0.86571 \\
\hline 2.200 & 0.08805 & 0.36363 & 2.56200 & 1.93020 & 0.52997 & 0.53637 & 0.90306 \\
\hline
\end{tabular}




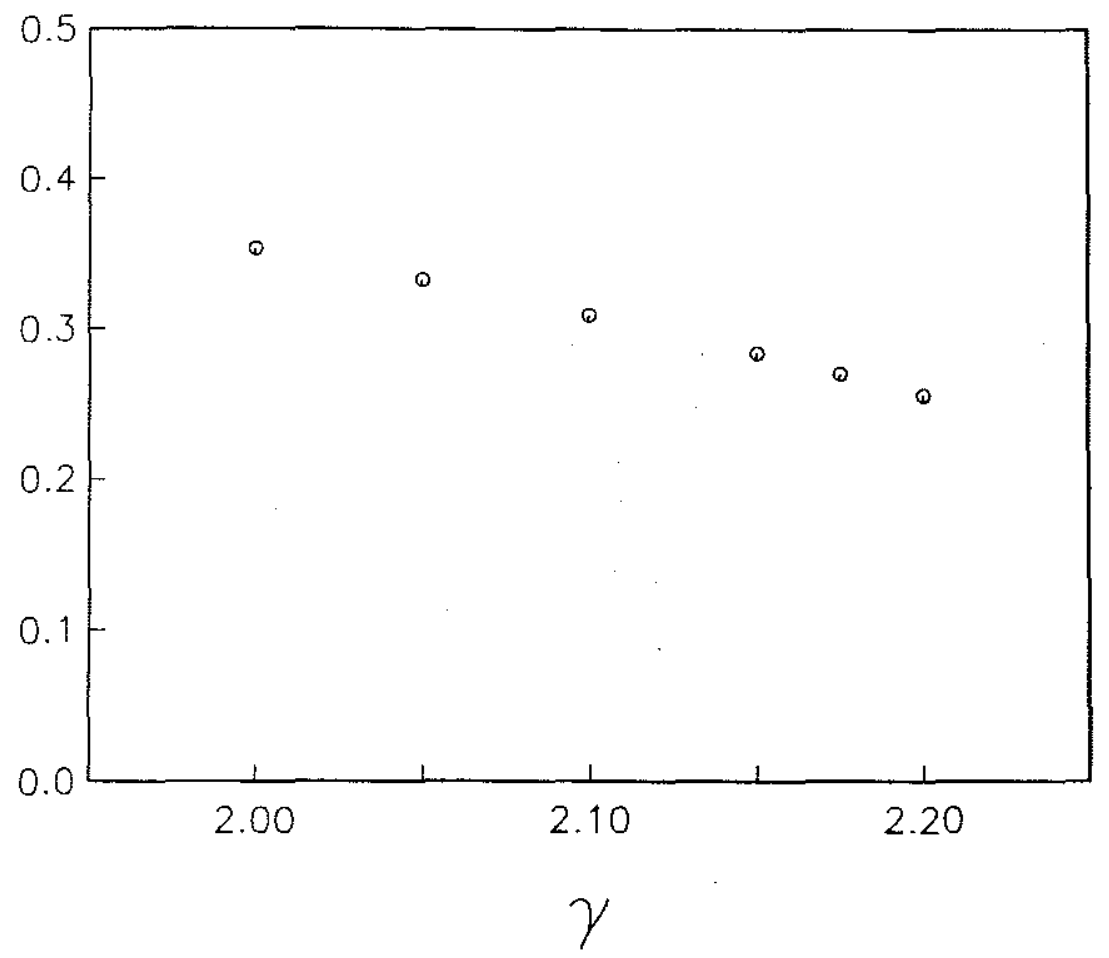

FIGURE 3.28. The distance between the critical point $S_{1}$ and the contour for the twolayer dipole with $\mu=-0.3$. 


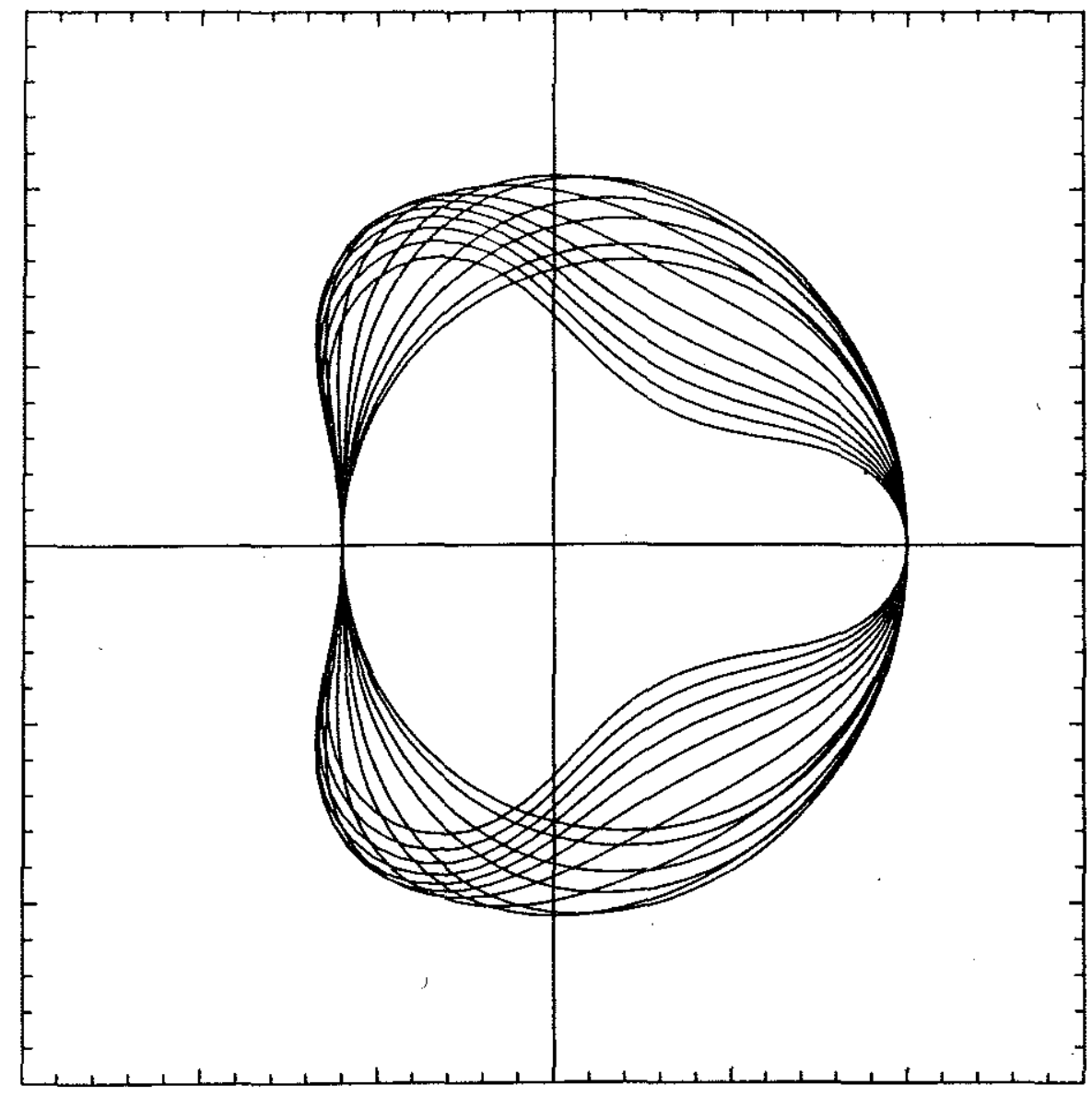

FIGURE 3.29. Two-layer dipoles for $\mu=-0.6$ (only the upper layer vortex is shown). The values of $\gamma$ are given in the table below. As $\gamma$ increases the vortices become, at first, more elongated, and at sufficiently large $\gamma$, develop a large $m=3$ component.

Table 3.3d

mu $=-0.6$

$\begin{array}{lccccccc}\text { Gamma } & \mathrm{V} & \mathrm{V} / \mathrm{Vpv} & \text { Area } & \text { Area } / \mathrm{AO} & \mathrm{dc} & \text { Lambda } & R \\ 0.010 & 0.00003 & 0.82732 & 2.01062 & 1.00000 & 0.40000 & 0.99898 & 0.80000 \\ 1.000 & 0.03483 & 0.41007 & 2.11451 & 1.05167 & 0.39751 & 0.95075 & 0.82041 \\ 1.500 & 0.04916 & 0.32420 & 2.30427 & 1.14605 & 0.38721 & 0.87350 & 0.85643 \\ 1.750 & 0.05422 & 0.28300 & 2.45949 & 1.22325 & 0.37216 & 0.82094 & 0.88480 \\ 2.000 & 0.05466 & 0.23191 & 2.64248 & 1.31426 & 0.33248 & 0.76968 & 0.91713 \\ 2.100 & 0.05008 & 0.20105 & 2.68178 & 1.33381 & 0.29300 & 0.75720 & 0.92392 \\ 2.200 & 0.03766 & 0.15547 & 2.60890 & 1.29756 & 0.21663 & 0.74390 & 0.91128 \\ 2.300 & 0.02369 & 0.11335 & 2.45744 & 1.22223 & 0.13604 & 0.70634 & 0.88444 \\ 2.400 & 0.01397 & 0.08495 & 2.31454 & 1.15116 & 0.07952 & 0.66797 & 0.85834 \\ 2.500 & 0.00724 & 0.06440 & 2.17877 & 1.08363 & 0.04038 & 0.63696 & 0.83278 \\ 2.600 & 0.00251 & 0.04701 & 2.04461 & 1.01691 & 0.01335 & 0.61177 & 0.80673 \\ 2.700 & -0.00083 & 0.03387 & 1.90914 & 0.94953 & 0.00478 & 0.58999 & 0.77955 \\ 2.800 & -0.00314 & 0.05233 & 1.77059 & 0.88062 & 0.01615 & 0.56941 & 0.75073 \\ 2.900 & -0.00476 & 0.06491 & 1.62553 & 0.80847 & 0.02244 & 0.54761 & 0.71932\end{array}$




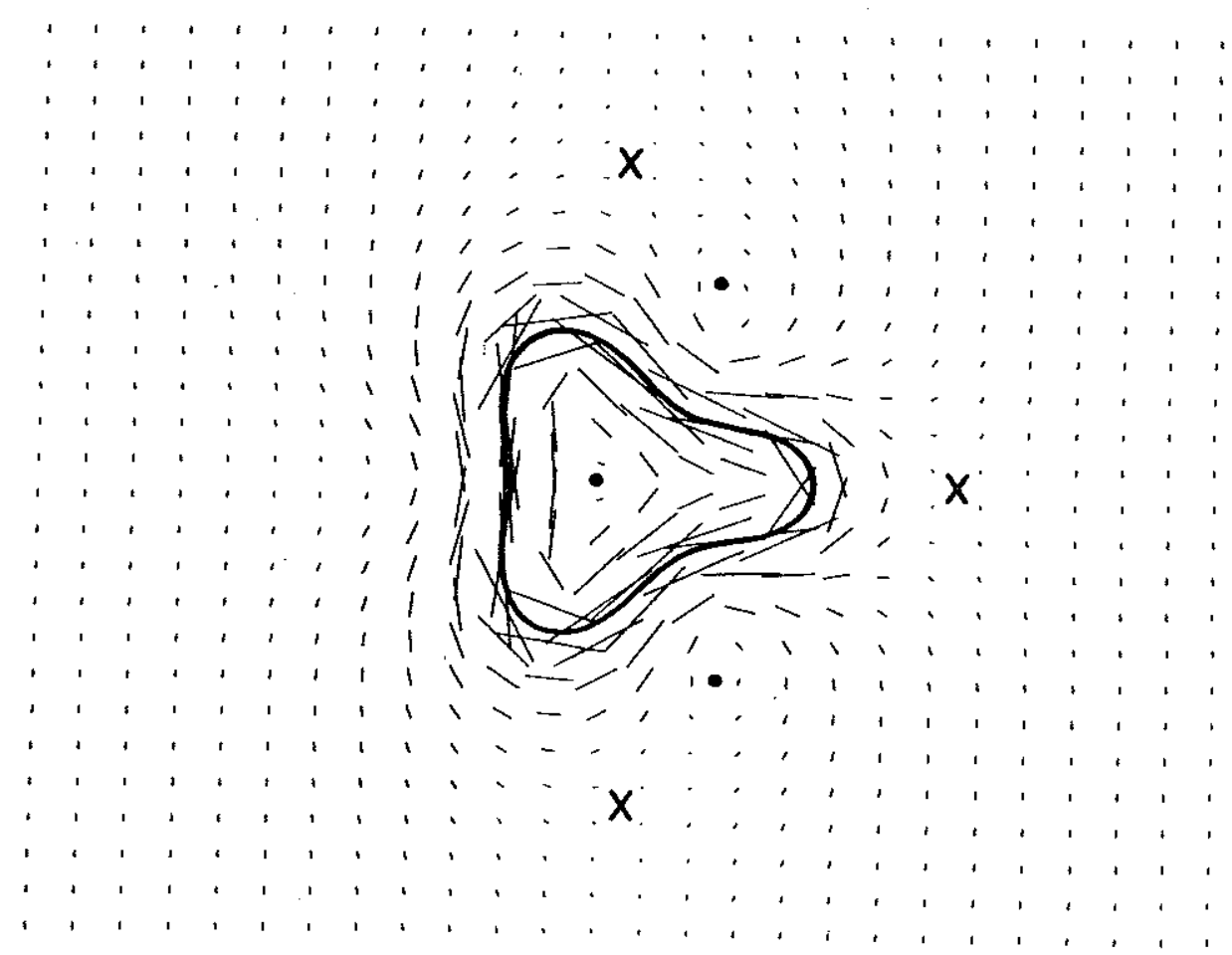

FIGURE 3.30. The velocity field in the upper layer and in the cotranslating frame for the dipole at $\mu=-0.6$ and $\gamma=2.90$. Note the presence of 3 centers and 3 saddles. 


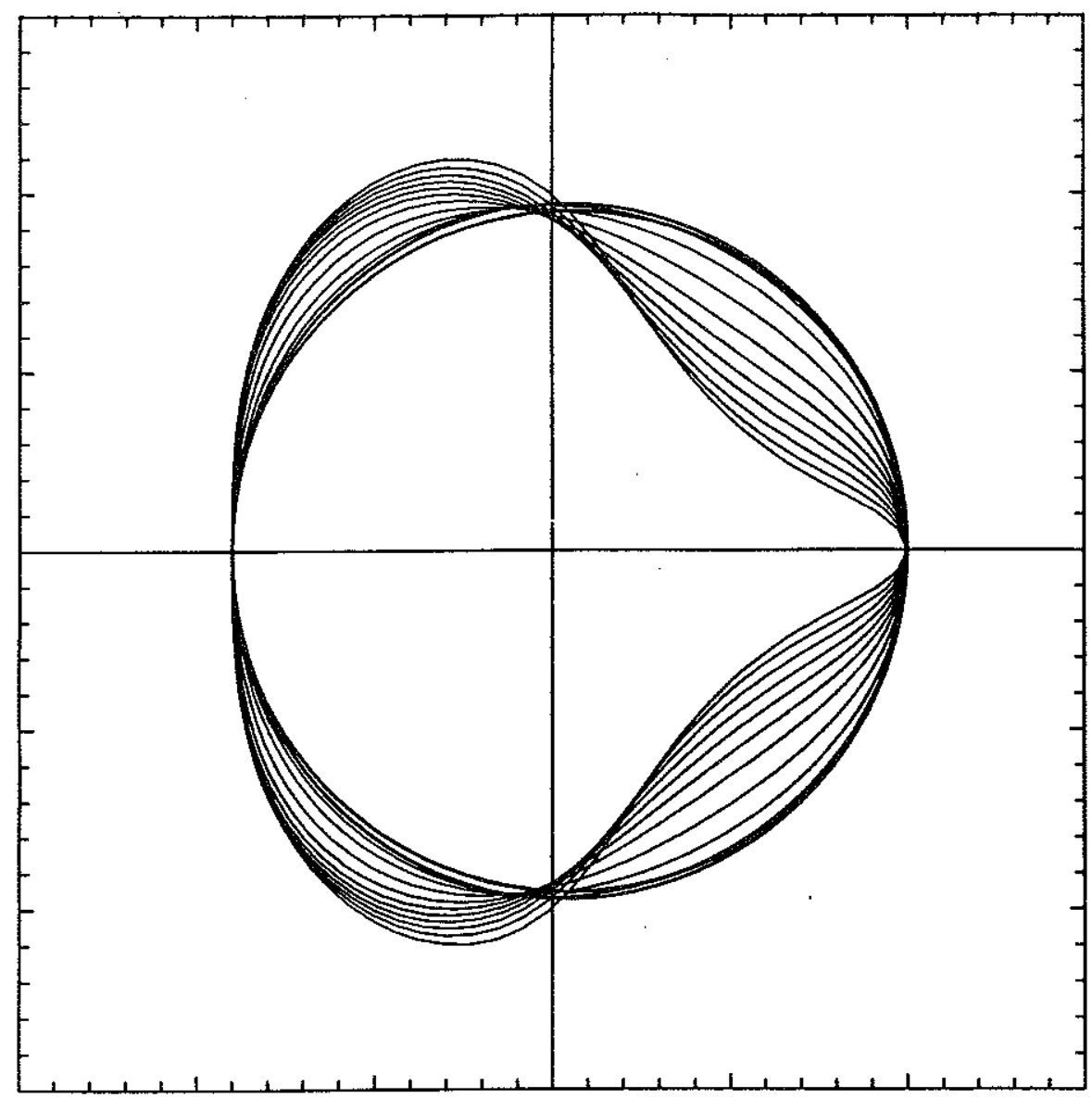

FIGURE 3.31. Two-layer dipoles for $\mu=-0.9$ (only the upper layer vortex is shown). The values of $\gamma$ are given in the table below. As $\gamma$ increases the vortices develop high curvatures and a cusp appears near $\mathrm{X}_{B}$.

Table $3.3 e$

$$
\text { mu }=-0.9
$$

$\begin{array}{cccccccc}\text { Gamma } & \mathrm{V} & \mathrm{V} / \mathrm{Vpv} & \text { Area } & \text { Area/AO } & \text { dc } & \text { Lambda } & \mathrm{R} \\ 0.010 & 0.00001 & 0.65069 & 2.83529 & 1.00000 & 0.10000 & 0.99898 & 0.95000 \\ 1.000 & 0.01041 & 0.17816 & 2.84624 & 1.00386 & 0.09995 & 0.99603 & 0.95183 \\ 2.000 & 0.01552 & 0.09060 & 2.90517 & 1.02465 & 0.09461 & 0.97594 & 0.96164 \\ 2.050 & 0.01502 & 0.08636 & 2.90690 & 1.02526 & 0.09048 & 0.97458 & 0.96192 \\ 2.100 & 0.01206 & 0.07581 & 2.89494 & 1.02104 & 0.07198 & 0.96620 & 0.95994 \\ 2.120 & 0.00515 & 0.05450 & 2.85889 & 1.00832 & 0.03077 & 0.92944 & 0.95395 \\ 2.150 & -0.00574 & 0.05591 & 2.80343 & 0.98876 & 0.03468 & 0.86777 & 0.94465 \\ 2.200 & -0.01725 & 0.08812 & 2.74503 & 0.96817 & 0.10443 & 0.80820 & 0.93476 \\ 2.250 & -0.02597 & 0.11061 & 2.69894 & 0.95191 & 0.15719 & 0.76748 & 0.92688 \\ 2.300 & -0.03338 & 0.13023 & 2.65841 & 0.93761 & 0.20151 & 0.73599 & 0.91989 \\ 2.350 & -0.03998 & 0.14851 & 2.62293 & 0.92510 & 0.24020 & 0.71053 & 0.91373 \\ 2.400 & -0.04601 & 0.16577 & 2.59522 & 0.91533 & 0.27420 & 0.68962 & 0.90889 \\ 2.450 & -0.05152 & 0.18144 & 2.58127 & 0.91041 & 0.30328 & 0.67233 & 0.90645 \\ 2.500 & -0.05641 & 0.19404 & 2.58826 & 0.91287 & 0.32632 & 0.65768 & 0.90767\end{array}$




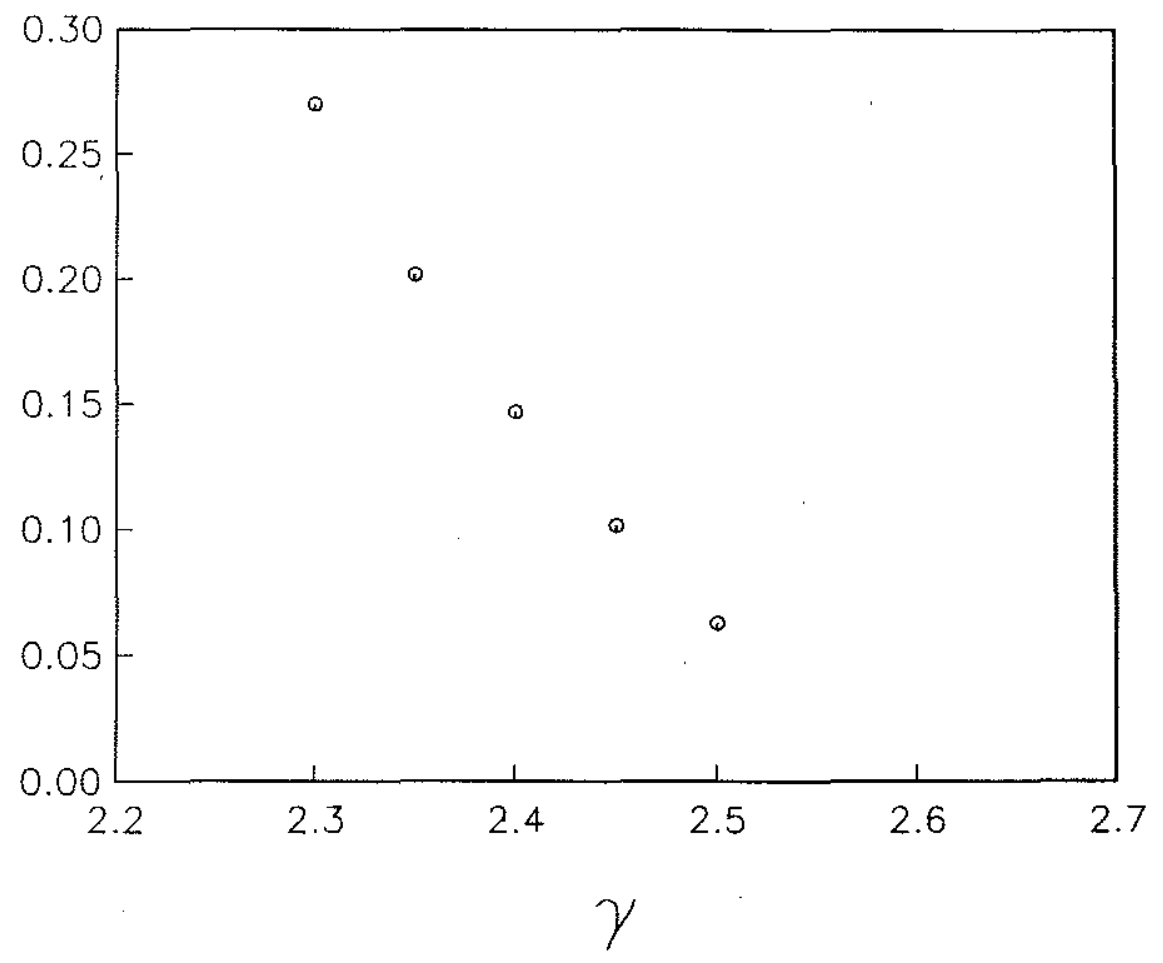

FIGURE 3.32. Plot of the distance between the critical pont $S_{0}$ and the contour as a function of $\gamma$. There is a strong suggestion that a limiting $V$-state exists at finite $\gamma$. 
Table $3.4 \mathrm{a}$

\begin{tabular}{|c|c|c|c|c|c|c|c|c|}
\hline Gamma & V & $\mathrm{V} / \mathrm{Vpv}$ & Area & Area / AO & $d c$ & Lambda & $\mathrm{R}$ & nit \\
\hline $\begin{array}{l}0.010 \\
1.000 \\
2.000 \\
2.050 \\
2.100 \\
2.120 \\
2.150 \\
2.200 \\
2.250 \\
2.300 \\
2.350 \\
2.400 \\
2.450 \\
2.500\end{array}$ & $\begin{array}{r}0.00001 \\
0.01041 \\
0.01552 \\
0.01502 \\
0.01206 \\
0.00515 \\
-0.00574 \\
-0.01725 \\
-0.02597 \\
-0.03338 \\
-0.03998 \\
-0.04601 \\
-0.05152 \\
-0.05641\end{array}$ & $\begin{array}{l}0.65069 \\
0.17816 \\
0.09060 \\
0.08636 \\
0.07581 \\
0.05450 \\
0.05591 \\
0.08812 \\
0.11061 \\
0.13023 \\
0.14851 \\
0.16577 \\
0.18144 \\
0.19404\end{array}$ & $\begin{array}{l}2.83529 \\
2.84624 \\
2.90517 \\
2.90690 \\
2.89494 \\
2.85889 \\
2.80343 \\
2.74503 \\
2.69894 \\
2.65841 \\
2.62293 \\
2.59522 \\
2.58127 \\
2.58826\end{array}$ & $\begin{array}{l}1.00000 \\
1.00386 \\
1.02465 \\
1.02526 \\
1.02104 \\
1.00832 \\
0.98876 \\
0.96817 \\
0.95191 \\
0.93761 \\
0.92510 \\
0.91533 \\
0.91041 \\
0.91287\end{array}$ & $\begin{array}{l}0.10000 \\
0.09995 \\
0.09461 \\
0.09048 \\
0.07198 \\
0.03077 \\
0.03468 \\
0.10443 \\
0.15719 \\
0.20151 \\
0.24020 \\
0.27420 \\
0.30328 \\
0.32632\end{array}$ & $\begin{array}{l}0.99898 \\
0.99603 \\
0.97594 \\
0.97458 \\
0.96620 \\
0.92944 \\
0.86777 \\
0.80820 \\
0.76748 \\
0.73599 \\
0.71053 \\
0.68962 \\
0.67233 \\
0.65768\end{array}$ & $\begin{array}{l}0.95000 \\
0.95183 \\
0.96164 \\
0.96192 \\
0.95994 \\
0.95395 \\
0.94465 \\
0.93476 \\
0.92688 \\
0.91989 \\
0.91373 \\
0.90889 \\
0.90645 \\
0.90767\end{array}$ & $\begin{array}{r}1 \\
23 \\
105 \\
127 \\
398 \\
600 \\
265 \\
133 \\
94 \\
83 \\
80 \\
78 \\
83 \\
224\end{array}$ \\
\hline
\end{tabular}

Table $3.4 \mathrm{~b}$

$m u=0.9$

$\begin{array}{rcccccccr}\text { Gamna } & V & V / V p v & \text { Area } & \text { Area/AO } & \text { dc } & \text { Lambda } & \text { R } & \text { nit } \\ 0.010 & 0.00001 & 0.65069 & 2.83529 & 1.00000 & 0.10000 & 0.99898 & 0.95000 & 1 \\ 1.000 & 0.01041 & 0.17816 & 2.84624 & 1.00386 & 0.09995 & 0.99603 & 0.95183 & 23 \\ 2.000 & 0.01552 & 0.09060 & 2.90518 & 1.02465 & 0.09461 & 0.97594 & 0.96164 & 106 \\ 2.050 & 0.01502 & 0.08636 & 2.90690 & 1.02526 & 0.09048 & 0.97458 & 0.96192 & 166 \\ 2.100 & 0.01206 & 0.07581 & 2.89495 & 1.02104 & 0.07198 & 0.96620 & 0.95994 & 448 \\ 2.120 & 0.00515 & 0.05450 & 2.85889 & 1.00832 & 0.03077 & 0.92944 & 0.95395 & 727 \\ 2.150 & -0.00574 & -0.05591 & 2.80343 & 0.98876 & 0.03468 & 0.86777 & 0.94465 & 415 \\ 2.200 & -0.01725 & -0.08812 & 2.74503 & 0.96817 & 0.10443 & 0.80821 & 0.93476 & 248 \\ 2.250 & -0.02597 & -0.11061 & 2.69894 & 0.95191 & 0.15719 & 0.76748 & 0.92688 & 190 \\ 2.300 & -0.03338 & -0.13023 & 2.65841 & 0.93761 & 0.20151 & 0.73599 & 0.91989 & 166 \\ 2.350 & -0.03998 & -0.14851 & 2.62293 & 0.92510 & 0.24020 & 0.71053 & 0.91373 & 154 \\ 2.400 & -0.04601 & -0.16577 & 2.59521 & 0.91533 & 0.27420 & 0.68962 & 0.90889 & 146 \\ 2.450 & -0.05152 & -0.18145 & 2.58122 & 0.91039 & 0.30330 & 0.67231 & 0.90644 & 149 \\ 2.500 & -0.05642 & -0.19407 & 2.58808 & 0.91281 & 0.32636 & 0.65767 & 0.90764 & 306\end{array}$




\section{Critical Points and Filamentation}

Se non è vero, è molto ben trovato.

\section{Giordano Bruno}

Filamentation, the formation of extremely thin threads of vorticity in the vicinity of otherwise coherent vortex structures, has come to be recognized, in recent years, as one of the most common phenomena in the evolution of twodimensional vorticity fields. Originally observed by Deem and Zabusky (1978a and b), it was for the first time revealed in all its beauty by the very high resolution contour dynamical calculations of Dritschel $(1988,1988 \mathrm{a}, 1988 \mathrm{~b})$ but has, until now, eluded simple explanations.

To illustrate the complexity of this phenomenon we reprint in Figure 4.1 the evolution of a perturbed Kirchhoff ellipse, calculated with a contour surgery algorithm by Dritschel (1988). Notice how very thin filaments are repeatedly generated leading to an extremely complicated convolution of the contour from a very simple initial condition.

It is almost impossible to contemplate this without being reminded of the complex behavior exhibited by the solutions of nonlinear dynamical system, which have been the object of much study in the last half century. Among the many discoveries of modern dynamical system theory is the importance of what have come to be called "Smale horseshoes", whereby a volume of phase space is repeatedly stretched and folded into a fabulously contorted shape. These are known to occur, in particular, near the intersection of the stable and unstable invariant manifolds at a hyperbolic fixed point.

That these ideas from dynamical system theory can be directly related to the evolution of two-dimensional inviscid incompressible flows becomes apparent upon consideration of the simple fact that the familiar expressions for the velocity in

If it is not true, it is still a nice idea. 
terms of the streamfunction, i.e.

$$
\mathrm{u}=\mathrm{dx} / \mathrm{dt}=-\psi_{\mathrm{y}}, \quad \text { and } \quad \mathrm{v}=\mathrm{dy} / \mathrm{dt}=\psi_{\mathrm{x}}
$$

are in fact Hamilton's equations, in which the streamfunction plays the role of the Hamiltonian. Thus, among others, a direct analogy can be made between the stagnation points of the flow and the hyperbolic points mentioned above (see, for instance Wiggins 1988, Aref 1988 and references therein).

The object of this chapter is thus very simple: we intend to make manifest how the process of filamentation can be directly attributed to the presence of a critical (hyperbolic) point - in a comovingt reference frame - which comes into contact with the vorticity distribution. We will show that a careful analysis of the geometry of the comoving streamfunction, and in particular the location of the critical points, can in fact be used to predict the time at which filamentation will occur.

We must, however, start from the simplest example, which we treat in the next section (4.1), of the geometry of the corotating streamfunction of a perturbed circular vortex. We then proceed to the analysis of a perturbed Kirchhoff ellipse, which provides the simplest and clearest instance for the observation of filamentation and its connection to the presence of the critical points of the flow (Section 4.2). We then extend these considerations from the Euler equations to the equivalent barotropic model, and show how the absence of filamentation and axisymmetrization at large $\gamma$ discovered in Chapter 2 , can again be understood from a geometrical analysis of the corotating fields (Section 4.3).

\subsection{The Linearly Perturbed Rankine Vortex $\ddagger$}

Let us start by considering the simplest possible two-dimensional vorticity structure: the Rankine vortex, namely a circular patch of constant vorticity embedded in an infinite irrotational flow. Without loss of generality we choose the vorticity to have value 1 inside the radius $R$. The streamfunction $\psi$ associated with this vortex is found by solving the simple system:

\footnotetext{
$\dagger$ It is obvious that the general considerations of this chapter can be applied without qualitative differences to both translating and rotating configurations.

\# Throughout this section the symbols $r$ and $\theta$ (or $\vartheta$ ) designate standard polar coordinates in the $x-y$ plane.
} 


$$
\begin{array}{lll}
\nabla^{2} \psi_{i}=1 & \text { for } & r<R \\
\nabla^{2} \psi_{0}=0 & \text { for } & r>R
\end{array}
$$

together with the boundary conditions that $\psi$ and $\psi_{\mathrm{r}}$ be continuous at $\mathrm{r}=\mathrm{R}$. The solution is easily found to be:

$$
\begin{array}{lll}
\psi_{i}=(1 / 4)\left[r^{2}-R^{2}\right] & \text { for } & r<R \\
\psi_{0}=(1 / 2) R^{2} \log [r / R] & \text { for } & r>R
\end{array}
$$

The stability of the solution (4.1) was investigated by Lord Kelvin more than a hundred years ago (1880), and the results are well known (cf. for instance Lamb, 1945). If the boundary is displaced by an infinitesimal periodic perturbation of mode $\mathrm{m}$ so that its location is given by the expression:

$$
r=R+\varepsilon \eta(\theta, t)
$$

where

$$
\eta(\theta, t) \equiv(1 / 2) R^{-1} \cos (m \theta-\omega t)
$$

and $\varepsilon<<1$, the streamfunction associated with the perturbation is found to be:

$$
\begin{aligned}
\varphi_{i} & =-\varepsilon(r / R)^{2} \cos (m \theta-\omega t) \\
\varphi_{o} & =-\varepsilon(R / r)^{2} \cos (m \theta-\omega t)
\end{aligned}
$$

and the dispersion relation is given by:

$$
\omega=(m-1) / 2
$$

We now wish to investigate the geometry of the streamfunction in a frame that rotates with the same angular velocity $\Omega=\omega / \mathrm{m}$ as the perturbation. The total corotating streamfunction $\Psi_{c}$ is obtained from the inertial one $\Psi=\psi+\varphi$ by subtracting a term (1/2) $\Omega \mathrm{r}^{2}$ and changing the angular variable to $\vartheta=\theta-\Omega$ t. For simplicity let's consider the case $\mathrm{m}=2$ for which $\Omega=1 / 4$. The total exterior streamfunction in the corotating frame is then given by:

$$
\Psi_{c}=(1 / 2) R^{2} \log (r / R)-\varepsilon(R / r)^{2} \cos (2 \vartheta)-(1 / 2) \Omega r^{2}
$$

and the boundary of the vortex is located at:

$$
r=R+\varepsilon(1 / 2 R) \cos (2 \vartheta)
$$


We now proceed to the exploration of the geometry of $\Psi_{\mathrm{c}}$. First we wish to find the critical points - located at $\left(r_{c}, \vartheta_{c}\right)$ - which are found by solving:

$$
\partial_{\mathrm{r}} \Psi_{\mathrm{c}}\left(r_{\mathrm{c}}, \vartheta_{\mathrm{c}}\right)=0 \quad \text { and } \quad \partial_{\vartheta} \Psi_{\mathrm{c}}\left(\mathrm{r}_{\mathrm{c}}, \vartheta_{\mathrm{c}}\right)=0
$$

The second equation immediately gives $\vartheta_{c}=0, \pi / 2, \pi$ and $3 \pi / 2$ but, from (4.4) it easy to see that the hyperbolic critical points are located at 0 and $\pi$ (the significance of the solutions $\pi / 2$ and $3 \pi / 2$ will become clear in moment). Since $\varepsilon$ is infinitesimal we then write:

$$
r_{c}=r_{c}(0)+\varepsilon r_{c}^{(1)}+O\left(\varepsilon^{2}\right)
$$

and substituting this into the first equation of (4.5) we easily find:

$$
r_{c}(0)=\sqrt{ } 2 R
$$

This is an important result because it shows that, even when the perturbation is infinitesimal, the critical point of the corotating streamfunction is located at an $O(1)$ distance from the contour. This distance is equal to $(\sqrt{ } 2-1) \approx .41$ times the radius of the vortex itself. It is also easy to show that the $O(\varepsilon)$ correction to the location of the critical point is given by:

$$
r_{c}(1)=\sqrt{ } 2 / R^{2}
$$

The next task is to find the separatrices that emanate from these critical points. Since we know that they are very nearly circles of radius $r_{c}(0)$, we let them be defined by the equation:

$$
r_{s}(\vartheta)=\sqrt{ } 2 R+\varepsilon^{\beta} \rho_{s}(\vartheta)
$$

The function $\rho_{s}$ and the exponent $\beta$ are determined by requiring that $\Psi_{c}$ along the separatrices have the same value as at the critical points, i.e.:

$$
\Psi_{c}\left[r_{s}(\vartheta), \vartheta\right]=\Psi_{c}\left[r_{c}, \vartheta_{c}=0 \text { or } \pi\right]
$$

Substitution of (4.7) into (4.8) shows that dominant balance at order $\varepsilon$ can only be achieved if $\beta=1 / 2$, and yields the expression for the separatrices:

$$
\rho_{s}(\vartheta)= \pm \sqrt{ } 2 \sin \vartheta
$$

It easy to show from (4.7) and (4.9) that the separatrices, in this simplest case, are actually exact circles of radius $\sqrt{2} R$ whose centers are displaced above and below 
the $x$-axis by an amont $O\left(\varepsilon^{1 / 2}\right)$, which is much larger than the $O(\varepsilon)$ displacement of the perturbed vortex boundary given by (4.4).

This geometry is illustrated in Figure 4.2. The description of the corotating streamfunction can be completed by calculating the position of the centers of the ghost vortices (the regions enclosed by the separatrices) located at $\left(r_{g}, \vartheta_{g}\right)$, which are found by solving:

$$
\partial_{r} \Psi_{c}\left(r_{g}, \vartheta_{g}\right)=0 \quad \text { and } \quad \partial_{\vartheta} \Psi_{c}\left(r_{g}, \vartheta_{g}\right)=0
$$

Since $\vartheta_{g}=\pi / 2$ and $3 \pi / 2$, and $r_{g}$ can only be $O(\varepsilon)$ away from $\sqrt{ } 2 R$, letting:

$$
r_{g}=\sqrt{ } 2 R+\varepsilon \rho_{g}+O\left(\varepsilon^{2}\right)
$$

and substituting into $(4.10)$ yields:

$$
\rho_{g}=-2 / R
$$

The centers of the ghost vortices therefore reside inside the circle of radius $\sqrt{2} \mathrm{R}$. It is important to note that in the limit $\varepsilon \rightarrow 0$ the two separatrices coalesce on this circle and the ghost vortices disappear. The unperturbed Rankine vortex possesses therefore a "critical circle" located at $\sqrt{2} \mathrm{R}$ with respect to $\mathrm{m}=2$ perturbations.

As a matter of fact, the Rankine vortex possesses a countable infinity of critical circles, one for each value of the angular wavenumber $m$ of the linear eigenmodes. Using the dispersion relation (4.2) it is easy to show that the critical circle for mode $m$ is located at

$$
r_{c, m}=\left[\frac{m}{m-1}\right]^{1 / 2} R
$$

What really matters is that in the limit $m \rightarrow \infty$ the distance between the vortex boundary and the critical circle goes to zero. Thus, in a way totally analogous to the $m=2$ case that we have just illustrated, an infinitesimal perturbation of mode $m$ will give rise to $m$ critical points located a distance $r_{c, m}-R$ from the boundary, and $m$ ghost vortices.

This simple fact is at the root of the recent discovery of Dritschel (1988a) regarding the filamentation of the Rankine vortex. After perturbing a Rankine vortex with a small Gaussian "bump" on an otherwise circular contour, Dritschel observed that, no matter how small the amplitude of the initial perturbation, filaments of vorticity were eventually expelled, leading to an extremely complex evolution of the 
boundary shape. This has seemed to be difficult to reconcile with the linear analysis which shows (cf. equation 4.2) that all modes are stable.

It is our belief, for which the next section will provide much evidence, that the discovery of Dritschel can be easily understood from the presence of the above mentioned critical points that, once in contact with the vortex, actually extract the vorticity filaments. Since the initially Gaussian perturbation used by Dritschel can be considered to contain even the highest modes, which, as we have shown, have associated critical points which are extremely close to the boundary of the vortex, it is not suprising that, given enough time, these modes would grow sufficiently through the nonlinear interactions to make the vortex boundary collide with a critical point, thereby leading to the formation of a filament.

We believe that, in Dritschel's experiment, the nonlinearity is a fundamental ingredient that, by mediating the energy exchange between the modes, allows the higher ones to gain sufficient amplitude to come in contact with their associated critical point, and thus to filament. Indeed if the evolution of the perturbation were arbitrarily made perfectly linear the modes would simply propagate around the circular vortex boundary with the dispersive angular velocity $\Omega$ but without interacting, and the initial Gaussian bump would simply recur every $t=4 \pi \dagger$.

The role of nonlinearity is thus crucial to the filamentation of the linearly stable Rankine vortex. We have chosen to consider instead the filamentation of the Kirchhoff ellipse, which presents the advantage of possessing unstable normal modes. We are going to show that their growth leading to the coincidence of a critical point with the vortex is the essential mechanism for filamentation. The reason for studying this apparently more complicated problem (most of the analysis has to be carried out in elliptical coordinates) is that it is possible to isolate the filamentation process from the simple linear growth of an unstable eigenmode. Because the nonlinear interactions are not playing as great a role as in the circular problem, the comparison between linear theory and numerical experiments can be meaningfully made and is found to be surprisingly good.

t It is simple to show that $\eta(\theta, t+4 \pi)=\eta(\theta, t)$ if $\omega$ is given by $(4.2)$. 


\subsection{The Filamentation of Kirchhoff Ellipses}

The Kirchhoff vortex, an elliptical region of constant vorticity q surrounded by irrotational fluid and rotating without change in shape with angular velocity $\Omega_{k}$ given by

$$
\Omega_{\mathrm{k}}=\mathrm{q} \frac{\mathrm{ab}}{(\mathrm{a}+\mathrm{b})^{2}}
$$

where $a$ and $b$ are the major and minor axes of the ellipse, is an exact nonlinear solution of the Euler equations. The linear stability of this vortex was analyzed by Love (1893), who showed that infinitesimal perturbations will grow in time when the ratio $a / b$ is greater than 3 .

In a frame of reference rotating with constant angular velocity $\Omega_{\mathrm{k}}$ the topology of the streamfunction of an unperturbed Kirchhoff ellipse is qualitatively identical to the one produced by an $\mathrm{m}=2$ perturbation on a circular vortex shown in Figure 4.2. It is characterized by two critical points located outside the vortex along the major axis and two ghost vortices centered on the $y$-axis above and below the ellipse.

What we intend to show in this section is the following: when a small unstable mode is added to the ellipse at $t=0$, it grows according to linear theory until the boundary of the perturbed ellipse comes into contact with one or more critical points of the corotating streamfunction, after which filaments are extracted. Because the growth of the perturbation is an essentially linear phenomenon, the time at which the filament appears can actually be predicted analytically, and the agreement with numerical initial value calculations is found to be good.

We first present the theory which leads to an analytic expression for the time of filamentation, from now on referred to as $\mathrm{t}_{f}$, which we define as the time when one or more critical points become coincident with the vortex boundary. Without loss of generality, we can choose the vorticity $q$ of the ellipse to be identically equal to 1 , and its area to be exactly $\pi$ (i.e. $a b=1$ ). The only parameter describing the ellipse is then its aspect ratio $\lambda \equiv b / a$ (note that $0<\lambda<1$ ) so that:

$$
\Omega_{\mathrm{k}}=\frac{\lambda}{(1+\lambda)^{2}} \quad \mathrm{a}=\frac{1}{\sqrt{\lambda}} \quad \mathrm{b}=\sqrt{ } \lambda
$$


The natural coordinate system for the problem is the elliptical one, where the elliptical radius $\rho$ and the elliptical angle $\vartheta$ are implicitly defined in terms of the cartesian coordinates $(x, y)$ by the relations:

$$
x=c \cosh \rho \cos \vartheta \text { and } y=c \sinh \rho \sin \vartheta
$$

and $c \equiv\left(a^{2}-b^{2}\right)^{1 / 2}$. In this coordinate system, the equation for the ellipse of aspect ratio $\lambda$ simply reduces to:

$$
\rho=\rho_{0}=\tanh ^{-1} \lambda
$$

The streamfunction $\psi$ associated with the Kirchhoff solution is given by:

$$
\begin{array}{lll}
\psi_{0}=(1 / 2) \rho+(1 / 4) e^{-2 \rho} \cos 2 \vartheta & \text { for } & \rho>\rho_{0} \\
\psi_{i}=(1 / 2)(1+\lambda)^{-1}\left(\lambda x^{2}+y^{2}\right) & \text { for } & \rho<\rho_{0}
\end{array}
$$

We now want to investigate the geometry of the streamfunction associated with the Kirchhoff solution in a frame rotating with angular velocity $\Omega_{\mathrm{k}}$. As for the perturbed Rankine vortex, the whole analysis can be done exactly.

Let the exterior corotating streamfunction be defined by

$$
\begin{aligned}
\psi_{c} & =\psi_{o}-(1 / 2) \Omega_{k}\left(x^{2}+y^{2}\right) \\
& =\psi_{0}-(1 / 4) \Omega_{k} c^{2}(\cosh 2 \rho+\cos 2 \vartheta)
\end{aligned}
$$

As was shown in the previous section, the corotating streamfunction of an elliptical vortex is qualitatively given by the sketch in Figure 4.2; we now wish to determine the exact position $\left(\rho_{c}, \vartheta_{c}\right)$ of the critical points. They are found by solving the system:

$$
\partial_{\rho} \psi_{c}\left(\rho_{c}, \vartheta_{c}\right)=0 \quad \text { and } \quad \partial_{\vartheta} \psi_{c}\left(\rho_{c}, \vartheta_{c}\right)=0
$$

Substitution of (4.16) into (4.17) yields the system:

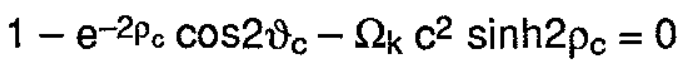

$$
\begin{aligned}
& \left(\Omega_{k} c^{2}-e^{-2 \rho}\right) \sin 2 \vartheta_{c}=0
\end{aligned}
$$


The second of these equations yields $\vartheta_{C}=0$ or $\pi^{\dagger}$ (the solutions $\pi / 2$ and $3 \pi / 2$ are the centers of the ghost vortices $\ddagger$ ), and upon substitution into the first one, the equation to be solved for $\rho_{c}$ becomes:

$$
e^{-2 \rho_{c}}+\Omega_{k} c^{2} \sinh 2 \rho_{c}=1
$$

from which the exact expression for $\rho_{c}$ is easily found to be:

$$
\rho_{c}=(1 / 2) \log \left(\frac{1+3 \lambda}{1-\lambda}\right)
$$

Recalling that the major axis of the ellipse extends along the positive $x$-axis to $\mathrm{x}=\mathrm{a}=\mathrm{c}$ cosh $\rho_{0}$, the interesting quantity to consider is the ratio of the $\mathrm{x}$ coordinate of the critical point $x_{c}$ to the length of the major axis $a$. This is a function of the aspect ratio $\lambda$ alone, and is easily shown to be:

$$
\frac{x_{c}}{a}(\lambda)=\frac{(1+\lambda)^{3 / 2}}{(1+3 \lambda)^{1 / 2}}
$$

We have plotted this quantity versus $\lambda$ in Figure 4.3. Three important points have to be made. First, as $\lambda \rightarrow 1$ it is easy to show from (4.19) that $x_{c} \rightarrow \sqrt{2} a$, so that the circular result (4.6) is recovered in that limit. Second, it is apparent upon inspection of (4.19) that the critical point becomes infinitely close to the ellipse as the aspect ratio becomes infinitely large, i.e. $x_{c} \rightarrow a$ as $\lambda \rightarrow 0$. This would suggest that very elongated ellipses should filament faster than more circular ones.

Third, and most importantly, notice that the curve of Figure 4.3 is perfectly smooth at $\lambda=1 / 3$, the linear stability threshold. The point we wish to stress is that there is no connection between the linear stability of the Kirchhoff ellipse and the existence and position of the critical points. A simple way to understand why this is so, is to recall that the validity of a linear analysis rests in its dealing with infinitesimally small amplitudes. Thus as long as the critical point is not exactly on

† Note that here $\vartheta$ is the elliptic angle. However, $\vartheta=0$ (and $\pi$ ) correspond to the portion of the positive (and, respectively, negative) $x$-axis for which $|x|>c$. Recall that the concentration points of the elliptical coordinate system are located at $x= \pm c$ and $y=0$.

$\neq$ For the sake of completeness we note that it can easily be shown that the centers of the ghost vortices are located at $\vartheta_{\mathrm{g}}=\pi / 2$ and $3 \pi / 2$, and at the elliptical radius:

$$
\rho_{g}=\log \left(\frac{3+\lambda}{1-\lambda}\right)
$$


the contour of the unperturbed vortex, the linear stability analysis will show no signs of its presence.

[In Addendum B we present a simple example to illustrate this point a contrario. We show there that the stability of a 2-contour Rankine vortex is in some limits connected with the position of the critical points.]

For a single-contour vortex (i.e. one with a unique discontinuity in the vorticity field), however, the filamentation process is a separate phenomenon from the linear stabilty, as is evidenced by the fact that stable Rankine vortices are also known to filament. In reexamining Figure 4.1, it should then be clear that the breakup of a perturbed ellipse takes place in two quite distinct phases. At first (until about $\mathrm{t}=6$ ) the unstable perturbation grows in amplitude and the dynamics of this evolution are well captured by the linear stability analysis (at least when the initial amplitudes are small, and $\lambda$ is not too large).

When the amplitude has grown sufficiently that the vortex is brought in contact with the critical point, a filament is stripped away from the vortex (cf. the frame $t=8$ of Figure 4.1). The nonlinear evolution that ensues is of course too complicated to be described analytically. However a careful application of the linear theory allows us to predict the time at which the filament will appear.

We start by sketching the linear stability theory of Love (1893). We define the total streamfunction $\Psi \equiv \psi+\varphi$, where $\varphi$ is the perturbation of elliptical angular wavenumber $m$ which, following Love, we write in the form:

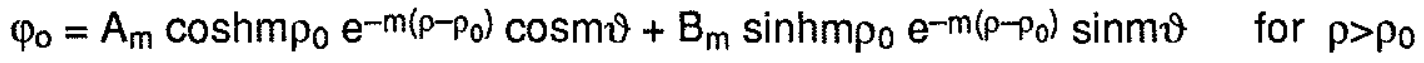

$$
\begin{aligned}
& \varphi_{i}=A_{m} \operatorname{coshm} \rho \cos m \vartheta+B_{m} \operatorname{sinhm} \rho \sin m \vartheta \\
& \text { for } \rho<\rho_{0}
\end{aligned}
$$

where the $A_{m}$ and $B m$ are function of time alone. This choice automatically insures continuity of $\partial_{\vartheta} \varphi$ at the vortex boundary. The equation for the perturbed vortex boundary itself is chosen to bet :

$$
\rho_{v}(\vartheta)=\rho_{0}+h_{0}^{2}\left(\alpha_{m} \cos m \vartheta+\beta_{m} \sin m \vartheta\right)
$$

where

$$
\mathrm{h}_{0 .}^{-2}=\lambda^{-1} \cos ^{2} \vartheta+\lambda \sin ^{2} \vartheta
$$

\footnotetext{
† We note incidentally that it is this clever choice of $(4.20)$ that allows the decoupling of the different angular modes in this problem. The factor $\left(h_{0}\right)^{2}$ is necessary to perturb the vortex without altering its total circulation.
} 
Requiring continuity in $\partial_{\rho} \varphi$ at the boundary yields the identities:

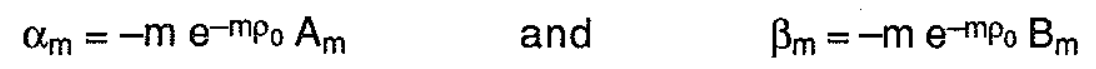

The last condition to be imposed is that the fluid inside the ellipse remain inside at all times. This condition yields the evolution equations for the amplitudes $A_{m}$ and $B_{m}$ as functions of time, which take the simple form:

$$
\begin{aligned}
& \frac{d}{d t} A_{m}=+\left[\frac{m \lambda}{(1+\lambda)^{2}}-e^{-m \rho_{0}} \sinh m \rho_{0}\right] B_{m} \\
& \frac{d}{d t} B_{m}=-\left[\frac{m \lambda}{(1+\lambda)^{2}}-e^{-m \rho_{0} \cosh m \rho_{0}}\right] A_{m}
\end{aligned}
$$

The last step, yielding the dispersion relation for infinitesimal normal mode perturbations of wavenumber $m$ on the boundary of a Kirchhoff ellipse, is to let $A_{m}$ and $B_{m}$ be proportional to $\exp (-i \sigma t)$. This gives:

$$
\sigma^{2}=(1 / 4)\left(\left[\frac{2 m \lambda}{(1+\lambda)^{2}}\right]^{2}-\left[\frac{1-\lambda}{1+\lambda}\right]^{2 m}\right)
$$

first obtained by Love (1893), who showed that mode 1 is stable, mode 2 is always neutral $\left(\sigma^{2}=0\right)$ and mode 3 becomes unstable for $\lambda>1 / 3$. From (4.20) is it easy to see that, for the unstable modes, $\operatorname{Re}(\sigma)=0$ so that they simply grow in place, without propagating around the ellipse.

We now wish to examine more closely the streamfunction of a perturbed ellipse, and in particular, by considering the frame rotating with angular velocity $\Omega_{k}$, to determine the location of the critical points of the corotating streamfunction for $t>0$. Let us concentrate our attention on the case of an ellipse of aspect ratio $\lambda$ to which we superimpose, at $\mathrm{t}=0$, an unstable infinitesimal perturbation of mode $\mathrm{m}$. We let:

$$
s^{2} \equiv-\sigma^{2}>0
$$

so that the solution of system (4.19) can be written:

$$
\begin{aligned}
& A_{m}(t)=A_{m}^{+} e^{s t}+A_{m}^{-} e^{-s t} \\
& B_{m}(t)=B_{m}^{+} e^{s t}+B_{m}^{-} e^{-s t}
\end{aligned}
$$


Substitution of (4.27) into (4.23) yields the relation:

$$
\mathrm{B}_{\mathrm{m}}^{ \pm}= \pm \chi \mathrm{A}_{\mathrm{m}}^{ \pm}
$$

with

$$
\chi=e^{-m \rho_{0}} \operatorname{coshm} \rho_{0}-\frac{m \lambda}{(1+\lambda)^{2}}
$$

The $A_{m}$ 's are determined by the choice of a perturbation at $t=0$. As was done by Dritschel (1988) for the example shown in Figure 4.1, we choose initial perturbations of the vortex boundary with only the $\operatorname{cosm} \vartheta$ mode present at $t=0$, namely:

$$
\left.\alpha_{m}\right|_{t=0}=\varepsilon \quad \text { and }\left.\quad \beta_{m}\right|_{t=0}=0
$$

where $\varepsilon$ is infinitesimal. Combining (4.22), (4.23), (4.27), (4.28) and (4.30) it is easy to show that this choice implies:

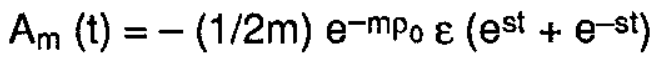

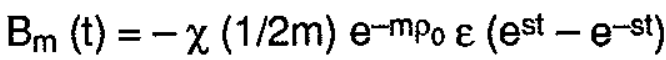

The total exterior streamfunction $\Psi_{0}$ for the evolving perturbed unstable ellipse can then be written as follows:

$$
\begin{aligned}
\Psi_{0} & =\psi_{0}+\varphi_{0} \\
& =(1 / 2) \rho+(1 / 4) \varepsilon^{-2 \rho} \cos 2 \vartheta \\
& -\varepsilon(1 / 2 m) \operatorname{coshm} \rho_{0}\left(e^{s t}+e^{-s t}\right) e^{-m \rho} \cos m \vartheta \\
& -\varepsilon(1 / 2 m) \chi \operatorname{sinhm} \rho_{0}\left(e^{s t}-e^{-s t}\right) e^{-m \rho} \operatorname{sinm} \vartheta
\end{aligned}
$$

The next step is to select a corotating frame, and we are here confronted with what is probably the weakest point of this theory. The crux of the matter is that one cannot precisely define a corotating frame when the vorticity field is not stationary. This choice is thus formally always somewhat arbitrary (cf. Melander et al 1987a) but, in practice, can be quite good. In the present context we proceed by assuming that the perturbation is small enough that the angular velocity of the perturbed vortex has deviated from $\Omega_{\mathrm{k}}$ by only negligible amounts by the time the critical point comes into contact with the vortext.

T The numerical simulations that we will present next provide an excellent a posteriori validation of this assumption. We have found that for small initial perturbations (say $\varepsilon<0.01$ ) the deviation of the angular velocity of the perturbed vortex obtained by fitting ellipses to the vorticity distribution at small time intervals and 
We thus define the corotating exterior total streamfunction $\Psi_{\mathrm{c}}$ as follows:

$$
\begin{aligned}
\Psi_{\mathrm{c}} & \equiv \Psi_{\mathrm{o}}-(1 / 2) \Omega_{\mathrm{k}}\left(\mathrm{x}^{2}+\mathrm{y}^{2}\right) \\
& =\Psi_{\mathrm{o}}-(1 / 4) \Omega_{\mathrm{k}} \mathrm{c}^{2}(\cosh 2 \rho+\cos 2 \vartheta)
\end{aligned}
$$

and determine the positions $\left(\mathrm{P}_{\mathrm{c}}, \Theta_{\mathrm{c}}\right)$ of the critical points by solving the system:

$$
\partial_{\rho} \Psi_{c}\left(P_{c}, \Theta_{c}\right)=0 \quad \text { and } \quad \partial_{\vartheta} \Psi_{c}\left(P_{c}, \Theta_{c}\right)=0
$$

Since we are dealing with linear theory, i.e. we can neglect all terms of $O\left(\varepsilon^{2}\right)$, we can write:

$$
\begin{aligned}
& P_{c}=P_{c}{ }^{(0)}+\varepsilon P_{c}^{(1)}+O\left(\varepsilon^{2}\right) \\
& \Theta_{c}=\Theta_{c}{ }^{(0)}+\varepsilon \Theta_{c}{ }^{(1)}+O\left(\varepsilon^{2}\right)
\end{aligned}
$$

Upon substitution of (4.35) into (4.34) with the definition (4.33) a sequence of problems emerges as equal powers of $\varepsilon$ are collected.

At $O(1)$ the unperturbed Kirchhoff problem is obviously recovered so that:

$$
P_{c}{ }^{(0)}=\rho_{c} \quad \text { and } \quad \Theta_{c}^{(0)}=\vartheta_{c}
$$

where $\rho_{c}$ is given by $(4.18)$ and $\vartheta_{c}=0$ or $\pi$. At $O(\varepsilon)$ we obtain the linear correction to the position of the critical points which, after some algebra, is found to be:

$$
\begin{aligned}
& \mathrm{P}_{c}{ }^{(1)}=\frac{\operatorname{coshm} \rho_{c} \cos m \vartheta_{c} e^{-m \rho_{c}}}{2\left[\Lambda \cosh 2 \rho c-e^{\left.-2 \rho_{c}\right]}\right.}\left(e^{s t}+e^{-s t}\right) \\
& \Theta_{c}{ }^{(1)}=\frac{\chi \sinh m \rho c \cos m \vartheta_{c} e^{-m \rho_{c}}}{2\left[\Lambda-e^{-2 \rho_{c}}\right]}\left(e^{s t}-e^{-s t}\right)
\end{aligned}
$$

where $\Lambda \equiv(1-\lambda) /(1+\lambda)$. The equations (4.37) describe the trajectory of the crtical point in the corotating frame according to linear perturbation theory. Let us first consider the displacement in the location of the critical points at $t=0$ due to the perturbation.

It is easily seen from $(4.37 \mathrm{~b})$ that $\Theta_{c}{ }^{(1)}(t=0)=0$. This is simply due to our choice $(4.30)$ to perturb the ellipse with the $\operatorname{cosm} \vartheta$ mode alone. The more interesting fact is that, without too much effort, one can show that, for the case

measuring the rate of change of the inclination of the major axis of the fitted ellipse to the $\mathrm{x}$-axis differs from $\Omega_{\mathrm{k}}$ by amounts of the order of less than $1 \%$ for times smaller than $t_{f}$. 
$m=3 \dagger$, for the critical point $S_{1}$ (cf. Figure 4.2) - i.e. the one at $\vartheta_{c}=0-\Theta_{c}{ }^{(1)}<0$ for $t>0$ so that $S_{1}$ is initially moved into the $y<0$ half-plane. This indeed corresponds to the side of the vortex where the filament is extracted (cf Figure 4.1), and can be considered as the first prediction of this linear theory.

Even more surprising is the investigation of (4.37a). With a bit of ingenuity it can be shown that the denominator in (4.37a) is always positive so that the sign of $P_{c}{ }^{(1)}(t)$ is the same as the sign of $\operatorname{cosm} \vartheta_{c}$. For a mode 3 perturbation this means that that $S_{1}$ is actually "pushed away" as the perturbation grows, while $S_{2}$ approaches the origin. This remarkable fact is indeed supported by the numerical calculation that we are going to present next.

Contrary to what one might have guessed, the scenario that leads to the filamentation of an ellipse perturbed with an unstable normal mode can be summarized as follows: as the perturbation grows $S_{1}$ is pushed further away from the origin but the vortex boundary grows outwards faster than the critical point recedes and, after overtaking, a filament forms. At the non-filamenting end, on the contrary, $S_{2}$ actually gets closer to the origin, but the receding vortex boundary contracts fast enough that the distance between $S_{2}$ and the contour always remains $O(1)$.

Before presenting the numerical simulations, we carry out the last step of the linear analysis that leads to the estimation of the filamentation time $t_{f}$. From (4.20), (4.22) and (4.31) it is easy to obtain the equation that describes the evolution of the vortex boundary, which is found to be

$$
\rho_{v}(\vartheta, t)=\rho_{0}+(1 / 2) \varepsilon \frac{\left(e^{s t}+e^{-s t}\right) \cos m \vartheta+\chi\left(e^{s t}-e^{-s t}\right) \sin m \vartheta}{\lambda \cos 2 \vartheta+(1 / \lambda) \sin 2 \vartheta}
$$

When the critical points coincides with the perturbed vortex boundary, its coordinates given by (4.37), must also be solutions of (4.38). Therefore $t_{f}$ is found by substituting (4.37) into (4.38) and solving for the time. After some algebra the following result is obtained:

$$
t_{f} \approx \frac{1}{s} \log \left(\frac{\rho_{c}-\rho 0}{\varepsilon \Xi(\lambda)}\right)
$$

I It may be useful, for the sake of clatiry, to remind the reader that although a mode $\mathrm{m}$ on a circular vortex gives rise to $\mathrm{m}$ critical points, a perturbed ellipse possesses only two critical points for any mode $\mathrm{m}$ (as shown in Figure 4.2) provided its amplitude is "small". 
where $\Xi$ is a geometric factor that depends on the aspect ratio $\lambda$ of the unperturbed ellipse and given by:

$$
\Xi(\lambda)=\frac{1}{2 \lambda}-\frac{\cosh m \rho_{c} e^{-2 \rho_{c}}}{2\left[\Lambda \cosh 2 \rho_{c}-e^{-2 \rho_{c}}\right]}
$$

with $\rho_{c}$ given by (4.18). The reason for the $\approx$ sign in (4.39) is twofold. In the first place, to obtain (4.39) the orders of the asymptotic perturbation theory have had to be mixed (notice the $\varepsilon$ appearing in the argument of the logarithm). Secondly, the terms proportional to $e^{-s t}$ have been neglected in comparison with the ones proportional to $e^{\text {st }}$, which approximation can be validated after the calculation of $t_{f}$ from (4.39) (one could, of course, keep the $e^{-s t}$ terms and derive an expression for $t_{f}$ by solving a quadratic).

In simpler terms, (4.39) is obtained by equating the radius of the vortex given by $\rho_{0}+\varepsilon \rho_{1} e^{\text {st }}$ with the radius of the critical point given by $\rho_{c}+\varepsilon \rho_{2} e^{\text {st }}$, where $\rho_{1}$ and $\rho_{2}$ are constants, and solving for t. Provided $\varepsilon$ is small, the $O\left(\varepsilon^{2}\right)$ terms can be neglected without too much ado. Of course $\rho_{c}-\rho_{0}$ also ought to be small if it has to be balanced by small terms proportional to $\varepsilon$, which, as can be seen from Figure 4.3 , turns out to be the case. Thus, for instance, for a $\lambda=0.3$ ellipse $\rho_{c}-\rho_{0} \approx 0.19$ and for $\lambda=0.2 \rho_{c}-\rho_{0} \approx 0.14$. We believe that it is the closeness of the critical points of the unperturbed ellipse to the boundary that makes (4.39) a better predictor than one might have expected.

Notice that the factors entering (4.39) appear in the intuitively correct position. Thus $t_{f}$ is inversely proportional to the growth rate $s$, so that more unstable disturbances will tend to filament sooner. Also (4.39) shows that less elongated ellipses (i.e. larger $\rho_{c}-\rho_{0}$ ) and smaller perturbation amplitudes will retard filamentation.

Armed with (4.39) we now present some of the numerical experiments that we have performed to test the extent to which the above linear analysis can applied to the fully nonlinear evolution of a perturbed Kirchhoff vortex. In all of these runs the errors in the globally conserved quantities we have measured (area and first moments) are of the order one part in $10^{-4}$. The algorithm we have used is the same as the one described in Section 1.5.2.

The initial condition for these runs are ellipses perturbed according to (4.20) together with (4.30). Thus an initial condition is entirely specified by the choice of the aspect ratio $\lambda$ of the ellipse together with the mode $m$ and amplitude $\varepsilon$ of the 
perturbation. We start by showing the evolution of a slightly unstable $\lambda=0.30^{\dagger}$ ellipse with a mode 3 perturbation of amplitude $\varepsilon=0.02$ in Figure 4.4. The crosses are drawn at the location of the critcal points of the instantaneous corotating streamfunction (the technique used for calculating their position is presented in Appendix D; we simply note here that it loses its validity when the vorticity patch becomes very different from an ellipse, as is the case after the filament is extracted).

Notice how after approximately $3 / 4$ of a full rotation (for $\lambda=0.3$ the period $\mathrm{T}_{\mathrm{k}}=2 \pi / \Omega_{\mathrm{k}} \approx 35.4$ ) the critical point $S_{1}$ originally to the right of the ellipse comes in contact with the contour and almost immediately thereafter the filament is seen to emerge. To quantify the filamentation time we plot in Figure 4.5 the time evolution of the distance of the critical point from the origin $d_{c p}$ and the quantity $d_{\max }$ the location of the "nose" of the ellipsef. As was shown with the linear theory, the critical point recedes from the origin but the growth of the perturbation is such that the boundary eventually overtakes it.

The time $t_{c}$ at which $d_{c p}$ and $d_{\max }$ cross is found to be approximately $t_{c} \approx 28.5$. This compares quite well which the prediction of linear theory from (4.39) which gives $t_{f} \approx 30.4$. Notice also, from Figure 4.4 how the distance between the critical point $S_{2}$ and the boundary of the vortex actually increases due to the receding contour.

A clear diagnostic that easily quantifies the emergence of the filament is the minimum value of the curvature along the contour plotted versus time, shown in Figure 4.5a. Recall that for an ellipse (and even for a perturbed one, as long as the perturbations is small) the curvature is positive for every point on the contour. The appearence of negative curvature is a sure sign that a filament has been created (cf. Figure 4.4). It is a simple matter to show that the minimum value of the curvature on an exact ellipse is equal to $\lambda^{3 / 2}$, which for $\lambda=0.3$ is approximately 0.16 . The value of $\min (\kappa)$ is not far from this number as the perturbation grows, but can be seen to become very large and negative as the filament emerges.

As a second example we show in Figure 4.6 the evolution of a more elongated $\lambda=0.2$ ellipse with an $m=4$ perturbation of amplitude $\varepsilon=0.02$. Because the initial vorticity field is symmetric upon reflection about the origin, both critical points

† Recall that the stability boundary for the Kirchhoff ellipse is $\lambda=0.333 \ldots$ below which mode 3 becomes unstable. $F_{d_{\text {max }}}$ is defined as the maximum distance of the contour from the origin. That this corresponds to the nose of the ellipse is confirmed by the fact that the plot of the distance from the origin to the point of maximum curvature around the contour was found to be indistinguishable from the curve for $\mathrm{d}_{\max }$. 
actually come into contact with the contour, and two filaments are extracted, as could have been seen from ( $4.37 a$ and $b$ ). The curves for $d_{c p}$ and $d_{\max }$ are shown in Figure 4.7, and the crossing time is found to be $t_{c} \approx 14.3$. Again the prediction of linear theory $t_{f} \approx 17.5$ is found to be quite good. In Figure $4.7 \mathrm{a}$ the minimum value of the curvature is seen to become large and negative as the filament makes its appearance.

In conclusion we show, as a counter example, the evolution of a $\lambda=0.25$ ellipse perturbed with a stable $m=4 \dagger$ mode of amplitude $\varepsilon=0.02$. Note that the evolution shown in Figure 4.8 is actually fully nonlinear, but the nonlinear interactions cannot generate any $m=3$ component, which is the only linearly unstable mode. We have integrated numerically up to $t \approx 3 T_{k}$ without observing any sign of filamentation, which is consonant with the fact that the critical points are. always outside the boundary of the vortex.

In Figure 4.9 plot the evolution of $d_{c p}$ and $d_{\max }$. Note how the critical points move in phase with the pulsations of the perturbed vortex, approaching the origin as the vortex contracts and receding as the vortex elongates. That these curves are not simple periodic functions is not too surprising because the evolution they are describing is actually nonlinear. The crucial point, however, is that as long as the critical points are outside the voticity boundary no filamentation is observed. We remark, incidentally, that the actual distance between the critical point and the contour is in fact quite small ( $\min \left[d_{c p}-d_{m a x}\right] \approx 0.08$ for a major axis $a=2.0$ ).

We believe that the evidence presented here incontrovertibly establishes the connection between the process of filamentation and the presence of critical (hyperbolic) points in the comoving streamfunction that come into contact with the boundary. We are now going to extend this idea from the Euler equations to the equivalent barotropic model, and show that the discovery of suppressed filamentation at large $\gamma$ reported in section 2.3.3 can analogously be understood from the absence of critical points near the boundary of the vortices.

$\dagger_{\text {It }}$ is easy to show from (4.25) that the mode $m=4$ loses stability below $\lambda \approx 0.216$. 


\subsection{Critical Points in the Equivalent Barotropic Model $(\gamma>>1)$}

As for the Euler case, it will prove useful to start by analyzing the case of a perturbed circualr vortex before turning to more complicated vorticity distributions. We consider an equivalent barotropic circular vortex of radius $R$ and constant potential vorticity $\Pi=\left(\nabla^{2}-\gamma^{2}\right) \psi=\Pi_{0}$, surrounded by fluid with $\Pi=0$. Without loss of generality we choose $\Pi_{0}=1$. The streamfunction that satisfies:

$$
\begin{array}{lll}
\left(\nabla^{2}-\gamma^{2}\right) \psi_{i}=1 & \text { for } & r<R \\
\left(\nabla^{2}-\gamma^{2}\right) \psi_{0}=0 & \text { for } & r>R
\end{array}
$$

together with continuity of pressure and velocity (i.e. $\psi$ and $\psi_{r}$ ) at the boundary is easily found to bet :

$$
\begin{array}{llr}
\psi_{i}=R \gamma^{-1} K_{1}(\gamma R) I_{0}(\gamma r)-\gamma^{-2} & \text { for } & r<R \\
\psi_{0}=-R \gamma^{-1} I_{1}(\gamma R) K_{0}(\gamma r) & \text { for } & r R
\end{array}
$$

where $I_{m}$ and $K_{m}$ are modified Bessel functions of order $m$. We now consider infinitesimal perturbations on this vortex proportional to $e^{i(m \vartheta-\omega t)}$ and wish to find the dispersion relation for such waves on the circular boundary. Among the many ways to solve this problem, we illustrate the one used by Pedlosky (1985) for the heton cloud problem. Let $\varphi$ designate the perturbation streamfunction. It must satisfy the homogeneous version of equation (4.41) and be continuous at the boundary, and is easily shown to be given by:

$$
\begin{array}{lll}
\varphi_{i}=\varepsilon I_{m}(\gamma r) / I_{m}(\gamma R) e^{i(m \vartheta-\omega t)}+c c . & \text { for } & r<R \\
\varphi_{o}=\varepsilon K_{m}(\gamma r) / K_{m}(\gamma R) e^{i(m \vartheta-\omega t)}+c c . & \text { for } & r<R
\end{array}
$$

where $\varepsilon$ is an infinitesimal. The last matching condition needed is readily obtained by integrating the equation of motion:

$$
\left[\partial_{t}+J(\Psi, \cdot)\right] \Pi=0
$$

I In this section we revert to the use of $r$ and $\vartheta$ as circular polar cordinates. 
with $\Psi \equiv \psi+\varphi$ across the boundary of the vortex. From the $O(\varepsilon)$ terms it is found that the quantity that ought to be made continuous at the boundary is:

$$
\left(\partial_{t}+(V / r) \partial_{\vartheta}\right) \partial_{r} \psi-r^{-2}\left(\partial_{\vartheta} \psi\right) \partial_{r}(r V)
$$

where $V \equiv \partial_{r} \psi$ is the azimuthal velocity of the unperturbed vortex. With (4.42) and (4.43), some simple algebra yields the dispersion relation for waves on the boundary of an equivalent barotropic vortex:

$$
\omega(m)=m\left[I_{1}(\gamma R) K_{1}(\gamma R)-I_{m}(\gamma R) K_{m}(\gamma R)\right]
$$

Note that the only nondimensional parameter of interest in this problem is $\gamma \mathrm{R}$ the ratio of the radius of the vortex to the deformation radius. Using the property (cf., for instance Abramowitz and Stegun 1964):

$$
\lim _{z \rightarrow 0} I_{v}(z) K_{v}(z)=\frac{\Gamma(v)}{2 \Gamma(v+1)}=\frac{1}{2 v}
$$

is easily follows that $\omega=1 / 2$ for $m=2$, so that the Euler result (4.2) is recovered in the $\gamma \rightarrow 0$ limit. The more interesting behavior in the equivalent barotropic model is however observed at $\gamma>>1$, where the property

$$
I_{v}(z) K_{v}(z) \approx \frac{1}{2 z}\left(1-\frac{1}{2} \frac{4 v^{2}-1}{8 z^{2}}\right) \quad \text { for } \quad z>>1
$$

can be used to show that:

$$
\omega \sim \frac{m\left(m^{2}-1\right)}{8}\left(\frac{1}{\gamma R}\right)^{3}
$$

As we have mentioned in Chapter 2, a time scale proportional to $\gamma^{3}$ appears from a number of different problems for the equivalent barotropic model at $\gamma>>1$.

From the dispersion relation it is a simple matter to determine the location $r_{c}$ of the critical circle for mode $\mathrm{m}$ - which for $\varepsilon \neq 0$ opens into $\mathrm{m}$ ghost vortices with $\mathrm{m}$ critical points located at $r_{c}+O(\varepsilon)$. Its position is given by equating $V_{0}=\partial_{r} \psi_{0}$ with the quantity $\Omega r$, where $\Omega \equiv \omega / \mathrm{m}$. The equation to be solved for $r_{c}$ then becomes:

$$
I_{1}(\gamma R) K_{1}\left(\gamma r_{c}\right)=\left[I_{1}(\gamma R) K_{1}(\gamma R)-I_{m}(\gamma R) K_{m}(\gamma R)\right] r_{c}
$$


Before showing the numerical solution of (4.45) it is useful to derive the asymptotic expressions. Let us concentrate on the elliptical mode $m=2$. The quantity of interest is $r_{c} / R$ as a function of $\gamma R$. The $\gamma \rightarrow 0$ limit is quite obviously identical to the Euler result given in (4.6):

$$
\lim _{z \rightarrow 0} \frac{r c}{R}=\sqrt{ } 2
$$

The $p>>1$ limit is, however, not as simple to deduce. Notice first that, for $m=2$,

$$
\Omega \approx(3 / 8)(\gamma R)^{-3} \quad \text { for } \quad \gamma>>1
$$

This might suggest that, since $\Omega \rightarrow 0$ as $\gamma \rightarrow \infty$, the critical points should move to infinity in that limit. This however turns out not to be the case, and the reason is to be found in the fact that the velocity field vanishes exponentially away from the vortex. Substituting the ansatz $r_{c}=R+\delta-$ with $\delta<<1-$ into (4.45) and using the asymptotic forms for the modified Bessel functions leads to the result:

$$
\delta \approx 2 \frac{\log \gamma}{\gamma} \text { for } \gg>1
$$

Thus, perhaps surprisingly, as $\gamma \rightarrow \infty$ the critical points of the $m=2$ perturbation become coincident with the vortex boundary. Notice, however, that their approach to the boundary is a very weak function of $\gamma$.

The numerical solution of (4.45) shows that there is an even richer behavior as $\gamma$ is varied from zero to infinity. It is shown in Figure 4.10 , obtained using a very simple bisection method (see, for instance, Press et al. 1986). It turns out that as $\gamma$ is made finite the critical points at first recede from the vortex, reaching a maximum distance around $\gamma \approx 5$, but at even larger $\gamma$ they approach the vortex with a very slow $\log \gamma / \gamma$ decay. In practical terms, therefore, the $\log \gamma / \gamma$ behavior means that, even at very large $\gamma$ the critical points are "far" from the vortex (cf. Figure 4.10).

This is, we believe, the reason for the absence of filamentation at $r>>1$ that was observed in Chapter 2. To confirm this we present a numerical initial value calculation at $\gamma=3$. The condition at $t=0$ was chosen to be an exact $\lambda=0.3$ ellipse. Since the ellipse is not a solution of the equivalent barotropic equation, although, as we have shown in Chapter 2 , it is not far from an equivalent barotropic rotating $V$-state, it will not maintain its shape for $t>0$.

The evolution is shown in Figure 4.11, where the computation has been carried out to two full revolutions of the vortex $\left(t_{\max }=1000\right)$. The need for going so 
far in time is dictated by the $\gamma^{3}$ time scale present. Since, as was shown in the previous section, an Euler ellipse filaments on times of $\mathrm{O}(10)^{\dagger}$, for large $\gamma$ the calculation should be extended to times $O(100-1000)$ before a conclusive statement regarding filamentation can be made. Compare the rotation period of an Euler $\lambda=0.3$ ellipse, $T_{k} \approx 35$, with the time required for a full rotation of the vortex at $\gamma=3$, which, as can be deduced from Figure 4.11 is close to 500 .

Notice how the critical points remain at an $O(1)$ distance throughout the evolution. In Figure 4.12 we have plotted their distance from the origin $-d_{c p}-$ as a function of time, together with the maximum distance from the origin to the vortex boundary $-d_{\max }$. As was observed for the Euler case the quantities are in phase, as the critical point approaches and retracts from the vortex while the latter undergoes large amplitude pulsations. There is, however, no apparent sign of a trend that would indicate a long time approach of the critical point to the contour.

In summary, the results presented in this chapter leave little doubt as to the the direct connection between the formation of vorticity filaments and the presence of critical (hyperbolic) points of the comoving steamfunction. It is our hope and belief that a geometric approach to the understanding of the fully nonlinear dynamics of coherent vortex structures - such as the one we have illustrated here, will prove very useful in shedding light on a number of other phenomena that have, until now, escaped simple explanations.

† Recall that these are nondimensional units, and that time is scaled by the constant value of the geostrophic potential vorticity inside the ellipse. 


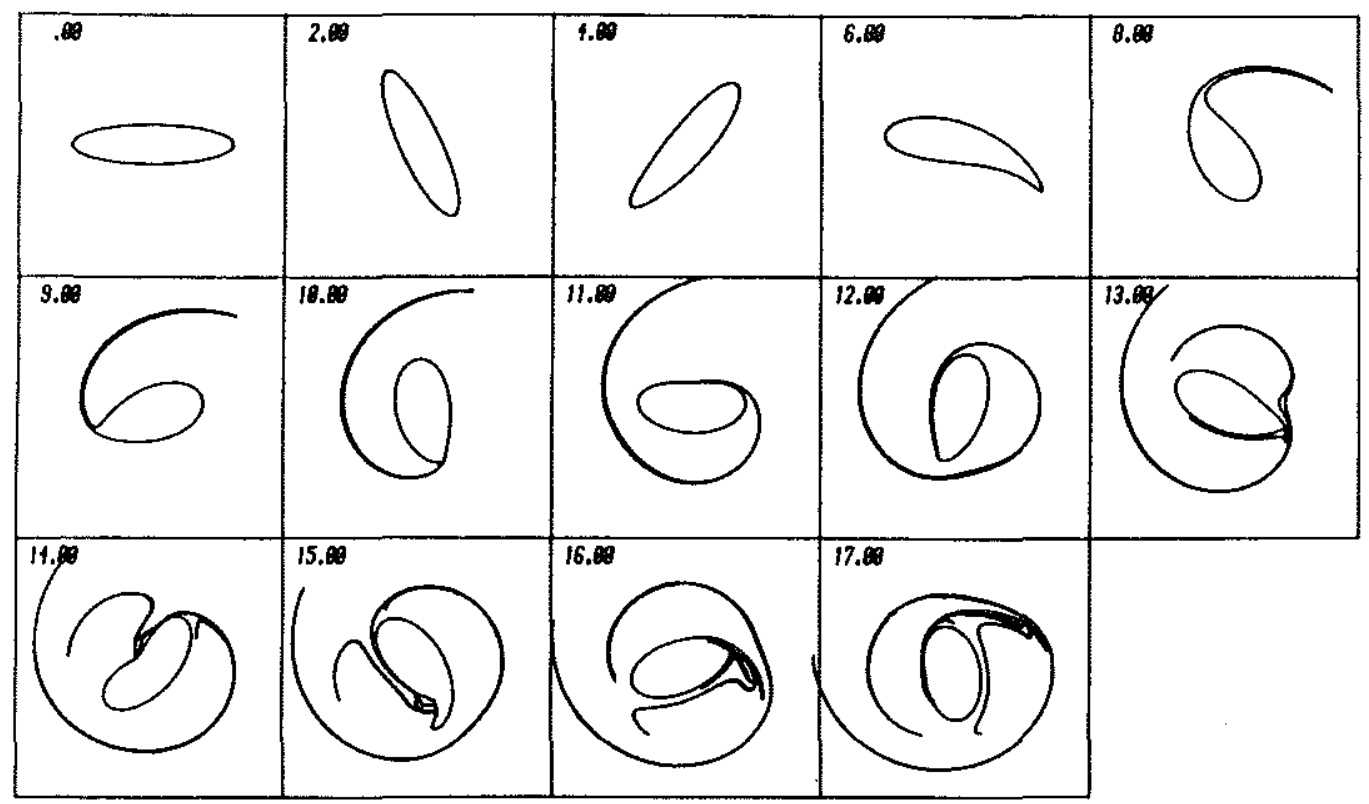

FIGURE 4.1. The evolution of a 1:4 Kirchhoff ellipse with an inital normal mode 3 perturbation of amplitude $\varepsilon=0.005$. (Reprinted from Dritschel, 1988). The vorticity is constant within the ellipse and its value is $2 \pi$. 


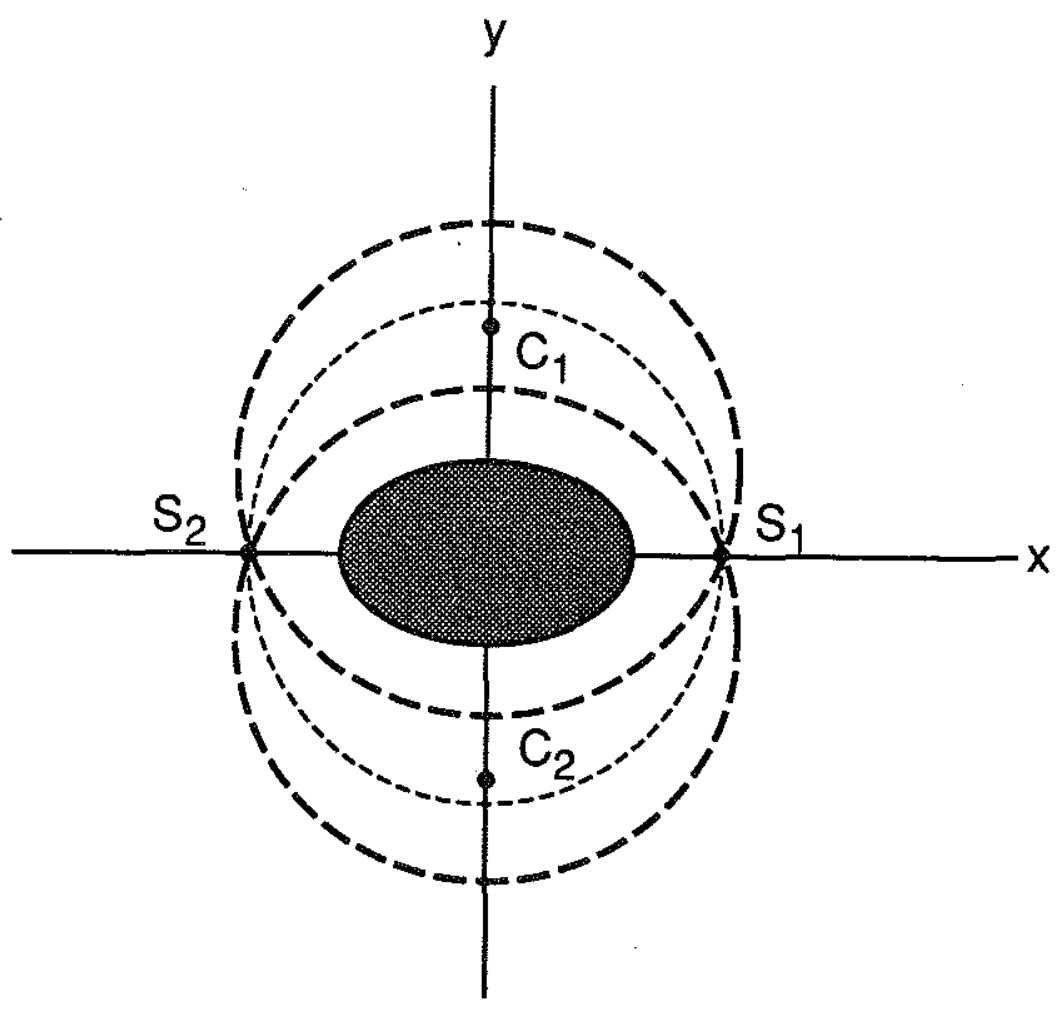

FIGURE 4.2. A sketch of the geometry of the corotating streamfunction for a circular vortex with an $m=2$ (elliptical) perturbation. The solid line is the unperturbed circle. The thin dashed line is the critical circle. The dark dashed lines are the separatrices. $C_{1}$ and $C_{2}$ are the centers of the ghost vortices, and $S_{1}$ and $S_{2}$ are the hyperbolic critical ponts. 


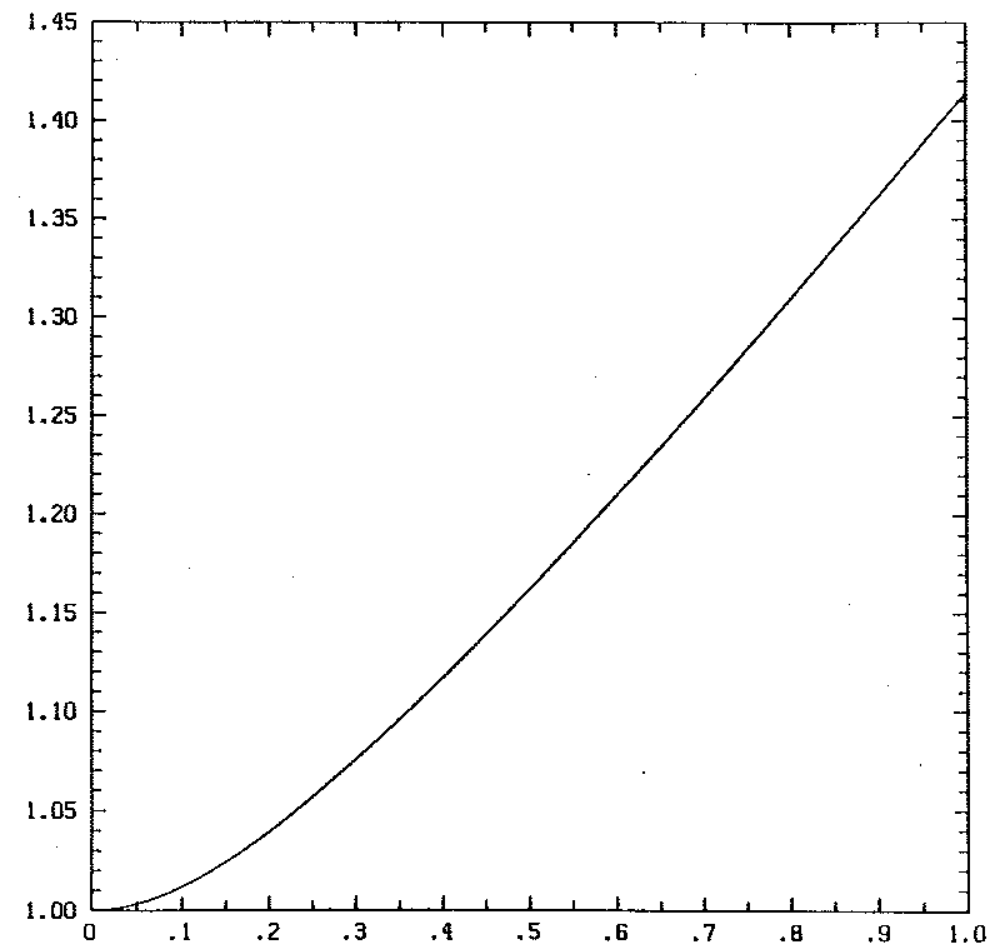

FIIGURE 4.3. The ratio of the distance of the critical point to the origin to the major axis of the Kirchhoff ellipse ( $x_{d} / a$ in the text) vs. the aspect ratio $\lambda$. 


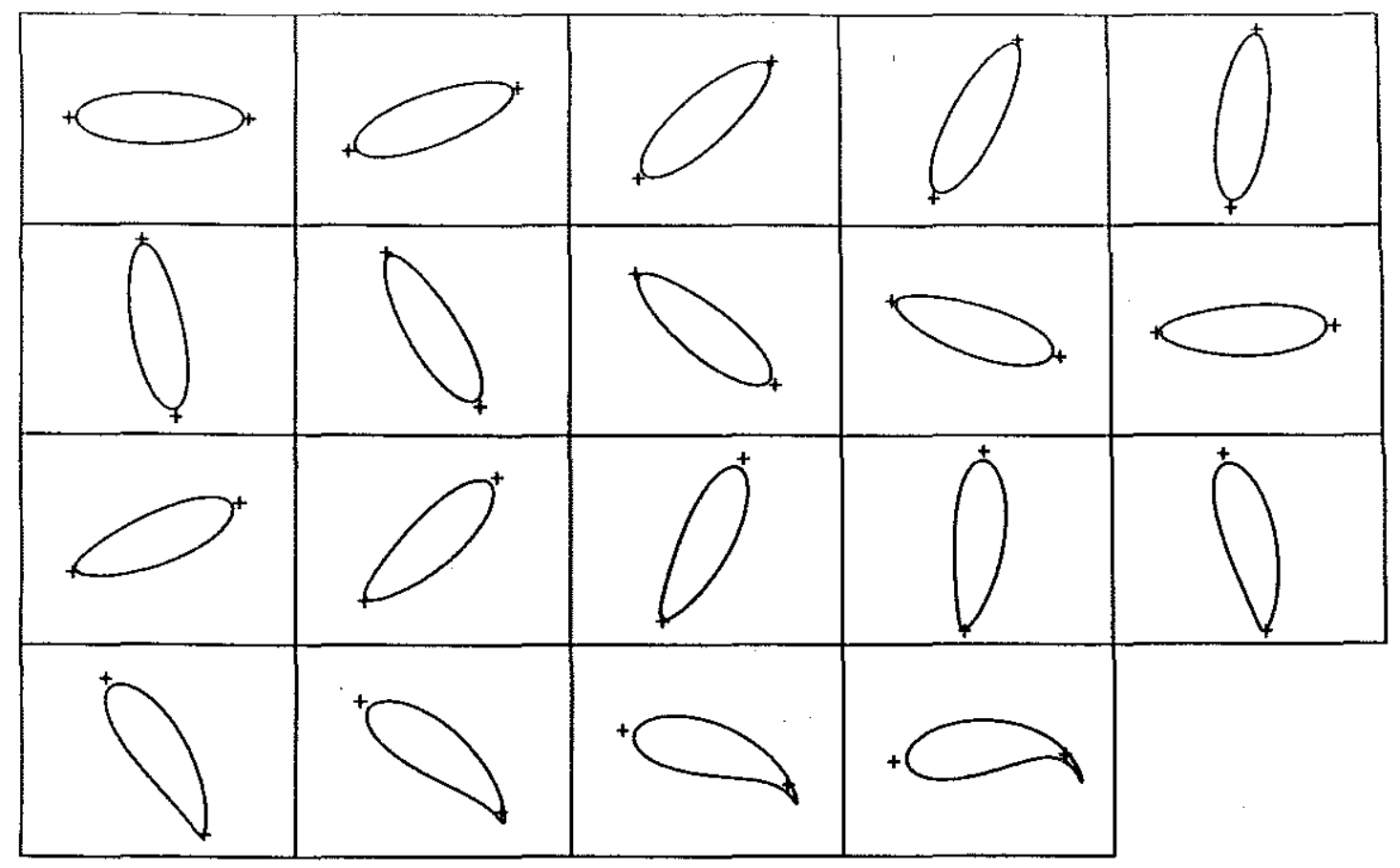

FIGURE 4.4. The evolution of a $\lambda=0.30$ Kirchhoff ellipse, initially perturbed with an unstable $m=3$ Love mode of amplitude $\varepsilon=0.02$. The crosses designate the locations of the hyperbolic critical points of the instantaneous corotating streamfunction obtained by the method of Appendix D. Times advances to the left and downwards, and the frames shown correspond to $t=0,2,4,6$, etc. 


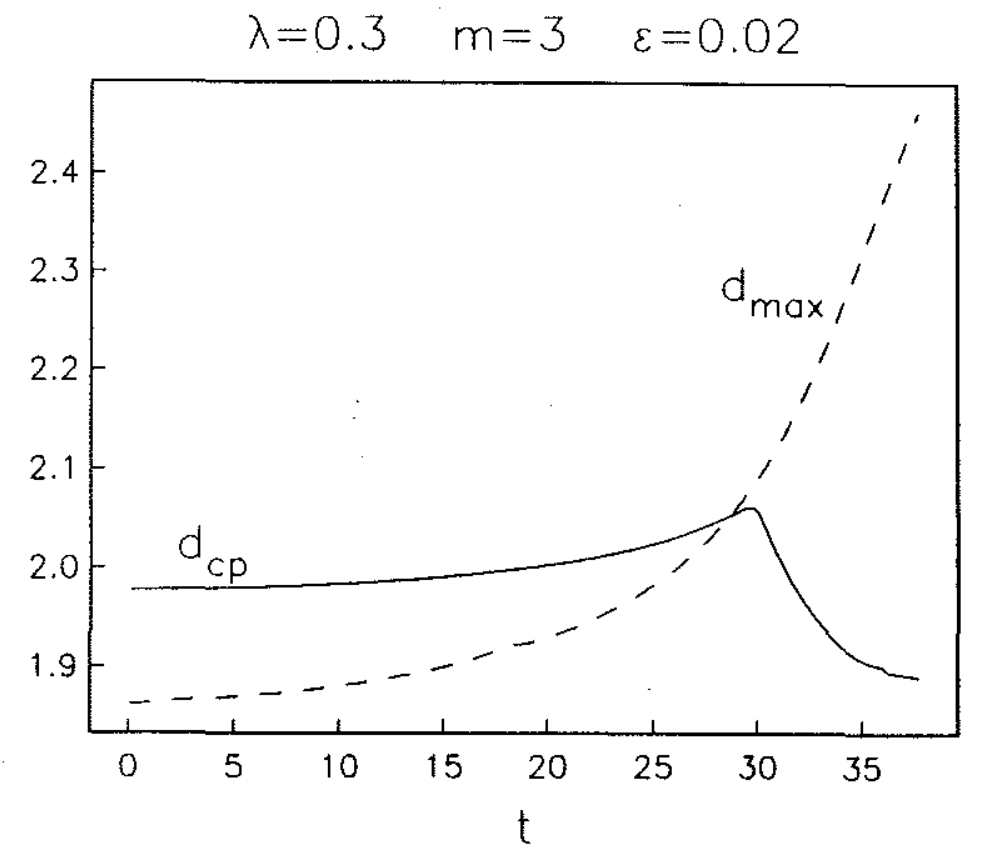

FIGURE 4.5. Plots of the maximum distance of the contour from the origin ( $d_{\text {max }}$ ) and of the distance from the critical point to the origin $\left(d_{c p}\right)$ as a function of time for the evolution shown in Figure 4.4. The crossing occurs at $t \approx 28.5$ when filamentation is observed. 


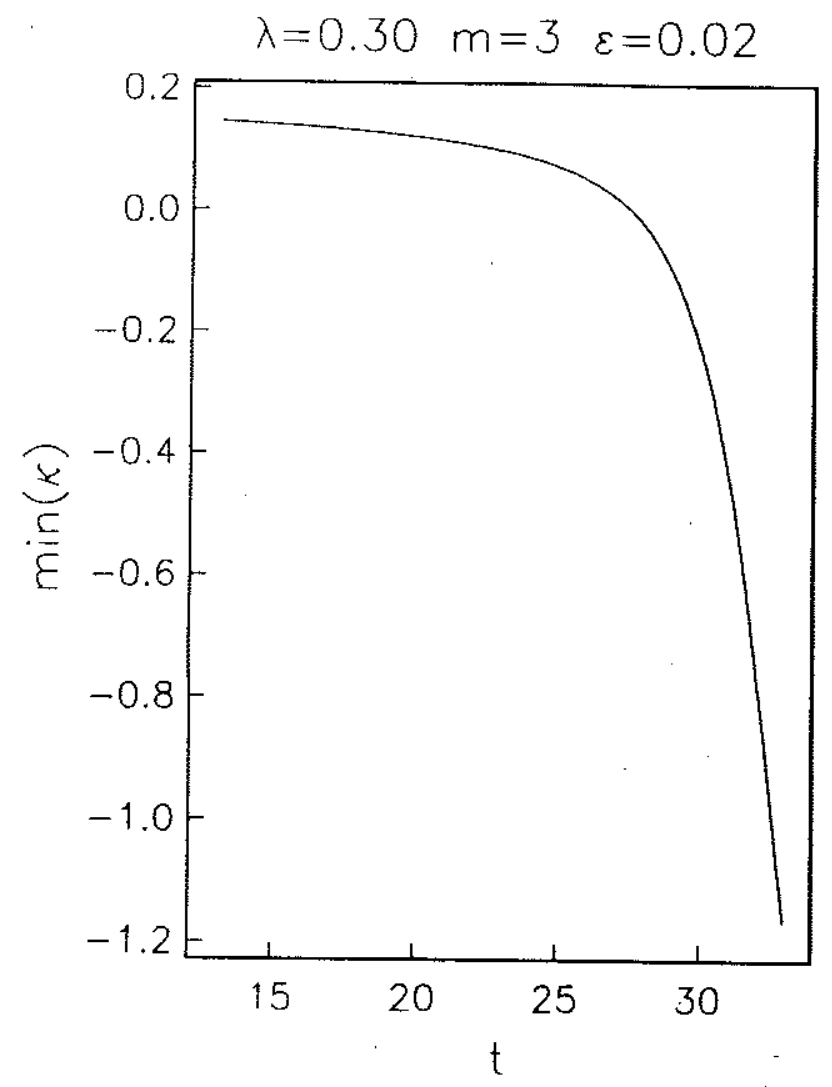

FIGURE 4.5a. The evolution of the minimum value of the curvature along the contour vs. time for the perturbed ellipse of Figure 4.4. Note the appearance of large negative curvatures near $t_{c}$ signalling the formation of a filament. 


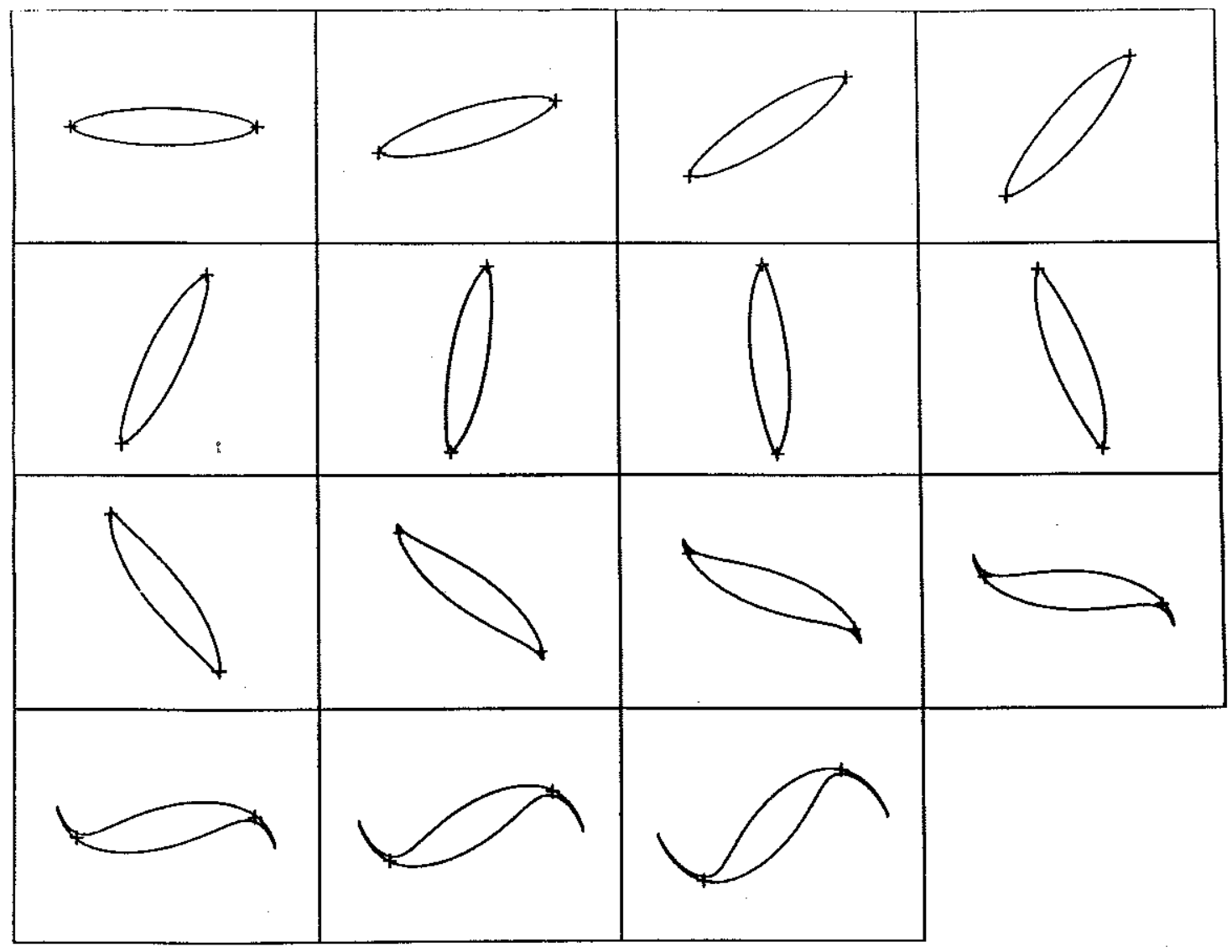

FIGURE 4.6. Evolution of a $\lambda=0.20$ ellipse with an initial $m=4, \varepsilon=0.02$ perturbation: The time frames shown are $t=0,2,4,6$, etc. 


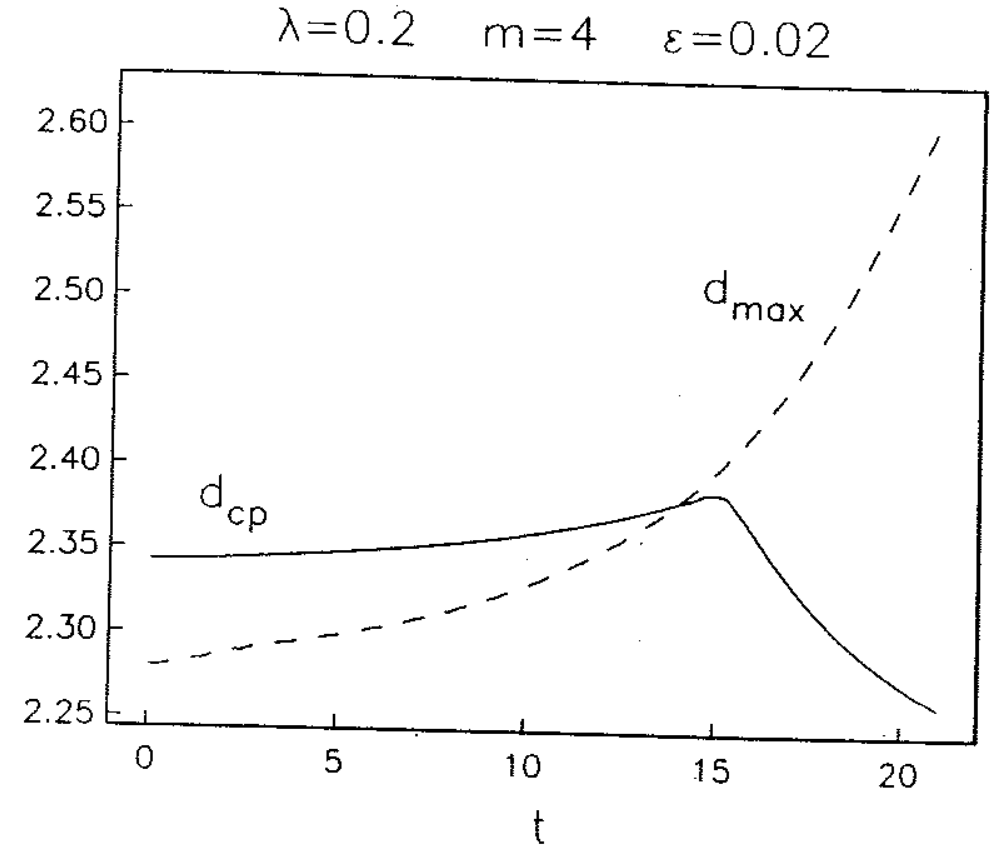

FIGURE 4.7. Same as Figure 4.5 but for $\lambda=0.20, m=4$ and $\varepsilon=0.02$. Here $t_{c} \approx 14.3$. 


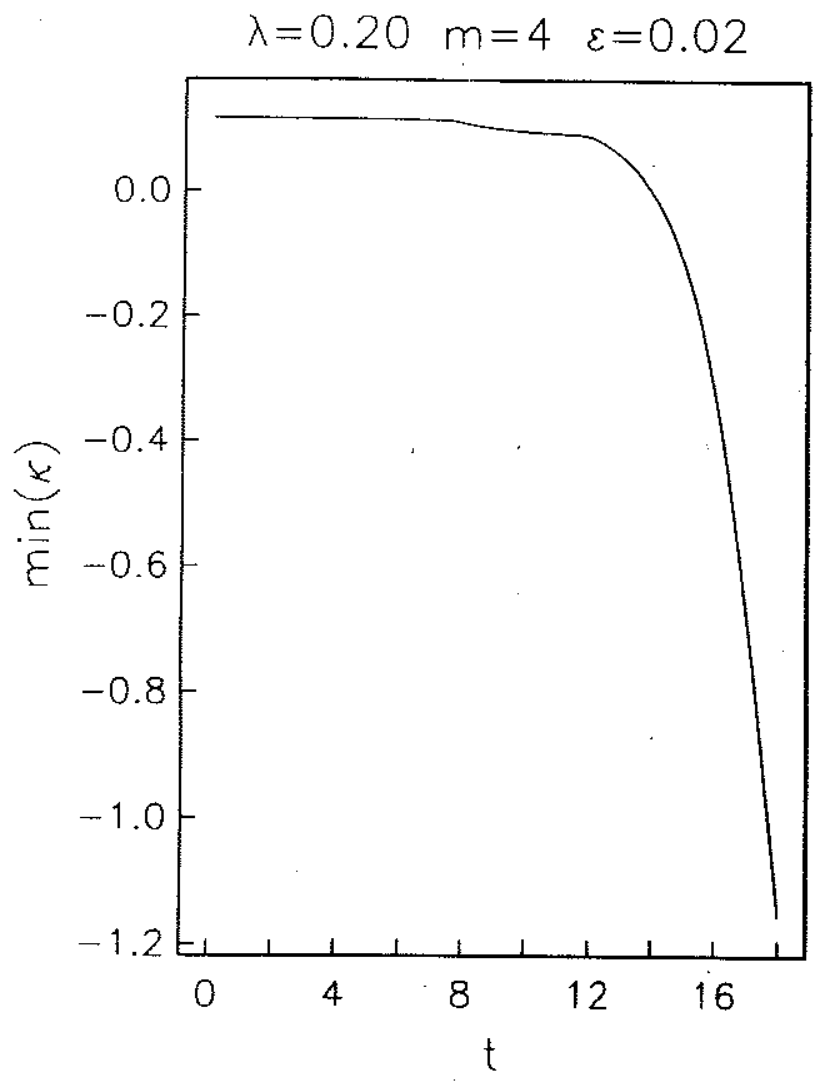

FIGURE 4.7a. Same as Figure 4.5a but for $\lambda=0.20, m=4$ and $\varepsilon=0.02$. Here $t_{c}=14.3$. 


\begin{tabular}{|l|l|l|l|l|}
\hline & 0 & & & \\
\\
\hline
\end{tabular}

FIGURE 4.8. The evolution of a $\lambda=0.25$ ellipse with an initial stable Love perturbation of mode 4 and amplitude $\varepsilon=0.02$. Note that the critical points never penetrate the contour, and no filamentation is observed. The times shown are $t=0,4,8,12$, etc. 


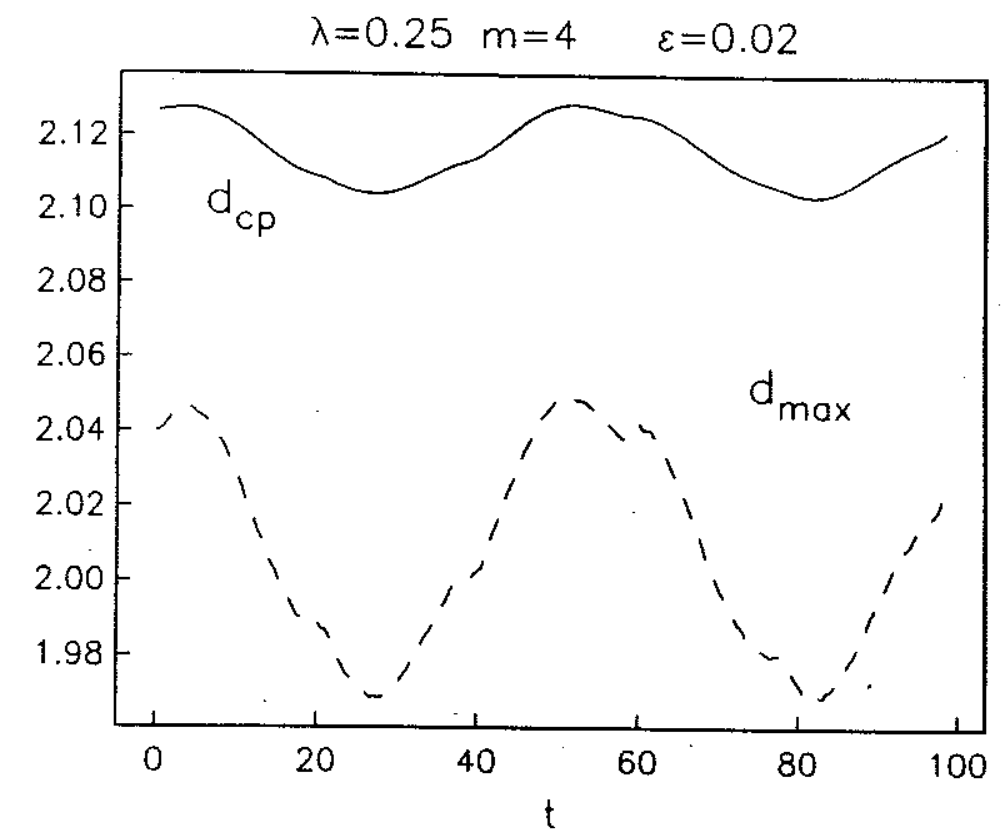

FIGURE 4.9. Same as Figure 4.5 but for the stable $m=4$ evolution of Figure 4.8 . 


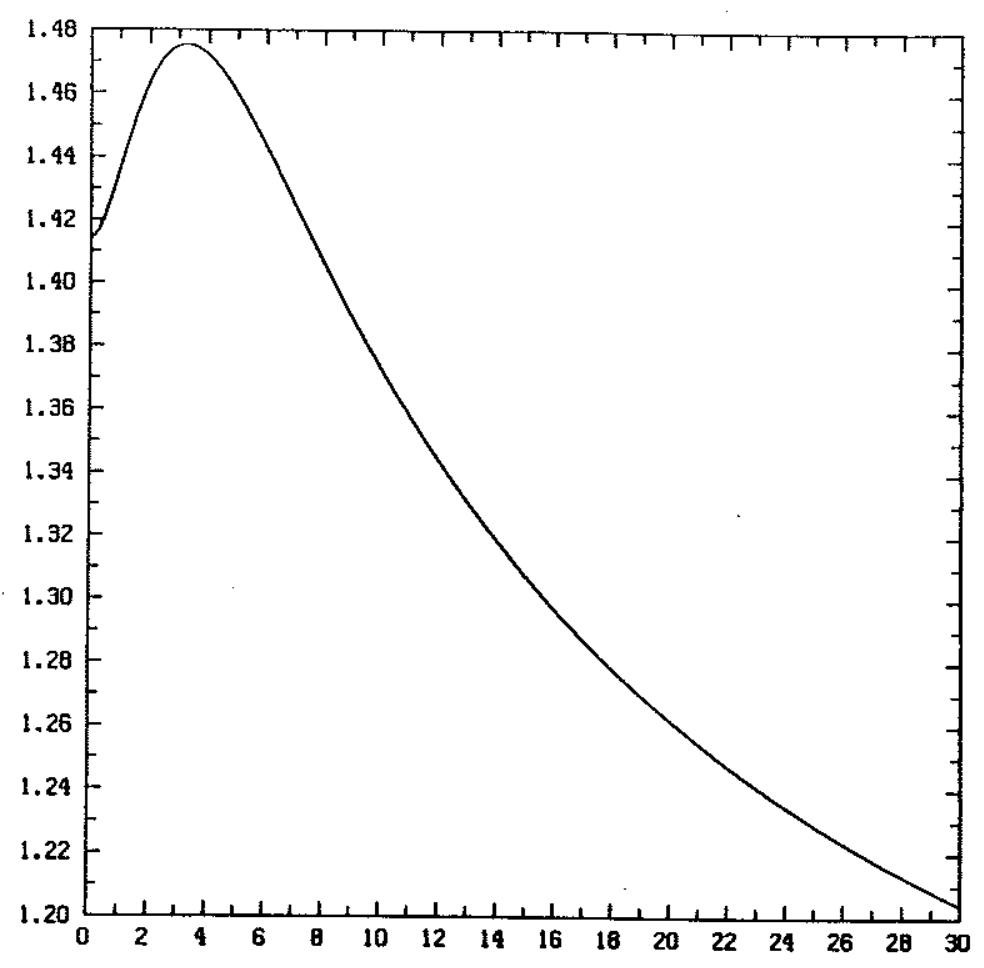

FIGURE 4.10. The the critical radius as a function of $\gamma$ for a circular equivalent barotropic vortex of radius 1 . The maximum distance occurs around $\gamma \approx 3.3$. 


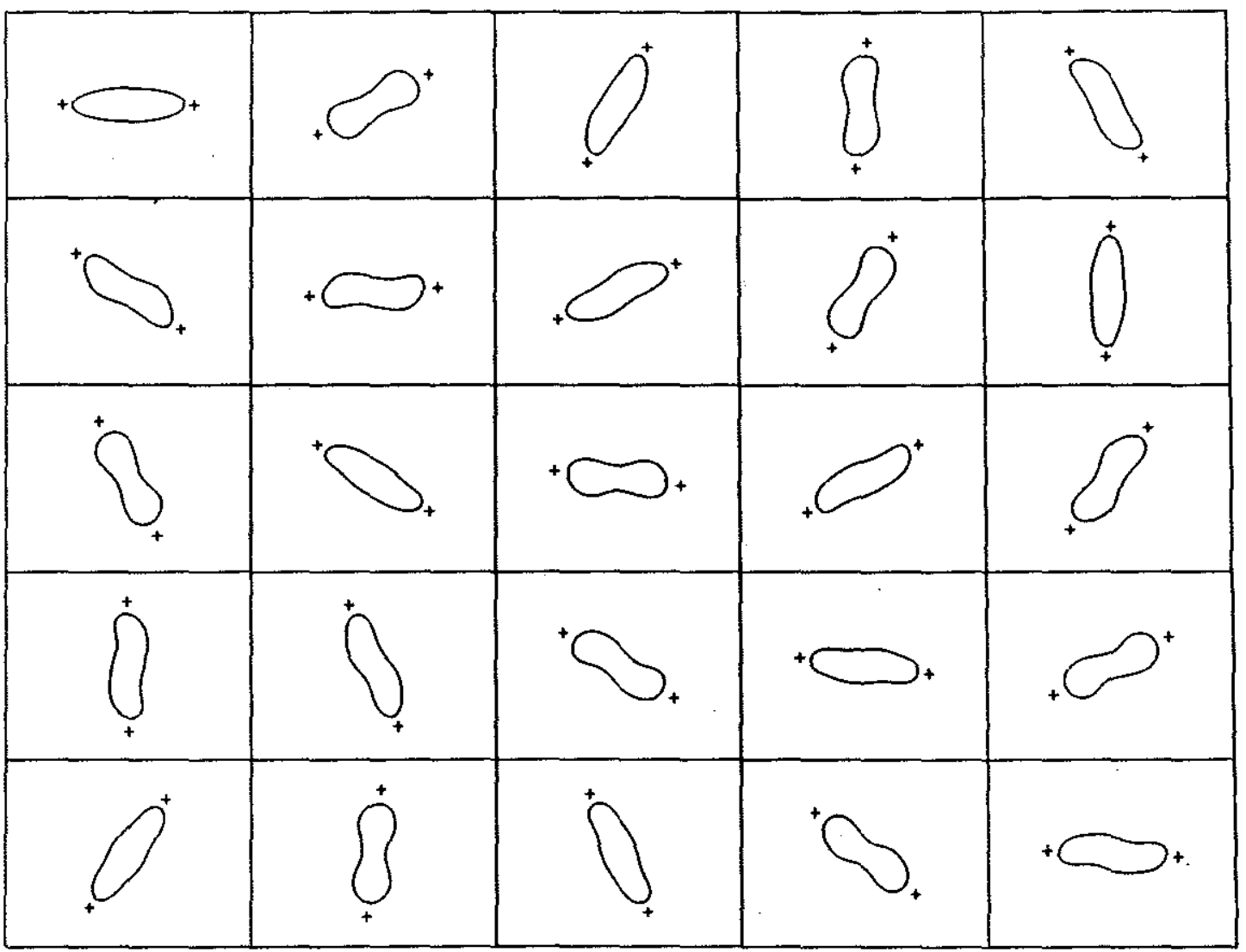

FIGURE 4.11. The evolution of an initially elliptical $\lambda=0.30$ equivalent barotropic vortex at $\gamma=3$. The crosses designate the location of the critical points of the instantaneous corotating streamfunction. The time frame are $t=0,40,80$, etc. 


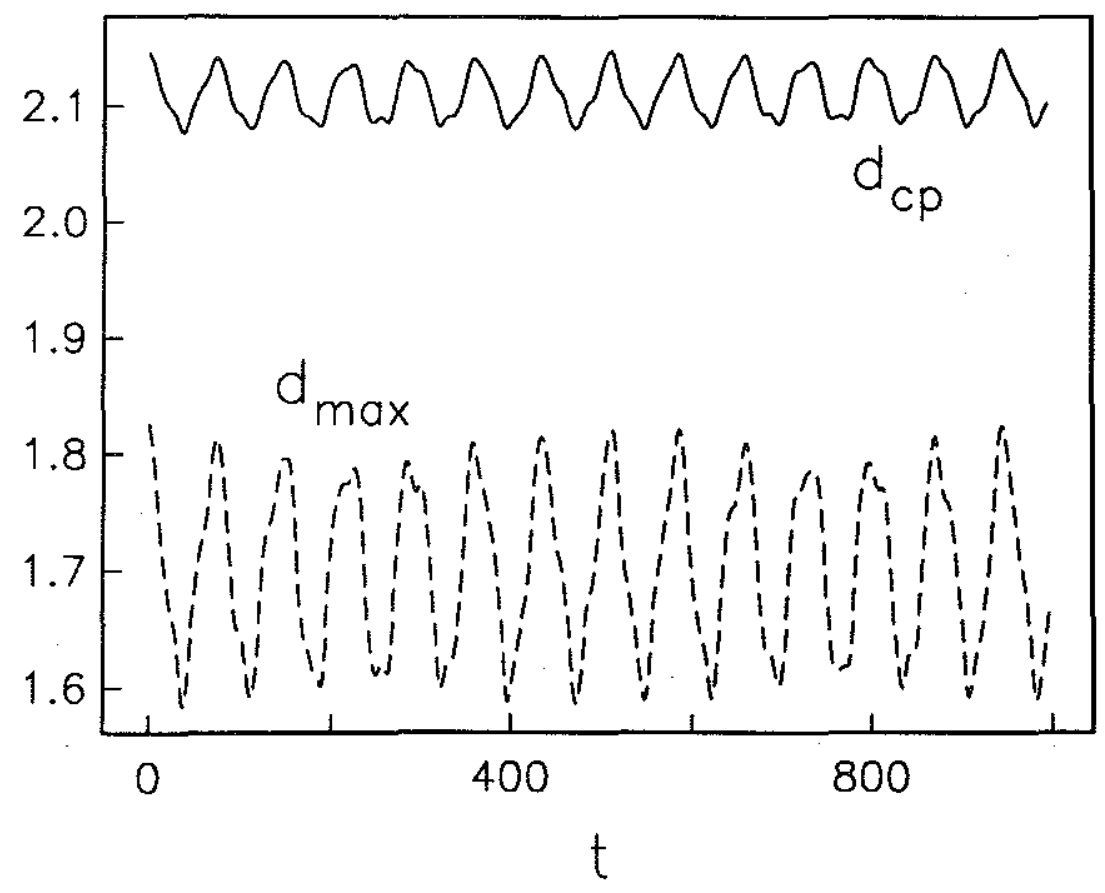

FIGURE 4.12. Same as Figure 4.5 but for the equivalent barotropic evolution of Figure 4.11 at $\gamma=3$. 


\section{Conclusion}

La dernière démarche de la raison est de reconnaître qu'il y a une infinité de choses qui la surpassent. $\dagger^{\dagger}$

Pascal

The new results presented in this thesis can be grouped into two broad categories. On the one hand a number of fundamental questions in the dynamics of isolated coherent vortex structures have been addressed within the context of the quasigeostrophic two-layer approximation. The problem of coalescence of two vortices in the same layer (merger) or in different layers (alignment) has been studied in detail. The critical distances for merger and alignment were numerically established as functions of the ratio of the vortex to the deformation radius and of the relative thickness of the layers at rest.

The deep connection between the coalescence process and the existence of doubly-connected rotating $V$-states was made explicit by comparing the critical merger and alignment distances with the geometrical properties of these $\mathrm{V}$-states. In general, merger takes place within regions in parameter space where $\mathrm{V}$-states do not exist and also somewhat beyond the existence boundary. Dritschel (1985) has shown that multiply-connected Euler $V$-states tend to be unstable near the existence boundary and we believe that to be the case for two-layer ones as well.

We have also found dipolar translating solutions of the two-layer quasigeostrophic equations, either with one pole in each layer or with both poles in the same layer. Their characteristics, as well as the ones of the rotating states, have helped clarify the peculiar nature of the equivalent barotropic model for motions on scales large compared with the deformation radius. In that limit the dynamics of that model were shown to be very different from those of the two-layer model, and in particular, the process of filamentation was found to be greatly suppressed. Essentially, the Greens' function for potential vorticity anomalies in the upper layer alone is very logarithmic in character (although

The last advance of reason is to recognize that it is surpassed by an infinity of things. 
with different amplitudes, corresponding to different time scales). Only when the lower layer is very deep does the exponential character of the induced velocity become apparent for motions on a scale larger than $L_{R}$.

A new geometrical approach to the understanding of the dynamics of isolated and coherent vortex structures has yielded a number of insights on questions previously lacking simple explanations. Above all, we have shown the importance of the critical (stagnation) points of the comoving streamfunction associated with a vortex structure. These have been found to determine bounds (in parameter space) on the existence and the shape of stationary configurations of vorticity.

We have also shown that they play a crucial role in the filamentation process. We have studied in detail the evolution of a perturbed Kirchhoff ellipse, for which case simple geometrical arguments combined with linear theory have allowed us to derive an analytic expression for the time of filamentation which was found to compare well with numerical simulations. The same geometrical approach has also elucidated the suppression of filamentation for equivalent barotropic motions with a small deformation radius.

Since the principal results of this thesis have been obtained using models with discontinuous vorticity distributions, one may wonder to what extent these results would be found to be qualitatively confirmed in continuous systems. On the basis of the rather extensive investigations on $2 \mathrm{D}$ systems, which have shown the results of contour dynamics to be qualitatively confirmed by studies of more complex continuous systems, we have reasons to believe that, at least qualitatively, the phenomenology of the merger and alignment problems that we have presented here should be sufficiently robust to generalize to continuous systems. However, the quantitative results will be dependent on the smoothness of the vorticity fields.

In the case of $\mathrm{V}$-states, the situation is more difficult, since it may be very hard or impossible to find non-trivial smooth equilibria. We believe nonetheless that smoothed out versions of our solutions represent relatively long-lived structures. Undoubtedly, very delicate matters, such as the precise location of the boundary for existence of equilibria and their stability, are very dependent on the form of the vorticity distribution.

All in all, it is our belief that the contour dynamical calculations presented here should be considered a first step in our understanding of stratified and rotating vorticity dynamics. Because of its computational simplicity and the ease 
at obtaining insights into the flow equilibria and evolution, the contour dynamical approach is very well suited for the rather simple situations considered in this thesis, but it is rather obvious that the computational burden would very easily become unbearable if several layers and many vortices with a number of nested contours were to be considered.

There are a number of important additions to the problems we have treated here that, we believe, deserve future investigation. In the first place the presence of a planetary gradient of vorticity, the beta-effect, will introduce the possibility of radiative interactions and energy transfers (Rossby waves) which are liable to substantially enrich the dynamics of the problems presented here. It would also prove interesting to study a system with a more realistic stratification than two layers of equal depth; several layers (or a continuous stratification) will exhibit dynamics that the simple two layers are unable to capture.

The model we have considered in this thesis, inviscid two-layer quasigeostrophy on the f-plane, is probably much too simplified to allow for a direct comparison with actual geophysical situations. However, it is not unreasonable to believe that at least the gross qualitative conclusions maybe sufficiently robust to allow one to draw some meaningful parallels with the observations.

Thus, for instance, the merger and alignment processes are likely to play significant roles in the highly nonlinear dynamics of the oceanic mesoscale eddy field, where relative vorticity dominates over the planetary one, and for which fully nonlinear f-plane dynamics is a reasonable simplest model. These processes are the fundamental mechanisms that mediate, respectively, the reverse barotropic two-dimensional energy cascade and the barotropic to baroclinic energy conversion.

We would also like to mention the filamentation process, which is probably related, among other things, to the large masses of fluid, often referred to as streamers, that are observed to be entrained and detrained in the vicinity of Gulf Stream rings, and whose presence is probably responsible for the observed near axisymmetry of the latter. This phenomenon is one the clearest geophysical examples of the role played by filamentation in reducing the ellipticity of isolated coherent vortices. 


\section{Addenda}

\section{A. Generalized Kirchhoff Vortices}

Problems worthy

of attack

prove their worth

by hitting back!

Piet Hein

We append here the discovery of a new family of exact time dependent solutions of the two-dimensional Euler equations. They are generalizations of the Kirchhoff solution to $\mathrm{N}$ confocal ellipses. It should be noted that, because the boundaries delimiting the regions of constant vorticity are forced to be elliptical, the values of the vorticity jumps across them are prescribed by the appropriate matching conditions, and cannot be chosen arbitrarily.

It turns out, unfortunately, that the vorticity distributions associated with these vortices are somewhat "odd". In particular, the vorticity changes sign across each interface, and for the 2-contour case, these vortices tend to look rather like elliptical annuli of vorticity, with a core of opposite signed smaller amplitude vorticity.

The linear stability for the 2-contour vortex is also presented. It is an extension of Love's (1893) analysis for the Kirchhoff ellipse. Stable regions in parameter space are found. 


\title{
Generalized Kirchhoff vortices
}

\author{
L. M. Polvani and G. R. Flieri \\ Center for Meteorology and Physical Oceanography, Massachusetts Institute of Technology, Cambridge, \\ Massachusetts 02139
}

(Received 11 September 1985; accepted 28 April 1986)

A family of exact solutions of the Euler equations is presented: they are generalizations of the Kirch hoff vortex to $N$ confocal ellipses. Special attention is given to the case $N=2$, for which the stability is analyzed with a method similar to the one used by Love [Proc. London Math. Soc. 1, XXV 18 (1893) ] for the Kirchhoff vortex. The results are compared with those for the corresponding circular problem.

\section{INTRODUCTION}

A two-dimensional elliptical patch of homogeneous in viscid fluid of uniform vorticity rotating with constant angular velocity is an exact solution of the Euler equations; it is called a Kirchhoff vortex.' Its vorticity $Q$ and angular velocity $\omega$ are related by

$$
Q=\left[(a+b)^{2} / a b\right] \omega,
$$

where $a$ and $b$ are, respectively, the major and minor axes of the ellipse. The system is stable to small perturbations provided $a<3 b{ }^{2}$ We present in this work a family of exact solutions which are generalizations of the Kirchhoff vortex to $N$ confocal eilipses, and we investigate the stability for the special case $N=2$.

In terms of the usual Cartesian coordinates $(x, y)$, the elliptical coordinates $(\rho, \theta)$ needed to obtain an analytic solution are implicitly defined by

$x=c \cosh \rho \cos \theta, \quad y=c \sinh \rho \sin \theta$.

The lines $\rho=$ const define confocal ellipses whose foci are located on the $x$ axis at $x= \pm c$.

\section{GENERALIZED KIRCHHOFF VORTICES}

Consider now the following distribution of vorticity $Q$ (Fig. 1):

$$
\begin{aligned}
& Q=0, \text { for } \rho>\rho_{1}, \\
& Q=Q_{j}, \text { for } \rho_{j}>\rho>\rho_{j+1}, j=1, \ldots, N-1, \\
& Q=Q_{n}, \text { for } \rho<\rho_{N} .
\end{aligned}
$$

The stream function must then satisfy

$$
\begin{aligned}
& \nabla^{2} \psi_{0}=0, \text { for } \rho>\rho_{1}, \\
& \nabla^{2} \psi_{j}=Q_{j}, \text { for } \rho_{j}>\rho>\rho_{i+1}, j=1, \ldots, N-1, \\
& \nabla^{2} \psi_{N}=Q_{N}, \text { for } \rho<\rho_{N},
\end{aligned}
$$

which in elliptical coordinates becomes

$$
\begin{gathered}
\left(\frac{\partial^{2}}{\partial \rho^{2}}+\frac{\partial^{2}}{\partial \theta^{2}}\right) \psi_{0}=0, \quad \rho>\rho_{1}, \\
\left(\frac{\partial^{2}}{\partial \rho^{2}}+\frac{\partial^{2}}{\partial \theta^{2}}\right) \psi_{j}=\frac{1}{2} Q_{j} c^{2}(\cosh 2 \rho-\cos 2 \theta), \\
\rho_{j}>\rho>\rho_{j+1}, \quad j=1, \ldots, N-1, \\
\left(\frac{\partial^{2}}{\partial \rho^{2}}+\frac{\partial^{2}}{\partial \theta^{2}}\right) \psi_{N}=\frac{1}{2} Q_{N} c^{2}(\cosh 2 \rho-\cos 2 \theta), \\
\rho<\rho_{N} .
\end{gathered}
$$

Since the whole system is in uniform rotation with angular velocity $\omega$, we must impose the condition that the stream function with respect to a rotating reference frame, $\psi-\frac{1}{2} \omega r^{2}$, must be invariant along the boundaries, i.e.,

$\frac{\partial}{\partial \theta}\left(\psi-\frac{1}{2} \omega\left(x^{2}+y^{2}\right)\right)=0 \quad$ on $\rho=\rho_{j}, \quad j=1, \ldots, N$.

This condition eliminates all homogeneous solutions except the ones proportional to $\cos 2 \theta$. The solution to the inhomogeneous system (1) which satisfies (2) is easily found to be

$$
\begin{aligned}
\psi_{0}= & 1 \omega c^{2} e^{-2\left(\rho-\rho_{1}\right)} \cos 2 \theta+(\Gamma / 2 \pi) \rho, \text { for } \rho>\rho_{1}, \\
\psi_{j}= & \mid Q_{j} c^{2}(\cosh 2 \rho+\cos 2 \theta)+B_{j} \sinh 2 \rho \cos 2 \theta \\
& +C_{j} \cosh 2 \rho \cos 2 \theta+(\Lambda, 2 \pi) \rho+\psi_{j}^{0}, \\
& \text { for } \rho_{j}>\rho>\rho_{j+1}, j=1, \ldots, N-1, \\
\psi_{N}= & 1 Q_{N} c^{2}(\cosh 2 \rho+\cos 2 \theta) \\
& +A \cosh 2 \rho \cos 2 \theta+\psi_{N}^{\circ}, \text { for } \rho<\rho_{N}
\end{aligned}
$$

where

$$
\begin{aligned}
& B_{j}=-\frac{1}{4} c^{2}\left(\omega-\frac{1}{2} Q_{j}\right) \frac{\sinh \left(\rho_{j}+\rho_{j+1}\right)}{\cosh \left(\rho_{j}-\rho_{j+1}\right)}, \\
& C_{j}=\frac{1}{4} c^{2}\left(\omega-\frac{1}{2} Q_{j}\right) \frac{\cosh \left(\rho_{j}+\rho_{j+1}\right)}{\cosh \left(\rho_{j}-\rho_{j+1}\right)}, \\
& A=\left\{c^{2}\left(\omega-\frac{1}{2} Q_{N}\right)\left(\cosh 2 \rho_{N}\right)^{-1} .\right.
\end{aligned}
$$

The constants $\psi_{j}^{\circ}$ can be chosen to make $\psi$ continuous across the boundaries; since our matching conditions are posed in terms of the velocities-i.e., the flow in the rotating frame must be tangent to the boundaries-these constants are im-

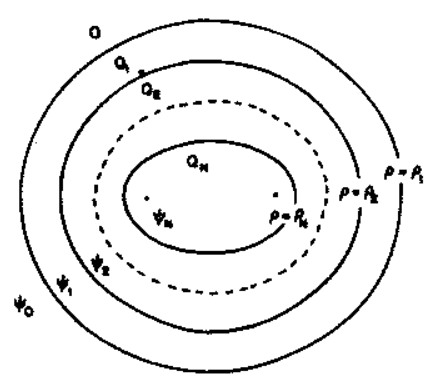

FIG. 1. A schematic drawing of a generalized Kirchhor vortex. 
material. The $Q$,'s and $\Lambda$, 's are determined by requiring continuity of the tangential velocities:

$$
\frac{\partial}{\partial \rho} \psi_{1-1}=\frac{\partial}{\partial \rho} \psi_{1} \quad \text { on } \rho=\rho, \quad \text { for } j=1, \ldots, N
$$

which guarantees continuity of pressure. This condition yields

$\Gamma=\Lambda_{1}+\pi a_{1} b_{1} Q_{3}$,

$\Lambda_{j-1}=\Lambda_{j}+\pi a_{j} b,\left(Q_{j}-Q_{j-1}\right), \quad j=2, \ldots, N-1$,

$\Lambda_{N-1}=\pi a_{N} b_{N}\left(Q_{N}-Q_{N-1}\right)$,

and

$$
\begin{aligned}
& Q_{j}=2 \omega\left[1+i \quad j^{j+1} \operatorname{coth}\left(\rho_{j}-\rho_{j+1}\right)\right] \\
& j=1, \ldots, k-1, \\
& Q_{N}=2 \omega\left[1+(-1)^{v+1} \operatorname{coth} 2 \rho_{N}\right]
\end{aligned}
$$

where $a_{j}$ and $b_{j}$ are, respectively, the major and minor axes of the $j$ th ellipse, and are related to the $\rho$ 's by

$$
a_{j}=c \cosh \rho_{j} \text { and } b_{j}=c \sinh \rho_{j} .
$$

It is easy to show that the $N=1$ case corresponds to the Kirchhoff vortex. Using (6) one can rewrite ( 3 ) as follows:

$$
\begin{aligned}
\psi_{0}= & \frac{1}{8} \omega c^{2} e^{-2\left(\rho-\rho_{1}\right)} \cos 2 \theta+(\Gamma / 2 \pi) \rho, \\
\psi_{j}= & \frac{1}{8} Q_{j} c^{2}(\cosh 2 \rho+\cos 2 \theta)+\left(\Lambda_{j} / 2 \pi\right) \rho \\
& +(-1)^{j+1} \frac{1}{4} \omega c^{2} \frac{\sinh \left(\rho_{j}+\rho_{j+1}\right)}{\sinh \left(\rho_{j}-\rho_{j+1}\right)}
\end{aligned}
$$

$\times \sinh 2 \rho \cos 2 \theta$

$$
\begin{aligned}
&+(-1)^{\prime} \frac{1}{4} \omega c^{2} \frac{\cosh \left(\rho_{j}+\rho_{j+1}\right)}{\sinh \left(\rho_{j}-\rho_{j+1}\right)} \cosh 2 \rho \\
& \times \cos 2 \theta+\psi_{j}^{\circ}, \quad j=1, \ldots, N-1, \\
& \psi_{N}= \frac{1}{8} Q_{N} c^{2}(\cosh 2 \rho+\cos 2 \theta)+(-1)^{N} 1 \omega c^{2} \\
& \times\left(\sinh 2 \rho_{N}\right)^{-1} \cosh 2 \rho \cos 2 \theta+\psi_{N}^{\rho} .
\end{aligned}
$$

It should be pointed out that once $\omega$ and the $\rho_{j}$ 's $(j=1, \ldots, N)$ have been chosen, the $Q_{j}$ 's follow necessarily from (6) and therefore cannot be set arbitrarily. In particular it is easy to see that $Q_{j}$ and $Q_{j+1}$ always have opposite signs, and that $Q$, has the sign of $\omega$. A further interesting property of these solutions is that the total circulation $\Gamma$ is determined uniquely by the angular velocity $\omega$ and the size $\rho_{1}$ of the outer ellipse. Indeed it can be shown from (5) that

$$
\Gamma=\pi \omega\left(a_{1}+b_{1}\right)^{2}=\pi \omega c^{2} e^{-2 \rho_{1}}
$$

for all values of $N$ and $\rho_{j}$ 's $(j>1)$.

\section{STABILITY OF THE $N=2$ VORTEX}

We now turn our attention to the special case $N=2$, for which the stream function is given by

$$
\begin{aligned}
\psi_{0}= & 1 \omega c^{2} e^{-2\left(\rho-\rho_{1}\right)} \cos 2 \theta+\frac{1}{2} \omega c^{2} e^{-2 \rho_{1}} \rho, \\
\psi_{1}= & \frac{1}{8} Q_{1} c^{2}(\cosh 2 \rho+\cos 2 \theta)+\frac{1}{2} a_{2} b_{2}\left(Q_{2}-Q_{1}\right) \rho \\
& +\frac{1}{4} \omega c^{2} \frac{\sinh \left(\rho_{1}+\rho_{2}\right)}{\sinh \left(\rho_{1}-\rho_{2}\right)} \sinh 2 \rho \cos 2 \theta \\
& -\frac{1}{4} \omega c^{2} \frac{\cosh \left(\rho_{1}+\rho_{2}\right)}{\sinh \left(\rho_{1}-\rho_{2}\right)} \cosh 2 \rho \cos 2 \theta+\psi_{1}^{0}, \\
\psi_{2}= & -\frac{1}{\alpha} Q_{2} c^{2}(\cosh 2 \rho+\cos 2 \theta)+\frac{1}{4} \omega c^{2} \\
& \times\left(\sinh 2 \rho_{2}\right)^{-1} \cosh 2 \rho \cos 2 \theta+\psi_{2}^{\circ},
\end{aligned}
$$

with

$$
Q_{i}=2 \omega\left[1+\operatorname{coth}\left(\rho_{1}-\rho_{2}\right)\right]
$$

and

$$
Q_{2}=2 \omega\left(1-\operatorname{coth} 2 \rho_{2}\right) \text {. }
$$

Of the four parameters $\rho_{1}, \rho_{2}, \omega$, and $c$ only the first two are important in determining the shape, structure, and stability of the vortices.

Contours of the quantity $Q_{1} / Q_{2}$ in the $\left(\rho_{1}, \rho_{2}\right)$ plane are plotted in Fig. 2. Also shown in that figure are the shapes of the vortices for three typical values of $\rho_{1}$ and $\rho_{2}$.

In order to investigate the stability of the steady state (8) we use a method similar to the one in Ref. 2 . We denote by $\phi_{0}, \phi_{1}$, and $\phi_{2}$ the perturbation stream functions. The perturbed boundaries of the ellipses are given by

$$
\rho=\rho_{1}+\rho_{i}^{\prime}(\theta) \text { and } \rho=\rho_{2}+\rho_{2}^{\prime}(\theta) \text {. }
$$

Both the $\phi$ 's and the $\rho$ ' 's are understood to be infinitesimal quantities. The $\phi$ 's must satisfy Laplace's equation together with the following conditions:

$$
\begin{aligned}
& \frac{\partial}{\partial \rho}\left(\psi_{0}+\phi_{0}\right)=\frac{\partial}{\partial \rho}\left(\psi_{1}+\phi_{1}\right) \quad \text { on } \rho=\rho_{1}+\rho_{1}^{\prime}, \\
& \frac{\partial}{\partial \rho}\left(\psi_{1}+\phi_{1}\right)=\frac{\partial}{\partial \rho}\left(\psi_{2}+\phi_{2}\right) \quad \text { on } \rho=\rho_{2}+\rho_{2}^{\prime}, \\
& \frac{\partial}{\partial \theta}\left(\psi_{0}+\phi_{0}\right)=\frac{\partial}{\partial \theta}\left(\psi_{1}+\phi_{1}\right) \text { on } \rho=\rho_{1}+\rho_{1}^{\prime}, \\
& \frac{\partial}{\partial \theta}\left(\psi_{1}+\phi_{1}\right)=\frac{\partial}{\partial \theta}\left(\psi_{2}+\phi_{2}\right) \text { on } \rho=\rho_{2}+\rho_{2}^{\prime}, \\
& \frac{D}{D t}\left[\rho-\rho_{1}-\rho_{1}^{\prime}(\theta)\right]=0 \text { on } \rho=\rho_{1}+\rho_{1}^{\prime}, \\
& \frac{D}{D t}\left[\rho-\rho_{2}-\rho_{2}^{\prime}(\theta)\right]=0 \text { on } \rho=\rho_{2}+\rho_{2}^{\prime},
\end{aligned}
$$

where

$$
\begin{aligned}
& \frac{D}{D t}=\frac{\partial}{\partial t}+J\left(\psi+\phi-\frac{1}{2} \omega r^{2}\right), \\
& J(f g)=\frac{\partial f}{\partial x} \frac{\partial g}{\partial y}-\frac{\partial f}{\partial y} \frac{\partial g}{\partial x} .
\end{aligned}
$$

The last condition ensures that the deformed ellipses always contain the same fluid. It is possible to show that the above three conditions guarantee continuity of pressure at the boundaries.

Since the ф's are solutions of Laplace's equation we can immediately write 


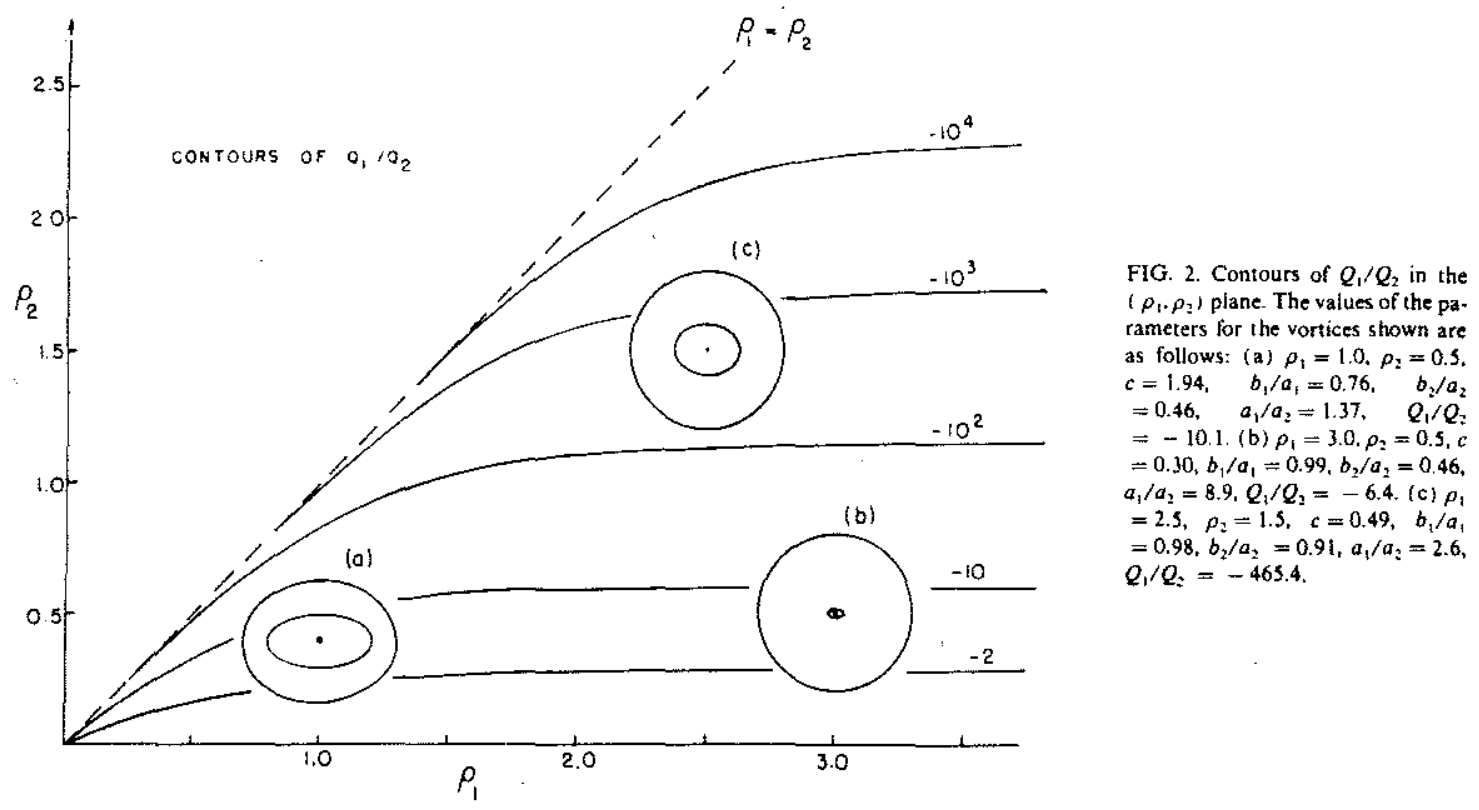

$$
\begin{aligned}
\phi_{0}= & \sum_{m}\left(A_{m} e^{-m \rho} \cos m \theta+B_{m} e^{-m \rho} \sin m \theta\right) \\
\phi_{1}= & \sum_{m}\left(C_{m} \cosh m \rho \cos m \theta+D_{m} \sinh m \rho \cos m \theta\right. \\
& +E_{m} \cosh m \rho \sin m \theta+F_{m} \\
& \times \sinh m \rho \sin m \theta)+\phi_{1}^{0} \\
\phi_{2}= & \sum_{m}\left(G_{m} \cosh m \rho \cos m \theta\right. \\
& \left.+H_{m} \sinh m \rho \sin m \theta\right)+\phi_{2}^{0} .
\end{aligned}
$$

Conditions (9) and (10) can be combined to eliminate four of the eight unknowns. After some algebra they can be shown to simply reduce to

$$
\begin{aligned}
& \sum_{m} m e^{m \rho_{1}}\left[\left(C_{m}+D_{m}\right) \cos m \theta+\left(E_{m}+F_{m}\right) \sin \theta\right] \\
& \quad+\frac{1}{2} Q_{1}\left(\frac{\rho_{1}^{\prime}}{h_{1}^{2}}\right)=0, \\
& \sum_{m} m\left(\frac{D_{m}}{\cosh m \rho_{2}} \cos m \theta-\frac{E_{m}}{\sinh m \rho_{2}} \sin m \theta\right) \\
& \quad-\frac{1}{2}\left(Q_{2}-Q_{1}\right)\left(\frac{\rho_{2}^{\prime}}{h_{2}^{2}}\right)=0,
\end{aligned}
$$

where we have defined

$$
h_{i}^{-2}=c^{2}\left(\cosh 2 \rho_{i}-\cos 2 \theta\right), \quad i=1,2 .
$$

In a similar way (11) must be Taylor expanded about the unperturbed boundaries. After much algebra we obtain

$$
\begin{aligned}
& \left.\frac{\partial}{\partial \theta} \phi_{1}\right|_{\rho_{1}}=-\frac{\partial}{\partial t}\left(\frac{\rho_{1}^{\prime}}{h_{1}^{2}}\right)-\frac{1}{2} \omega \frac{\partial}{\partial \theta}\left(\frac{\rho_{1}^{\prime}}{h_{1}^{2}}\right), \\
& \left.\frac{\partial}{\partial \theta} \phi_{2}\right|_{\rho_{2}}=-\frac{\partial}{\partial t}\left(\frac{\rho_{2}^{\prime}}{h_{2}^{2}}\right)+\frac{1}{2} \omega \frac{\partial}{\partial \theta}\left(\frac{\rho_{2}^{\prime}}{h_{2}^{2}}\right) .
\end{aligned}
$$

Since $\rho_{1}^{\prime}$ and $\rho_{2}^{\prime}$ always appear in combination with $h_{1}^{-2}$ and $h_{2}^{-2}$ we can expand them together as follows:

$$
\begin{aligned}
& \left(\rho_{1}^{\prime} / h_{1}^{2}\right)(\theta)=\sum_{m}\left(\alpha_{m} \cos m \theta+\beta_{m} \sin m \theta\right), \\
& \left(\rho_{2}^{\prime} / h_{2}^{2}\right)(\theta)=\sum_{m}\left(\gamma_{m} \cos m \theta+\delta_{m} \sin m \theta\right) .
\end{aligned}
$$

Upon substitutions of (15) into (13) it immediately follows that

$$
\begin{aligned}
& \alpha_{m}=-\left(2 m / Q_{1}\right)\left(C_{m}+D_{m}\right) e^{m \rho_{1}}, \\
& \beta_{m}=-\left(2 m / Q_{1}\right)\left(E_{m}+F_{m}\right) e^{m \rho_{1}},
\end{aligned}
$$

and

$$
\begin{aligned}
\gamma_{m} & =\frac{2 m}{\left(Q_{2}-Q_{1}\right)} \frac{D_{m}}{\cosh m \rho_{2}}, \\
\delta_{m} & =\frac{-2 m}{\left(Q_{2}-Q_{1}\right)} \frac{E_{m}}{\sinh m \rho_{2}} .
\end{aligned}
$$

The final step is the substitution of (12) and (15) into (14). Setting the coefficients of $\cos m \theta$ and $\sin m \theta$ to zero yields a system of four homogeneous equations in four unknowns. If the coefficients in (12) are assumed to be proportional to $e^{-i \sigma}$, the equation for $\sigma$ is obtained by requiring that a nontrivial solution exist, i.e., that the determinant of the system vanish. This leads to an equation of the form:

$$
(\sigma / \omega)^{4}-A\left(m, \rho_{1}, \rho_{2}\right)(\sigma / \omega)^{2}-B\left(m, \rho_{1}, \rho_{2}\right)=0,
$$

where the coefficients $A$ and $B$ are given by

$$
\begin{aligned}
& A=\alpha \beta-\zeta \xi+\gamma(2 \alpha-2 \beta+\zeta+\xi-\gamma), \\
& B=\alpha \beta\left(\gamma^{2}-\zeta \xi\right)-\gamma\left(\alpha^{2} \xi+\beta^{2} \xi\right),
\end{aligned}
$$

and 


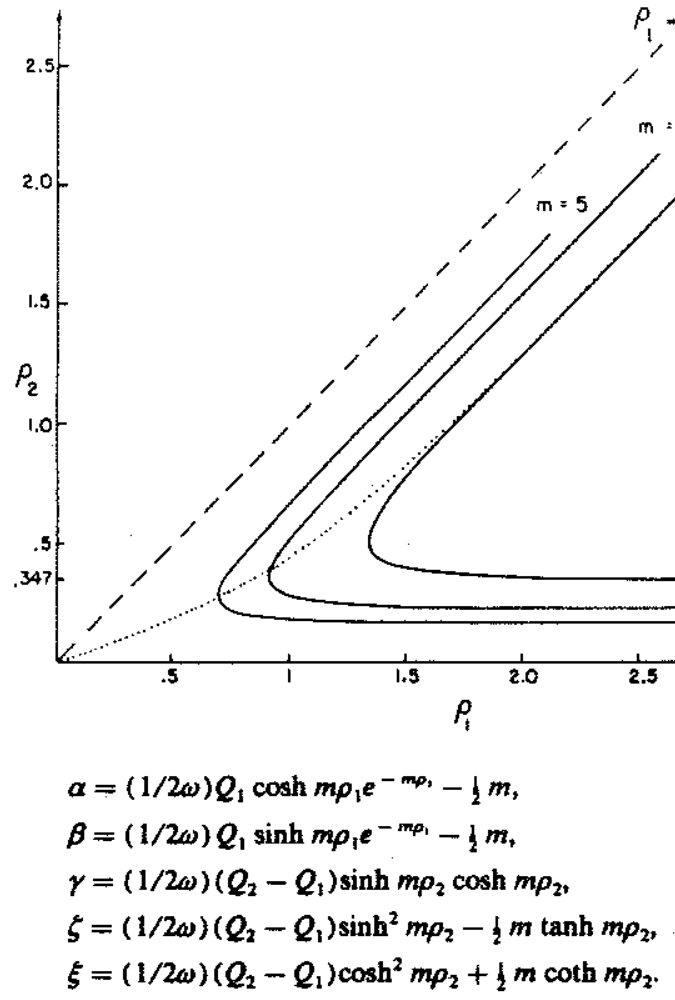

From the form of (16) it is clear that $\omega$ plays no role in determining the stability of the vortex. Furthermore it is easy to show that for stability the following conditions must be satisfied:

$$
A>0, B<0, \text { and } A^{2}+4 B>0 \text {. }
$$

Because of the complexity of the coefficients $A$ and $B$ one has to resort to numerical methods in order to determine the shape of the critical curves in the $\left(\rho_{1}, \rho_{2}\right)$ plane for each mode $m$; they are shown in Fig. 3 . The following results have been established:

(1) The modes $m=1$ and $m=2$ are stable (or neutral) for all values of $\rho_{1}$ and $\rho_{2}$ as was the case for the Kirchhof vortex $(N=1)$.

(2) In the limit of very large $\rho_{1}$ the vortex becomes unstable if $\rho_{2}<0.347$ which corresponds to $b_{2} / a_{2}<1$, in agreement with Love's result for the Kirchhof vortex.

(3) In general, given an inner ellipse of size $\rho_{2}>0.347$ the system will be stable for all $m$ 's provided the size of the outer ellipse exceeds some critical value. For very large values of $\rho_{1}$ and $\rho_{2}$ (i.e., for virtually circular boundaries) the vortex will become unstable for $\left(\rho_{1}-\rho_{2}\right)<0.7$.

Finally, we compare our results with the linear stability theory of Ref. 3 for the circular problem. To each point in the $\left(\rho_{1}, \rho_{2}\right)$ plane we associate a unique pair of concentric circles of uniform vorticity; if the inner one has radius 1 and vorticity 1 , and the outer one radius $r$ and vorticity $q$, we establish a one-to-one correspondence between $\left(\rho_{1}, \rho_{2}\right)$ and $(r, q)$ by choosing
FIG. 3. Stability curves for $N=2$ for the modes $m=3,4,5$. The dotied curve is calculated from Flierl's theory for circular vortices for $m=3$ as described in the text.

$$
r^{2}=\frac{\sinh 2 \rho_{1}}{\sinh 2 \rho_{2}} \text { and } q=\frac{Q_{1}}{Q_{2}},
$$

which means that the outer to inner ratios of area and vorticity are the same for the concentric circles and the confocal ellipses.

The stability curve for the $m=3$ mode is shown in Fig. 3. As expected, it agrees well with the curve for the confocal ellipse for large values of $\rho_{1}$ and $\rho_{2}$. The circular theory breaks down for $\rho_{2}<1.2$, which corresponds to $b_{2} / a_{2}<0.83$.

In conclusion, we point out that two quite different kinds of instabilities can be identified from the curves of Fig. 3. The lower branch of these curves (which is asymptotic to $\rho_{2}=0.347$, i.e., $b_{2} / a_{2}=\{$ for the mode $m=3$ ) represents a Love-type instability due to excessive eilipticity of the inner boundary. The upper branch, on the contrary, is really a Rayleigh-type shear instability associated with the fact that the vorticity gradient does not have a unique sign throughout the vortex.

\section{ACKNOWLEDEMENTS}

L. M. Polvani is a student in the MIT/Woods Hole Oceanographic Institution Joint Program in Physical Oceanography.

This work was funded in part by the Ofice of Naval Research under Grant N00014-80-0273. L. M. Polvani was supported, in part, by a Postgraduate Fellowship from the Natural Sciences and Engineering Research Council of Canada.

'H. Lamb, Hydrodyenior, 6th ed. (Dover, New York, 1932)

${ }^{2}$ A. E. H. Love Proc. London Math. Soc. 1, XXV 18 (1893).

See National Technical Informetion Service Document No. AD Al49386/S (Woods Hole Oeenographic Intitution Technical Report, WHOI-84-44). Copies may be ordered from the National Technicai Information Service, Springeteld, Virginin, 22161. The price is $\$ 22.95$ plus \& 53.00 hundling fee. All orders mase be prepaid. 


\title{
B. The 2-Level Rankine Vortex: Critical Points \& Stability
}

\author{
L'example souvent n'est qu'un miroir trompeur. ${ }^{\dagger}$
}

\section{Corneille}

It has been shown in Chapter 4 how the linear stability of the Rankine vortex and the Kirchhoff ellipse are totally unrelated to the location of the critical points of their corotating streamfunction. The central idea is the following: linear stability theory is applicable in the limit of vanishing amplitude, and therefore, as long as the critical points are not located exactly on the vortex boundaries, a linear (i.e. infinitesimal) perturbation will be unaware of their presence.

To demonstrate this point we consider in this section a situation in which it is possible to have critical points actually coinciding with the vorticity discontinuities, and show that, when this happens, the linear stability of the vortex is connected with the location of the critical points of the corotating streamfunction.

This simplest example is provided by the 2-level Rankine vortex, for which the axisymmetric vorticity $\nabla^{2} \psi$ versus the radial distance $r$ from the origin is schematically illustrated in Figure 7.1. It is composed of two circular nested regions of constant vorticity surrounded by irrotational flow. Without loss of generality, we have chosen the inner vorticity and the outer radius to be equal to 1 , so that only two parameters appear in this problem: $q^{\ddagger}$, the ratio of outer to inner vorticity, and $R$, the ratio of inner to outer radius (note that $0<R<1$ ).

This vorticity distribution, being axisymmetric, is an exact nonlinear stationary solution of the Euler equations. Its linear stability has been investigated by a number of authors (see Flierl 1988, for the latest and most complete version), but their choices of parameters are quite inappropriate for the present context. We briefly summarize the analysis to introduce the notation.

† Example is often but a lying mirror.

$¥$ We confine our attention here to the range $-1<q<1$, where the connection between linear stability and the position of the critical points is established. 
The streamfunction of the unperturbed vortex is the solution of:

$$
\begin{array}{rll}
0 & \text { for } & r>1 \\
\nabla^{2} \psi=q & \text { for } & R<r<1 \\
1 & \text { for } & r<R
\end{array}
$$

with $\psi_{r}$ continuous at $r=1$ and $r=R$. It is easy to show that :

$$
\psi=\begin{array}{lll}
(1 / 2)\left[q+(1-q) R^{2}\right] \log r & \text { for } & r>1 \\
(1 / 2)(1-q) R^{2} \log r+(1 / 4) q r^{2} & \text { for } & R<r<1 \\
(1 / 4) r^{2} & \text { for } & r<R
\end{array}
$$

We now consider an infinitesimal perturbation $\varphi$ of mode $m$ on this basic state which, following Lamb's (1945) analysis of the (one-level) Rankine vortex, we write:

$$
\varphi=\begin{array}{lll}
(A+B) r-m \cos (m \vartheta-\Omega t) & \text { for } & r>1 \\
\left(A-m+B r^{m}\right) \cos (m \vartheta-\Omega t) & \text { for } & R<r<1 \\
\left(A R^{-2 m}+B\right) r m \cos (m \vartheta-\Omega t) & \text { for } & r<R
\end{array}
$$

The form (7.3) is chosen to satisfy continuity of $\varphi_{\vartheta}$ at $r=1$ and $r=R$. Next we must impose continuity in the radial derivative of total streamfunction $\Psi=\psi+\varphi$. If the outer and inner perturbed vortex boundaries are located at $1+\eta_{1}(\vartheta, t)$ and $R+\eta_{R}(\vartheta, t)$ respectively, it is simple to see that continuity of $\Psi_{r}$ implies continuity of the quantity $\eta \psi_{r r}+\varphi_{r}$ at $r=1$ and $r=R$. The last condition to be enforced is the kinematic constraint that ensures that fluid does not cross the vorticity boundaries:

$$
\eta_{t}+(1 / r) \psi_{r} \eta_{\vartheta}=-(1 / r) \varphi_{\vartheta} \quad \text { at } \quad r=1 \text { and } r=R
$$

These four boundary conditions, together with the choice

$$
\eta_{1}=\mathrm{H}_{1} \cos (m \vartheta-\Omega \mathrm{t}) \quad \text { and } \quad \eta_{\mathrm{R}}=\mathrm{H}_{\mathrm{R}} \cos (m \vartheta-\Omega \mathrm{t})
$$

are used to eliminate the four constants $A, B, H_{1}$ and $H_{R}$ and yield, after much algebra, the dispersion relation for the normal modes of the problem:

$$
\begin{aligned}
& (2 \Omega)^{2}-(2 \Omega)\left[q+(m-1)(1+q)+m(1-q) R^{2}\right] \\
& +(m+q-1)\left[(m-1) q+m(1-q) R^{2}\right]-q(1-q) R^{2 m}=0
\end{aligned}
$$

Note that this is a quadratic in $\Omega$, as is expected from the presence of two contours. Thus for a given choice of the parameters $(q, R)$ two eigenmodes exist for the same 
$m$ value. It is also straightforward to show that the ratio of the amplitudes of the deviations of the boundaries from the unperturbed circles is given by the simple expression:

$$
\frac{\mathrm{H}_{1}}{\mathrm{H}_{\mathrm{R}}}=\frac{(\mathrm{m}-1)-2 \Omega}{\mathrm{qR}^{\mathrm{m}-1}}
$$

Lets us now concentrate on the case $m=2$, for which the dispersion relation reduces to:

$$
\begin{aligned}
& (2 \Omega)^{2}-(2 \Omega)\left[1+2 q+2(1-q) R^{2}\right] \\
& +q(1+q)+2\left(1-q^{2}\right) R^{2}-q(1-q) R^{4}=0
\end{aligned}
$$

We determine first the regions of stability in the $(q, R)$ parameter space. The discriminant $\Delta$ for the quadratic (7.5) can be shown to be given by:

$$
\begin{aligned}
\Delta & =1-4 R^{2}(1-q)\left(1-R^{2}\right) \\
& \equiv 1-(1-q) g(R)
\end{aligned}
$$

where it is a simple matter to prove that the function $g(R)$ reaches the maximum value of 1 at $R=1 / \sqrt{2}$ in the interval $0<R<1$.

For $q>0$ it is clear that $\Delta>0$ for all $R$, so that all solutions are stable. Consider now the appearence of unstable regions in $R$ as $q$ is made negative. If we let $q \equiv \zeta$ with $0<\zeta<<1$, it is easy to show that the unstable vortices are found in a window of width proportional to $\zeta^{1 / 2}$ centered around $R=1 / \sqrt{ } 2$.

The interesting result here is that, as was shown in Section 4.1, for the vortex with $R=1 / \sqrt{ } 2$ and $q \equiv 0, m=2$ perturbations on the inner contour located at $R=1 / \sqrt{2}$ have critical points associated with them located at $r=1$, the radial position of the outer contour. Therefore, as $q$ is made negative, the window of instability in $R$ opens around the value for which the critical points of the inner vorticity distribution are coincident with the outer vorticity boundary .

The location of the critical points can obviously be calculated exactly for this problem. To lowest order in the amplitude of the pertubations the radial location $r_{c}$ of the critical points is found by solving:

$$
\left.\partial_{r} \psi\right|_{r=r_{c}} \equiv V\left(r_{c}\right)=(1 / 2) \Omega r_{c}
$$


where $\psi$ is given in (7.2) and $\Omega$ is easily obtained from (7.5). Because $V(r)$ is always positive for $r<R$, but can be negative for $r>R$ when $q<0$, there can be as many as three values of $r_{c}$ that solve (7.7).

The expression for $\psi$ to be used in (7.7) depends on whether $R<r<1$ or $r 1$. It is simple to show that:

$$
r_{c}=R\left\{\frac{1-q}{\Omega-q}\right\}^{1 / 2} \quad \text { when } \quad R<r<1
$$

and

$$
r_{c}=\left\{\frac{q\left(1-R^{2}\right)+R^{2}}{\Omega}\right\}^{1 / 2} \quad \text { when } \quad r>1
$$

It should be clear that it is not possible to have $r_{c}<R$. The values for $\Omega$ easily are obtained from (7.5):

$$
\Omega_{ \pm}=(1 / 4) b \pm(1 / 4)\left[b^{2}-4 c\right]^{1 / 2}
$$

where

$$
b=1+2 q+2(1-q) R^{2} \quad \text { and } \quad c=q(1+q)+2\left(1-q^{2}\right) R^{2}-q(1-q) R^{4}
$$

Because these expressions are rather complicated, it is useful to evaluate them numerically to understand the parametric dependences.

We start by showing the real parts of $\Omega$ as functions of $R$ for $q=0.5,0.1,0.01$, $-0.01,-0.1$ and -0.5 in Figure 7.2 ; the solid line corresponds to $\Omega_{+}$and the dashed one to $\Omega_{-}$. Note how, as $q$ is decreased and passes through zero, the two roots coalesce first for $q=0$ at $R=1 / \sqrt{ } 2$, and how the window of instability widens as $q$ is made more negative.

The radii of the critical points are plotted versus $R$ for the same values of $q$ in Figure 7.3. It is interesting to note that, for the stable cases with $q>0$, the two critical points associated with the two values of $\Omega$ are located one between $r=R$ and $r=1$ (for $\Omega_{+}$) and the other one outside $r=1$ (for $\Omega_{-}$). As $q$ is made smaller the two critical points coalesce on the outer contour $(r=1)$ at $R=1 / \sqrt{ } 2$ for $q=0$. Thus the appearance of instability is associated with the coalescence of the two critical points on the outer vorticity boundary.

When $\operatorname{Im}(\Omega) \neq 0$, of course, the notion of the "corotating frame" becomes ambiguous because the fields are not stationary in any frame of reference. The values plotted in Figure 7.3 for the unstable cases are obtained by solving (7.7) with the real part of $\Omega$ alone. The interesting result is that, for $q<0$, both critical points are either inside the outer boundary, for $R<1 / \sqrt{ } 2$, or outside it, for $R>1 / \sqrt{ } 2$, and 
that the region of instability seems to be associated with a range in $R$ for which the critical points are located near the outer boundary.

[The dotted lines appearing in the $q<0$ cases of Figure 7.3 are associated with a third critical point that appears for sufficiently small $R$, and is associated with

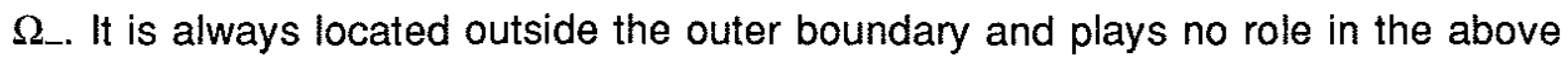
arguments. It is simple to show that it exists only for $q<0$ and $R<\{q /(q-1)\}$. ] 


\section{Tripolar Coherent Euler Vortices}

Rara avis in terris nigroque simillima cygno. ${ }^{\dagger}$

Juvenal

In numerical simulations of two-dimensional turbulence as well as in laboratory experiments with rotating fluids, the frequency of appearance of isolated coherent vortex structures is observed to decrease very rapidly with the increasing number of poles. Thus, for instance, the number of dipoles emerging in decaying turbulence simulations (McWilliams 1984, Babiano et al. 1986) compared to the number of monopoles is found to be quite small.

It is for this reason that the first examples of tripolar coherent vortices were only first observed fairly recently. To the best of our knowledge, the earliest reference in the literature is due to Leith (1981) and the first clear picture was provided by Legras et al. (1988) who observed a tripole spontaneously emerge in one of their high resolution spectral simulations of two-dimensional turbulence. By analogy with classical electromagnetism, it should be clear that tripoles are in fact quadrupole distributions of vorticity (recall that poles must be powers of 2), in the same way as "modons" are dipoles.

In this addendum we report on a new family of finite-area constant-vorticity equilibria ( $V$-states) for the two-dimensional Euler equations that are the piecewise-constant analogs of the tripole reported by Legras et al. The generic shape of these vortices is shown in Figure 7.4. They are composed of three regions of vorticity: the two outer regions (the "satellite" vortices) have constant vorticity equal to 1 , while the central vortex has vorticity $\gamma<0$ (vortices with $\gamma>0$ probably also exist, but they are not being considered here).

The parameter space for this problem is three dimensional, since $\gamma$ as well as the two radii $a$ and $b$ (see Figure 7.4) can be specified arbitrarily (note that $0<a<1$ and $0<b<1)$. The question to be addressed here is the following: for what value of $(\gamma, a, b)$ do tripolar solutions exist? For fixed values of $\gamma$ and $a$, we find that, as $b$ is increased from to 0 to 1 , convergence fails when the central vortex becomes too close to the satellites; when this happens the vortices are very different in shape from circles, and curvatures of both signs occur on their contours. Whether

† A rare bird on this earth, like nothing so much as a black swan. 
this failure is due to the inadequacy of our algorithm to handle very contorted shapes or to the existence of a limiting $V$-state at some value of $b \in(0,1)$ is a question that we have not examined in detail (refer to the discussion in Chapter 3 ).

The algorithm we have used to determine the tripolar solutions presented here is an adaptation of the second order scheme of Wu et al. (1984) to the geometry of this problem. By symmetry the nodes can be placed in the upper righthand quadrant alone as shown in Figure 7.5 , with $4 \mathrm{M}$ nodes on the central vortex and $2 \mathrm{~N}$ on the satellites. The algorithm proceeds in a way very similar to the one described in section 1.5.1; the novelty here is that we need to insure that all the contours rotate at the same angular velocity $\Omega$, which is calculated after each iteration using the expression:

$$
\Omega=\left[1-a^{2}+b^{2}-R_{1}^{2}\right]^{-1}\left[\sum_{i=1}^{M}+\sum_{i=M+2}^{M+N+1}\right]\left\{v_{i+1 / 2}\left(x_{i+1}-x_{i}\right)-u_{i+1 / 2}\left(y_{i+1}-y_{i}\right)\right\}
$$

where the labelling of the nodes is shown in Figure 7.5. Two tridiagonal systems (cf. Section 1.5.1) must be solved, one for the satellites and one for the central vortex. The analogs of (1.12) can be shown to be given by:

$$
F_{i+1 / 2}=\frac{u_{1 / 2} \sin \theta_{i+1}-v_{i+1 / 2} \cos \theta_{i+1}+(\Omega / 2) R_{i+1}}{u_{i+1 / 2} \sin \theta_{i}-v_{i+1 / 2} \cos \theta_{i}+(\Omega / 2) R_{i}}
$$

$(i=1, \ldots, M)$ for the center vortex and

$$
G_{i+1 / 2} \frac{u_{1 / 2} \sin \theta_{i+1}-v_{i+1 / 2} \cos \theta_{i+1}+(\Omega / 2) R_{i+1}+\Omega x_{0} \cos \theta_{i+1}}{u_{i+1 / 2} \sin \theta_{i}-v_{i+1 / 2} \cos \theta_{i}+(\Omega / 2) R_{i}+\Omega x_{0} \cos \theta_{i}}
$$

$(i=M+2, \ldots, N+M+2)$ for the satellites, where $x_{0} \equiv(1-a) / 2$. It is important to note that the intercept of the contour of the central vortex with the $y$-axis $\left(R_{1}\right)$ is not fixed and, together with the angular velocity $\Omega$, is an unknown to be solved for.

We now present a few solutions to illustrate the kinds of behavior observed. In Figure 7.6 we show the states at $\gamma=-2, a=0.7$ and $b=0.1,0.2,0.3,0.4$. No convergence was achieved for $b>0.4$. Note how, as the satellites approach the central vortex, they become more elongated to oppose its shear and maintain an equilibrium position. In Figure 7.7 the states for $\gamma=-3, a=0.5$ and $b=0.1,0.2$ and 0.3 . The last value of $b$ for which the algorithm converged shows the presence of $a$ 
slightly negative curvatures on the satellite vortices; they are due to the greater strength of the central vortex and become very evident for the last case shown here, the $V$-states at $\gamma=-4, a=0.3$ and $b=0.1$ and 0.2 , shown in Figure 7.8.

It perhaps is interesting to note that the value of $\Omega$ changes sign between $b=0.1$ and $b=0.2$, which implies the existence of nonrotating tripolar configurations. Tripoles with zero circulation are of special interest because the velocity field they generate decreases very rapidly away from them. For a nonrotating point vortex tripole, is is easy to show that that the velocity field decreases like $1 / r^{3}$ for large $r$, compared with a $1 / \mathrm{r}$ dependence for a point vortex monopole, and a $1 / \mathrm{r}^{2}$ for a point vortex dipole). This would mean that such coherent vortex structures are almost "invisible", in the sense that their presence is felt only at very short distances.

We have only briefly investigated the stability of the above solutions, and we have found stable examples as well as unstable ones. In Figure 7.9 we show the evolution of a perturbed $\gamma=-2, a=0.6$ and $b=0.3 \mathrm{~V}$-state. We have chosen a quite simple and at the same time quite general perturbation: we displace one satellite towards the central vortex at $t=0$. The example of Figure 7.9 suggest stability, as is evidenced by the fact that the vorticity field retains its tripolar nature after two full turnaround periods, and the satellites merely oscillate around the central vortex while all three vortex boundaries undergo pulsations in their shapes.

An unstable evolution is presented in Figure 7.10 , for a perturbed $\gamma=-4$, $a=0.6$ and $b=0.2$ tripole. The interesting result could be summarized by saying that an unstable tripole may "decay" into two dipoles. We wish to emphasize that this result is not confined to the piecewise-constant inviscid situation. Pseudospectral simulations (Polvani and Carton, 1988) reveal a similar behavior in a continuous and dissipative context.

Another possible avenue for decay is for the vorticity field to axisymmetrize into a "shielded vortex", i.e. an axisymmetric monopole of vorticity surrounded by vorticity of the opposite sign. One such example is shown Figure 7.11 where a perturbed unstable $\gamma=-4, a=0.3$ and $b=0.2$ tripolar $V$-state is shown to decay into a very nearly axisymmetric shielded vortex.

We conclude by pointing out that these decay modes can, vice-versa, become ways by which tripoles are generated. Thus Flierl (priv. comm.) has recently observed (with pseudosprectral simulations) that an unstable perturbed shielded vortex may decay into a tripole, and Polvani and Carton (1988), also 
using spectral algithms, have shown how tripoles can emerge from the superposition of two dipoles.

Such "two-way" processes are not really a novelty in two-dimensional incompressible vortex dyanmics. Dritschel (1986) has shown that the ellipse and the doubly-connected rotating $\mathrm{V}$-states are also related in a similar way. The richeness of the behavior observed with tripoles is due to the presence of both positive and negative vorticity, and we believe that a number of other decay/formation processes for dipoles and tripoles still remain to be uncovered. 


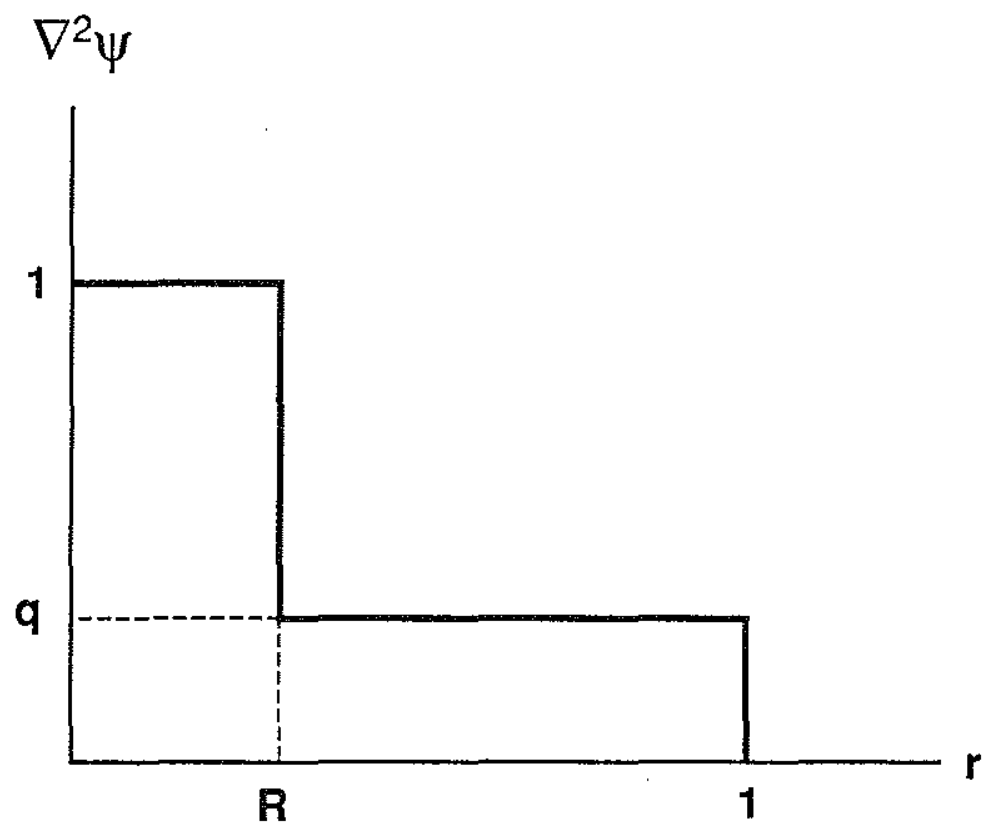

FIGURE 7.1. The vorticity as a function of radius for the 2-contour Rankine vortex. 

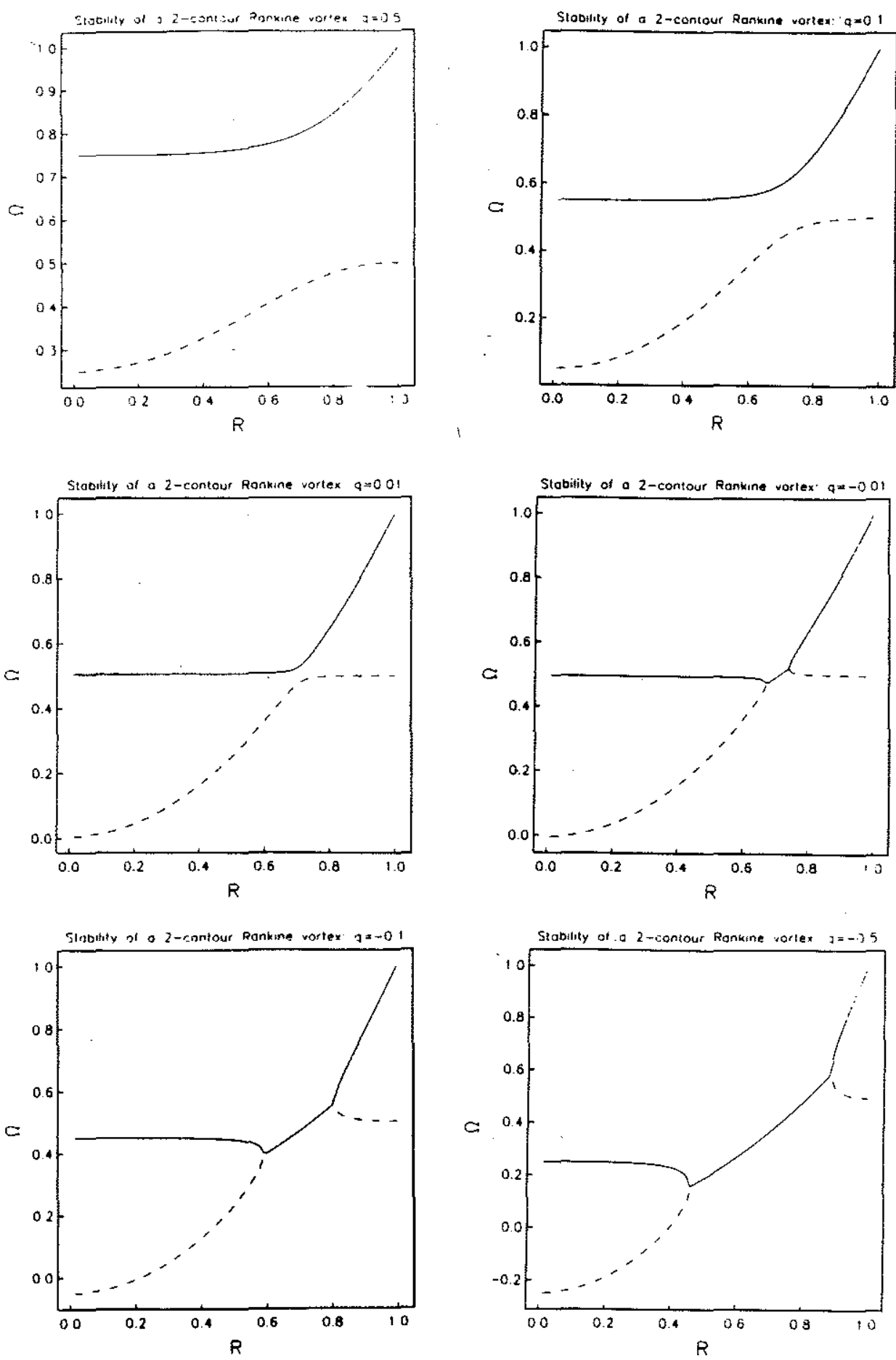

FIGURE 7.2. The angular velocities of the normal modes of the 2-contour Rankine vortex as functions of $R$ for $q=0.5,0.1,0.01,-0.01,-0.1$ and -0.5 . The solid (dashed) line corresponds to $\Omega_{+}(\Omega)$. The 

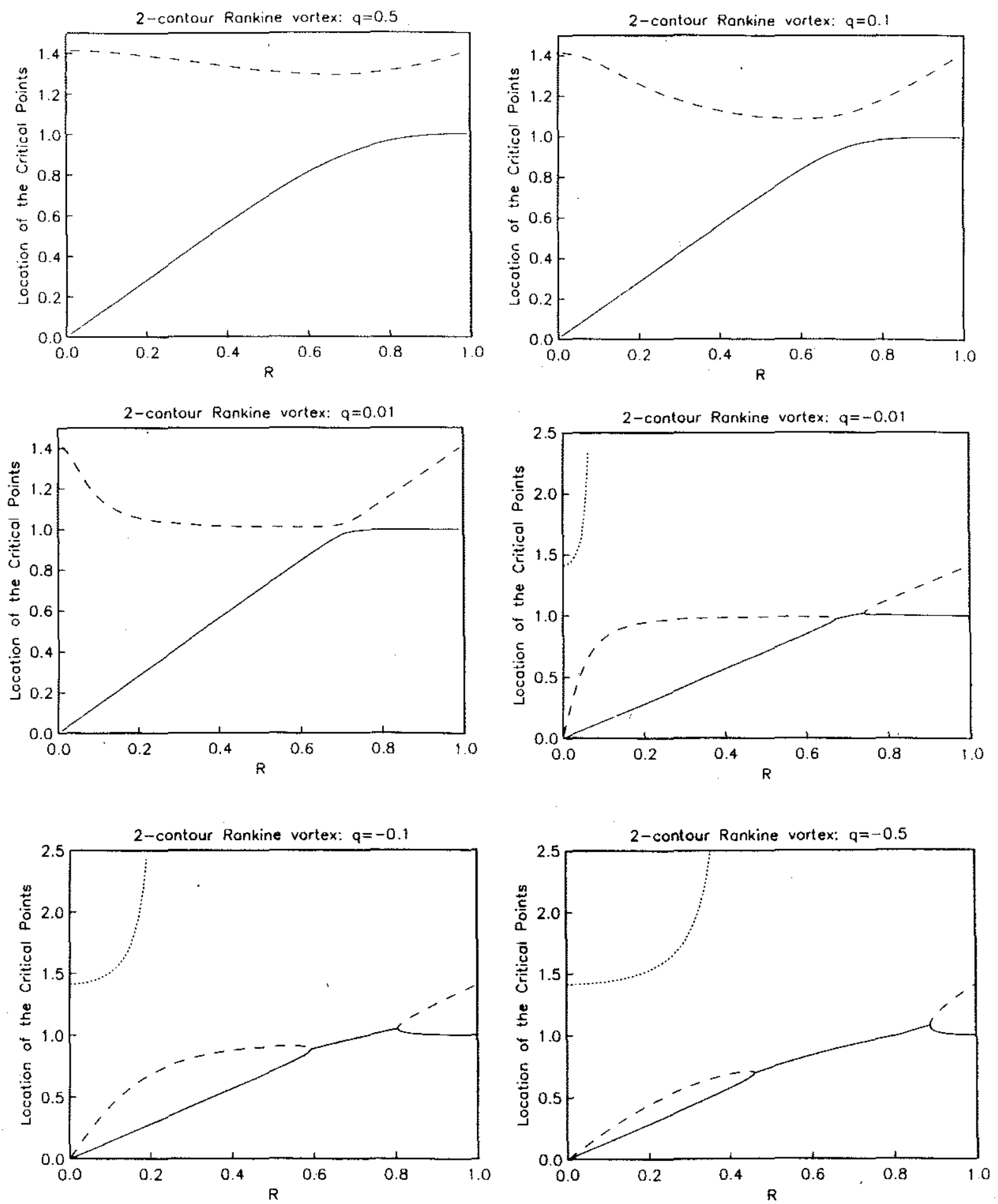

FIGURE 7.3. The location of the critical circles for the normal modes of the 2-contour Rankine vortex as functions of $R$ for $q=0.5,0.1,0.01,-0.01,-0.1$ and -0.5 . The solid (dashed) line corresponds to $\Omega_{+}\left(\Omega_{-}\right)$. The dotted line that appears for $q<0$ is associated with $\Omega$. 


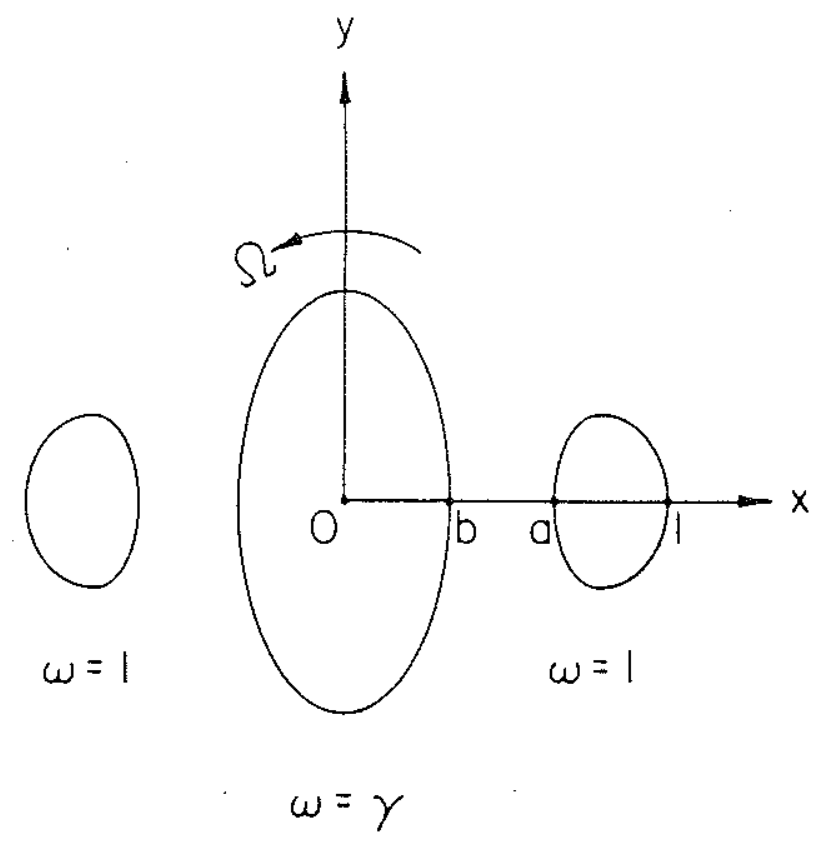

FIGURE 7.4. A schematic drawing of a tripolar Euler V-state. The vorticity $\omega \equiv \nabla^{2} \psi$ is constant inside the vortices and zero outside. The three parameter of this problem are $a, b$ and $\gamma$ (not to be confused with the inverse radius of deformation!). 


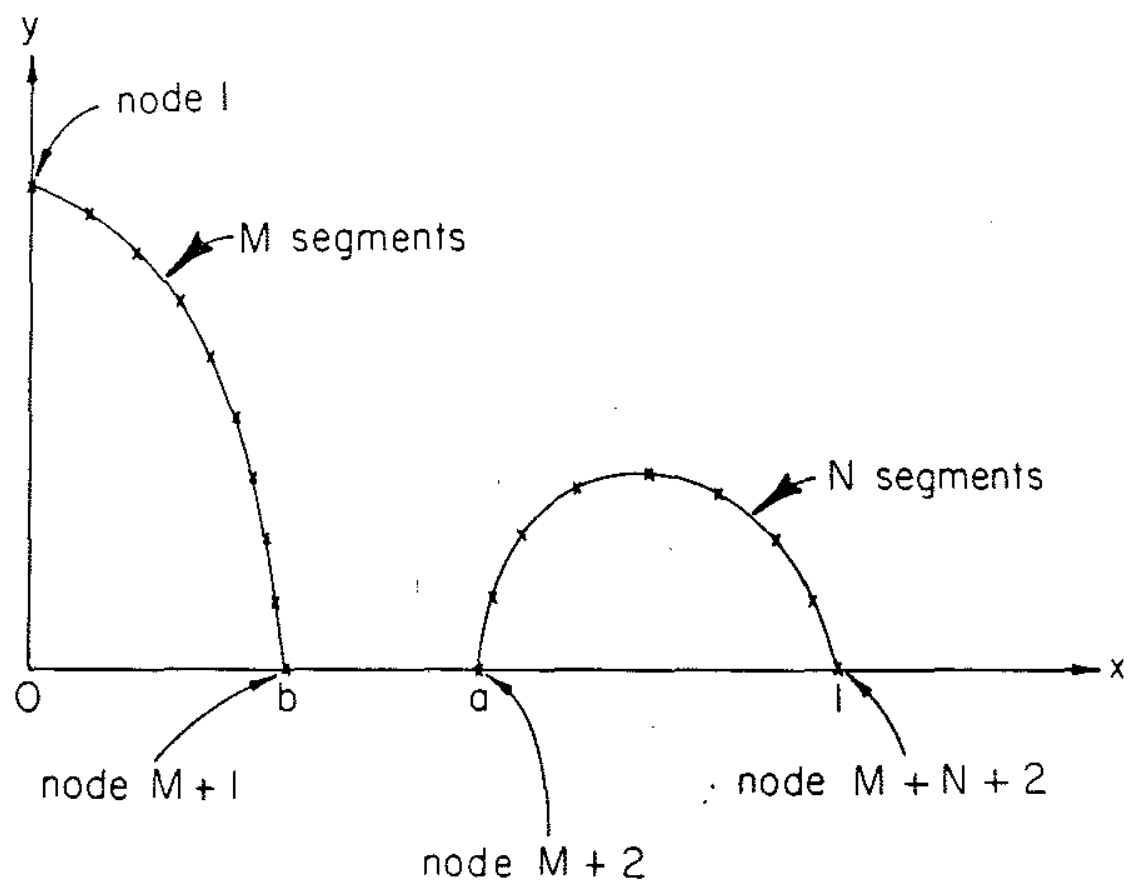

FIGURE 7.5. Arrangement of the nodes for the algorithm used in the determination of the tripolar Euler V-states. Note that the position of nodes $\mathrm{M}+1, \mathrm{M}+2$ and $\mathrm{N}+\mathrm{M}+2$ are fixed, but the location of node 1 is free to change during the iteration. 


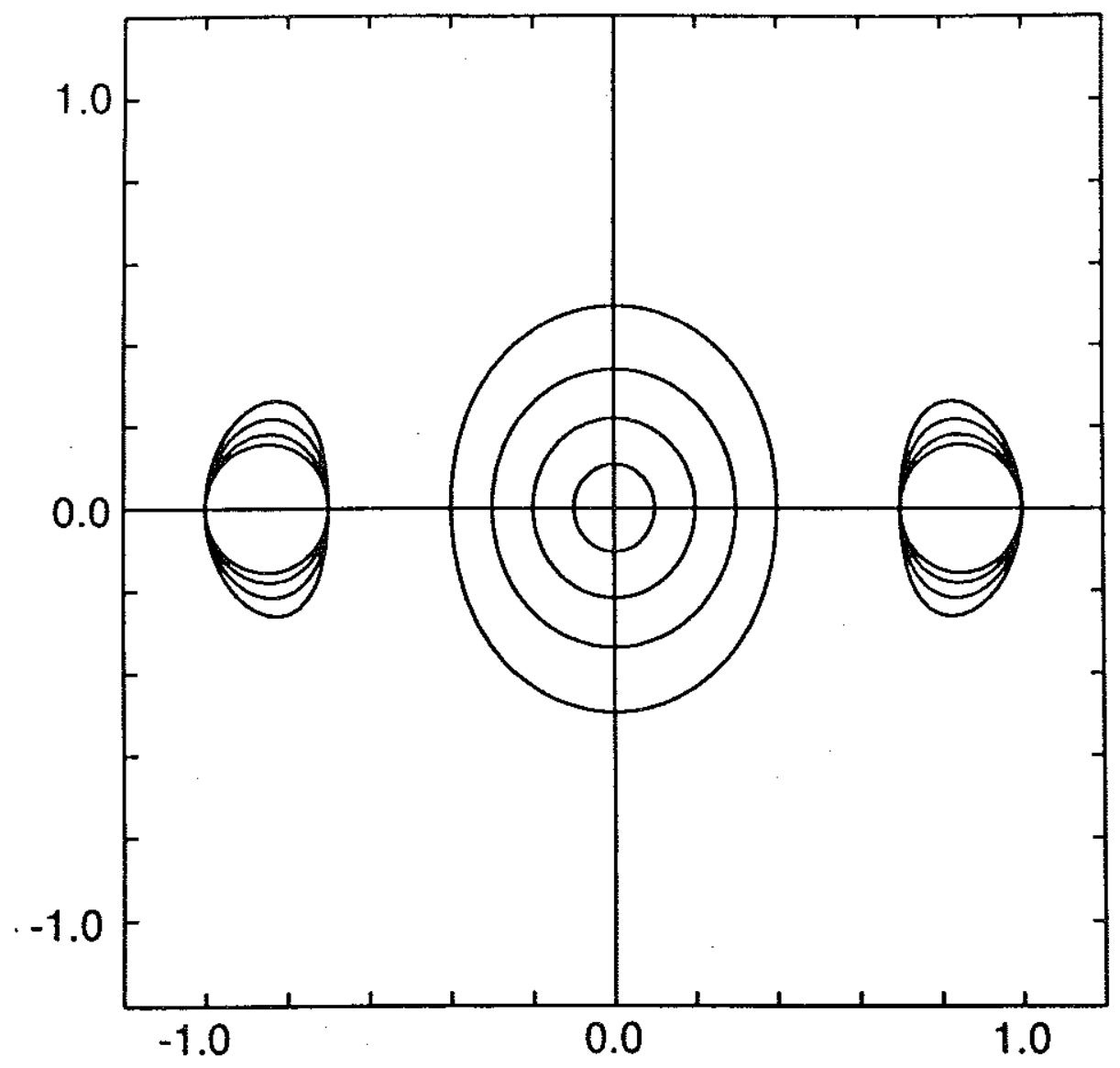

b $2 \pi \Omega$

0.10 $-0.042$

0.20 $-0.318$

0.30 $-0.807$

0.40

$-1.611$

Figure 7.6 Tripolar $V$-state solutions for $\gamma=-2.0, a=0.7$ and $b=0.1,0.2,0.3$ and 0.4 . As $b$ increases the satellites become more elongated. 


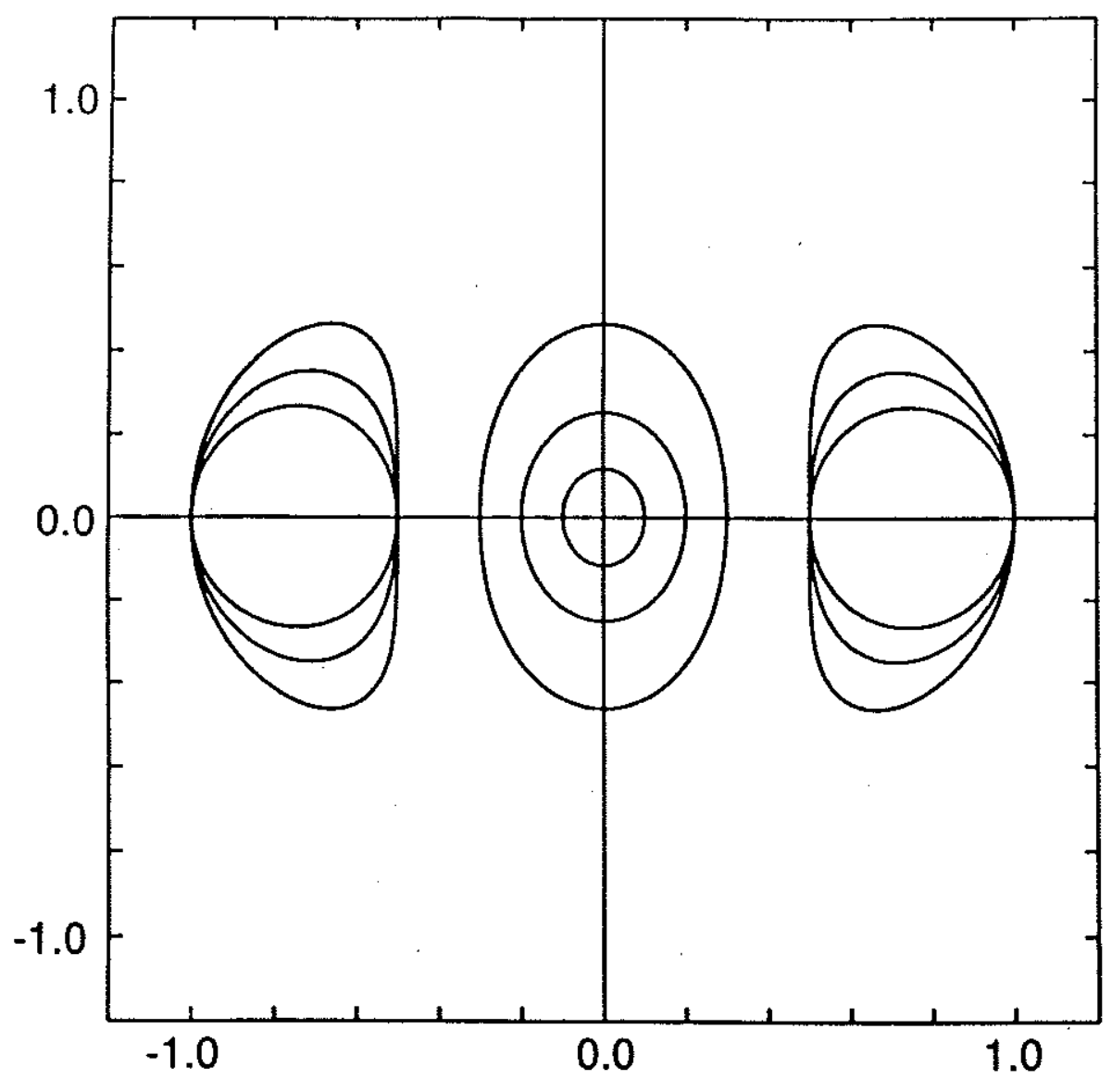

b

$2 \pi \Omega$

0.10

$-0.008$

0.20

$-0.587$

0.30

$-0.1911$

Figure 7.7 Tripolar $V$-state solutions for $\gamma=-3.0, a=0.5$ and $b=0.1,0.2$ and 0.3 . Note the incipient negative curvature on the satellites of the V-state at $b=0.3$. 


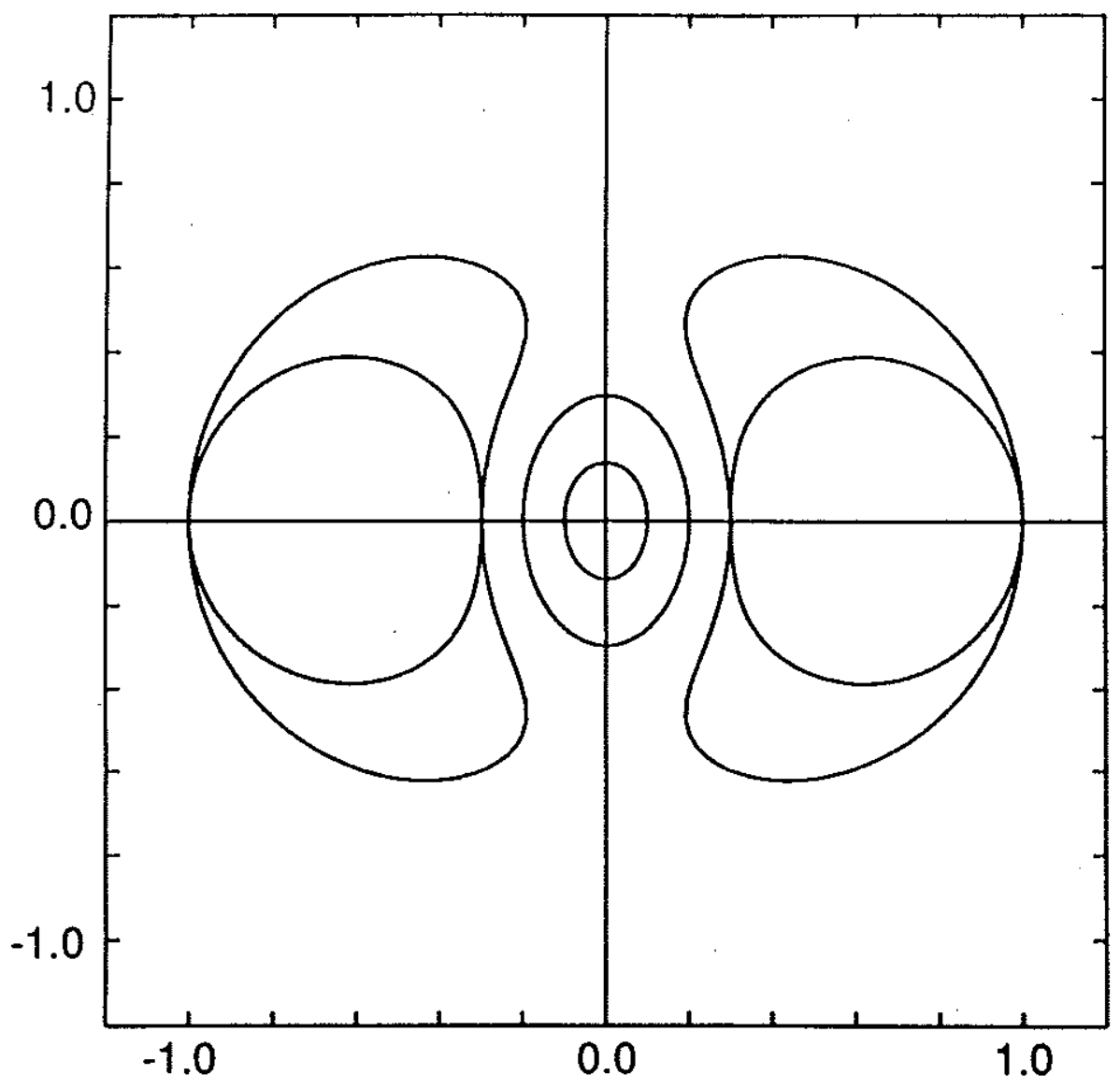

b

$2 \pi \Omega$

0.10

0.115

0.20

$-0.658$

Figure 7.8 Tripolar $V$-state solutions for $\gamma=-4.0, a=0.3$ and $b=0.1$ and 0.2 . Note that there exists a nonrotating $(\Omega=0)$ solution for $0.1<b<0.2$. 


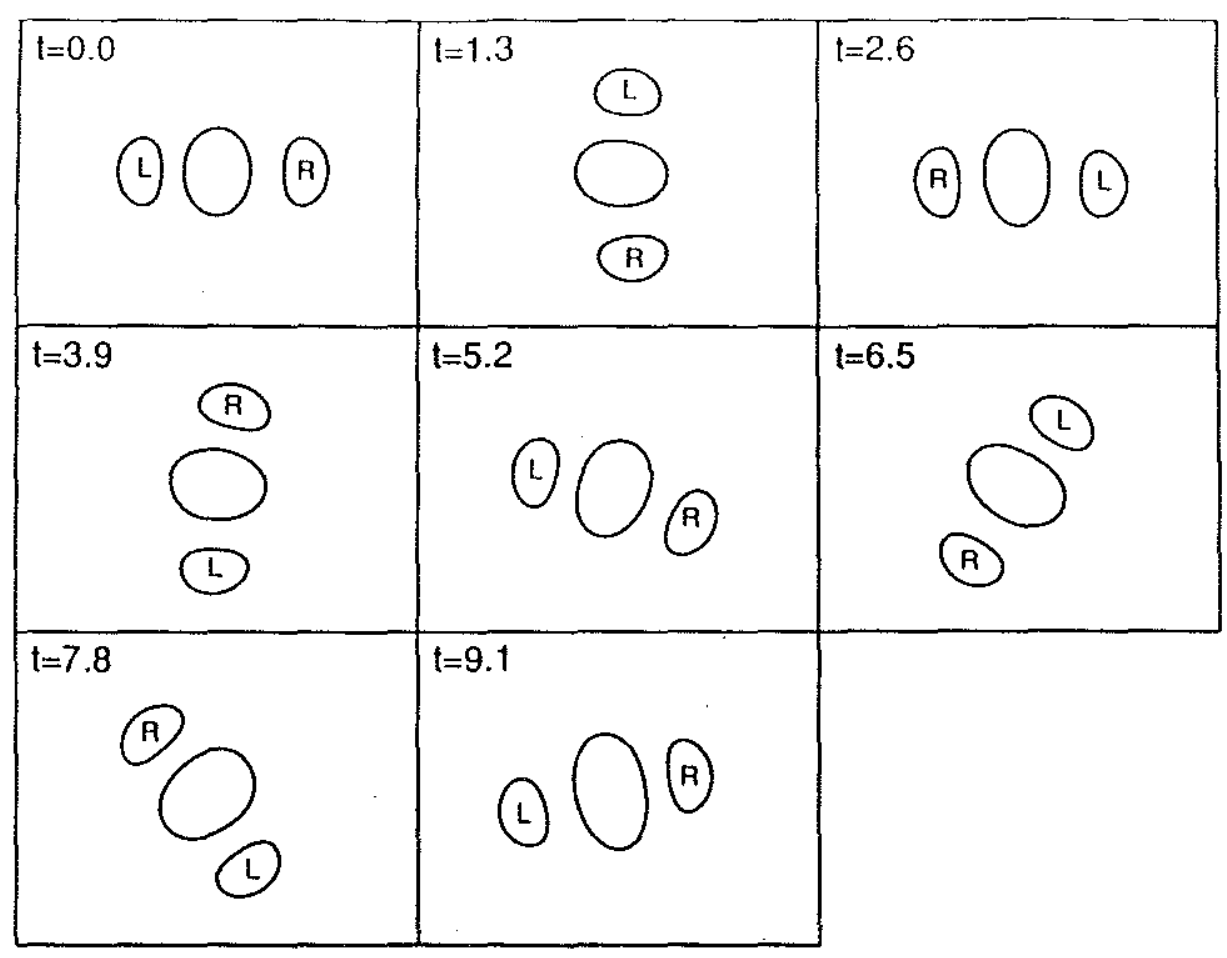

FIGURE 7.9. The evolution of a perturbed $\gamma=-2, a=0.6$ and $b=0.3$ tripolar $V$-state. At $t=0$ the left satellite was displaced a distance 0.1 to the right. 


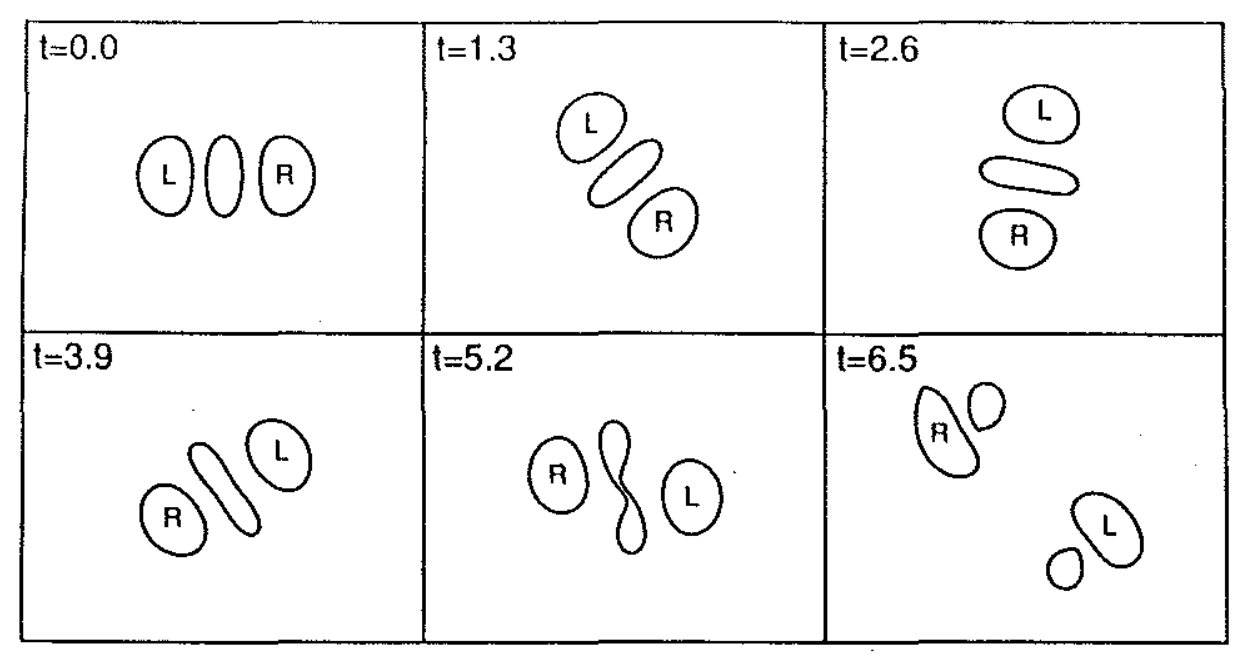

FIGURE 7.10. The evolution of a perturbed $\gamma=-2, a=0.4$ and $b=0.2$ tripolar $V$-state. At $t=0$ the left satellite was displaced a distance 0.05 to the right. 


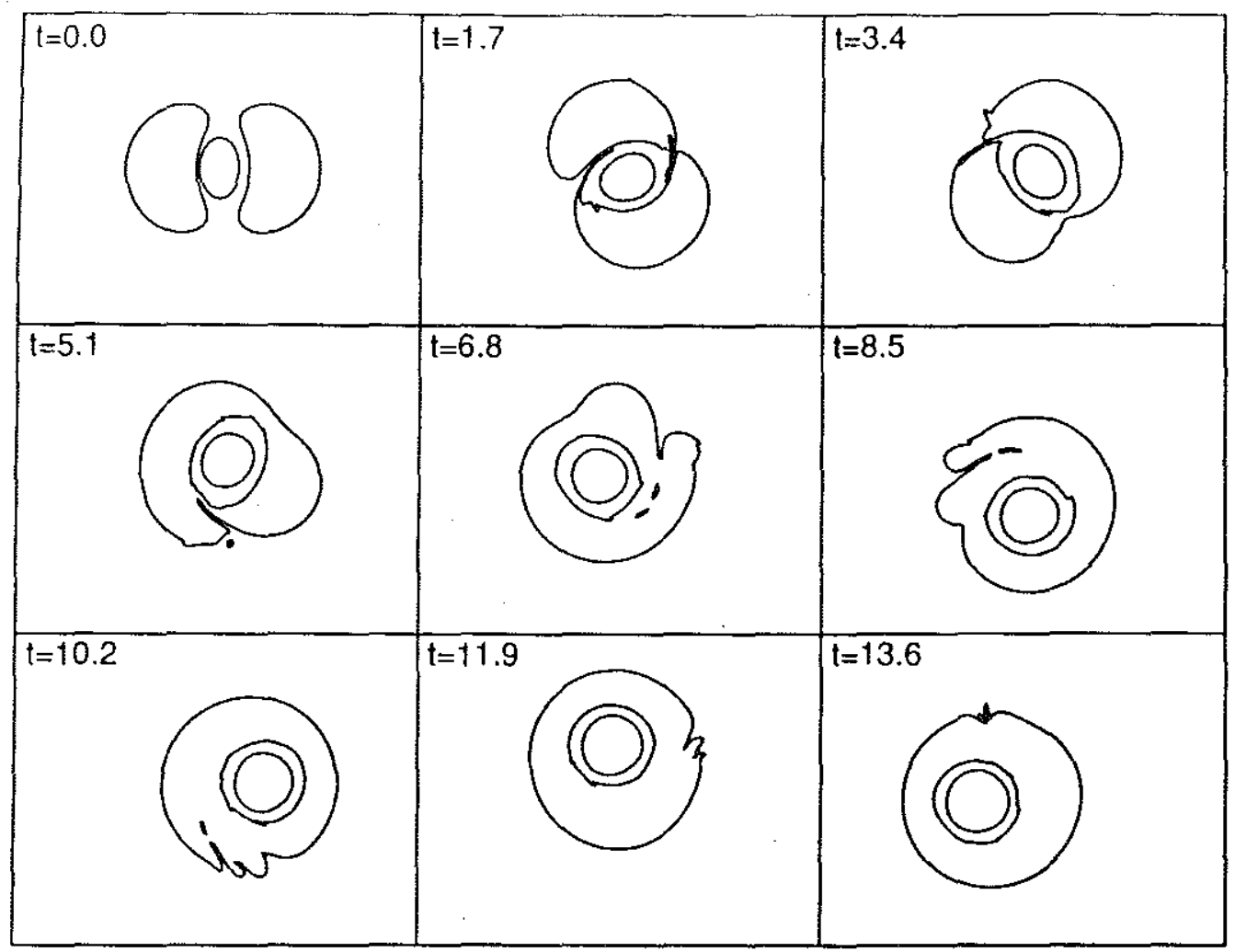

FIGURE 7.11. The evolution of a perturbed $\gamma=-2=4, a=0.4=3$ and $b=0.2$ tripolar $V$ state. At $t=0$ the left satellite was displaced a distance 0.07 to the right. 


\section{Appendices}

Celui qui veut faire des grandes choses

doit penser profondement aux détails. ${ }^{\dagger}$

Valéry

\section{Appendix A}

\section{High Order Schemes for Moment Evaluation}

We describe here in some detail the fourth order algorithms we have used to diagnose the contour dynamical results obtained throughout the thesis. We start by considering the quantities that need to be calculated. In general, given a closed, simply-connected contour $C$ enclosing a domain $D$ in the $(x, y)$ plane the following numbers are of interest:

$$
\begin{aligned}
& \text { Area }=A=M_{00}=\int_{D} d x d y=-\oint_{C} y d x=\oint_{C} x d y \\
& \bar{x}=M_{10}=A^{-1} \int_{D} x d x d y=-A^{-1} \oint_{C} x y d x=A^{-1} \oint_{C}\left(x^{2} / 2\right) d y \\
& \bar{y}=M_{01}=A^{-1} \int_{D} y d x d y=-A^{-1} \oint_{C}\left(y^{2} / 2\right) d x=A^{-1} \oint_{C} x y d y \\
& M_{20}=A^{-1} \int_{D} x^{2} d x d y=-A^{-1} \oint_{C} x^{2} y d x=A^{-1} \oint_{C}\left(x^{3} / 3\right) d y \\
& M_{02}=A^{-1} \int_{D} y^{2} d x d y=-A^{-1} \oint_{C}\left(y^{3} / 3\right) d x=A^{-1} \oint_{C} x y^{2} d y \\
& M_{11}=A^{-1} \int_{D} x y d x d y=A^{-1} \oint_{C}\left(x^{2} y / 2\right) d x=-A^{-1} \oint_{C}\left(x y^{2} / 2\right) d y
\end{aligned}
$$

† The man who wants to do great things must think very carefully about the details. 
In the same vein, the (mth,nth) moment $M_{m n}$ of a given contour is given by:

$$
M_{m n}=A^{-1} \int_{D} x^{m} y^{n} d x d y
$$

However, in order to be able to interpret geometrically the moments for $m+n>1$, it is convenient to redefine them with respect to the centroid $(\bar{x}, \bar{y})$ by considering the quantites:

$$
J_{m n}=A^{-1} \int_{D}(x-\bar{x})^{m}(y-\bar{y})^{n} d x d y \quad \text { for } m+n>1
$$

For an ellipse with aspect ratio $\lambda t$ and whose major axis is inclined with respect to the $x$-axis by an angle $\varphi$ it can be shown that:

$$
\begin{aligned}
& J_{20}=\frac{A}{4 \pi \lambda}\left[1-\left(1-\lambda^{2}\right) \sin ^{2} \varphi\right] \\
& J_{20}=\frac{A}{4 \pi \lambda}\left[1-\left(1-\lambda^{2}\right) \cos ^{2} \varphi\right] \\
& J_{11}=\frac{A}{8 \pi \lambda}\left[1-\lambda^{2}\right] \sin 2 \varphi
\end{aligned}
$$

Thus, in the same way as when, by calculating its area and centroid, one obtains the radius $(\sqrt{ } A / \pi)$ and the center of the "equivalent circle" of any given contour, from the values of the three second order moments one can, by inverting the above relations, find the aspect ratio $\lambda_{\theta q}$ and the inclination $\varphi_{e q}$ of its "equivalent ellipse", which are given by:

$$
\lambda_{\text {eq }}=\kappa-\left[\kappa^{2}-1\right]^{1 / 2} \quad \text { and } \quad \varphi_{\text {eq }}=\frac{1}{2} \sin ^{-1}\left\{\frac{8 \pi \lambda J_{11}}{\left(1-\lambda^{2}\right) A}\right\}
$$

where

$$
\kappa=\frac{2 \pi}{A}\left[J_{20}+J_{02}\right]
$$

Recall, however, that $\lambda_{e q}$ and $\varphi_{e q}$ can only be interpreted meaningfully when the shape of the contour is not too "far" from an ellipse.

t We have adopted here the convention $0 \leq \lambda \leq 1$, so that a very elongated ellipse corresponds to $\lambda<<1$. 
Having designated the moments as the quantities to be calculated, we now proceed to expound on the fourth order algorithm we have employed to evaluate the $J_{m n}$ 's. The general expression that needs to be computed can put in the form:

$$
M=\oint_{C} F(y) d y
$$

Because nodes that discretize the contour are assumed to be a given set $\left\{\left(x_{i}, y_{i}\right), i=1, N\right\}$, the intervals in dy between any two pairs of successive nodes are usually not equal. We thus first recast the contour integral in a form such that the variable of integration is incremented by a constant amount from one node to the next. The simplest choice for such a variable is the node label $n$, which leads to the expression:

$$
M=\oint_{C} F(y) \frac{d y}{d n} d n \equiv \oint_{C} G(n) d n
$$

Now we split the integral over the entire contour in a sum of the integrals over the intervals between two nodes, and thus write:

$$
M=\sum_{i=1}^{N} \int_{i}^{i+1} G(n) d n \equiv \sum_{i=1}^{N} H_{i}
$$

where we have define $x_{N+1}=x_{1}$ and $y_{N+1}=y_{1}$. Note that the above expression is still exact . The approximation comes in the choice of a scheme for evaluating the integrals $H_{i}$, since $G(n)$ is only known at the integer values of $n$. If $G(n)$ is substituted with a polynomial $P^{(q)}(n)$ of degree $q^{\dagger}$, obtained via a Lagrange interpolation using $q+1$ values of $G$ at the nodes, one has the an approximation of order $q$ to $H_{i}$, namely

$$
\begin{aligned}
H_{i}^{(q)} & \equiv \int_{i}^{i+1} P_{i}^{(q)}(n) d n \\
& =\int_{1}^{2} P_{i}^{(q)}\left(n^{*}\right) d n^{*}
\end{aligned}
$$

† We limit $q$ to odd values here to maintain the symmetry about the interval between $i<n<i+1$. 
where the need for the change of variables to $n^{*} \equiv n-i+1$ will become apparent in a moment, and :

$$
P_{i}^{(q)}(n)=\sum_{k=0}^{q} G_{i k}^{(q)} L_{k}^{(q)}(n)
$$

where $G_{i k}^{(q)}$ is the value of $G$ at node $i+k-(q-1) / 2$, and $L_{k}^{(q)}(n)$ is the Lagrange polynomial of degree $q$ defined by:

$$
L_{k}^{(q)}(n)=\prod_{\substack{j=0 \\ j \neq k}}^{q} \frac{n-j}{k-j}
$$

and can be formally rewritten:

$$
L_{k}^{(q)}(n)=\sum_{m=0}^{q} \alpha_{k m} n^{m}
$$

and the $\alpha_{\mathrm{km}}$ 's are obtained by comparing equal powers of $n$ is (A.16) and (A.15)t. Now substitution of (A.16) and (A.14) into (A.13) allows for direct evaluation of the integral, and it follows that:

$$
\begin{aligned}
H_{i}^{(q)} & =\sum_{k=0}^{q} G_{i k}^{(q)} \int_{1}^{2} L_{k}^{(q)}\left(n^{*}\right) d n^{*} \\
& =\sum_{k=0}^{q} \beta_{k}^{(q)} G_{i k}^{(q)}
\end{aligned}
$$

where:

$$
\beta_{k}^{(q)} \equiv \sum_{m=0}^{q} \alpha_{k m}(m+1)^{-1}\left(2^{m+1}-1\right)
$$

Thus the change of variables from $n$ to $n^{*}$ in (A.13) makes the $\beta$ 's independent of $i$, so that they are only calculated once for all nodes, all moments and all contours. The final expression for the moment $M$ evaluated with a qth order scheme takes therefore the simple form:

† Note that the $\alpha_{k m}$ 's are the same for each internodal integral $H_{i}$, for each moment $J_{m n}$ and for each contour, so that they need only be calculated once. 


$$
M^{(q)}=\sum_{i=1}^{N} \sum_{k=0}^{q} \beta_{k}^{(q)} G_{i k}^{(q)}
$$

It can be shown that the choice $q=1$ is equivalent to using the trapezoidal rule to evaluate $H_{i}$, which is equivalent to fitting a straight line between $G(i)$ and $G(i+1)-$ i.e. a first order polynomial. In fact for $q=1$ it is easy to see from (A.10) that:

$$
M^{(1)}=\sum_{i=1}^{N} \frac{F(i+1)+F(1)}{2}\left(y_{i+1}-y_{i}\right)
$$

Formula (A.18) is however not accurate enough for our purposes. For $\mathrm{N}$ nodes equally spaced on a circle of radius 1 , the average value of dy is $4 / \mathrm{N}$; thus we can estimate that $M-M(1)=O\left(N^{-2}\right)$. Since we usually work with less than or approximately $O(100)$ nodes, and since we need to be able to detect logarithmic changes in moments down to $O\left(10^{-6}\right)$ we have used the next lowest order symmetric scheme, which corresponds to $q=3$.

At that order the function $G(n)$ in (A.11) is interpolated between $n=i$ and $n=i+1$ by a cubic polynomial using the values of $G(i-1), G(i), G(i+1)$ and $G(i+2)$. Recall that to evaluate $G(n)$ at $n=i$ we need to calculate $d y / d n$ at the nodes. Clearly, to maintain the same accuracy as the cubic Lagrange interpolation, we need for $\mathrm{dy} / \mathrm{dn}$ a "five- point formula" (see for instance Burden and Faires 1985) which is of order four. We have here chosen the symmetric one, which gives the value of the slope at $x_{0}$ of a function $f(x)$ in terms of the values of the function at the four points $x_{0}-2 h, x_{0}-h, x_{0}+h$ and $x_{0}+2 h$, namely:

$$
\frac{d f}{d x}\left(x_{0}\right)=\frac{1}{12 h}\left[f\left(x_{0}-2 h\right)-8 f\left(x_{0}-h\right)+8 f\left(x_{0}+h\right)-f\left(x_{0}+2 h\right)\right]+O\left(h^{4}\right)
$$

for $h<<1$. With the combination of a cubic Lagrange interpolant and and a five-point formula we have therefore built an algorithm that is accurate to $O\left(N^{-4}\right)$ which, for $\mathrm{N}=\mathrm{O}(100)$, has proven to be more than adequate for our purposes.

As a last check, we have actually verified numerically the order of this algorithm - as well as the the one of the scheme given in (A.18) - by means of a simple Richardson extrapolation performed with a ellipse off the origin and inclined at some non-zero angle with the $x$-axis. 


\section{Appendix B}

\section{Explicit Formulas for V-State Algorithms}

\section{B1: Rotating V-States $(\mathrm{m}=2)$}

For this type of $\mathrm{V}$-state the expressions anlogous to (1.10) for the angular velocity $\Omega$ and to (1.12) for the Fi's are identical to the ones for the Euler $V$-states given by $\mathrm{Wu}$ et al. (1984). Thus:

$$
\Omega=\frac{2}{R_{A}^{2}-R_{B}^{2}} \sum_{i=1}^{N}\left[u_{i+1 / 2}\left(x_{i+1}-x_{i+1}\right)-v_{i+1 / 2}\left(y_{i+1}-y_{i+1}\right)\right]
$$

and

$$
F_{i+1 / 2}=\frac{u_{1 / 2} \sin \theta_{i+1}-v_{i+1 / 2} \cos \theta_{i+1}+(\Omega / 2) R_{i+1}}{u_{i+1 / 2} \sin \theta_{i}-v_{i+1 / 2} \cos \theta_{i}+(\Omega / 2) R_{i}}
$$

We remark that we did not place nodes at equal polar angles for this type of V-state. Rather, since Zou et al. (1988) have shown that placing nodes such that their density is proportionally to the curvature to the power $1 / 3$ is optimal for the Kirchhoff ellipse, and since (as we have learnt a posteriori) the shapes of the $\mathrm{V}$ states are very close to ellipses, we decided to place the nodes at equal elliptical anglest, which, for an exact ellipse, is equivalent to their density going like $\kappa^{1 / 3}$.

T The elliptical angle $\eta$ is implicitly defined by the relations $x=a \cos \eta$ and $y=b \sin \eta$ for any point $(x, y)$ located on an ellispe of major axis a and minor axis $b$. 


\section{B2: Translating V-States}

Here again the formulas for $V$ and the $F_{i}$ 's are identical to the ones of Wu et al. (1984), namely:

$$
V=\frac{1}{x_{A}^{2}-x_{B}^{2}} \sum_{i=1}^{N}\left[u_{i+1 / 2}\left(x_{i+1}-x_{i+1}\right)-v_{i+1 / 2}\left(y_{i+1}-y_{i+1}\right)\right]
$$

and

$$
F_{i+1 / 2}=\frac{u_{1 / 2} \sin \theta_{i+1}-\left(v_{i+1 / 2}-V\right) \cos \theta_{i+1}}{u_{i+1 / 2} \sin \theta_{i}-\left(v_{i+1 / 2}-V\right) \cos \theta_{i}}
$$

Since they are a consequence of the topology of the vorticity field alone, these expressions apply equally to the case when the two vortices either are in the same or in different layers.

\section{B3: Doubly-Connected Rotating V-States}

The formulas for $\Omega$ and the $F_{i}$ 's applicable to this geometry are easily derived by generalizing Wu et al. (1984) and are found to be:

$$
\Omega=\frac{2}{x_{A}^{2}-x_{B}^{2}} \sum_{i=1}^{N}\left[u_{i+1 / 2}\left(x_{i+1}-x_{i+1}\right)-v_{i+1 / 2}\left(y_{i+1}-y_{i+1}\right)\right]
$$

and

$$
F_{i+1 / 2}=\frac{u_{1 / 2} \sin \theta_{i+1}-v_{i+1 / 2} \cos \theta_{i+1}+(\Omega / 2)\left(R_{i+1}+2 x_{0} \cos \theta_{i+1}\right)}{u_{i+1 / 2} \sin \theta_{i}-v_{i+1 / 2} \cos \theta_{i}+(\Omega / 2)\left(R_{i}+2 x_{0} \cos \theta_{i}\right)}
$$

where we have defined:

$$
x_{0}=\frac{x_{A}-x_{B}}{2}
$$

Since for this type of V-states we have been particularly interested in the limiting cases which have regions of very high curvature, the nodes were not placed at either polar or elliptical equal angles. Rather we have procedeed by increasing the density of nodes near either $x_{A}$ or $x_{B}$ or both according to the 
following simple procedure.

Since only the upper-right-hand quadrant need be considered (cf. Figure 2.18) focus only on the interval $0<\theta<\pi$, where $\theta$ is the polar angle measured from the point $\left(x_{0}, 0\right)$, the "center" of the right vortex. Now let $\theta_{i}$ be the value of $\theta$ for node $i$ and $i=1, N+1$ so that $\theta_{1}=0$ corresponds to $x_{B}$ and $\theta_{N+1}=\pi$ to $x_{A}$, and the contour is devided into $\mathrm{N}$ segments. Chosing a node placement scheme is then equivalent to giving an expression for $\theta_{i}$ as a function of $i$.

If the nodes are placed at equal angles it is elementary to see that the expression is simply:

$$
\theta_{i}=\pi\left\{\frac{i-1}{N}\right\}
$$

We have instead used the formula:

$$
\theta_{i}=\frac{\pi}{2}\left\{\cos \left[\pi\left(\frac{i-1}{N}\right)-\pi\right]+1\right\}
$$

which places in a smooth way more nodes near $\theta=0$ and $\pi$ and fewer nodes near $\theta=\pi / 2$. We have found that with (B3.2), not only is convergence greatly improved for the states with moderate curvature, but that, for the really spiky states, the algorithm will often converge to a spurious V-state when (B3.1) is used.

One may wonder whether, since the cusp usually develops either at $\theta=\pi$ or $\theta=0$, it wouldn't be even better to put more nodes only in the region were the curvature is high, and less everywhere else. In the same spirit as (B3.2) we have tried the formulas:

$$
\theta_{i}=\pi\left[\sin \left(\varphi_{i}-\frac{\pi}{2}\right)-1\right]
$$

and

$$
\theta_{i}=\pi \cos \left(\varphi_{i}-\frac{\pi}{2}\right)
$$

which put more nodes near $\theta=\pi$ and $\theta=0$, respectively, and where:

$$
\varphi_{i}=\frac{\pi}{2} \frac{i-1}{N}
$$




\section{9}

We have found that, in general, (B3.3) and (B3.4) perform less well than (B3.2) and we attribute this to the fact that the former produce such large differences in the internodal distances that the evaluation of the velocity as an integral over the contour is worsened sufficiently to produce a negative impact on the overall convergence.

Again, we recall that the above expressions hold for this type of $\mathrm{V}$-state independently of whether the vortices are in the same or in different layers. 


\section{Appendix C}

\section{Desingularized Two-Layer Contour Dynamics}

The exact expression for the velocity at a point $(x, y)$ in layer $i=1,2$ is given by equation (1.7):

$$
\left[u_{i}, v_{i}\right](x, y)=-\frac{1}{2 \pi} \sum_{j=1}^{2} \sum_{n=1}^{N j}\left\{\Pi_{j}(n)\right\} \int_{\partial D_{j}(n)} G_{i j}(r)[d \xi, d \eta]
$$

where $\mathrm{N}_{\mathrm{j}}$ is the number of closed regions of nonzero geostrophic potentyialvorticity in layer $\mathrm{j}$, and

$$
r=\left[(x-\xi)^{2}+(y-\eta)^{2}\right]^{1 / 2}
$$

The Greens' functions $G_{i j}$, given in (1.4), contain both logarithmic and modified Bessel $\left(K_{0}\right)$ terms which are singular at vanishing distances.

For the Euler equations the logarithmic singularity at the origin can be eliminated by means of a simple integration by parts (cf., for instance, Zou et al. 1987). Thus the Euler analog to (C.1), viz.

$$
[u, v](x, y)=\frac{1}{2 \pi p} \sum_{n=1}^{N}\left\{Q_{n}\right\} \int_{\partial D_{n}} \log \left(r^{p}\right)[d \xi, d \eta]
$$

where, usually, $\mathrm{p}=1$ or 2 , becomes, after integration by parts:

$$
[u, v](x, y)=\frac{1}{2 \pi p} \sum_{n=1}^{N}\left\{Q_{n}\right\} \int_{\partial D_{n}}[(x-\xi),(y-\eta)] r^{-p} d r^{p}
$$

The same operation can be performed on the modified Bessel term of order zero in the Greens' function. For instance, the equivalent barotropic velocities given by

$$
[u, v]_{\text {e.b. }}(x, y)=-\frac{1}{2 \pi} \sum_{n=1}^{N}\left\{\Pi_{n}\right\} \int_{\partial D_{n}} K_{0}(\gamma r)[d \xi, d \eta]
$$


can be rewritten in terms of the modified Bessel function of order 1, leading to the logarithmically desingularized expression

$$
[u, v]_{\text {e.b. }}(x, y)=\frac{1}{2 \pi} \sum_{n=1}^{N}\left\{\Pi_{n}\right\} \int_{\partial D_{n}} \gamma K_{1}(\gamma)[(x-\xi),(y-\eta)] d r
$$

The advantage of this expression, which, we recall is still exact, resides in the fact that near the origin the function $\mathrm{K}_{1}(\mathrm{z})$ behaves like $1 / z$. A simple integration by parts has thus removed the logarithmic singularity. Expression (C.5) can be discretized in the way shown by Zou et al. (1987) to obtain a second order algorithm. 


\section{Appendix D}

\section{Numerical Determination of the Critical Points of the Corotating Streamfunction for a Perturbed Kirchhoff Ellipse}

From a time sequence of contours $\left\{x_{i}(t), y_{i}(t) \mid i=1, N(t)\right\}$ at $t=t_{1}, t_{2}, t_{3}, \ldots$ (with $\left.t_{2}=t_{1}+\Delta t, t_{3}=t_{2}+\Delta t, \ldots\right)$ we proceed as follows to determine the equilibrium points of the corotating streamfunction.

First one needs to determine the instantaneous angular velocity $\Omega(t)$. To do this we fit the contours at $t_{1}$ and $t_{3}$ with ellipses from the values of the moments of vorticity, as explained in Appendix A. This yields the values $\varphi_{1}$ and $\varphi_{3}$ of the angles between the major axes of the fitted ellipses and the x-axis. The instantaneous value of $\Omega$ at $t_{2}$ is then calculated using the second order formula:

$$
\Omega\left(t_{2}\right) \approx \frac{\varphi\left(t_{3}\right)-\varphi\left(t_{1}\right)}{2 \Delta t}
$$

Since the corotating streamfunction $\psi_{c}$ is related to the inertial streamfunction $\psi$ by the simple relationship:

$$
\psi_{c}(x, y, t)=\psi(x, y, t)-(1 / 2) \Omega(t)\left(x^{2}+y^{2}\right)
$$

the corotating velocity field is easily obtained from the inertial one:

$$
u_{c}(x, y)=u(x, y)+\Omega y \quad \text { and } \quad v_{c}(x, y)=v(x, y)-\Omega x
$$

The equilibrium points of the corotating streamfunction, the centers and saddles of $\psi_{\mathrm{c}}(\mathrm{x}, \mathrm{y}, \mathrm{t})$, are the points where both $u_{\mathrm{c}}$ and $v_{\mathrm{c}}$ are identically zero. The coordinates $\left(x_{c}, y_{c}\right)$ of the equilibrium points must then satisfy:

$$
u\left(x_{c}, y_{c}, t\right)=-\Omega(t) y_{c} \quad \text { and } \quad v\left(x_{c}, y_{c}, t\right)=+\Omega(t) x_{c}
$$

Since $u\left(x_{c}, y_{c}\right)$ and $v\left(x_{c}, y_{c}\right)$ can be thought of as "very" nonlinear functions of $x_{c}$ and $y_{c},(D .3)$ is simply a nonlinear algebraic system of equations in two unknowns, and must be solved numerically. We have solved it using a nonlinear secant method due to Wolfe (1959), which is part of the IMSL routine package (Version 9) under 
the name ZSCNT. We have found this algorithm to converge extremely fast (say $O(10)$ iterations) provided the initial guess is sufficiently good. Note that this method yields the location of the centers as well as the saddles of the corotating streamfunction.

Using the Euler version of (1.8) to calculate the velocity fields, with single precision, $O(100)$ nodes per contour and sufficiently small $\Delta t$ (usually $<0.25$ ) we have found the above procedure to yield values for $x_{c}$ and $y_{c}$ to 3 decimal figures when tested against the exact positions known for an unperturbed Kirchhoff ellipse.

The same nonlinear secant method was used to solve (D.3) for a variety of $V$-states, but the value of $\Omega$ used was the one obtained from the iterative algorithms described in Section 1.5.1 and Appendix B, and no time stepping was required. 


\section{References}

Abramowitz, M. and Stegun, I. 1964 Handbook of Mathematical Functions, (National Bureau of Standards, Applied math Series - 55).

Aref, H. 1983 Integrable, chaotic and turbulent vortex motions in two-dimensional flows, Ann. Rev of Fluid Mech. , 15, 345-389

Babiano A., Basdevant C., Legras, B. and Sadourny, R. 1986 Vorticity and passive scalar dynamics in two-dimensional turbulence, J. Fluid Mech. , 83, 379397.

Basdevant, C., Legras, B., Sadourny, R., and Beland, M. 1981 A study of barotropic model flows: intermittency waves and predictability, J. Atmos. Sci., 38, 2305-2326.

Batchelor, G.K. 1967 An introduction to Fluid Dynamics (C.U.P.)

Berk, H. and Roberts, K.V. 1970 The water-bag model, in "Methods of Computational Physics" (B. Alder, S. Fernbach and M. Rotenberg, eds.), Vol. 9 (Academic Press)

Burbea, J. 1980 Vortex motions and their stability, Proc. Nonlinear Phenomena in Math. Sci. , Arlington 1980 (Academic Press).

Burbea, J. and Landau, M. 1982 The Kelvin waves of vortex dynamics and their stability, J. Comput. Phys. , 45, 127-156.

Burden, R.L. and Faires, J.D. 1985 Numerical Analysis (Prindle, Weber \& Schmidt).

Carnahan, B., Luther, H.A. and Wilkes, J.O. 1969 Applied numerical methods (John Wiley \& Sons)

Charney, J.G. 1971 Geostrophic Turbulence, J. Atmos. Sci. , 28, 1087-1095. 
Christiansen, J.P. and Zabusky, N.J. 1973 Instability, coalescence and fission of finite-area vortex structures, J. Fluid Mech. , 61, 219.

Couder, Y. and Basdevant, C. 1986 Experiments and numerical studies of vortex couples in teo-dimensional flows, J.Fluid Mech. , 173, 225-251.

Deem, G.S. and Zabusky, N.J. 1978a Stationary V-states: interactions, recurrence and breaking, Phys. Rev. Lett. , 40, 859.

Deem, G.S. and Zabusky, N.J.' 1978b Stationary V-states: interactions, recurrence and breaking, in: K. Lonngren and A. Scott, eds., Solitons in Action (Academic Press), 277-293

Dritschel, D. 1985 The stability and energetics of corotating uniform vortices, J. Fluid Mech. , 157, 95-134.

Dritschel, D. 1986 The nonlinear evolution of configurations of uniform vorticity, J.Fluid Mech. , 171, 157-182.

Dritschel, D. 1988 Contour surgery: A contour dynamics method for long-time behaviour of inviscid, two-dimensional rotational flow, to appear in J. Comput. Phys. , 77, in press.

Dritschel, D. 1988a The repeated fialmentation of vorticity interfaces, J. Fluid Mech., in press.

Dritschel, D. $1988 \mathrm{~b}$ On the stabilization of shear instability by adverse shear, preprint submitted to J. Fluid Mech.

Dritschel, D. $1988 \mathrm{c}$ The existence, stability and nonlinear evolution of isolated, twfold symmetric distributions of vorticity in an ideal, two-dimensional fluid, submitted to J. Fluid Mech.

Flierl, G.R., Larichev, V.D., McWilliams, J.C. and Reznik, G.M. 1980 The dynamics of baroclinic and barotropic solitary eddies, Dyn. Atmos. Ocean , 5, 1-41.

Flierl, G.R. 1987 Isolated eddy mdels in geophysics, Ann. Rev. Fluid Mech. , 19, 493-530.

Flierl, G.R. 1988 On the instability of geostrophic vortices, J. Fluid Mech.., in press. 
Fornberg, B. 1977 A numerical study of two-dimensional turbulence, J. Comput. Phys. , 25, 1-31.

Griffiths, R.W. and Hopfinger, E.J. 1986 Experiments with baroclinic vortex pairs in a rotating fluid. J. Fluid Mech. , 173, 501-518.

Griffiths, R.W. and Hopfinger, E.J. 1987 Coalescing of geostrophic vortices. J. Fluid Mech. , 178, 73-97.

Helfrich, K.R, and Send, U. 1988 Finite-amplitude evolution of two-layer geostrophic vortices, J. Fluid. Mech. , in press.

Herring, J.R. 1980 Statistical theory of quasigeostrophic turbulence, J. Atmos. Sci. , 37, 969-977.

Hogg, N.G. and Stommel, H.M. 1985a The heton, an elementary interaction between discrete baroclinic geostrophic vortices, and its implication concernig heat-flow, Proc. $R$. Soc. Lond. , A397, 1-20.

Hogg, N.G. and Stommel, H.M. 1985b Hetonic explosions: the breakup and spread of warm pools as explained by baroclinic point vortices, J. Atmos. Sci., 42, 1465-1476.

Holloway, G. 1986 Eddies, waves, circulation and mixing: statistical geofluid mechanics, Ann. Rev. Fluid Mech. , 18, 91-147.

Hua, B.L. and Haidvogel, D.B. 1986 Numerical simulations of the vertical structure of quasi-geostrophic turbulence, J. Atnos. Sci. , 43, 2923-2936.

Jacobs, P.A. and Pullin, D.I. 1895 Coalescence of stretching vortices, Phys. Fluids, 28, 1619.

Kelvin, Lord 1880 On the vibrations of a columnar vortex, Phil. Mag. ,10, 155-168.

Kida, S. 1982 Stabilizing effect of finite core on a Karman vortex street, J.Fluid Mech. , 122, 487.

Kraichnan, R.H. 1967 Inertial ranges in two-dimensional turbulence, Phys. Fluids, 10, 1417-1423. 
Kraichnan, R.H. and Montgomery, D. 1980 Two-dimensional turbulence, Rep. Prog. Phys. , 43, 547-619

Lamb, H. 1945 Hydrodynamics (Dover).

Landau, L.D. and Lifschitz, E.M. 1959 Fluid Mechanics (Addison-Wesley).

Legras, B., Santangelo, P. and Benzi, R. 1988 High resolution numerical experiments for forced two-dimensional turbulence, Europhys. Lett. , 5, 37-42.

Leith, C., 1984 Minimum Enstrophy Vortices, Phys. of Fluids , 27, 1388-1395.

Love, A.E.H. 1893 On the stability of certain vortex motions, Proc. London Math. Soc. , 35(1), 18.

McWilliams, J.C., Flierl, G.R., Larichev, V.D. and Reznik, G.M. 1981 Numerical studies of barotropic modons, Dyn. Atmos. Ocean , 5, 219-238.

McWilliams, J.C. and Zabusky, N.J 1982 Interactions of isolated vortices I. Modons colliding with modons, Geophys. Astrophys. Fluid Mech. , 19, 207-227.

McWilliams, J.C. 1984 The emergence of isolated coherent vortices in turbulent flow, J. Fluid. Mech. , 146, 21-43.

McWilliams, J.C. 1988 Statistical properties of decaying geostrophic turbulence, $J$. Fluid Mech. , in press.

Meacham, S.P., Polvani, L.M. and Flierl, G.R. 1988 M.I.T. Technical Report.

Meiron, D.I., Saffman, P.C. and Schatzman, J.C. 1984 The linear two-dimensional stability of inviscid vortexstrets for finite-core vortices, J. Fluid. Mech., $147,187$.

Melander, M.V., Styczek, A.S. and Zabusky, N.J. 1984 Elliptically desingularized vortex model for the two-dimensional Euler equations, Phys. Rev. Lett. , 53, $1222-1225$. 
Melander, M.V., Zabusky, N.J. and Styczek, A.S. 1986 A moment model for vortex interactions of the two-dimensional Euler equations. I. Computational validation of a Hamiltonian elliptical representation, J. Fluid Mech. , 146, 95-115.

Melander, M.V., McWilliams, J.C. and Zabusky, N.J. 1987a Axisymmetrization and vorticity gradient intensification of an isolated two-dimensional vortex through filamentation, J. Fluid Mech. , 178, 137-159.

Melander, M.V., Zabusky, N.J. and McWilliams 1987b Asymmetric vortex merger in two-dimensions: Which vortex is victorious?, Phys. Fluids, 30, 2610-2612.

Melander, M.V., Zabusky, N.J. and McWilliams, J.C. 1988 Symmetric vortex merger in two dimensions: Causes and conditions, to appear in J.Fluid. Mech.

Michalke, A and Timme, A. 1967 On the inviscid instability of certain twodimensional vortex-type flows, J. Fluid Mech. , 29, 647-666.

Overman, E.A. and Zabusky, N.J. 1982a Evolution and merger of isolated vortex structures, Phys. Fluids , 25, 1297-1305.

Overman, E.A. and Zabusky, N.J. 1982b Coaxial scattering of Euler equation translating V-states via contour dynamics, J. Fluid Mech. , 125, 187-202.

Pedlosky, J. 1970 Finite amplitude baroclinic waves, J. Atmos. Sci. , 27, 15-30.

Pedlosky, J. 1979 Geophysical Fluid Dynamics, chap. 6 (Springer-Verlag).

Pedlosky, J. 1985 The instablility of continuous heton clouds, J. Atmos. Sci. , 42, 1477-1486.

Phillips, N.A. 1954 Energy transformations and meridional circulations associated with simple baroclinic waves in a two-level, baroclnic model. Tellus 6, 273286.

Pierrehumbert, R.T. 1980 A family of steady translating vortex pairs with distributed vorticity, J. Fluid. Mech. , 99, 129-144. 
Polvani, L.M., and Carton, X.J. 1988 The tripole: a new coherent vortex structure of incompressible two-dimensional flows, submitted to Geophys. Astrophys. Fluid Mech.

Potter, D. 1976 Water-bag methods in magnetohydrodynamics, in "Methods of Computational Physics" (J. Killeen, ed.), Vol. 16, pp. 43-83 (Academic Press).

Pratt, L.J. and Stern, M.E. 1986 Dynamics of potential vorticity fronts and eddy detachment, J. Phys. Ocean. , 16, 1101-1120.

Press, W.H., Flannery, B.P., Teukolsky, S.A. and Vetterling, W.T. 1986 Numerical Recipes (Cambridge Univesity Press).

Rhines, P.B. 1979 Geostrophic Turbulence. Annual Review of Fluid Mechanics, vol. 11, pp.401-441.

Rhines, P.B. and Young, W.R. 1982 Homogenization of potential vorticity in planetary gyres, J. Fluid. Mech, 122, 347-367.

Roberts, K.V. and Christiansen, J.P. 1972 Topics in computational fluid mechanics, Comput. Phys. Comm. , 3, 14.

Sadourny, R. 1985 Quasi-geostrophic turbulence, an introduction. InTurbolenza e predicibilità nella Fluidodinamica Geofisica e la Dinamica del Clima. Scuola internazionale di fisica Enrico Fermi, LXXXVIII, pp133-158.

Saffman, P.G. and Szeto, R. 1980 Equilibrium shapes of a pair of equal uniform vortices, Phys. Fluids , 23, 171-185.

Salmon, R. 1980 Baroclinic Instability and Geostrophic Turbulence, Geophys. Astrophys. Fluid Dyn. , 10, 25-52.

Salmon, R. 1982 Geostrophic Turbulence. In Topics in Ocean Physics, Scuola internazionale di fisica Enrico Fermi, Varenna, Italy, pp.30-78.

Stern, M.E. and Pratt, L.J. 1985 Dynamics of vorticity fronts, J. Fluid Mech. ,161, 513-532. 
Wan, Y.H. and Pulvirenti, M. 1985 Nonlinear stability of a circular vortex, Commun. in Math. Phys. , 99, 435-450.

Wiggins, S. 1988 Stirred but not mixed, Nature, 333, 395-396.

Williams, G.P. and Wilson, R.J. 1988 The stability and genesis of Rossby vortices, J. Atmos. Sci. , 45, 207-241.

Wolfe, P. 1959 The Secant Method for Simultaneous Non-Linear Equations, Communications of the ACM, 2.

Wu, H.M., Overman, E.A. and Zabusky, N.Z. 1984 Steady state solutions of the Euler equations in two dimensions. Rotating and translating $\mathrm{V}$-states with limiting cases. I. Numerical algorithms and results, J. Comput. Phys. , 53, $42-71$.

Young, W.R. 1985 Some interactions between small numbers of baroclinic geostrophic vortices, Geophys. Astrophys. Fluid Mech. , 33, 35-61.

Zabusky, N.J. 1981a Computational synergetics and mathematical innovation, J. Comput. Phys. 43, 195-249.

Zabusky,, N.J. 1981b Recent developments in contour dynamics for the Euler equations, Ann. New York Acad. Sci. , 373, 160-170

Zabusky, N.J. and McWilliams, J.C. 1982 A modulated pont vortex model for geostrophic beta-plane dynamics, Phys. Fluids , 25, 2175-2182.

Zabusky, N.J. 1984 Computational synergetics, Phys. Today (July 1984) 36-45.

Zabusky, N.J. 1985 Computational Synergetics and the Exploration of Nonlinear Science, Lett. in Math. Phys. , 10, 143-147.

Zabusky, N.J. 1986 Visualizing mathematics: Evolution of vortical flows, Physica 18D, 15-25.

Zabusky, N.J., Hughes, M.H. and Roberts, K.V. 1979 Contour dynamics for the Euler equations in two dimensions, J. Comput. Phys. ,30, 96-106. 